\title{
Outlet Glacier and Landscape Evolution of Victoria Land, Antarctica
}

\author{
By Ross Whitmore
}

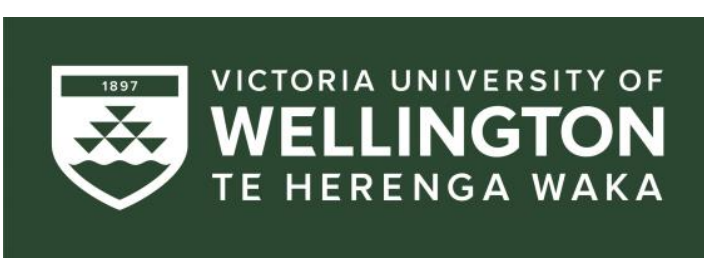

Antarctic Research Centre

Victoria University of Wellington

A Thesis submitted to Victoria University of Wellington In fulfilment of the requirements for the degree of

Doctor of Philosophy

In GEOLOGY

June 2020 



\section{Fire and Ice By Robert Frost}

Some say the world will end in fire, Some say in ice.

From what I've tasted of desire I hold with those who favor fire. But if it had to perish twice, I think I know enough of hate To say that for destruction ice Is also great And would suffice. 



\begin{abstract}
Terrestrial cosmogenic exposure studies are an established and rapidly evolving tool for landscapes in both polar and non-polar regions. This thesis takes a multifaceted approach to utilizing and enhancing terrestrial cosmogenic methods. The three main components of this work address method development, reconstructing surface-elevation-changes in two large Antarctic outlet glaciers, and evaluating bedrock erosion rates in Victoria Land, Antarctica. Each facet of this work is intended to enhance its respective field, as well as benefit the other sections of this thesis.
\end{abstract}

Quartz purification is a necessary and critical step to producing robust and reproducible results in terrestrial cosmogenic nuclide studies. Previous quartz purification work has centred on relatively coarse sample material $(1 \mathrm{~mm}-500 \mu \mathrm{m})$ and is effective down to $125 \mu \mathrm{m}$. However, sample material finer than that poses significant purification challenges and this material is usually discarded. The new purification procedure outlined in this thesis shows that very fine sand size material $(125-63 \mu \mathrm{m})$ can be reliably cleaned for use in terrestrial cosmogenic nuclide studies. The results below show that $35 \%$ mass loss in very fine-grained quartz is sufficient to remove major elements (Al, Ti, Na, K, Fe, Mg, Ca, Mn, ) and trace elements $\left({ }^{9} \mathrm{Be}\right.$, and ${ }^{10} \mathrm{~B}$ ) along with meteoric ${ }^{10} \mathrm{Be}$. Insufficient leaching is most detrimental to $\mathrm{Al}$ concentration, however errors up to $27 \%$ in exposure age and up to $29 \%$ in erosion rate are possible if meteoric ${ }^{10} \mathrm{Be}$ is not fully removed from quartz during the HF leaching stages.

Outlet glaciers have been well observed since the beginning of the satellite era, approximately 60 years ago. However, we do not currently know how these important glaciers, which drain a significant portion of the Antarctic Ice Sheet, have behaved on centennial to millennial timescales. Dating glacial erratics deposited by a thinning outlet glacier provides a window 
into the long-term outlet glacier and ice sheet response to climatic forcing. New results in this thesis constrain the thinning history of Mawson and Tucker glaciers over the last several thousand years. Mawson Glacier undergoes rapid thinning from at least $\sim 6.5$ kya to $\sim 4.9$ kya then transitions to slower thinning until $\sim 1 \mathrm{kya}$, with a minimum of $250 \mathrm{~m}$ of ice-surfacelowering. While Tucker Glacier $\sim 450 \mathrm{~km}$ north undergoes gradual thinning from $\sim 19$ kya to $\sim 5$ kya with $\sim 300 \mathrm{~m}$ of ice-surface-lowering. The results of this work show that either the Tucker Glacier was not significantly affected by the Ross Ice Shelf grounding line, or that Antarctic mountain glaciers respond differently to the outlet glaciers connected to the Easty Antarctic Ice Sheet. The style, rate, magnitude, and duration of thinning is unique to each outlet glacier, even with similar climate forcing. The results of this work shed light on the style and duration of outlet glacier thinning and retreat that is possible following a climate perturbation.

Antarctica's average bedrock erosion rate is consistently lower than $4.5 \mathrm{~m} / \mathrm{Myr}$, the lowest bedrock erosion rates for any region on Earth. Therefore, many cosmogenic dating studies assume zero erosion when calculating exposure ages. However, previous erosion rate work in Antarctica is biased to arid high-elevation inland sites ( $60 \%$ of work) and the hyperarid icefree McMurdo Dry Valleys ( $40 \%$ of work). These studies do not capture the effects of coastal maritime climates, where many outlet glacier studies are conducted, on the rate of bedrock erosion. New results presented in this thesis show that the Northern Victoria Land coast has the highest known erosion rate in Antarctica. Two sample sites were selected, one coastal and one in the interior. The coastal bedrock erosion rates are $8.86 \pm 0.78 \mathrm{~m} / \mathrm{Myr}$ and $7.15 \pm 0.6 \mathrm{~m} / \mathrm{Myr}$ while the interior bedrock erosion rates are $1.07 \pm 0.08 \mathrm{~m} / \mathrm{Myr}$ and $0.42 \pm 0.03 \mathrm{~m} / \mathrm{Myr}$. The coastal erosion rates are average for non-polar cold climates while the inland sites are below average for polar erosion rates. The results suggest a strong gradient in the rate of erosion is present from the Antarctic coastline inland. If exposure ages are not 
calculated with an appropriate erosion rate the apparent age may under-estimate the actual age by as much as $12 \%$, which is thousands of years for Holocene thinning histories like those found in this thesis. 



\section{ACKNOWLEDGEMENTS}

This work would not have been possible without a grant from the Ministry of Business Innovation and the Employment: Past Climates Program (E1704-541-1 to Mackintosh) and a grant from the Antarctic Science Bursary (E3287-3755 to Whitmore).

I could not have done this without the guidance of Andrew Mackintosh, Kevin Norton, and Cliff Atkins. The three of you indulged my broad range of interests and pushed me to be better. I need to thank my partner in crime Annika Taylor for always being better to me then I am to myself. A special thanks to Tracy Bean, Bia Broucinhas, James Hamilton, and Mike Rowe, for your work in the field while collecting rocks. A huge thanks to Heff, Jason, and Mark for the wonderful times we had flying around Antarctica. All of my friends: Karsten, Jamey, Weezy, The Jurganator, Marcelle, Claire, Cassandra, Shaun, Jenny, and all of the others I am glad to have met and laughed with you. Laurine and Hannah you always kept the office conversation lively... yeah, we will go with lively, at any rate I'm glad to know you. I need to recognize all of my previous mentors and the impact they have had on my life: John Spengler, Kathryn Bott, Janelle Adams, Cindy Hoack, Bill Nesse, Ken Hopkins, Ed Ripley, Bruce Douglas, Pat Cashman, Jim Trexler, Dan Sturmer, Tom Anderson, Dave Brown, Russell Shapiro, Candace Kairies-Beatty, and Lee Beatty. All of you have helped me reach goals I did not know I could.

I would be remiss if I did not thank some of my former students who taught me that you can still laugh and be kind even in really tough situations. I'm looking at you Bailer, Stephanie, Jack, Ameillia, Henry, and Hannah.

The following bands/composers were instrumental (no pun intended) in helping me through the hard days of graduate school the second time around: A Perfect Circle, Audioslave, Bad 
Religion, Children of Bodem, Corporate Avenger, Coal Chamber, Cold, Die So Fluid, Disturbed, Dry Kill Logic, Five Finger Death Punch, Godsmack, Garbage, Incubus, The Killers, Machine Head, Muse, Mudvayne, Motograter, Mushroomhead, Mozart, Marilyn Manson, Nick Cave and the Bad Seeds, Orgy, Pink Floyd, Plain White T's, Powerwolf, Queens of the Stone Age, Radiohead, The Red Hot Chili Peppers, Rise Against, Rob Zombie, Ray La Montaigne and the Pariah Dogs, Rammstein, Slipknot, Spineshank, System of a Down, Skinny Puppy, Snot, Tool, Vivaldi, White Zombie, Woodkid, Yo Yo Ma, and Zack Hemsey.

I would also like to thank the following authors for creating worlds that helped me escape my own: Franke Herbert, Andy Wier, James S.A. Corey, Kim Stanley Robinson, Herbert Phillip Lovecraft, Robert Heinlein, Arthur C. Clark, Isaac Asimov, and Margert Atwood.

Finally, I need to thank my parents, siblings, aunts, uncles, Jeff, Meeka-moo, Erica, and my in-laws for always believing in me. Even though I'm on the other side of the world, you are all in my thoughts; as I know I'm in yours. Thank you for all your loving support; I know I'm fortunate to have you all.

I will always cherish the time I spent on the ice and will share what I have seen as widely as possible. The time I spent in Antarctica has truly changed me, I love and appreciate my friends and family so much more now. In the $\sim 3$ months I spent on the ice I learned how forgiveness can liberate your spirit, and serious introspection can change your attitude. 


\section{TABLE OF CONTENTS}

Page

ABSTRACT

$\mathrm{V}$

LIST OF ABBREVIATIONS $\quad$ i

1 INTRODUCTION 3

1.1 Cosmogenic Dating: A tool for investigating landscape and ice sheet behaviour 3

1.1.1 Quartz Purification: Maximizing the potential of fine-grained samples for cosmogenic dating 3

1.1.2 Glacial thinning histories: Reconstructing outlet glaciers ice surface history 5

1.1.3 Erosion rates: Evaluating landscape evolution 6

2 BACKGROUND 9

2.1 Terrestrial cosmogenic nuclide background 9

2.1.1 Galactic Cosmic Radiation 9

2.1.2 Nuclide production through both neutron spallation and muon capture 12

$\begin{array}{lll}2.1 .3 & \text { Terrestrial cosmogenic nuclide decay } & 15\end{array}$

2.2 Quartz purification background 16

$\begin{array}{lll}\text { 2.2.1 Quartz purification procedures } & 17\end{array}$

$\begin{array}{ll}\text { 2.2.2 Context for work } & 18\end{array}$

2.3 Uses of terrestrial cosmogenic nuclides 20

2.3.1 Simple and complex exposure systems 20

2.3.2 Exposure dating 22

2.3.2.1 Calculations used to estimate exposure ages 22

2.3.3 Burial dating 24

2.3.3.1 Calculations used to estimate burial ages 25

2.3.4 Erosion rate 26

2.3.4.1 Calculations used to estimate erosion rates 27

2.3.5 Differential erosion 28

2.3.5.1 Calculations used to estimate differential erosion rates 29

2.3.6 Cosmogenic nuclide limitations 29

$2.4 \quad$ Ice sheet drainage pathways 32

2.4.1 Steady state ice flow 36

2.4.2 The marine ice sheet instability mechanism 40 
2.4.3 Meltwater pulse-1a

2.4.4 Ross Sea deglaciation history 45

2.5 Background on erosion rates 48

2.6 Research questions 50

3 METHODS $\quad 52$

3.1 General physical and chemical processing stages 52

3.2 Cosmogenic nuclide sampling strategies for ice-surface reconstructions 52

3.2.1 Sampling approach for age-elevation profiles 52

3.2.2 Glacial erratic sample selection criteria 55

3.3 Site selection and site assessment 55

3.3.1 Age-elevation transect site selection 56

3.3.2 Erosion rate site selection $\quad 58$

3.4 Calculating a linear thinning rate using IceTEA 58

4 QUARTZ PURIFICATION: QUANTIFYING THE BUCKET 60

$\begin{array}{lll}4.1 & \text { Introduction } & 60\end{array}$

$\begin{array}{lll}4.1 .1 & \text { Context } & 60\end{array}$

4.1.2 Aim of work $\quad 60$

4.2 Location and sample selection 60

$\begin{array}{lll}4.3 & \text { Method } & 60\end{array}$

$\begin{array}{lll}\text { 4.3.1 Sample description } & 61\end{array}$

4.3.2 Physical processing 63

$\begin{array}{lll}4.4 & \text { Experimental } & 64\end{array}$

4.4.1 Method design and digestion procedure $\quad 64$

$\begin{array}{lll}\text { 4.4.2 Initial setup-progress } & 65\end{array}$

4.4.3 Procedures for inductively coupled plasma mass spectrometer analysis $\quad 65$

4.4.4 Blank and standard scheme 68

4.4.5 Meteoric ${ }^{10} \mathrm{Be}$ removal 68

$\begin{array}{lll}4.5 & \text { Results } & 69\end{array}$

4.5.1 Total mass loss throughout the purification process 69

4.5.2 Fine-grained sample progression through chemistry 72

$\begin{array}{lll}\text { 4.5.3 Purification element data } & 73\end{array}$

4.5.3.1 Chemical series $1(10 \% \mathrm{HCl} \mathrm{X} \mathrm{2)} 76$ 
4.5.3.1 Chemical Series 2 and 3 (2.5\% HF and 1\% HF X 6) 79

4.5.3.2 Beryllium and Boron 82

4.5.3.3 Major Elements Removed by $\mathrm{HCl}(\mathrm{Fe}, \mathrm{Ca}, \mathrm{Mg}$, and $\mathrm{Mn}) \quad 83$

4.5.3.4 Major Elements removed by $\mathrm{HF}(\mathrm{Al}, \mathrm{Ti}, \mathrm{Na}$, and K) 84

4.5.4 Accelerator mass spectrometer data 84

4.5.4.1 Meteoric ${ }^{10} \mathrm{Be} \quad 84$

4.5.4.1 Digestion and Column Chemistry 89

4.6 Discussion of the new quartz purification procedure $\quad 91$

4.6.1 Ramifications of incomplete purification 91

4.6.2 Conservative quartz purification method comparison 92

4.6.3 The utility of very fine-grained quartz 93

5 MAWSON GLACIER: THINNING THROUGH THE HOLOCENE 95

$\begin{array}{lll}5.1 & \text { Introduction } & 95\end{array}$

$\begin{array}{lll}5.1 .1 & \text { Context } & 95\end{array}$

5.1.2 Aim of work 95

$\begin{array}{lll}5.2 & \text { Sampling } & 96\end{array}$

5.2.1 Sample collection 96

5.2.2 Bruce Point 99

5.2.3 Mount Murray 99

5.2.4 Accelerator mass spectrometry instruments 102

$\begin{array}{lll}5.3 \text { Results } & 102\end{array}$

$\begin{array}{lll}\text { 5.3.1 Lab blanks } & 102\end{array}$

$\begin{array}{lll}\text { 5.3.2 Mount Murray chronology } & 102\end{array}$

5.3.3 Holocene thinning of Mawson Glacier 105

$\begin{array}{lll}\text { 5.3.4 Sample outliers } & 109\end{array}$

5.3.5 Rate, timing and magnitude of thinning at Mawson Glacier 111

5.4 Discussion of Mawson Glacier's thinning history 111

5.4.1 Glacial History of the Southwestern Ross Sea 112

5.4.1.1 Timing and style of grounding line retreat from offshore records $\quad 114$

5.4.1.2 Correlating glacial records along the Scott Coast 115

5.4.1.3 Scott Coast history from local last glacial maximum 115

$\begin{array}{lll}\text { 5.4.1.4 Western Ross Sea bathymetry } & 119\end{array}$ 
5.4.1.5 Regional significance of thinning histories at Mawson and Tucker Glaciers 119

5.5 Evidence for a Mawson Glacier contribution to Meltwater pulse-1a 121

6 TUCKER GLACIER: THINNING SINCE THE LAST GLACIAL MAXIMUM 122

$\begin{array}{lll}6.1 & \text { Introduction } & 122\end{array}$

$\begin{array}{lll}\text { 6.1.1 Context } & 122\end{array}$

6.1.2 Aim of work 123

6.2 Sample collection and processing 123

6.2.1 Sample collection 123

6.2.2 Sample processing 126

6.2.3 Accelerator mass spectrometer analysis 126

$\begin{array}{lll}6.3 & \text { Results } & 126\end{array}$

6.3.1 Tucker Glacier chronology 126

6.3.2 Tucker Glacier thinning rate 129

6.3.3 Sample outliers 134

6.4 Discussion of Tucker Glacier's thinning history 134

6.4.1.1 Glacial history of the Northwestern Ross Sea 135

6.5 Evidence of a Tucker Glacier contribution to Meltwater pulse-1a 139

7 EROSION RATES IN VICTORIA LAND: LANDSCAPE EVOLUTION 141

$\begin{array}{lll}7.1 & \text { Introduction } & 141\end{array}$

$\begin{array}{lll}7.1 .1 & \text { Context } & 141\end{array}$

7.1.2 Aim of work 142

$\begin{array}{lll}7.2 & \text { Locations } & 142\end{array}$

7.2.1 West Trio Nunatak 144

$\begin{array}{lll}\text { 7.2.1.1 Sample collection } & 144\end{array}$

$\begin{array}{lll}\text { 7.2.1.2 Rock description } & 146\end{array}$

7.2.2 Bypass Ridge 146

$\begin{array}{lll}\text { 7.2.2.1 Sample collection } & 146\end{array}$

$\begin{array}{lll}\text { 7.2.2.2 Rock description } & 149\end{array}$

$\begin{array}{ll}\text { 7.2.3 Cape Christie } & 149\end{array}$

$\begin{array}{lll}\text { 7.2.3.1 Sample collection } & 151\end{array}$

$\begin{array}{lll}\text { 7.2.3.2 Rock description } & 151\end{array}$

7.3 Method and Theory 151 
7.4.1 Explanation of overall results

7.5 Discussion of new bedrock erosion rates from Victoria Land 159

7.5.1 Reanalysis and interpretation of bedrock erosion rates in Victoria Land 160

$\begin{array}{lll}\text { 7.5.2 Implications of assuming incorrect erosion rates } & 169\end{array}$

8 CONCLUSIONS 170

8.1 Quartz Purification Procedure 170

8.2 Mawson and Tucker Glaciers Thinning Histories 170

8.3 Bedrock Erosion Rates in Victoria Land 171

$8.4 \quad$ Impact of Results $\quad 172$

9 FUTURE WORK 174

9.1 Removal of Laurylamine from quartz samples 174

9.2 Ice surface reconstructions: Expanding the work presented 175

9.3 Erosion Rate Work: The need for further data 176

$\begin{array}{lll}9.4 & \text { Modelling of Tucker Glacier } & 177\end{array}$

10 Appendix A: Standard Physical Processing Methods $\quad 180$

$\begin{array}{lll}10.1 \text { Cutting } & 180\end{array}$

$\begin{array}{ll}\text { 10.1.1**Cleaning the Saw } & 180\end{array}$

$\begin{array}{ll}10.2 \text { Crushing } & 181\end{array}$

10.2.1**Cleaning the Rock Crusher 181

$\begin{array}{lll}10.3 & \text { Milling } & 182\end{array}$

10.3.1**Cleaning the Ring Mill 182

$\begin{array}{lll}10.4 & \text { Hand Magnetic Separation } & 183\end{array}$

$\begin{array}{lll}10.5 & \text { Frantzing } & 184\end{array}$

10.5.1**Cleaning the Frantz 184

11 Appendix B: Standard Chemical Processing Methods 186

11.1 Physical Quartz Separation 186

11.2 Chemical Quartz Cleaning and Etching 186

$\begin{array}{lll}11.3 & \text { Chemical Cleaning } & 188\end{array}$

11.3.1 Final Quartz Leach 188

11.3.2 Carrier addition and sample dissolution $\quad 189$

$\begin{array}{ll}\text { 11.3.3 Bulk dissolution (closed beaker) } & 189\end{array}$ 
11.3.4 Dissolution version 1: slow and steady (open beaker) 190

$\begin{array}{lll}11.4 & \text { Chemical Separation } & 192\end{array}$

11.4.1 Sample conversion and Al-chemistry 192

11.4.2 BeF2 - Leaching (only when NO Al-chemistry will be done) 193

11.4.3 Column $\mathrm{Fe} \quad 193$

11.4.4 5ml Column Be (Dirty Samples: Al > 5mg) 194

11.4.5 Column Al 195

$\begin{array}{ll}\text { 11.4.6 Be precipitation } & 196\end{array}$

11.4.7 Al precipitation 196

11.4.8 Be Oxide + Silver 197

$\begin{array}{ll}\text { 11.4.9 Targets } & 197\end{array}$

12 REFERENCES 200 


\section{LIST OF FIGURES}

\section{In Text Figures}

Figure 1.1 General Victoria Land and the Ross Sea map 8

Figure 2.1 Magnetic field cutoff rigidity 10

Figure 2.2 Secondary particle cascade 11

$\begin{array}{lll}\text { Figure 2.3 Spallation of }{ }^{16} \mathrm{O} & 13\end{array}$

Figure 2.4 Muon produced nuclides $\quad 15$

$\begin{array}{lll}\text { Figure 2.5 Mineral overgrowth removal } & 18\end{array}$

Figure 2.6 Simple versus complex exposure 21

Figure 2.7 Radiogenic decay of nuclides post burial 25

$\begin{array}{lll}\text { Figure } 2.8 \text { Differential nuclide decay } & 25\end{array}$

$\begin{array}{lll}\text { Figure 2.9 High erosion rate } & 27\end{array}$

Figure 2.10 Low erosion rates 27

Figure 2.11 Differential erosion 28

Figure 2.12 Old and young sample comparison 31

$\begin{array}{lll}\text { Figure 2.13 Outlet glaciers } & 34\end{array}$

Figure 2.14 A schematic diagram of an ice sheet 37

Figure 2.15 Cross section of a marine terminating glacier 40

Figure 2.16 Marine ice sheet instability 42

Figure 2.17 Grounded ice retreat hypotheses for the Ross Sea 47

Figure 2.18 Locations of published erosion rate studies 50

Figure 3.1 Shielding by glacial ice $\quad 53$

Figure 3.2 Glacier dipstick approach $\quad 54$

Figure 3.3 ArcSCENE image of Tucker Glacier 58

Figure 4.1 Hand sample in the field and in the lab 62

Figure 4.2 Thin section of sample HP-16-11 63

$\begin{array}{lll}\text { Figure } 4.3 & \text { Chemistry flowchart } & 64\end{array}$

$\begin{array}{lll}\text { Figure 4.4 Subsamples and supernates in solution } & 67\end{array}$

$\begin{array}{lll}\text { Figure 4.5 Timeseries of mass loss } & 71\end{array}$

$\begin{array}{lll}\text { Figure 4.6 Post } \mathrm{HCl} \text { photomicrograph } & 77\end{array}$

$\begin{array}{lll}\text { Figure } 4.7 & \text { Fe timeseries } & 77\end{array}$ 
$\begin{array}{lll}\text { Figure } 4.8 & \text { Ca timeseries } & 78\end{array}$

$\begin{array}{lll}\text { Figure } 4.9 & \text { Mn timeseries } & 78\end{array}$

$\begin{array}{lll}\text { Figure 4.10 } & \text { Mg timeseries } & 78\end{array}$

$\begin{array}{lll}\text { Figure 4.11 Post HF photomicrograph } & 80\end{array}$

$\begin{array}{lll}\text { Figure 4.12 Al timeseries } & 80\end{array}$

$\begin{array}{lll}\text { Figure } 4.13 \text { Ti timeseries } & 81\end{array}$

$\begin{array}{lll}\text { Figure 4.14 Na timeseries } & 81\end{array}$

$\begin{array}{lll}\text { Figure } 4.15 & \mathrm{~K} \text { timeseries } & 81\end{array}$

Figure 4.16 ${ }^{9} \mathrm{Be}$ timeseries $\quad 82$

$\begin{array}{lll}\text { Figure } 4.17 \text { B timeseries } & 82\end{array}$

Figure 4.18 Graph of normalised Be data 83

$\begin{array}{lll}\text { Figure } 4.19{ }^{10} \mathrm{Be} \text { purification timeseries } & 87\end{array}$

Figure 4.20 Blank corrected ${ }^{10} \mathrm{Be}$ atoms/gram semi-log plot $\quad 87$

$\begin{array}{lll}\text { Figure 4.21 Current strength timeseries } & 88\end{array}$

$\begin{array}{lll}\text { Figure 4.22 } & \text { Beam current and cation load } & 89\end{array}$

Figure 4.23 Pre- and post-treatment $\quad 90$

Figure 4.24 ${ }^{9} \mathrm{Be}$ current comparison graph 91

$\begin{array}{lll}\text { Figure 5.1 Mawson Glacier location map } & 97\end{array}$

$\begin{array}{lll}\text { Figure 5.2 Mawson Glacier ice velocity map } & 98\end{array}$

$\begin{array}{lll}\text { Figure 5.3 Mawson Glacier transect map } & 101\end{array}$

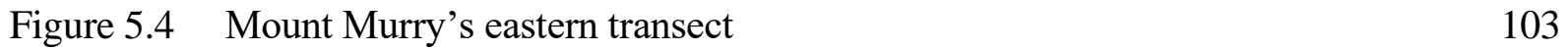

$\begin{array}{lll}\text { Figure 5.5 Mount Murray's western transects } & 104\end{array}$

$\begin{array}{lll}\text { Figure 5.6 Mawson age elevation plots } & 108\end{array}$

$\begin{array}{llr}\text { Figure 5.7 } & \text { Mawson and Mackay glaciers } & 113\end{array}$

Figure 5.8 Summary map of marine and terrestrial data from the Scott Coast and the McMurdo Sound 118

Figure 6.1 Heff's Manor and the Shark Fin sample locations 125

$\begin{array}{lll}\text { Figure 6.2 Heff's Manor sample locations } & 128\end{array}$

Figure 6.3 Heff's Manor chronology 132

Figure 6.4 Heff's Manor and Shark Fin chronology 132

Figure 6.5 Simplified Heff's Manor and Shark Fin chronology 133

Figure 6.6 Monte Carlo least squares linear regression for Heff's Manor and the Shark Fin133 
Figure 6.7 Bathymetric record offshore Tucker Glacier 136

$\begin{array}{lll}\text { Figure } 6.8 & \text { Tucker Glacier model } & 138\end{array}$

Figure 7.1 Sampling locations in Victoria Land: Antarctica 143

$\begin{array}{lll}\text { Figure 7.2 West Trio Nunatak } & 145\end{array}$

$\begin{array}{lll}\text { Figure } 7.3 & \text { Bypass Ridge } & 148\end{array}$

$\begin{array}{lll}\text { Figure 7.4 Cape Christie } & 150\end{array}$

Figure 7.5 Erosion rates in Victoria Land by elevation 162

Figure 7.6 Erosion rates in Victoria Land by proximity to coast (continentality) 162

Figure 7.7 Erosion rate in Victoria Land by latitude 163

$\begin{array}{lll}\text { Figure } 7.8 & \text { Victoria Land erosion rates } & 168\end{array}$ 



\section{LIST OF TABLES}

\section{In Text Tables}

Table 4.1 Timeseries of mass loss

$\begin{array}{lll}\text { Table } 4.2 \text { Acid supernate timeseries (ppm) } & 73\end{array}$

$\begin{array}{lll}\text { Table } 4.3 \text { Acid supernate timeseries }(\mu \mathrm{g}) & 74\end{array}$

Table 4.41 gram subsample timeseries (ppm) $\quad 75$

Table 4.5 Pre and post purification (ppm): $\quad 76$

Table 4.6 ${ }^{10}$ Be timeseries pre and post purification (ppm) 86

Table 4.7 Sample comparison $\quad 90$

Table 5.1 Sample and blank data: Mawson Glacier 106

Table 5.2 Mawson Glacier exposure ages 107

Table 5.3 Mount Murray outlier data 110

Table 6.1 Heff's Manor concentration calculations 130

Table 6.2 Heff's Manor age calculation and apparent exposure age 131

Table 7.1 Concentrations calculations 156

Table 7.2 Calculations for ages and erosion rates 156

Table 7.3 Apparent exposure age values 157

Table 7.4 Erosion rate values 157

Table 7.5 Calculated greywacke differential erosion rates 158

Table 7.6 Sample reanalysis of other Victoria land erosion rates 161

\section{APPENDICES}

Appendix A Standard Physical Processing Methods Used

Appendix B Standard Chemical Processing Methods Used 


\section{LIST OF ABBREVIATIONS}

\begin{tabular}{ll}
$\mathrm{km}$ & Kilometre \\
$\mathrm{m}$ & Metre \\
$\mathrm{m} / \mathrm{Myr}$ & Micrometre \\
$\mathrm{GeV}$ & Metres per Million Years \\
$\mathrm{asl}$ & Gigaelectron Volt \\
$\mathrm{p}^{+}$ & Above Sea Level \\
$\mathrm{n}^{0}$ & Proton \\
$\mathrm{n}_{\mathrm{th}}^{0}$ & Neutron \\
$\mathrm{e}^{-}$ & Thermal Neutron \\
$\mathrm{e}^{+}$ & Electron \\
$\mu^{-}$ & Positron \\
$\mu^{+}$ & Muon \\
$\alpha$ & Antimuon \\
$\pi^{-}$ & Alpha Particle \\
$\pi^{+}$ & Negatively Charged Pion \\
$\pi^{0}$ & Positively Charged Pion \\
$\kappa^{-}$ & Neutral Pion \\
$\kappa^{+}$ & Gamma Ray \\
$\kappa^{0}$ & Neutrino \\
\hline & Negatively Charged Kaon \\
\hline & Nositively Charged Kaon \\
\hline &
\end{tabular}




\section{INTRODUCTION}

\subsection{Cosmogenic Dating: A tool for investigating landscape and ice sheet behaviour}

In the last 30 years terrestrial cosmogenic nuclide applications have revolutionised the fields of geomorphology and glaciology. Measuring the quantity of one or more terrestrial cosmogenic nuclide provides the means to assess the exposure age of sediment or bedrock, determine the burial history for geological surfaces, and evaluate the rate of erosion for bedrock and watersheds. The different chemical and physical properties of the most widely used nuclides $\left({ }^{3} \mathrm{He},{ }^{10} \mathrm{Be},{ }^{14} \mathrm{C},{ }^{21} \mathrm{Ne},{ }^{26} \mathrm{Al}\right.$, and $\left.{ }^{36} \mathrm{Cl}\right)$ allow almost any rock type at any latitude and altitude to be evaluated with exposure ranging from hundreds to millions of years. This temporal range is ideal for investigating long-term geological and climatic trends. Directly constraining landscape and ice sheet histories provides a critical window into how the climate changes through glacial-interglacial cycles. The methods, and applications of this tool are explored in detail in this thesis and proceed from detailed to general topics, starting with quartz purification then transitioning to the implementation of both exposure dating and erosion rate assessment.

The overall aim of this work is to: 1) develop a method of purifying fine-grained quartz, 2) reconstruct the ice-surface-elevation-history of two outlet glaciers along the Victoria Land Coast, and 3) investigate how Antarctic erosion rates change over 5 degrees of latitude and from coastal conditions moving $50 \mathrm{~km}$ inland.

\subsubsection{Quartz Purification: Maximizing the potential of fine-grained samples for cosmogenic} dating

Terrestrial cosmogenic nuclides are formed in both the atmosphere and upper lithosphere by cosmic rays. These radiogenic and stable nuclides are used to evaluate a range of geological 
and glaciological questions. The evolution of this field relies on methodological advances that broaden the type and amount of material needed to successfully complete sample analysis. One of the most commonly used mineral/nuclide pairs used for quantitative geomorphology is quartz $/{ }^{10} \mathrm{Be}$. This system is widely used because ${ }^{10} \mathrm{Be}$ is produced by spallation of ${ }^{16} \mathrm{O}$, which is abundant in the crystal lattice, by a fast neutron $\left(\mathrm{n}^{0}\right)$. Additionally, quartz is stable at Earth's surface temperatures and pressures. The high concentration of oxygen in Earth's atmosphere means that ${ }^{10} \mathrm{Be}$ is also produced in the atmosphere at approximately 1000 time the rate it is produced in the lithosphere (Monaghan et al., 1985/86; Brown et al., 1991).

Sample purification is critically important for all in-situ terrestrial cosmogenic nuclide studies because it removes all meteorically-produced nuclides contained in mineral overgrowths and removes other elements which negatively impact the outcome of a terrestrial nuclide study. Specific contaminants may affect the elution of pure beryllium during column chemistry (Ochs and Ivy-Ochs, 1995) or interfere with the measurements made using an accelerator mass spectrometer (Corbett et al., 2016). The fundamental framework for mineral purification is found in two studies which loosely define the mass-loss and the reduction of meteoric ${ }^{10} \mathrm{Be}$ for monomineralic quartz with grain sizes between 710 and $500 \mu \mathrm{m}$ (Brown et al, 1991; Kohl and Nishiizumi, 1992). Subsequent work has expanded these purification procedures to explore other mineral/nuclide combinations (e.g. Pyroxene $/{ }^{10}$ Be (Blard et al., 2008; Eaves et al., 2018), carbonate $/{ }^{36} \mathrm{Cl}$ (Marrero et al, 2018), feldspar $/{ }^{10} \mathrm{Be}$ (Zerathe et al., 2017), quartz $/{ }^{14} \mathrm{C}$ (Goehring et al., 2014), and olivine $/{ }^{10} \mathrm{Be}$ (Caraceddo et al., 2018). In general, methodological advances such as these increase the ability of researchers to study new and different regions based on available rock types. 
I present a modified $\mathrm{HCl}$ and $\mathrm{HF}$ leaching method in this thesis. This work quantifies a quartz purification procedure for samples with a grain size between $125-63 \mu \mathrm{m}$ thereby establishing a framework to investigate rocks and sediment with very fine native grain size.

\subsubsection{Glacial thinning histories: Reconstructing outlet glaciers ice surface history}

The Amundsen Sea Sector of West Antarctica is currently experiencing mass loss (Jacobs et al., 2011; Favier et al., 2014; Arthern et al, 2017). Much of the West Antarctic Ice Sheet is grounded below sea level and may be vulnerable to unstable and irreversible grounding line retreat and rapid thinning (Weertman, 1974; Schoof, 2007ab; Church et al., 2013). By investigating areas which have experienced marine based deglaciation in the past we can better understand current ice sheet behaviour and use these critical observations to assess the current and future vulnerability of outlet glaciers. The western Ross Sea contains a record of the retreat history of marine terminating portions of East Antarctic outlet glaciers (Halberstadt et al., 2016; MaKay et al., 2016; Greenwood et al., 2017; Lee et al., 2017), which is ideal for reconstructing and assessing ice sheet instability processes (Figure 1.1).

Exposure ages from glacial erratics provide temporal data sets which can be used to understand the scale of changes experienced by the East Antarctic Ice Sheet over the last several thousand years (Anderson et al., 2014; Mackintosh et al., 2014). Crucially, terrestrial cosmogenic dating can reconstruct the ice surface history of an outlet glacier to illuminate the style, rate, magnitude, and timing of ice-surface-elevation changes (Jones et al., 2015; Spector et al., 2017; Anderson et al., 2017; Goehring et al. 2018, Balco et al., 2019). These paleo-records show when and how large ice sheets respond to changing climatic conditions throughout geological time. The style of deglaciation in this region shows that rapid thinning, when initiated, can progress unabated for hundreds of years (Jones et al., 2015; Spector et al., 2017). 
In this thesis I reconstruct the ice-surface-elevation histories for two outlet glaciers along the Victoria Land Coast. Mawson Glacier is an outlet glacier draining the East Antarctic Ice Sheet through the Transantarctic Mountain along the Scott Coast, while Tucker Glacier is a large alpine glacier independent of the East Antarctic Ice Sheet located in near Cape Adare along the Borchgrevink Coast (Figure 1.1).

\subsubsection{Erosion rates: Evaluating landscape evolution}

Erosion controls the production of sediment, long-term $\mathrm{CO}_{2}$ sequestration through chemical weathering, landscape evolution, the movement of mass from high to low elevation, and the production of soils (Berner et al., 1983; Hilley et al., 2008; Portenga et al., 2011). Erosion rates are typically used to quantify both basin-scale fluvial erosion and regional scale landscape evolution. Terrestrial cosmogenic nuclides provide critical insight to how these processes vary with different rock types, climatic regimes, and bedrock joint spacing among other variables (Portenga et ail., 2011; Marrero et al., 2018).

The consistently cold and hyperarid climate of Antarctica preserves landscapes and produces the lowest erosion rates on Earth (Portenga et al., 2011; Caraceddo et al., 2018, Marrero et al., 2018). Erosion rate studies also shed light on the long-term glacial history of Antarctica by identifying areas that were not overrun by ice for hundreds of thousands to millions of years (e.g. Brown et al., 1991; Brook et al., 1993; Oberholzer et al., 2003; Oberholzer et al., 2008; Di Nicola et al., 2012, Marrero et al., 2018). Erosion and weathering may be punctuated processes, dependent on specific climatic and ice sheet conditions (Caraceddo et al., 2018). Much of the work conducted in Antarctica has concentrated on areas inland from the coast and several have focussed specifically on the McMurdo Dry Valleys (Marrero et al., 2018); an atypical ice-free region. Expanding work outside of the McMurdo Dry Valleys can build a 
more complete understanding of the landscape evolution and glacial history of Antarctica (Figure 1.1).

New data presented in this thesis aims to explore erosion rates in Victoria Land along $\sim 5^{\circ}$ of latitude and from the coast up to $50 \mathrm{~km}$ inland. 


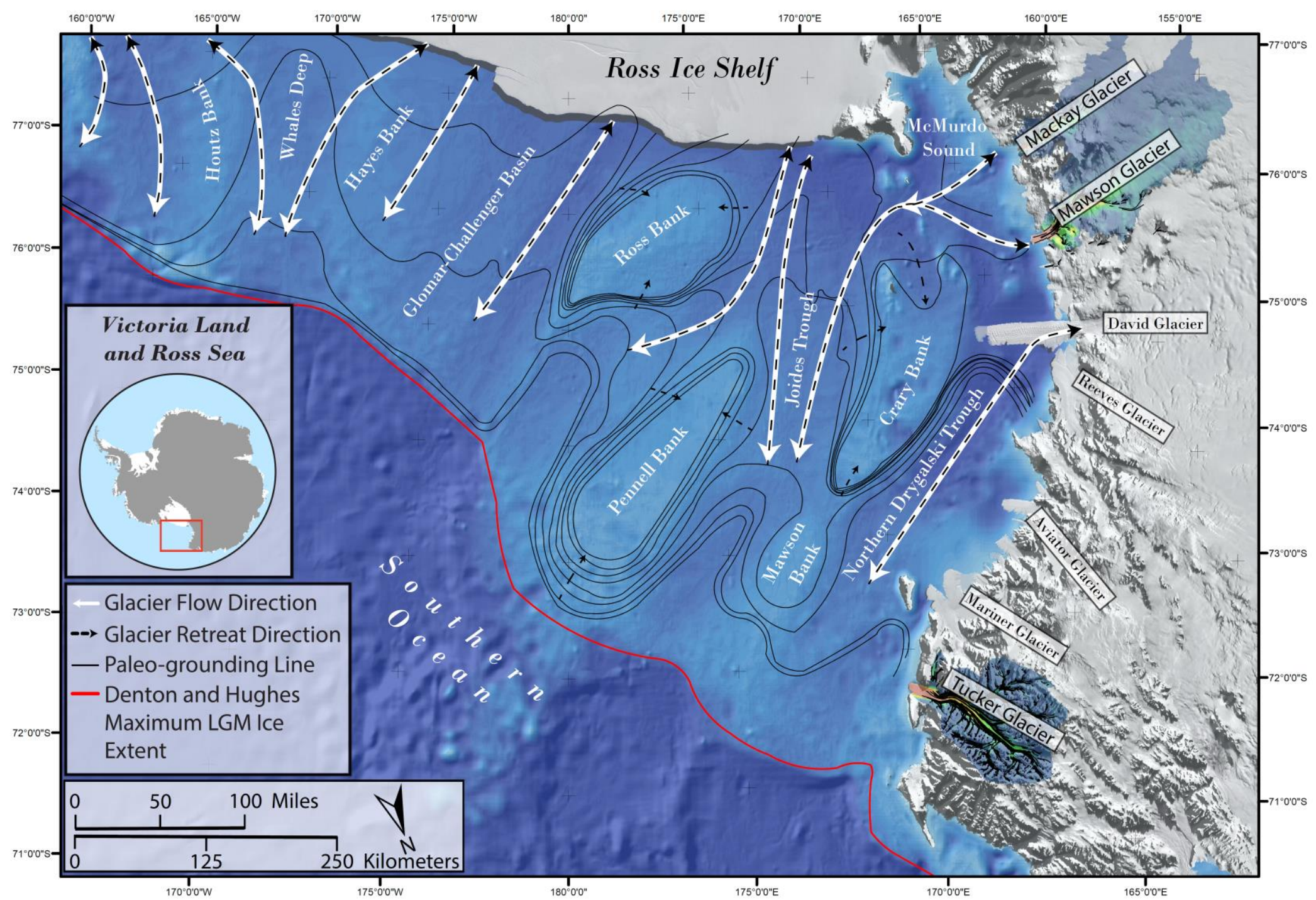

Figure 1.1 General Victoria Land and the Ross Sea map

This map shows the locations of places mentioned in the text as well the general orientation of megascale glacial lineation's (white arrows), grounding line retreat directions (black arrows), the marine based grounding line retreat model from Halberstradt and others (2017) (black lines), the hypothesized maximum extent of grounded ice during the Last Glacial Maximum of Denton and Hughes (2000) (red line 


\section{BACKGROUND}

The purpose of this section is to provide necessary and sufficient background information to each aspect of the specific projects undertaken and to identify research gaps and questions in the published scientific literature.

\subsection{Terrestrial cosmogenic nuclide background}

\section{$\underline{2.1 .1}$ Galactic Cosmic Radiation}

A cosmic ray is an energetic particle up to $10^{20}$ gigaelectron volts $(\mathrm{GeV})$ (Anderson, 1932; McKibben et al., 1982; Smart and Shea 1985). These particles can range in mass from protons $\left(\mathrm{p}^{+}\right)$and electrons (e $\mathrm{e}^{-}$) up to ionized atomic nuclei (Gaisser 1990). Approximately $80 \%$ of the total cosmic ray flux is from protons $\left(\mathrm{p}^{+}\right)$(Gosse and Phillips; 2001). These energetic particles are produced by galactic and extra-galactic sources, likely candidates include super nova and neutron stars (Smart, 1985; Gaisser, 1990). A division in the observed particle energy likely distinguishes between a Milky Way or an extra-galactic source; Particles with energies $<10^{18}$ $\mathrm{GeV}$ are thought to form within the Milky Way and particles with energies $>10^{18} \mathrm{GeV}$ are thought to be generated outside of the Milky Way (Gosse and Phillips; 2001). The more energetic the cosmic ray the more efficiently it penetrates the Earth's magnetic field (Figure 2.1). The interaction of cosmic rays with the Earth's magnetic field is complex but systematic. The magnetosphere forms a non-uniform barrier to energetic particles. The magnetic field has variable intensities and geometry from the equator to the poles (Shea et al., 1987; Smart and Shea, 2009; Pichowski et al., 2010; Herbst et al., 2013). Therefore, the cosmic ray flux is highest at high latitudes and lowest at low latitudes (Shea et al., 1987; Smart and Shea, 2009; Gosse and Phillips, 2001; Dunai, 2010; Balco, 2011) (Figure 2.1). Ray position, orientation, and intensity are unique to each cosmic ray and play a significant role in penetrating the 
magnetosphere and in the orientation of a secondary particle cascade relative to the ground surface (Greider, 2001). The ability of a cosmic ray to penetrate the magnetosphere is governed by the in-situ rigidity and the local geometry of the magnetic field at the point of intersection (Smart and Shea, 2009; Pichowski et al., 2010; Herbst et al., 2013) (Figure 2.1). The result is that near the northern and southern poles both high and low energy cosmic rays can penetrate the magnetic field, while at the equator only high-energy cosmic rays are able to do so (Figure 2.1) (Gosse and Phillips, 2001; Dunai, 2010).

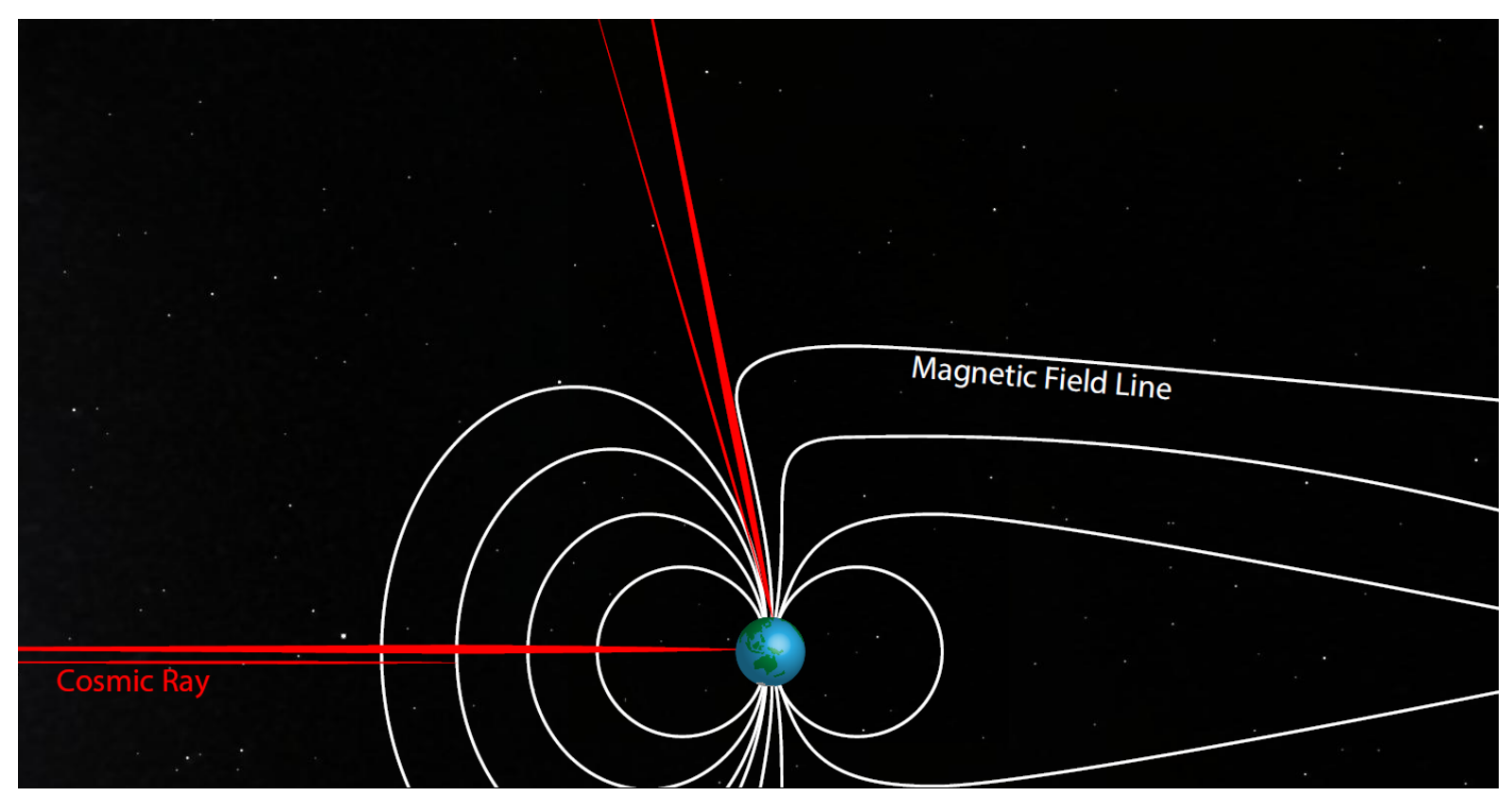

Figure 2.1 Magnetic field cutoff rigidity

The intensity and orientation of the Earth's magnetic field governs what cosmic ray energies are permitted to pass through the magnetosphere and interact with the Earth's atmosphere and lithosphere.

Cosmic rays which have penetrated the magnetosphere interact with the upper atmosphere and produce a secondary cascade of exotic particles (Figure 2.2). This particle cascade consists of a hadronic component (e.g. protons $\left(\mathrm{p}^{+}\right)$and neutrons $\left.\left(\mathrm{n}^{0}\right)\right)$, mesonic component (e.g. muons $\left(\mu^{-}\right)$and pions $\left.\left(\pi^{ \pm}\right)\right)$, and electromagnetic component (e.g. electrons $\left(\mathrm{e}^{-}\right)$and positrons $\left(\mathrm{e}^{+}\right)$) (Figure 2.2) (Gosse and Phillips; 2001). When these energetic particles interact with atoms in the atmosphere and lithosphere, they form cosmogenic nuclides through either spallation, neutron $\left(\mathrm{n}^{0}\right)$ capture, or muon $\left(\mu^{-}\right)$capture pathways (Gosse and Phillips, 2001). The inelastic 
behaviour of primary and secondary particles interactions gradually reduces the net particle energy (Figure 2.2). This energy reduction correlates with the depth of the atmosphere and final interaction with the lithosphere. Nuclides formed in the atmosphere are called meteoric cosmogenic nuclides whereas nuclides produced in the lithosphere are called in-situ terrestrial cosmogenic nuclide. Both the production of nuclides in the atmosphere and in the lithosphere can be exploited to better understand Earth's surface processes.

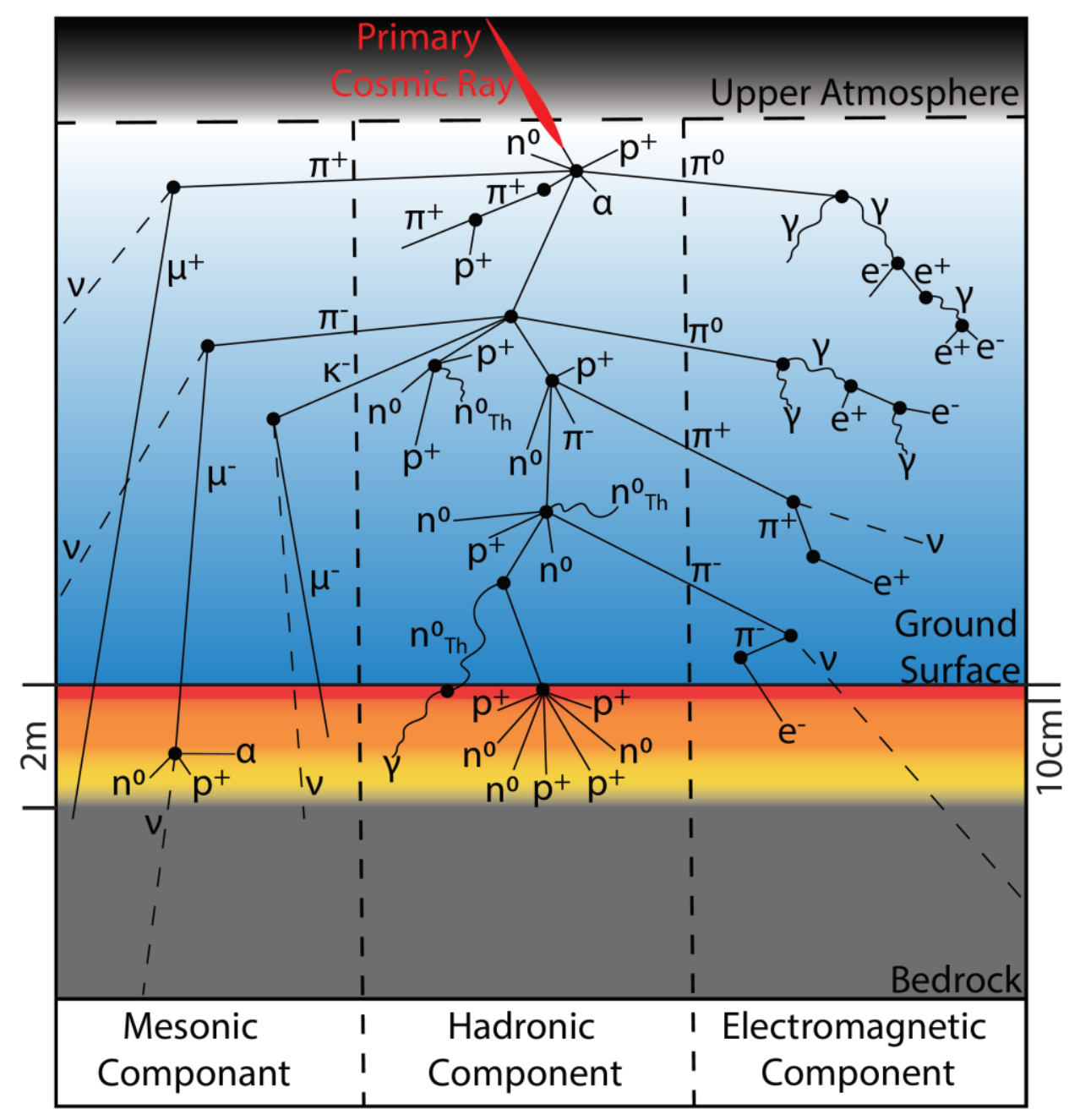

Figure 2.2 Secondary particle cascade

After a primary cosmic ray (red line) interacts with the Earth's upper atmosphere it produces a secondary cascade of energetic particles. The Hadronic Component (central column) is the critical production pathway for ${ }^{10} \mathrm{Be}$ and ${ }^{26} \mathrm{Al}$ through a process of spallation (see text for further discussion of spallation). The Mesonic Component is also capable of producing ${ }^{10} \mathrm{Be}$ through a process of muon $\left(\mu^{-}\right)$capture which is associated with a deeper production range. The maximum terrestrial cosmogenic nuclide production zone is the upper $10 \mathrm{~cm}$ of the Earth's surface (dark red line at the ground surface). The spallation production zone extends down to $\sim 2 \mathrm{~m}$ depth (red to yellow shaded zone, below the ground surface) and the muon $\left(\mu^{-}\right)$production zone extends down to 30m depth (not shown). Figure after Gosse and Phillips, 2001 and Greider, 2001. 
Cosmic ray orientations are unique to each incoming ray. Approximately $80 \%$ of the incoming cosmic ray flux is oriented at angles greater than $45^{\circ}$ from the horizon. Critically, the remaining $\sim 20 \%$ of cosmic ray flux can be blocked by near-field and far-field topography (Gosse and Phillips; 2001). Surrounding topography intercepts low-angle cosmic rays and reduces the oblique flux to a sample. Uncorrected heavily shielded samples yield a younger apparent exposure age than the true exposure age (Dunne et al. 1999; Benson et al., 2004; and Schildgen et al. 2005). To correct for topographic shielding, the inclination and declination of topography is measured when collecting samples.

\subsubsection{Nuclide production through both neutron spallation and muon capture}

Ninety eight percent of in-situ terrestrial cosmogenic nuclides are produced in the upper $2 \mathrm{~m}$ of the Earth's surface with the highest accumulation occurring in the top $10 \mathrm{~cm}$ of the production zone (Gosse and Phillips, 2001; Dunai, 2010; Dunai and Lifton 2014) (Figure 2.2). Both radiogenic (isotopes capable of radioactive decay) $\left({ }^{10} \mathrm{Be},{ }^{14} \mathrm{C},{ }^{26} \mathrm{Al},{ }^{36} \mathrm{Cl}\right.$, and ${ }^{53} \mathrm{Mn}$ ) and stable $\left({ }^{3} \mathrm{He}\right.$ and $\left.{ }^{21} \mathrm{Ne}\right)$ nuclides are produced in the atmosphere and/or lithosphere. Each stable and radiogenic nuclide is produced at predictable rates, and the radiogenic nuclides decay at known rates (Nishiizumi et al. 1989; Lal, 1991). Most nuclides are retained in the crystal lattice of the mineral in which it is produced (Smart and Shea, 1985, Gosse and Phillips, 2001); however, some nuclides have a high rate of diffusion in mineral they are produced in, such as ${ }^{3} \mathrm{He}$ in quartz (Trull et al., 1995; Schuster et al., 2005; Tremblay et al.,2014; Van Orman et al., 2015). Critically the half-life of the radiogenic isotope is different for each nuclide and can be exploited to determine the duration that a surface or sediment has been buried. The most commonly utilized nuclide series are ${ }^{10} \mathrm{Be}$ and ${ }^{26} \mathrm{Al}$. The half-life for ${ }^{10} \mathrm{Be}$ and ${ }^{26} \mathrm{Al}$ are $1.39 \mathrm{Ma}$ (Chmeleff et al., 2010; Korshinek et al., 2010) and 0.708 Ma (Nishiizumi, 2004), respectively. 
There are several minor hadronic production pathways for ${ }^{10} \mathrm{Be}(\mathrm{Mg}, \mathrm{Al}, \mathrm{Si}, \mathrm{Ca}$ (Masarik, 2002; Korber et al.,2005; Braucher et al, 2005)) and ${ }^{26} \mathrm{Al}(\mathrm{P}, \mathrm{S}, \mathrm{Cl}, \mathrm{K}$, and $\mathrm{Ca}$ (Skelton and Kavanagh 1987; Masarik, 2002)). Here the primary production pathway for ${ }^{10} \mathrm{Be}\left({ }^{16} \mathrm{O}+\mathrm{n}^{0}\right.$

$\left.{ }^{10} \mathrm{Be}+3 \mathrm{n}^{0}+4 \mathrm{P}^{+}\right)($Figure 2.3) is used to illustrate how spallation reactions produce cosmogenic nuclides. This process is initiated by a fast neutron $\left(\mathrm{n}^{0}\right)$ hitting the atomic nucleus of a ${ }^{16} \mathrm{O}$ atom. The ${ }^{16} \mathrm{O}$ nucleus shatters and ejects four protons $\left(\mathrm{p}^{+}\right)$and three neutrons $\left(\mathrm{n}^{0}\right)$ while leaving the nucleus of a new ${ }^{10} \mathrm{Be}$ atom (Figure 2.3) (Dunai and Lifton, 2014). The process is the same for ${ }^{26} \mathrm{Al}$, but the most common target element is ${ }^{28} \mathrm{Si}$ which exchanges an energetic neutron $\left(\mathrm{n}^{0}\right)$ for a proton $\left(\mathrm{p}^{+}\right)$in the atomic nucleus (Masarik, 2002). The combination of ${ }^{10} \mathrm{Be}$ and ${ }^{26} \mathrm{Al}$ is commonly used to date surface materials and explore surface processes because of the abundance and stability of quartz, which is composed primarily of ${ }^{28} \mathrm{Si}$ and ${ }^{16} \mathrm{O}$, at Earth's surface.

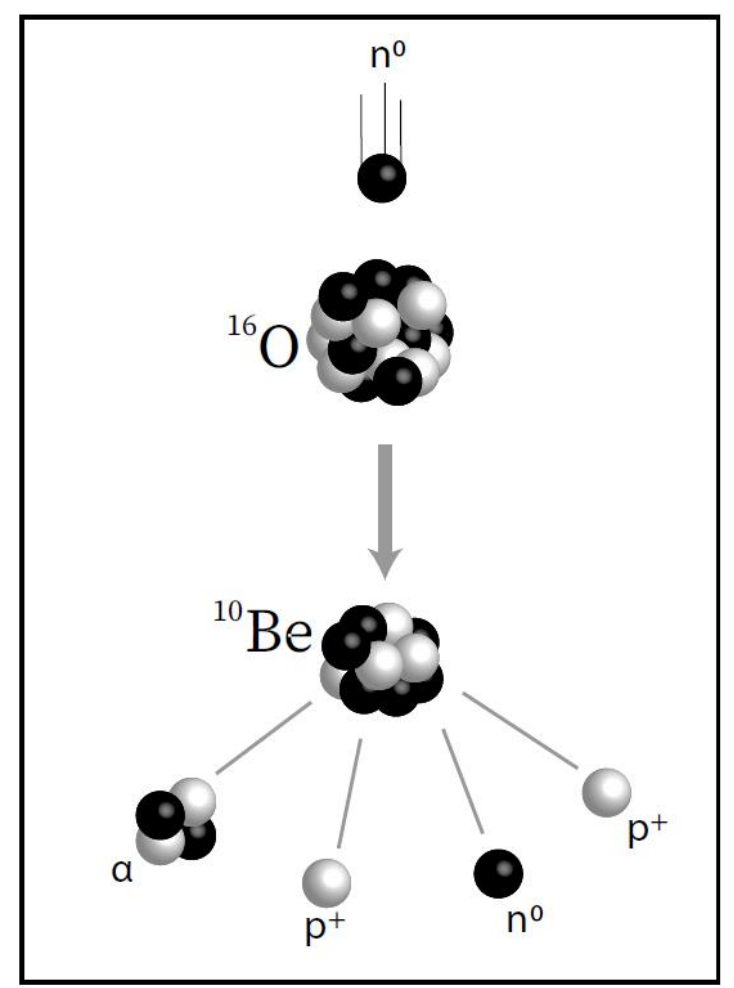

Figure 2.3 Spallation of ${ }^{16} \mathrm{O}$

The spallation reaction proceeds from the top to the bottom of the figure. The primary ${ }^{10} \mathrm{Be}$ production pathway is from a fast neutron $\left(\mathrm{n}^{0}\right)$ (black sphere labelled $\mathrm{n}^{0}$ ) shattering an ${ }^{16} \mathrm{O}$ atomic nucleus. When this interaction is done, several hadrons have been ejected from the atomic nucleus leaving the ${ }^{10} \mathrm{Be}$ nucleus behind. 
The remaining $2 \%$ of nuclide production is from muons $\left(\mu^{-}\right)$, which are part of the mesonic component of the secondary cascade. Critically, this particle is the only production pathway to make ${ }^{10} \mathrm{Be}$ and other nuclides at depth. A muon $\left(\mu^{-}\right)$is a small weakly-interacting charged particle produced by the decay of both pions $\left(\pi^{ \pm}\right)$and kaons $\left(\mathrm{K}^{ \pm}\right)$in the atmosphere $\sim 16 \mathrm{~km}$ above the Earth's surface (Figure 2.2) (Grieder, 2001; Gosse and Phillips, 2001; Dunai, 2010; Dunai and Lifton 2014). Due to the weak interaction of muons $\left(\mu^{-}\right)$with the surrounding environment effective nuclide production reaches a depth of $\sim 30 \mathrm{~m}$ in the ground (Dunai and Lifton, 2014). Two production pathways exist for muon $\left(\mu^{-}\right)$produced terrestrial cosmogenic nuclides: 1) "stopped" muon ( $\mu^{-}$st $)$production (Figure 2.4a) and 2) "fast" muon $\left(\mu_{\mathrm{F}}^{-}\right)$ production (Figure 2.4b) (Dunai and Lifton, 2014).

"Stopped" muons $\left(\mu^{-}\right.$st $)$are particles which were slowed by the interaction with other ionized material. When the muon is slow enough it is absorbed by the nucleus of a nearby atom. If this is an ${ }^{16} \mathrm{O}$ atom, the muon $\left(\mu^{-}\right)$will rapidly descend through the electron $\left(\mathrm{e}^{-}\right)$cloud to the nucleus where it neutralizes a proton $\left(\mathrm{p}^{+}\right)$. This neutralization reaction causes an alpha $(\alpha)$ decay, proton $\left(\mathrm{p}^{+}\right)$emission, neutron $\left(\mathrm{n}^{0}\right)$ emission, production of a neutrino $(\mathrm{v})$, and $\mathrm{a}^{10} \mathrm{Be}$ nucleus (Figure 2.4A).

Nuclide production by a "fast" muon $\left(\mu_{\mathrm{F}}^{-}\right)$is more complex, requiring several particle and photon interactions. Initially the "fast" muon $\left(\mu_{\mathrm{F}}^{-}\right)$is deflected by the electrical field of an atomic nucleus. This interaction produces a gamma ray photon $(\gamma)$, known as breaking radiation (Bremstrahlung). This gamma ray photon $(\gamma)$ can be energetic enough to produce a secondary neutron $\left(\mathrm{n}^{0}\right)$ from a different nucleus, which may produce ${ }^{10} \mathrm{Be}$ or other nuclides in a third atom (Figure 2.4B). Fast muons $\left(\mu^{-}\right)$are the only production mechanism below $20 \mathrm{~m}$ in the ground. 

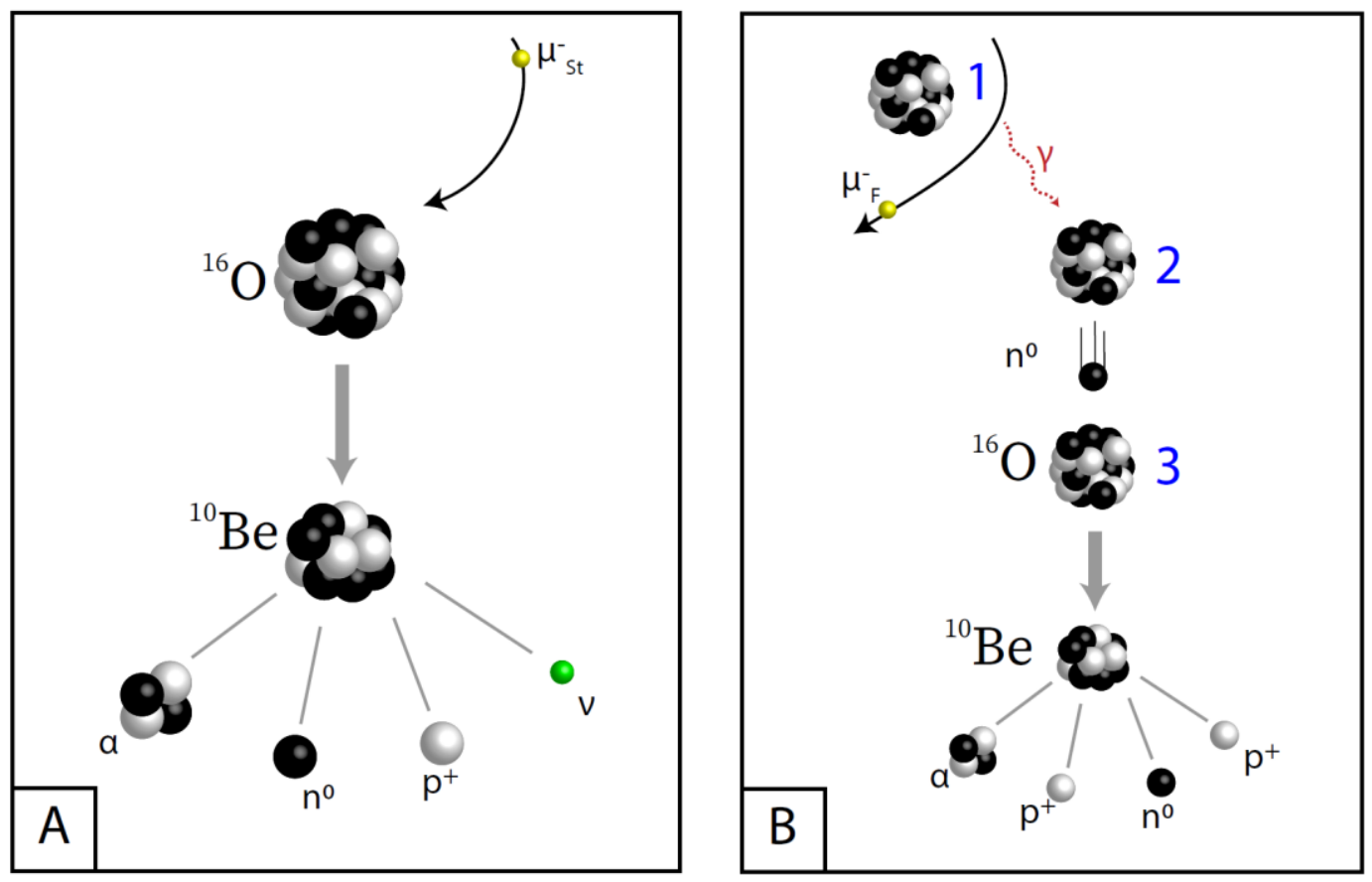

Figure 2.4 Muon produced nuclides

Both systems above produce ${ }^{10} \mathrm{Be}$ and other cosmogenic nuclides up to $30 \mathrm{~m}$ in the ground. A) Shows a stopped muon $\left(\mu^{-} \mathrm{st}\right)$ being incorporated into the nucleus of an ${ }^{16} \mathrm{O}$ atom. After the muon $\left(\mu^{-}\right)$has descended through the electron $\left(\mathrm{e}^{-}\right)$ shells it neutralizes a proton $\left(\mathrm{p}^{+}\right)$which destabilizes the atom. The atom emits a neutron $\left(\mathrm{n}^{0}\right)$, proton $\left(\mathrm{p}^{+}\right)$, alpha $(\alpha)$ particle, a neutrino $(v)$ and leaves a ${ }^{10}$ Be nucleus. B) Shows a fast muon $\left(\mu_{\mathrm{F}}^{-}\right)$interacting with the electric field of atom 1 and produce a gamma ray photon $(\gamma)$. If the photon $\left(\mathrm{p}^{+}\right)$is energetic enough it will produce a neutron $\left(\mathrm{n}^{0}\right)$ when it hits atom 2 . If the newly produced free neutron $\left(\mathrm{n}^{0}\right)$ is energetic enough it may produce a spallation reaction in atom 3.

\section{$\underline{2.1 .3}$ Terrestrial cosmogenic nuclide decay}

The half-life of both ${ }^{10} \mathrm{Be}$ and ${ }^{26} \mathrm{Al}$ are as important as their respective production rates. The half-life of ${ }^{10} \mathrm{Be}$ was recently revised to $1.39 \mathrm{my} \pm 0.013$ my (Chmeleff et al., 2010; Korshinek et al., 2010) and the half-life of ${ }^{26} \mathrm{Al}$ is now thought to be 0.708 my \pm 0.017 my (Nishiizumi, 2004). However, the previous decay rate values are still abundant in the literature (Thomas et al., 1984; Hofmann et al., 1987; Nishiizumi et al., 2000; Nishiizumi et al., 2007). Additionally, the type of radiogenic decay determines the daughter product; ${ }^{10} \mathrm{Be}$ decays through beta radiation into ${ }^{10} \mathrm{~B}$ while ${ }^{26} \mathrm{Al}$ decays through beta radiation to ${ }^{26} \mathrm{Mg}$. Both of these daughter products can be discriminated by gas filled detectors even though they are isobarically identical. The daughter products are orders of magnitude more abundant and stable then their parent nuclide, therefore, it necessary to remove as much daughter product (e.g. ${ }^{10} \mathrm{~B}$ and ${ }^{26} \mathrm{Mg}$ ) 
as possible from the sample. Effective removal or significant reduction of each of these decay products is needed before a sample can be analysed on an accelerator mass spectrometer.

\subsection{Quartz purification background}

This section is not intended as a thorough review of all aspects of the production of terrestrial cosmogenic nuclides but as a concise description of major sources of natural sample contamination. This is intended only to provide context for the importance of proper quartz purification and the role of major element, ${ }^{10} \mathrm{~B},{ }^{9} \mathrm{Be}$ and meteoric ${ }^{10} \mathrm{Be}$ contamination. For thorough reviews of all aspects of terrestrial cosmogenic nuclides processes see Gosse and Phillips (2001), Balco (2008), and Dunai (2010).

The three critical points of discussion for quartz purification are described below. 1) Cosmic rays are high-energy extra-solar particles which interact with Earth's atmosphere and lithosphere to produce cosmogenic nuclides. For ${ }^{10} \mathrm{Be}$ specifically the main parent element is ${ }^{16} \mathrm{O}$. This target atom is beneficial and problematic for terrestrial cosmogenic studies, because it is prevalent in both the mineral quartz and Earth's atmosphere. When an energetic neutron $\left(\mathrm{n}^{0}\right)$ interacts with an ${ }^{16} \mathrm{O}$ atom in either the atmosphere or in a silicate mineral, like quartz, it can produce ${ }^{10} \mathrm{Be}$ along with other nuclides (e.g. ${ }^{3} \mathrm{He}$ ). The meteoric component (produced in the atmosphere) and the terrestrial component (produced in the lithosphere) are indistinguishable from on another to the detector of an accelerator mass spectrometer.

2) When meteorically derived nuclides interact with rock surfaces they can adsorb to mineral overgrowths. For ${ }^{10} \mathrm{Be}$ several different oxides and quartz overgrowths are formed (Monaghan et al., 1985/86; Brown et al., 1991). These mineral overgrowths need to be removed by acid leaching. Importantly, other major elements and ${ }^{10} \mathrm{~B}$ can be incorporated in these overgrowths as well thus, further contaminating the rock sample. 
3) If samples coated with meteorically derived material and increased major elements are not effectively purified, they may fail in chemistry (due to the interfering elements during ion exchange) or produce anomalously old ages (due to excess meteoric ${ }^{10} \mathrm{Be}$ ). The main major elements of concern are $\mathrm{Al}, \mathrm{Ti}, \mathrm{Ca}, \mathrm{Mg}, \mathrm{B}$, and $\mathrm{Na}$. Aluminium and titanium are known to overload ion exchange resins during column chemistry (Corbett et al., 2016). Titanium in particular can dramatically reduce accelerator mass spectrometer beam current strength (Corbett et al., 2016), Ca can coelute with $\mathrm{Al}$ during column chemistry and complicate ${ }^{26} \mathrm{Al}$ studies (Ochs and Ivy-Ochs, 1997), and excess Na can elute with Be during column chemistry if acids are mixed incorrectly and potentially complicate $\mathrm{BeOH}$ precipitation. Magnesium is a problem because it is the next cation to elute after $\mathrm{Be}$ and tends to overlap with the Be. This is an issue because either some $\mathrm{Be}$ is forfeit before the elution is complete or some $\mathrm{Mg}$ is eluted and contaminates the Be sample. It is critical to thoroughly purify samples because ${ }^{10} \mathrm{~B}$ and ${ }^{26} \mathrm{Mg}$ are isobarically indistinguishable from cosmogenic ${ }^{10} \mathrm{Be}$ and ${ }^{26} \mathrm{Al}$ to an accelerator mass spectrometer and may result in low beam current which returns poor results.

\subsubsection{Quartz purification procedures}

After monomineralic quartz is obtained from physical processing, a series of acid leaching steps are used to remove major elements, meteoric ${ }^{10} \mathrm{Be}$ and any other natural contamination present. The acids commonly used are $\mathrm{HCl}, \mathrm{HF}$, and $\mathrm{HNO}_{3}$. The acid concentrations are kept low to preserve as much of the primary quartz core as possible while simultaneously removing as much of any mineral overgrowth as possible. Leaching phases progressively remove mineral overgrowths on grains, reduce major element contamination, and remove meteoric ${ }^{10} \mathrm{Be}$ from the sample (Brown et al. 1991) (Figure 2.5). 
The most significant material to be removed is meteoric ${ }^{10} \mathrm{Be}$ because it is 1,000 times more abundant in the environment then in-situ ${ }^{10} \mathrm{Be}$ and it readily adsorbs to quartz crystals (Monaghan et al., 1985/86; Brown et al. 1991). If meteoric ${ }^{10} \mathrm{Be}$ is present on mineral grains during digestion it is incorporated with the in-situ ${ }^{10} \mathrm{Be}$ and measured on the accelerator mass spectrometer as in-situ ${ }^{10} \mathrm{Be}$. Thus, the sample will return an erroneously old age. Assessing sample purity through mass loss is an inexpensive, easy, and straight forward method to determine sample purity (Brown et al., 1991; Kohl and Nishiizumi, 1992).

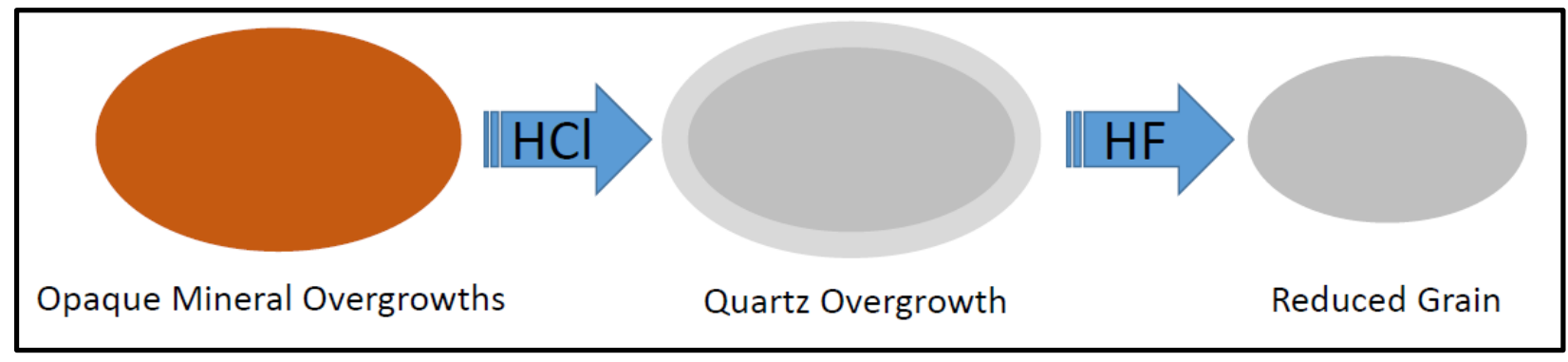

Figure 2.5 Mineral overgrowth removal

The importance of quartz leaching is shown diagrammatically here. Primary grains are coated by minerals produced postor syn-depositionally. These minerals mantle the primary quartz core and contain a host of different elements. If this material is not fully removed before a sample is digested the sample will have a very high overall ion load which may produce issues while the sample progresses through chemistry and/or when it is being measured by and accelerator mass spectrometer.

\section{$\underline{2.2 .2}$ Context for work}

The general sample purification guidelines are described in Kohl and Nishiizumi (1992) and Brown et al. (1991). These two studies form the foundation of chemical sample preparation for the terrestrial cosmogenic nuclide community. While these studies broadly outline the type of acids to use, the duration of sample purification for material, and the overall mass loss to remove meteoric ${ }^{10} \mathrm{Be}$, they do not explicitly address sample grain size below $500 \mu \mathrm{m}$ or track major elements, ${ }^{9} \mathrm{Be}$, or ${ }^{10} \mathrm{~B}$.

Kohl and Nishiizumi (1992) and Brown et al. (1991) focus their studies on grain sizes between $\sim 2 \mathrm{~mm}$ and $500 \mu \mathrm{m}$ with an average size of $\sim 700 \mu \mathrm{m}$. Direct discussion relating to the 
purification of finer material is virtually absent from both studies. Kohl and Nishiizumi report working with grain sizes down to $70 \mu \mathrm{m}$ and simultaneously report they discarded the fine material when cleaning samples. Brown et al. (1991) state care is needed when working with fine material. This thesis is an effort to clarify that statement.

Brown et al. (1991) report that sample mass loss of $\sim 20 \%$ is enough to remove meteoric ${ }^{10} \mathrm{Be}$ from the samples while Kohl and Nishiizumi (1992) report sample purification is confirmed by eye. As-of-yet no clear quantification of major element removal has been done to document the sample purification process for very-fine gained monomineralic quartz samples.

Other chemical treatment methods exist which can conservatively address obtaining and purifying monomineralic quartz (Mifsud et al., 2013). The hot phosphoric acid method is sufficient to remove contaminating silicates (feldspar and biotite) while leaving quartz relatively unaltered. The hot phosphoric acid method is a viable alternative for obtaining clean quartz, however the end product (monomineralic quartz) still needs to be purified. Additionally, while this method is more conservative it does digest a small amount of the quartz as it reacts (Hamada, 1964), and fine-grained material can be difficult to extract from the resulting slurry. Even the small amount of quartz dissolved by this method is problematic for material with fine native grain size. For instance, if hot phosphoric acid is used to digest the interstitial material holding large mineral agglomeration together (as is found in crushed greywacke), it will also digest the fine quartz being liberated. It is, therefore, imperative to restrict the sample to only the native grain size or smaller and the material must be obtained by means other than acid dissolution. 


\subsection{Uses of terrestrial cosmogenic nuclides}

Terrestrial cosmogenic nuclides, such as ${ }^{10} \mathrm{Be}$, are reliable and robust tools to date landforms and calculate bedrock erosion rates (Lal, 1991; Gosse and Phillips, 2001; Balco, 2011). Terrestrial cosmogenic nuclides offer invaluable insight into the long-term development of landscapes. This section explores exposure dating, burial dating, and erosion rate calculation; the three different approaches typically employed to answer landscape evolution and surface process questions.

\subsubsection{Simple and complex exposure systems}

Exposure dating in Antarctica is complicated by several processes that make both sampling and age interpretation challenging (Todd et al, 2010; Balco et al., 2019; Goehring et al., 2019). This section will generally explore the processes which complicate simple exposure histories in Antarctica. A simple exposure history is when a clast undergoes a single cycle of deposition and exposure prior to collection and measurement (Figure 2.6A). Material with a simple exposure history is necessary to constrain the thinning history of outlet glaciers in Antarctica from the Last Glacial Maximum to present. A complex exposure history is when material has two or more exposure events. If the ground surface is covered by any obstruction thick enough to block the cosmic ray flux, it will reduce the corresponding production rate (Dunai, 2010). In Antarctica complex exposure histories are commonly produced by the re-advance and rethickening of glacial ice (Figure 2.6B), as well as intermittent snow cover. As a glacier thins again, and an inherited clast is re-exposed it will once again start accumulating terrestrial cosmogenic nuclides (Figure 2.6C). The different production rates and half-lives of nuclides can provide information on how long material was buried. 

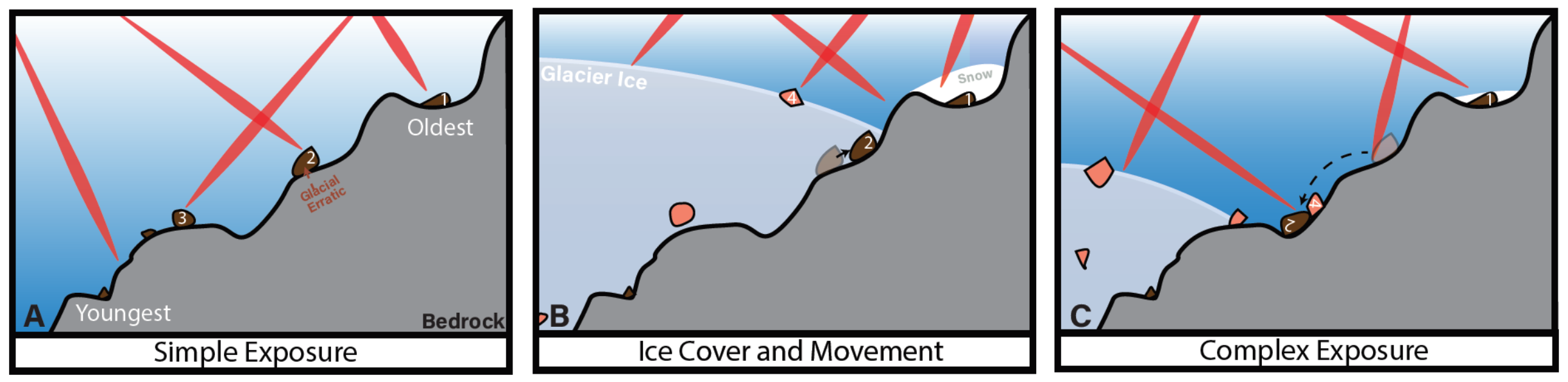

Figure 2.6 Simple versus complex exposure

This figure shows a schematic timeseries of the progression from a simple exposure system to a complex exposure system after one glacial re-advance. A) Erratics 1-3 were deposited in the last glacial thinning event. The clast in the top right (\#1) has the longest exposure history and is the oldest erratic while the clast in the bottom left (\#3) has the shortest exposure history and is the youngest erratic. Each erratic has its own unique simple exposure history in this time step progressing from upper right (oldest) to lower left (youngest). B) The simple exposure erratics are covered as the glacier re-thickens and as snow accumulates in low points (\#1). Under warm-based ice conditions the glacier can re-entrain previously deposited material, notice erratic \#3 is gone. In figure B the re-thickening of the glacier results the re-entrainment of some of the glacial erratics which may be deposited on another nunatak downstream. Under cold-based ice conditions the glacier will not re-entrain previously deposited material but may slightly move the sediment (\#2). In Figure B a glacial erratic has only slightly been moved. The glacial re-advance will also bring new material from up glacier to this location (\#4). C) As the glacier re-thins it deposits new glacial erratics. The newly deposited material is intermingled with previously exposed rocks. Glacial erratics can also roll which may reset the exposure clock for the sample (\#2). 


\section{$\underline{2.3 .2}$ Exposure dating}

Almost any rock surface that is stable and continuously exposed to cosmic ray flux can be dated using any of several radiogenic (e.g. ${ }^{10} \mathrm{Be},{ }^{14} \mathrm{C},{ }^{26} \mathrm{Al},{ }^{36} \mathrm{Cl}$ ) and/or stable (e.g. ${ }^{3} \mathrm{He}$ and ${ }^{21} \mathrm{Ne}$ ) cosmogenic nuclides. The production rates and production pathways are well understood for several nuclides (Gosse and Phillips, 2001). The exposure age of a rock is calculated by the known total nuclide concentration obtained through accelerator mass spectrometry. Stable and radiogenic nuclides with sufficiently long half-lives, such as ${ }^{10} \mathrm{Be}$ and ${ }^{26} \mathrm{Al}$, require a large amount of erosion to reset the surficial signal. In Antarctica this may be accomplished by glacial re-advances eroding several meters of bedrock. If the surface is not totally reset, it will give an apparently old sample age. Other radiogenic nuclides with short half-lives, such as insitu ${ }^{14} \mathrm{C}$, can be reset during sufficiently long glacial re-advances simply by burial of ice leading to total radiogenic decay of all ${ }^{14} \mathrm{C}$ contained in the rock.

\subsubsection{Calculations used to estimate exposure ages}

Exposure ages, burial ages, and erosion rates are all calculated by the derivation of a general formula shown below (equation 2.1). This general equation is the simple case which assumes constant erosion and constant cosmic ray flux.

$$
N(z, t)=N(Z, 0) e^{-\lambda t}+\frac{P(0)}{\lambda+\frac{\rho \varepsilon}{\Lambda}} e^{\frac{-z x \rho}{\Lambda}} *\left(1-e^{-\left(\lambda \frac{\rho \varepsilon}{\Lambda}\right) t}\right)
$$

The variable $N(z, t)$ is the initial sample concentration at $\mathrm{z}$ depth, $N(z, 0)$ is the initial number of atoms, called inheritance, (atoms $\mathrm{g}^{-1}$ ) in the rock surface at depth $\mathrm{z}, \lambda$ is the nuclide specific decay constant, $\rho$ is the density of the rock $\left(\mathrm{g} \mathrm{cm}^{-3}\right), \mathrm{t}$ is time $(\mathrm{yr}), \varepsilon$ is the local erosion rate $\left(\mathrm{m} \mathrm{Myr}{ }^{-1}\right), \Lambda$ is the attenuation length $\left(\mathrm{g} \mathrm{cm}^{-2}\right)$, and $\mathrm{P}(0)$ is the nuclide production rate at the surface (atoms $\mathrm{g}^{-1} \mathrm{yr}^{-1}$ ). 
If the sample does not have a prior exposure history the first term simplifies to the following equation.

$$
N(z, t)=\frac{P(0)}{\lambda+\frac{\rho \varepsilon}{\Lambda}} e^{\frac{-z x \rho}{\Lambda}} *\left(1-e^{-\left(\lambda \frac{\rho \varepsilon}{\Lambda}\right) t}\right)
$$

Exposure ages are calculated by the concentration of a given nuclide that was not present in the mineral prior to exposure to the secondary cascade. Assuming a sample has no erosion $(\varepsilon=0)$, the erosion exponent resolves leaving only the time component in the simplified general formula (eq. 2.2) seen below in (eq. 2.3). Equation three also assumes that the sample was collected at the surface $(\mathrm{z}=0)$ which resolves depth dependency. This equation is used to calculate the exposure age of a radiogenic nuclide (e.g. ${ }^{10} \mathrm{Be},{ }^{14} \mathrm{C}$, and ${ }^{26} \mathrm{Al}$ ) (Brown et al., 1991; and Balco, 2011).

$$
N=\frac{P}{\lambda}\left[1-e^{-\lambda t}\right]
$$

The variable $\mathrm{N}$ is the nuclide concentration (atoms $\mathrm{g}^{-1}$ ), $\mathrm{P}$ is the production rate for the nuclide (atoms $\left.\mathrm{g}^{-1} \mathrm{yr}^{-1}\right)$ in the target mineral, $\lambda$ is the decay constant for the desired nuclide $\left({ }^{10} \mathrm{Be}\right.$ is 4.99 $\times 10^{-7} \mathrm{a}^{-1} \mathrm{yr}^{-1}$ (Chmeleff et al., 2010) and ${ }^{26} \mathrm{Al}$ is $9.63 \times 10^{-7} \mathrm{a}^{-1} \mathrm{yr}^{-1}$ (Goethals et al., 2009)), and $\mathrm{t}$ is the exposure time (yr). This equation can be rearranged to solve for time given a known concentration (Balco, 2011).

A similar equation is used to calculate the exposure age for stable nuclides (e.g. ${ }^{3} \mathrm{He}$ and ${ }^{21} \mathrm{Ne}$ ) (Brown et al., 1991). As stable nuclides do not decay, the decay constant $\lambda$ is removed to produce equation 2.4 . 


$$
N=P t
$$

The variable $\mathrm{N}$ is still the nuclide concentration (atoms $\mathrm{g}^{-1} \mathrm{yr}^{-1}$ ), $\mathrm{P}$ is the production rate for the nuclide (atoms $\mathrm{g}^{-1} \mathrm{yr}^{-1}$ ) in the target mineral, and $\mathrm{t}$ is the exposure time (yr).

\section{$\underline{2.3 .3}$ Burial dating}

Burial dating uses the difference in decay rate between two terrestrial cosmogenic nuclides to determine when a former land surface was buried (Figures 2.7 and 2.8). The burial depth required to fully shield a rock surface is density dependent with a maximum depth of $\sim 30 \mathrm{~m}$. Rapid and total burial below $\sim 30 \mathrm{~m}$ of rock/sediment is necessary to completely inhibit nuclide production from both fast neutrons $\left(\mathrm{n}^{0}\right)$ and deep production by fast muon $\left(\mu^{-} \mathrm{F}\right)$ capture (Dunai, 2010). The most used nuclide combination for this work is ${ }^{10} \mathrm{Be}$ and ${ }^{26} \mathrm{Al}$ because they are both produced in the same mineral, the production ratio for each is not altitude or latitude dependent, the production ratio does not vary greatly with depth from surface, and the halflives are sufficiently different (Dunai, 2010). The ratio between the two nuclides noticeably diverges after $\sim 100,000$ years, with the useful burial dating range from $100 \mathrm{ky}$ to 5 my (Dunai, 2010). If the ground surface has been buried and exposed multiple times then this method can only provide a minimum duration of burial (Dunai, 2010). 


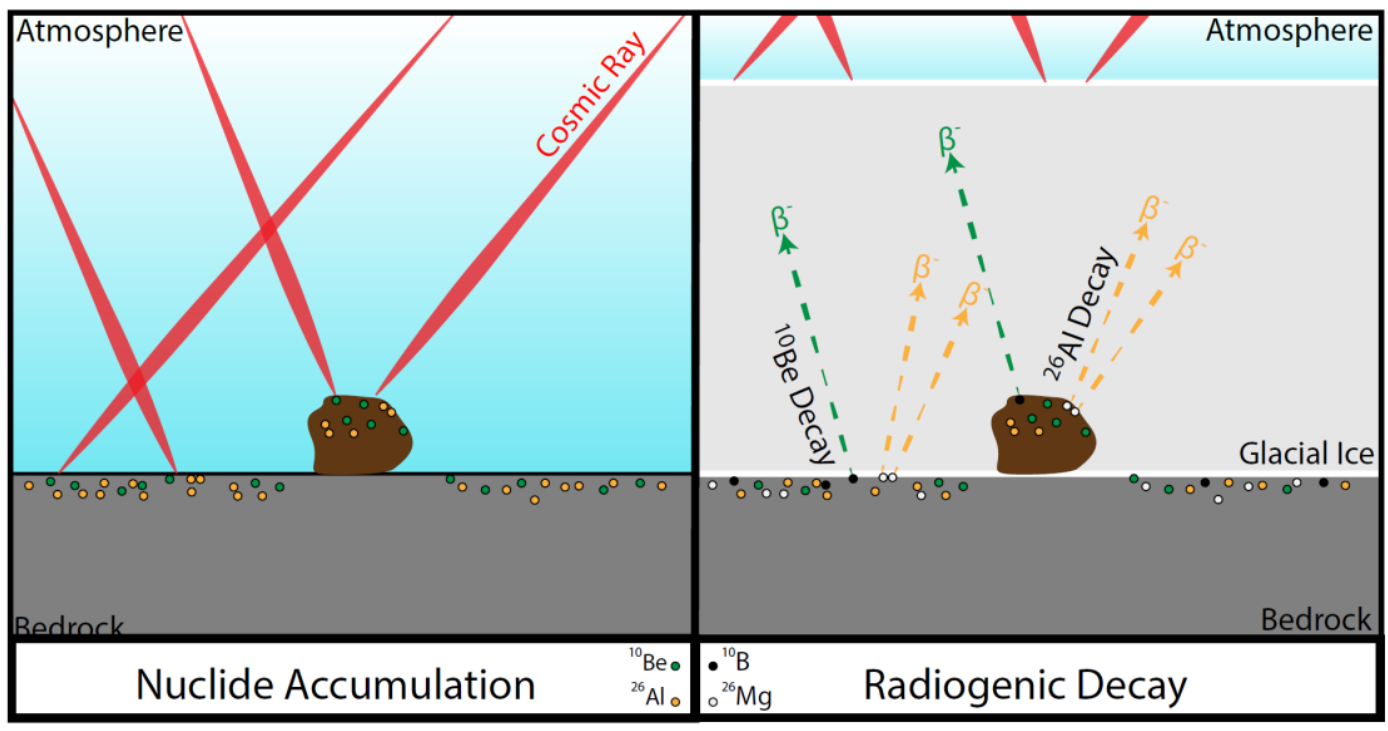

Figure 2.7 Radiogenic decay of nuclides post burial

Left panel: Nuclides accumulating in rock exposed to open sky and bombarded by energetic particles from the secondary cosmic ray cascade (red line). Right panel: If that surface and sediment is rapidly and robustly covered by more than $30 \mathrm{~m}$ of material, total nuclide production will stop. Over time the accumulated nuclides will decay at a predictable rate (green and yellow lines). The differential decay rate can be used to explore the duration the surface and sediment has been buried; ${ }^{26} \mathrm{Al}$ decays $\sim$ twice as fast as ${ }^{10} \mathrm{Be}$. The grey and black points are decayed radiogenic nuclides.

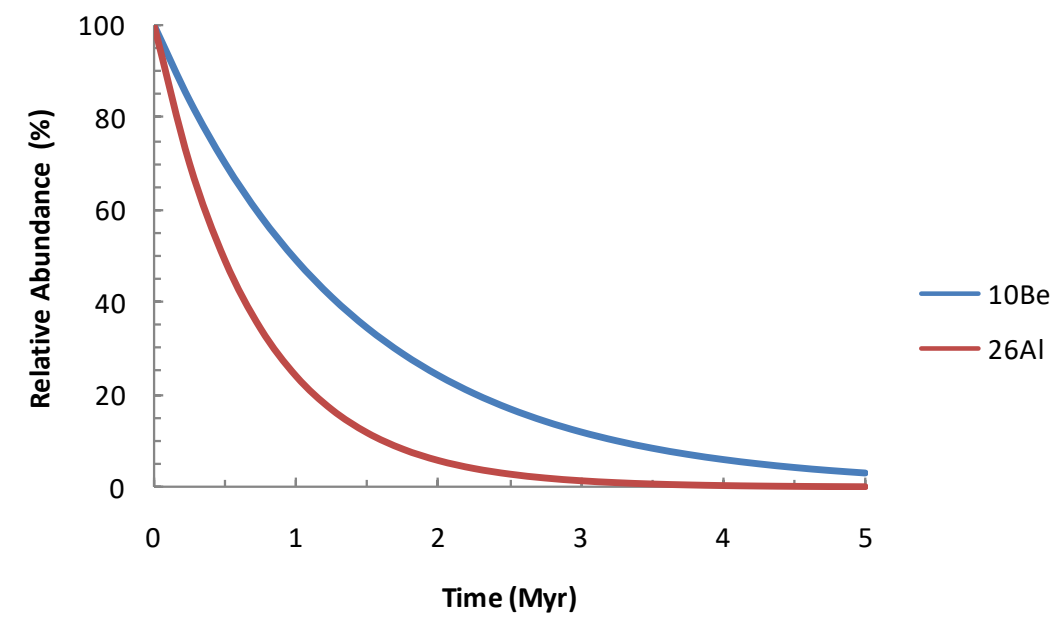

Figure 2.8 Differential nuclide decay

Both ${ }^{10} \mathrm{Be}$ (blue) and ${ }^{26} \mathrm{Al}$ (red) are shown. The half-life of ${ }^{26} \mathrm{Al}(\sim .708 \pm 0.017$ my (Nishiizumi, 2004)) is almost twice as fast as the decay of ${ }^{10} \mathrm{Be}(1.39 \mathrm{my} \pm 0.013 \mathrm{my}$ (Chmeleff et al., 2010; Korshinek et al., 2010)). The differential decay rates are exploited to quantify the burial duration of a surface. This works well in both glaciated (covered by ice) and unglaciated (covered by sediment deposition via many mechanism) situations.

\subsubsection{Calculations used to estimate burial ages}

Burial ages are calculated by exploiting multiple applications of the exposure age equation (eq. 2.3) and the differential decay of radiogenic nuclides. To calculate a burial age two nuclides are required and at least one must be radiogenic. A key component is that the initial 
ratio of the two nuclides must be known. Assuming no post burial nuclide production takes place at depth, equation 2.5 is valid.

$$
t_{b}=\frac{-\ln \left(\frac{R_{A B}\left(t_{b}\right)}{R_{A B}(0)}\right)}{\left(\lambda_{A}-\lambda_{B}\right)}
$$

The variable $t_{b}$ is the burial duration of the sample, $R_{A B}(0)$ is the initial nuclide ratio, $R_{A B}(t b)$ is the measured ratio of the two nuclides, and $\lambda_{A}$ and $\lambda_{B}$ are the decay constants of the two nuclides $\left({ }^{10} \mathrm{Be}\right.$ is $4.99 \times 10^{-7} \mathrm{a}^{-1} \mathrm{yr}^{-1}$ (Chmeleff et al., 2010) and ${ }^{26} \mathrm{Al}$ is $9.63 \times 10^{-7} \mathrm{a}^{-1} \mathrm{yr}^{-1}$ (Goethals et al., 2009).

\subsubsection{Erosion rate}

Calculating an erosion rate using terrestrial cosmogenic nuclides is different than calculating either exposure or burial ages. The surface of the Earth is constantly subject to chemical and physical weathering, which removes material and lowers ground surface relative to the material below. As material is removed from the surface, previously shielded rock enters the nuclide production zone. As that material moves through the nuclide production zone it is constantly irradiated by the cosmic ray flux and accumulates terrestrial cosmogenic nuclides. If that material travels through the production zone quickly it will have a relatively low concentration of terrestrial cosmogenic nuclides, thus a high erosion rate (Figure 2.9). If that material moves through the production zone slowly it will have a high concentration of terrestrial cosmogenic nuclides, thus a low erosion rate (Figure 2.10). The erosion rate is a function of how fast a particle migrates through both the spallogenic and muon $\left(\mu^{-}\right)$capture nuclide production zones (Gosse and Phillips, 2001; Dunai, 2010). Where the erosion rate is less than $10 \mathrm{~m} / \mathrm{My}{ }^{10} \mathrm{Be}$ produced in the muon $\left(\mu^{-}\right)$production zone is minimal at the surface. 
Erosion rates are calculated by assuming a particle at depth passed through both the spallogenic and muon nuclide production zone. As the particle transfers from the shielded zone to the surface it accumulates nuclides. It is worth noting that using glacial erratics to calculate erosion rates violates the assumptions used to evaluate erosion rates via cosmogenic nuclides unless the erratics are more than $2 \mathrm{~m}$ thick.

\section{High Erosion Rate}

Time 1

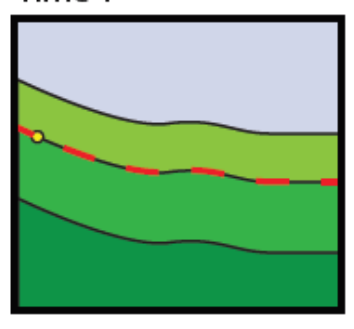

\section{Time 2}

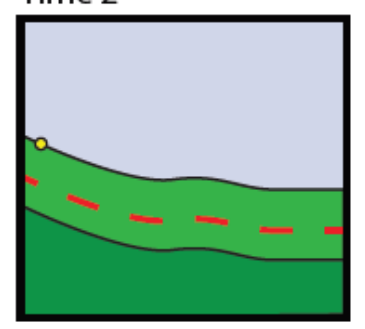

Time 3

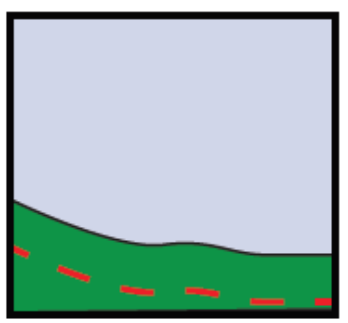

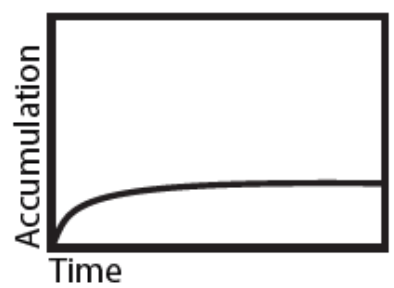

\section{Figure 2.9 High erosion rate}

As the yellow particle at time 1 moves into the bottom of the spallogeninc nuclide production zone (red dashes line) it starts accumulating nuclides. By time 2 the yellow particle has been exposed at the surface having rapidly travelled through the nuclide production zone. By time 3 the particle has itself been eroded away. The graph at the right shows the total nuclide accumulation the yellow particle acquired as it moved from a shielded position through the spallogenic production zone and to the surface. Because of the rapid transit through the production zone it has a low accumulation of measurable nuclides.
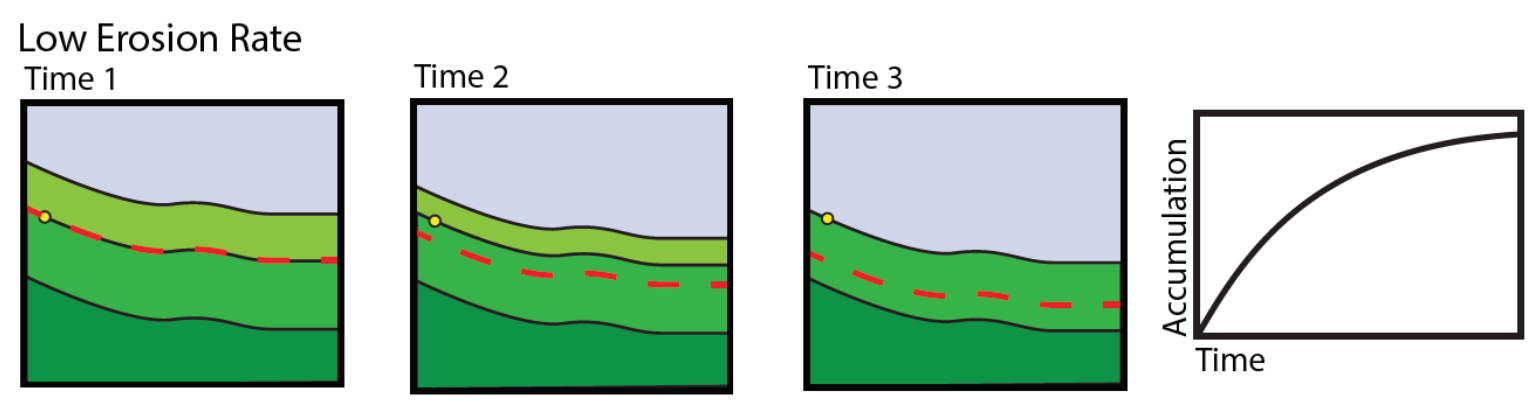

\section{Figure 2.10 Low erosion rates}

As in Figure 2.9 the yellow particle has entered the spallogenic production (red dashes line) zone in time 1. In time 2 the yellow particle is only halfway through the production zone and only at time 3 is the yellow particle exposed at the surface. The graph at the right shows the total nuclide accumulation for the yellow particle acquired as it progressed from a shielded position through the spallogeninc production zone and to the surface. Because of the slow transit through the production zone it has a high accumulation of measurable nuclides.

\subsubsection{Calculations used to estimate erosion rates}

If steady state erosion is $<10 \mathrm{~m} / \mathrm{Myr}$ then the general equation (eq. 2.1) simplifies to one single component and becomes equation 2.6. Erosion rates below $10 \mathrm{~m} \mathrm{Myr}^{-1}$ allow for muons $\left(\mu^{-}\right)$ 
produced below the spallogenic production zone to decay before they reach the surface (Dunai, 2010).

$$
N=\frac{P(0)}{\lambda+\frac{\rho \varepsilon}{\Lambda}}
$$

The variable $\mathrm{N}$ is the total concentration, $\mathrm{P}(0)$ is the surface production rate, $\lambda$ is the decay constant, $\rho$ is the density of the overburden, $\varepsilon$ is the erosion rate, and $\Lambda$ is the attenuation length.

\subsubsection{Differential erosion}

Differential erosion is commonly discussed on two different scales. It is invoked to discuss both major regional differences in landforms, as well as the outcrop scale details of surface form (Waters, 1957). Erosion is governed by several geological and climatic controls (e.g. joint spacing, rock type, freeze thaw cycles, etc.) specific to individual rock outcroppings and locations. At the outcrop scale, two different lithologies can be exposed simultaneously and over time diverge with respect to the top surface position (Figure 2.11). By dating the resistant rock and knowing the change in the top surface, the erosion rate for a non-resistant rock can be calculated.

\section{Differentail Erosion Rate}
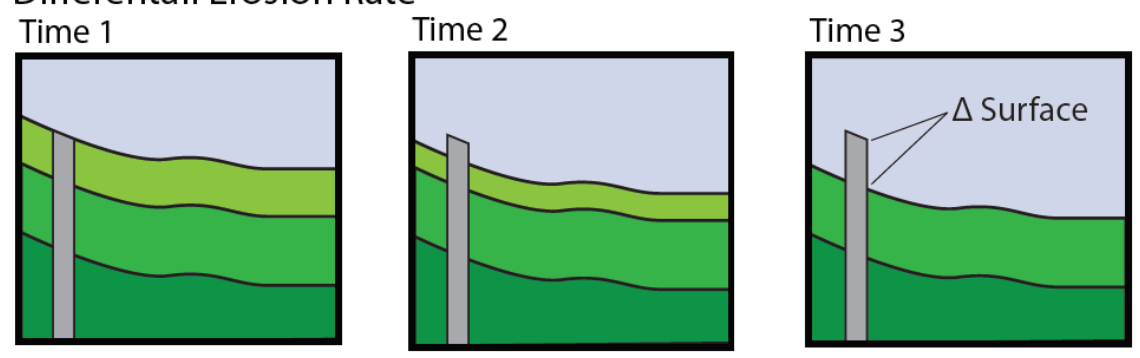

Figure 2.11 Differential erosion

At time 1 the land surface is uniform, neither rock type stands in relief over the other. As time progresses the resistant rock is progressively exhumed as the non-resistant rock erodes faster (time 2 and 3). By dating the resistant rock and knowing the total amount of differential exposure between the two rock types ( $\Delta$ Surface in time 3 ), the erosion rate for the non-resistant rock can be calculated. The change in surface height can be from centimetres to kilometres in height. 
Equation 2.7 is valid if the two rock surfaces were coincident when the surface was first exposed, and that the erosion rate of the resistant rock is comparatively zero.

$$
D_{e}=\frac{\Delta S}{t}
$$

The variable $D_{\mathrm{e}}$ is the differential erosion rate $\left(\mathrm{cm} \mathrm{yr}^{-1}\right), \Delta S$ is the difference between the two top surfaces $(\mathrm{cm})$, and $\mathrm{t}$ is the exposure age $(\mathrm{yr})$.

\subsubsection{Cosmogenic nuclide limitations}

While cosmogenic nuclide dating is a robust tool to evaluate the age of both landforms and sediment deposited at the surface of the Earth, there are several assumptions that are made which need to be addressed: 1) we know the production rate of ${ }^{10} \mathrm{Be}$ and other nuclides, 2) we know the long-term cosmic ray flux (Korte et al., 2011; Lifton et al., 2014), and 3) we assume that secondary radiation is isotropic. The implications are then that cosmic ray flux and secondary radiation is known and understood both spatially and temporally. These assumptions underpin how exposure ages, burial ages, and erosion rates are calculated (Gosse and Phillips, 2001).

Errors associated with sample attributes such as sample thickness, topographic shielding, atmospheric shielding, and sample orientation, are important, but typically account for no more than 2\% uncertainty (Gosse and Phillips, 2001). Measurements taken in the field and in the lab such as the density of the sample, azimuth and inclination of surrounding topography, and strike and dip of inclined surfaces are used to constrain and quantify these limitations (Dunne et al., 1999; Schildgen et al., 2005). The accumulation of meteoric cosmogenic nuclides on 
mineral surfaces is assumed to be totally removed by acid etching and quartz purification (Kohl and Nishiizumi, 1992; Brown et al., 1991; Gosse and Phillips, 2001).

Challenges specific to cosmogenic dating in polar/cold climate and alpine landscapes are the presence of cold-based ice, thick multiyear snow patches, cryoturbation, colluvial processes, and clast recycling. Cold-based ice in Antarctica has the potential to shield and protect previously exposed old erratics without significantly moving them. These samples would then yield an older age than the last time a glacier was present. Large amounts of snow in multiyear snow patches can shield erratics from accumulating nuclides giving them an artificially young age and may produce an error of $10 \%$ or more depending on snow cover persistence (Schildgen et al., 2005) (Figures 2.6 and 2.7). Cryoturbation and colluvial processes can reorient sedimentary deposits and artificially reset the cosmogenic clock by rotating clasts (Figure 2.6C). Clast rotation is a significant issue for cobbles larger than $10 \mathrm{~cm}$ in diameter, because the whole sample will not be processed to extract ${ }^{10} \mathrm{Be}$. Where warm based ice is present clast recycling can be problematic because material can be remobilized. These types of recycled clasts are identical to our target clast and when collected yield an older age than that of the most recent depositional event. There is no way to filter recycled clasts out while sampling. (Figure 2.12). 


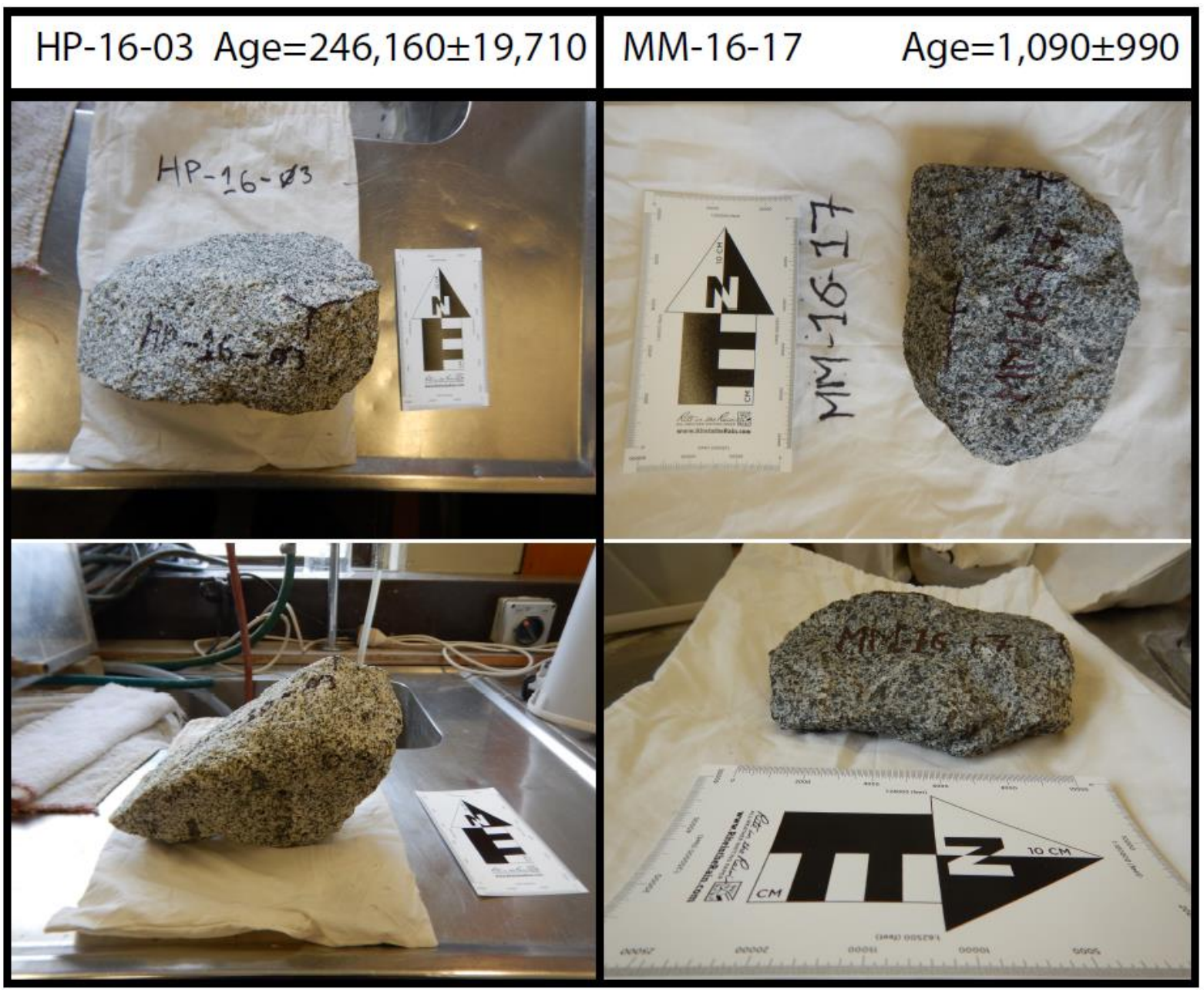

Figure 2.12 Old and young sample comparison

Both samples (left HP-16-03 and right MM-16-17) were collected on unfractured bedrock and displayed the prime characteristics of a shaped erratic. Neither sample show a strong weathering rind, strong granular spallation, or any other indicators of extreme age. But sample HP-16-03 (left set of photos) is 246,160 $\pm 19,710$ years old and sample MM-1617 (right set of photos) is 1,090 \pm 990 years old. As explained in the text, clast morphology is not a reliable indication of sample age. Therefore, statistically significant numbers of samples need to be processed in order to determine the rate, magnitude, duration and timing of ice-surface-elevation changes for outlet glaciers in Antarctica.

To overcome these challenges, strategic sampling procedures are employed to reduce the chance of selecting recycled and reoriented sediment. Additionally, many samples are collected. By focussing collection efforts on glacially shaped erratics prominently perched on bedrock sitting above the local topography we reduce the chance of collecting samples with an anomalously young or old age. By sampling glacially shaped clasts we ensure material has been through the zone of basal traction and is likely derived from a subglacial source. Collecting precariously positioned metastable samples perched on bedrock ensures that the 
clast has not been post-depositionally reoriented or moved significantly. Collecting samples from local high points provides confidence that a clast has not been covered by significant multi-season snowpack. Given this criterion, if many samples are collected then anomalously young and old samples will stand out. Additionally, by producing a large data set a robust statistical analysis can be conducted.

There are three distinct issues prevalent in areas outside of Antarctica which need to be addressed separately because they are less problematic in Antarctica; 1) rock erosion rate, 2) atmospheric thickness, and 3) geomagnetic field strength. Firstly, previously measured erosion rates in Victoria Land are very low, between 5-10 cm/Ma (Nishiizumi et al., 1991; Brook et al. 1995; Ivy-Ochs et al. 1995) and 1-4 m/Ma (Nishiizumi et al., 1991; Van der Wateren et al., 1999). This means material deposited in the last several thousand years is unlikely to be significantly affected by erosive processes but are still critical for calculating exposure ages. Secondly, the mean atmospheric pressure over Antarctica is $20-40 \mathrm{hPa}$ lower than the standard pressure-altitude curve (Radock et al., 1996; Stone, 2000). Which can account for the higher cosmic ray flux in Antarctica. Thirdly, the Earth's magnetic field strength is dramatically reduced a high latitude (Gosse and Phillips, 2001; Smart and Shea, 2009). The reduced magnetic field strength at high latitudes again increases the total cosmic ray flux at the ground surface in Antarctica. Ultimately, high flux rates coupled with low erosion rates means that Antarctica is ideal for cosmogenic nuclide sampling.

\subsection{Ice sheet drainage pathways}

It is necessary to understand outlet glacier and ice stream behaviour in order to interpret the large-scale response of ice sheets to climate forcing. Outlet glaciers and ice streams are critical drainage pathways for large ice sheets, draining the majority of ice from the ice sheet interior 
to the coast (Figure 2.13). An outlet glacier is a glacier which drains an ice sheet through confined topography either a mountain valley or fjord, whereas, an ice stream is a fast-flowing ribbon of ice moving adjacent to significantly slower ice. Any change to these drainage systems directly affects the mass balance of large ice sheets (Bamber et al. 2000; Barry and Yew Gan, 2011).

Several factors affect the stability of outlet glaciers and ice streams including surface and basal mass balance, ice flow dynamics particularly its state of stress with respect to buttressing ice, and bedrock. A number of factors can enhance or inhibit the discharge of ice from a large ice sheet through outlet glaciers and ice streams. These systems drain significant portions of both the East and West Antarctic Ice Sheets. A large outlet glacier or ice stream can drain ice thousands of kilometres inland from its terminus (Weertman, 1974; Bamber et al., 2000; Bamber et al., 2001; Dupont and Alley, 2005; Dupont and Alley, 2006). 


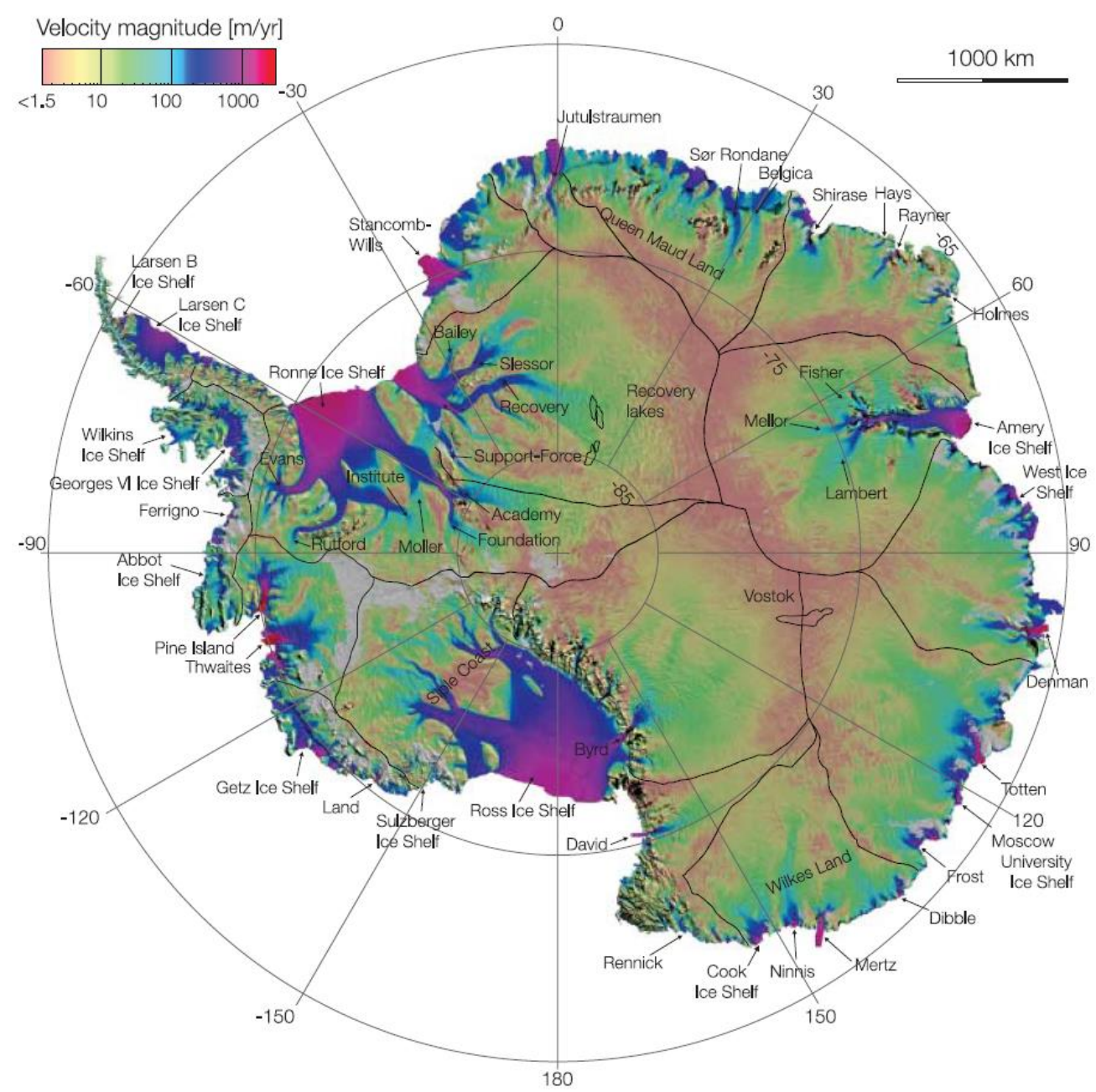

Figure 2.13 Outlet glaciers

The map shows ice velocity for the Antarctic Ice Sheet. The colour red shows ice velocity less than $1.5 \mathrm{~m} \mathrm{yr}^{-1}$ and purple shows ice velocity greater than $1000 \mathrm{~m} \mathrm{yr}^{-1}$. Ice sheet velocity increases at specific locations along the coast due to the presence of an outlet glaciers or ice stream. An outlet glacier is a narrow and fast flowing glacier which drains the ice sheet. Many are marine terminating and are fed by ice from hundreds to thousands of kilometres inland from the mouth of the glacier. This figure is from Rignot et al., 2011.

Ice discharge is stabilised by the presence of an ice shelf. This feature inhibits free and unrestricted ice discharge (Dupont and Alley, 2005; Dupont and Alley, 2006; Fürst et al., 2016;

Reese et al., 2018) thus stabilizing the outlet glacier (Reese et al., 2018). The removal of this resistive force, when an ice shelf collapses, can lead to catastrophic increases in ice discharge, such as were observed in 2002, when the Larson B ice shelf collapsed (Scambos et al., 2004). The loss of buttressing ice can then lead to rapid and catastrophic down wasting of glaciers. 
The interaction of local topography and relatively warm ocean water at the grounding line also plays a critical role in controlling the rate of retreat for individual glaciers. This interaction can produce an intense and potentially irreversible positive feedback. This process is called Marine Ice Sheet Instability (Weertman, 1974: Schoof, 2007a,b) and may be under way in the Amundsen Sea Sector of Antarctica at Pine Island, Thwaites, and Smith glaciers (Joughin et al., 2014; Favier et al., 2014; Ritz et al., 2015; Feldmann and Levermann, 2015; Oppenheimer et al. in IPCC SROCC, 2019). The loss of back stress produces a velocity perturbation that migrates up stream. Dramatic rates of ice surface lowering and significant ice loss occur before the grounding line stabilizes. It is important to note that rapid mass loss can be episodic, and the rate of ice surface elevation change is preserved in sediment it deposits when it is thinning.

The magnitude and rate of thinning can be measured using terrestrial cosmogenic nuclides because as the glacier is discharging ice it is also depositing glacial erratics on newly exposed bedrock adjacent to the thinning glacier. Some of this freshly deposited sediment will not have been exposed at the surface prior to this event, and once deposited will start accumulating terrestrial cosmogenic nuclides. Careful sample selection can reduce but not eliminate material with an inherited signal from prior exposure. A thoughtfully sampled elevation transect perpendicular to a glacier can provide insight into ice elevation changes over the last several thousand years. Chronologies which record large scale ice surface elevation changes are critical to refine the current understanding of outlet glacier response to climate forcing and internal feedbacks. They provide information on the rate and style of retreat and can be used to evaluate ice sheet models which simulate ice sheet change through time at a regional or continental scale. 


\subsubsection{Steady state ice flow}

This section is not intended as a thorough review of all driving and resisting stresses in a glacier but will consider normal and shear stresses along with Glen's flow law and the role of basal meltwater. This background is required to help interpret changes in outlet glaciers through time which I reconstruct in (Chapters, 5 and 6). For a comprehensive review of ice sheet model equations please see Pattyn (2003).

Several types of glaciers exist the largest of which are ice sheets. The two ice sheets on Earth are in Greenland and Antarctica, both of these glaciers drain ice from interior regions to the ocean. Antarctica is a complex ice sheet with two distinct regions separated by the Transantarctic Mountains. The East Antarctic Ice Sheet is grounded above sea level with the exception of the Wilkes Subglacial Basin and the Recovery Basin and the West Antarctic Ice Sheet is largely grounded below sea level. Both marine based and continental based ice sheets discharge ice from the ice sheet interior to the ocean through ice streams, outlet glacier, and ice shelves. Marine based ice sheets, outlet glaciers, and ice streams may be inherently unstable.

The mass balance of a glacier is largely dependent on the climatic conditions present and the amount of snow accumulation high on a glacier and areas of ice loss low on a glacier (Sugdon and John, 1976; Bennett and Glasser, 2009). For the large Antarctica ice sheet, the highest accumulation is located on the Antarctic Peninsula followed by the coastal regions of West Antarctica and a significant drop in accumulation along the coast of East Antarctica while snow accumulation in the ice sheet interior is extremely low (Van den Broeke et al., 2011) (Figure 2.14). The mass balance of a glacier is important because it is a major factor that determines if a glacier will grow, shrink, or remain at a steady state 


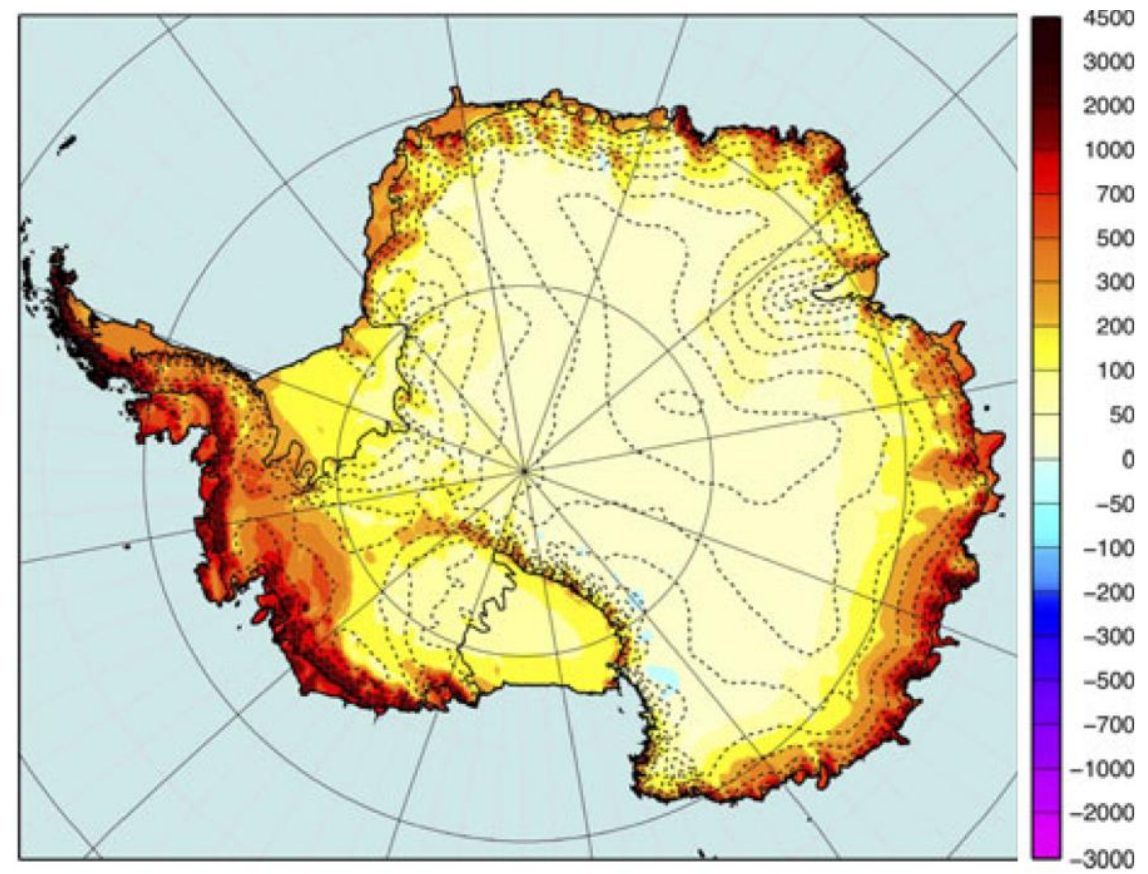

Figure 2.14 A schematic diagram of an ice sheet

This figure shows surface mass balance from 1989-2009 in $\mathrm{kg} \mathrm{m}^{-2} \mathrm{yr}^{-1}$. The data are from RACMO2. Figure was modified from Van den Broeke et al., 2011.

A glacier flows due to the deformation of ice under the direct effect of gravity and the glaciers own surface gradient which are explored below (Figure 2.14). Glacial ice flows as a material (strain) response to the applied stresses. The ductile strain response of ice also known as creep is reasonably approximated by Glen's flow law (Glen, 1952) seen in equation 2.8.

$$
\varepsilon=A \tau^{n}
$$

Where $\varepsilon$ is the compressive strain rate, $\mathrm{A}$ is a constant which is dependent on crystal orientation, ice temperature, and sediment content among other factors, $\tau$ is the basal shear stress, and $\mathrm{n}$ is a constant (Glen, 1952). The strain rate controls the type of deformation, either brittle or ductile, present at specific points on and in the glacier. Locally the strain rate can be high enough that creep is no longer the main deformation mechanism and the ice will fold or fault (Bennet and Glasser, 2009). 
Brittle and ductile deformation allows ice to move from the zone of accumulation to the zone of ablation/ice loss, with other key processes enhancing or inhibiting the flow of ice. The stresses which govern glacial flow are represented in several equations. In the simple case of a glacier resting on relatively flat bedrock, the basal normal stress $(\sigma)$ is given by equation 2.9.

$$
\sigma=\rho_{i} g h
$$

Where $\rho_{i}$ is the density of ice (glacial ice $\sim 910 \mathrm{~kg} \mathrm{~m}^{-3}$ ), $\mathrm{g}$ is the acceleration due to gravity on Earth $\left(\sim 9.8 \mathrm{~m} \mathrm{~s}^{-2}\right)$, and $\mathrm{h}$ is the thickness of the glacier (m). For a glacier on sloping topography equation 2.9 is modified to determine the basal shear stress $\left(\tau_{b}\right)$ and is shown in equation 2.10 (Nye, 1952).

$$
\tau_{b}=\rho_{i} g h * \sin (\alpha)
$$

Where $\alpha$ is the surface slope, and the other variables from equation 2.9 stay the same. The shear stress can also be solved for internal shear stress, as is shown in equation 2.11 (Nye, 1952).

$$
\tau_{b}=\rho_{i} g(s-z) * \sin (\alpha)
$$

Where the $\mathrm{s}$ is the surface elevation, and $\mathrm{z}$ is the elevation inside the glacier. The state of stress changes from the top to the bottom of the glacier. The basal shear stress is also influenced by the presence or absence of water. Liquid water is a major control on ice-flow dynamics, the frictional interaction between the glacier and its bed, the strength of subglacial sediment, and 
it also reduces the effective normal stress $(\sigma)$. Equation 2.12 is used to evaluate the magnitude of support from liquid water at the base of a glacier (Clarke, 2005).

$$
p_{e}=\left(\rho_{i} g h\right)-p_{w}
$$

Where $p_{e}$ is the effective pressure, and $p_{w}$ is the water pressure present at the base of the glacier. The amount of sub-glacial water is regulated by a combination of processes within the ice-bed interface and the porosity and permeability of the substrate underlying the glacier. The flow and distribution of sub-glacial water is governed by pressure gradients as shown in equation 2.13 (Clarke, 2005).

$$
\Phi_{w}=p_{w}+\rho_{w} g z
$$

Where $\Phi_{w}$ is the fluid potential, $p_{w}$ is the water pressure present, $\rho_{\mathrm{w}}$ is the density of water, $\mathrm{g}$ is the acceleration due to gravity, and $\mathrm{z}$ is the bed surface elevation. When $\Phi_{w}$ is zero water can pond under a glacier. The sub-glacial water pressure gradients are largely controlled by the surface topography (Clarke, 2005). The amount of sub-glacial water varies on diurnal to annual time scales and the surface mass balance (Bindschadler, 1983; Iverson, 1995).

The ice sheet-ice/shelf transition is a complex interaction between the vertical stresses of a grounded ice sheet and the longitudinal and lateral stresses of the floating ice shelf (Schoof, 2007a,b; Gagliardini et al., 2010) (Figure 2.15). The lateral resistance of an ice shelf can be described by the body force equation shown in equation 2.14 .

$$
f=-K|u|^{m_{l r}-1} u
$$


Where $\mathrm{f}$ is the body force, $\mathrm{K}$ is the lateral resistance coefficient, $\mathrm{m}_{\mathrm{lr}}$ is the lateral resistance exponent, and $\mathrm{u}$ is the velocity vector. In full stokes modelling experiments show that in a complex way ice shelves buttress ice sheets (Gagliardini et al., 2010). The complex relationship between ice shelves and ice sheets also affects how the grounding line responds to changes in heat flux. Gagliardini and others (2010) suggest that as a consequence of melting the sea may encroach on the grounding line is overly simplistic an in some cases wrong. The distribution of melting is a key factor in controlling grounding line behaviour (Gagliardini et al., 2010).

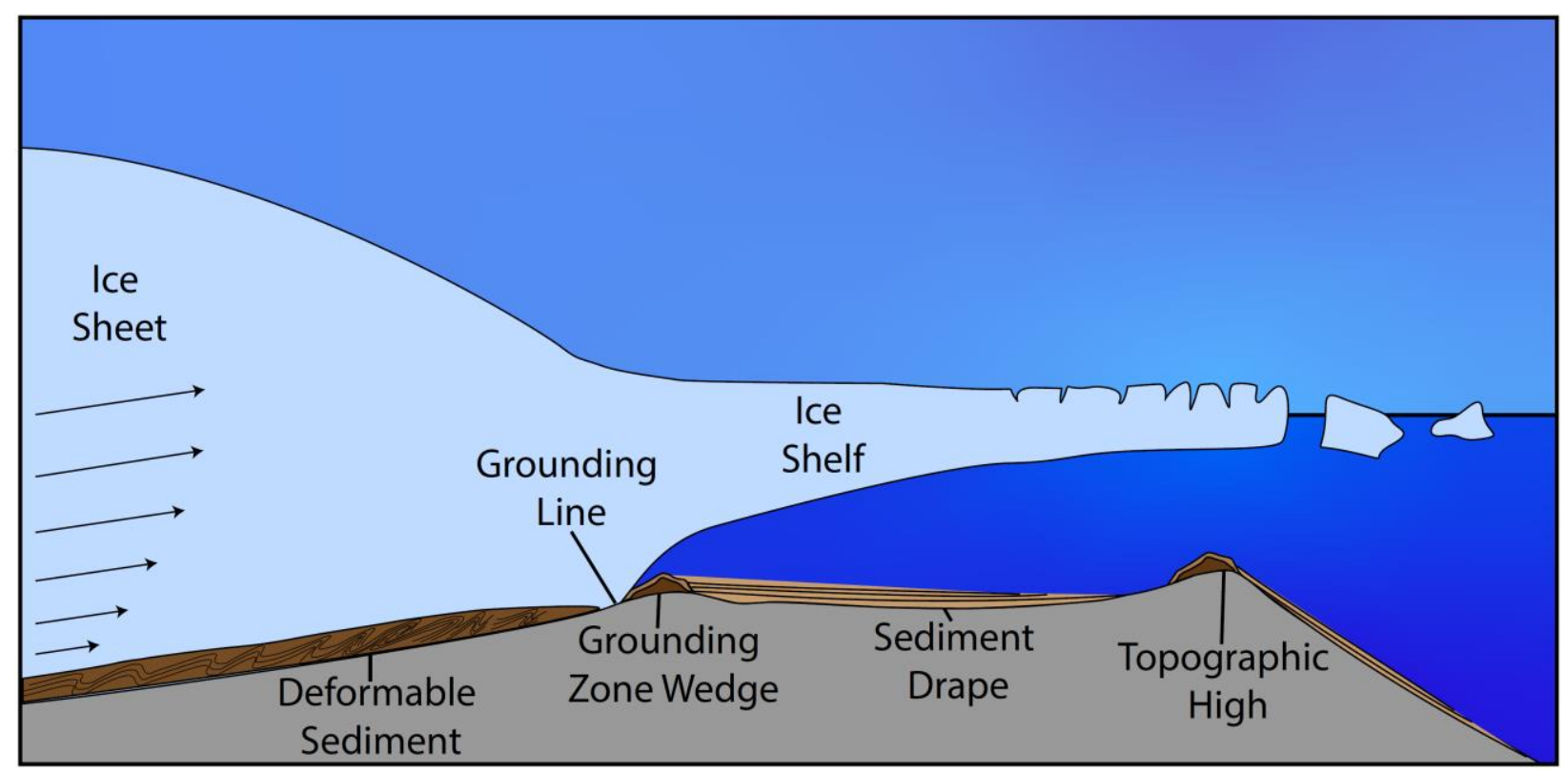

Figure 2.15 Cross section of a marine terminating glacier

This figure shows the configuration of an ice sheet with an ice shelf attached. The vertical stresses of the ice sheet reduce to zero when the ice starts to float in the ice shelf.

\subsubsection{The marine ice sheet instability mechanism}

The Antarctic and Greenland ice sheets store most of the land-based frozen fresh water on Earth. Melt from these large ice sheets will contribute to sea-level rise through the $21^{\text {st }}$ century and beyond (Golledge et al., 2015; Oppenheimer et al. in IPCC SROCC, 2019). Approximately one third of the Antarctic Ice Sheet is grounded below sea level and most of the ice-sheet margin terminates into the ocean. The marine terminating portions of ice sheets 
may be susceptible to dynamic instability which may lead to rapid ice loss and add to sea-level rise (Oppenheimer et al. in IPCC SROCC, 2019). The marine ice sheet instability (Figure 2.16) mechanism is discussed below.

For marine terminating glaciers, grounded ice stability is a balance between geographic features, ice shelves/ice tongues, and climate forcings (Figure 2.15), some of which promote stability (e.g. ice shelves, high-points on the sea floor, etc.), and some of which promote instability (e.g. an influx of warm ocean water at the grounding line, topographic slopes which dip towards the ice sheet (retrograde slopes), etc.). Many of the large outlet glaciers and ice streams flow into ice shelves, pinned on bathymetric highs, or are floating ice tongues that are confined in fjords. These features restrain the outflow of upstream grounded ice stabilizing outlet glaciers, ice streams, and ultimately ice sheets.

Ice shelves are the key factor controlling Antarctic Ice Sheet dynamics (Furst et al., 2016; Reese et al., 2018). Ice shelf stability is reduced by relatively warm ocean water, surface melt, rainwater, and flexural stresses enhancing hydrofracturing (Macayeal and Sergienko, 2013; Banwell et al., 2013). The dynamic response of the Antarctic Ice Sheet over the next few centuries to millennia will be determined in part by changes in ice shelf geometry and in turn, grounding line stability. If the grounding line retreats down a bedrock slope dipping towards the ice sheet interior (retrograde slope), it may experience a positive feedback which enhance thinning and retreat and inhibits stabilization (Figure 2.16) resulting in progressively more ice discharge into the ocean. This positive feedback is known as marine ice sheet instability (Weertman, 1974: Schoof, 2007a, b). This self-sustaining positive feedback is not unstoppable or inevitable for all marine based glaciers (Gomez et al., 2015). Local conditions such as the isostatic adjustment, bed shape, bed geometry, lateral shear stress from the fjord wall, 
gravitational effects on local sea level, and ice shelf pinning points govern the retreat and thinning behaviour of marine-based glaciers (Gomez et al., 2015) and together one or more of these effects may result in stabilization or enhancement of a retreating grounding line.
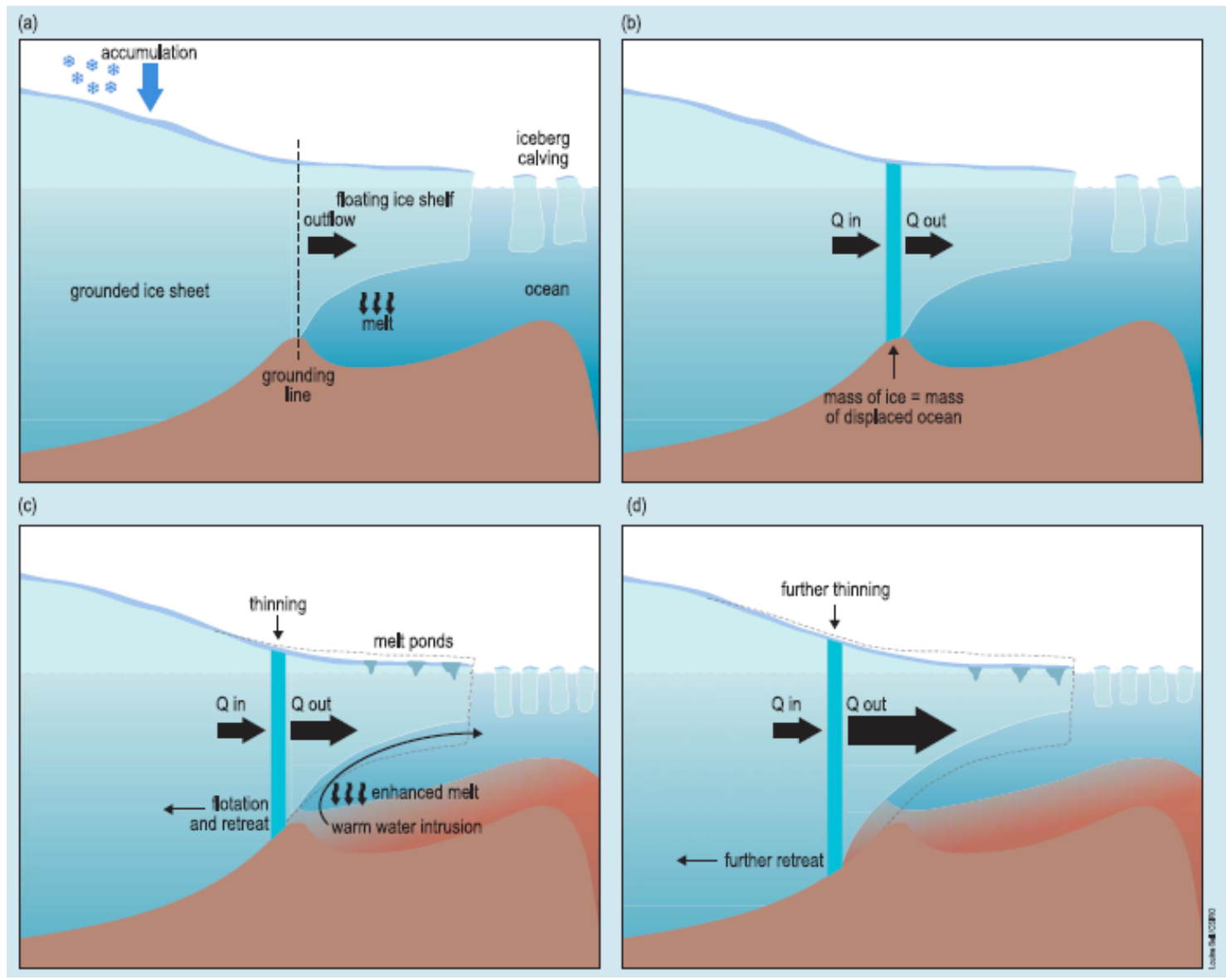

\section{Figure 2.16 Marine ice sheet instability}

A) Shows a marine terminating glacier in a stable configuration with its grounding line perched on a topographic high. B) Shows that in a stable configuration steady-state ice flux is equal in front of and behind the grounding line. C) Warm water incursion increase thermal erosion of the basal ice around the grounding line and on the bottom of the ice shelf. As this process starts to initiate the grounding line moves off the topographic high point and starts to retreat down a retrograde slope. As grounding line retreat occurs, the driving stresses also increase due to a steeper surface slope. D) More warm water thermally erodes ice at the grounding line, which makes it migrate farther down the retrograde slope, thus expanding the cross-sectional area an increasing the ice flux. The positive feedback will continue until the glacier finds a new stable configuration. This figure was taken from Church et al., 2013.

Marine ice sheet instability may be under way in the Amundsen Sea Embayment of West

Antarctica at Pine Island, Thwaites, and Smith glaciers (Rignot et al., 2014; Joughin et al.,

2014; Favier et al., 2014; Ritz et al., 2015; Feldmann and Levermann, 2015; IPCC SROCC,

2019) with thinning rates of between 3 and $5 \mathrm{~m} \mathrm{yr}^{-1}$ for Pine Island and Smith glaciers and 
associated grounding line retreat with between 30 and $35 \mathrm{~km}$ in $~ 20$ years (Rignot et al., 2014). The thinning rate of Thwaites Glacier is lower and more variable because it is not confined by a deep trough but expands over a $\sim 100 \mathrm{~km}$ area with complex bed topography (Rignot et al., 2014). The thinning and retreat of these glaciers is likely to be irreversible (Favier et al., 2014; Feldmann and Levermann, 2015; Arneteh et al., 2017; Oppenheimer et al. in IPCC SROCC, 2019) and, in time, may affect the whole of the West Antarctic Ice Sheet (Feldmann and Levermann, 2015). Grounding line retreat in the Amundsen Sea embayment was likely instigated and sustained by an increase in thermal erosion at the grounding line via relatively warm water interactions with the base of each glacier (Jacobs et al., 2011; Arneth et al., 2017; Assmann et al., 2019).

Rapid thinning and retreat of outlet glaciers connected to large ice sheets has the potential to dramatically raise sea level. These events can be abrupt and increase sea level by tens of metres in a short time (Fairbanks, 1989; Bard et al., 1996; Hanebruth et al., 2000; and Webster et al., 2004). Understanding instability in marine terminating glaciers is critical for future sea-level rise projections.

\subsubsection{Meltwater pulse-1a}

Data collected from drowned coral reefs shows that rapid discharges of fresh water derived from ice-sheets have dramatically increased paleo-sea level (Fairbanks, 1989; Bard et al., 1996; Hanebruth et al., 2000; and Webster et al., 2004). These fresh-water discharge events, called melt-water-pulses, may not be solely attributable to Northern Hemisphere deglaciation over the last $\sim 19,000$ years despite the total collapse of several Northern Hemisphere ice sheets. The largest of these discharge events, Meltwater pulse-1a (MWP-1a), occurred between $\sim 14,650$ and $\sim 14,310$ years ago. The magnitude of sea-level rise from this event is 
between 8.6 and $14.6 \mathrm{~m}$ of change with a maximum duration of $\sim 350$ years (Deschamps et al., 2012; Liu et al., 2016). It is still uncertain whether Antarctica was a significant source of water for Meltwater pulse-1a (Clark et al., 2002; Liu et al, 2016). A spatially integrated data set of iceberg rafted debris from the Scotia Sea provides some evidence that Antarctica may have undergone eight different calving events. The most intense event, AID6, peaks from $14.8 \mathrm{ka}$ 14.4 ka, and is coincident with Meltwater pulse-1a (Weber et al., 2014). However, the relationship between Meltwater pulse-1a and rapid thinning or grounding line retreat of outlet glaciers in Antarctica is not resolved. Ice sheet modelling suggests that the Ross Sea Sector of Antarctica could have been a source of water related to Meltwater pulse-1a (Golledge et al, 2014). However, geologic evidence from the McMurdo Sound area indicates that the Ross Ice Shelf was in a stable configuration until $12.8 \mathrm{ka}$ (Hall et al. 2015). Critically, neither the total extent of grounded ice (Denton and Hughes, 2000; Shipp et al., 1999), nor the pattern of grounding line retreat in the Ross Embayment is not well constrained (Conway et al., 1999; Ackert et al., 2008; Golledge et al., 2014; Halberstadt 2016). Terrestrial geologic evidence does not support a Ross Sea contribution to Meltwater pulse-1a (Spector et al., 2017). However, undated grounding zone wedges in the Wales Deep and Glomar-Challenger Basin (Halberstadt et al., 2017) coupled with ice-sheet modelling scenarios (Ackert et al., 2008, Golledge et al., 2016) indicate that grounding line retreat in the Central Ross Embayment may have initiated $~ 14$ kya. Ice from the East Antarctic Ice Sheet supplied more than half of the ice which filled the Ross Embayment during the Last Glacial Maximum (Anderson et al., 2014). Further clarification of the relationship between rapid sea-level rise and Antarctic ice sheet response is needed. 


\subsubsection{Ross Sea deglaciation history}

Three hypotheses colloquially known as the "Swinging Gate" (Conway et al., 1999), "Saloon Doors" (Ackert et al., 2008; Golledge et al., 2014), and the "Marine Based" (Halberstadt 2016) aim to resolve the pattern and timing of deglaciation in the Ross Sea since the Last Glacial Maximum. These scenarios have varying levels of complexity and are described in more detail below.

The "Swinging Gate" scenario presents the idea that grounded ice in the Ross Sea is pinned at Roosevelt Island in the eastern Ross Sea and rapidly retreated south along the Victoria Land coast in the western Ross Sea (Figure 2.17A). The main implication is that over time an ice shelf and eventually open water would have moved from north to south following the grounding line (Conway et al., 1999).

The "Saloon Doors" hypothesis is more complex and starts with grounding line retreat in the central Ross Sea (Figure 2.17B). After the initial phases of retreat the grounding line progresses radially to the east, south, and west (Ackert et al., 2008). A more complex version of the "Saloon Doors" hypothesis, called the "Marine Based" hyopothesis (Figure 2.17C), more directly involves the role of sea-floor topography as a key control on the rate and style of grounded ice retreat in the Western Ross Sea (Golledge, 2014; McKay et al., 2015; Halberstadt et al, 2016). This idea builds on the "Saloon Doors" concept and recognizes submarine topography as a significant control on the style of retreat. The main conclusion to draw from this scenario is that grounded ice retreated from the central Ross Sea, in a landward direction. This scenario allows for small ice shelves to be preserved at submerged high topography and at some locations along the coast. The high topography allows for a piecemeal retreat of buttressed ice along the coast. The implications are that individual outlet glaciers 
along the Victoria Land coast would record asynchronous thinning and retreat due to specific and local topographic influences.

The greater implication of these scenarios is that outlet glaciers along the Victoria Land coast where grounded ice was present may have been significantly impacted by the removal of back stress. If so as ice flux through outlet glaciers along the Victoria Land would increase as grounded ice in the Ross Sea retreated south. Depending on the total extent of either grounded or floating ice in the Ross Sea and the rate of retreat, a significant amount of freshwater could have been discharged into the ocean, affecting both climate and sea level. Additionally, the full extent of grounded ice in the Ross Sea is not well constrained with an $\sim 150 \mathrm{~km}$ discrepancy in the maximum grounding line position during the last glacial maximum (Shipp et al., 1999; Denton and Hughes, 2000) (Figure 2.17). 


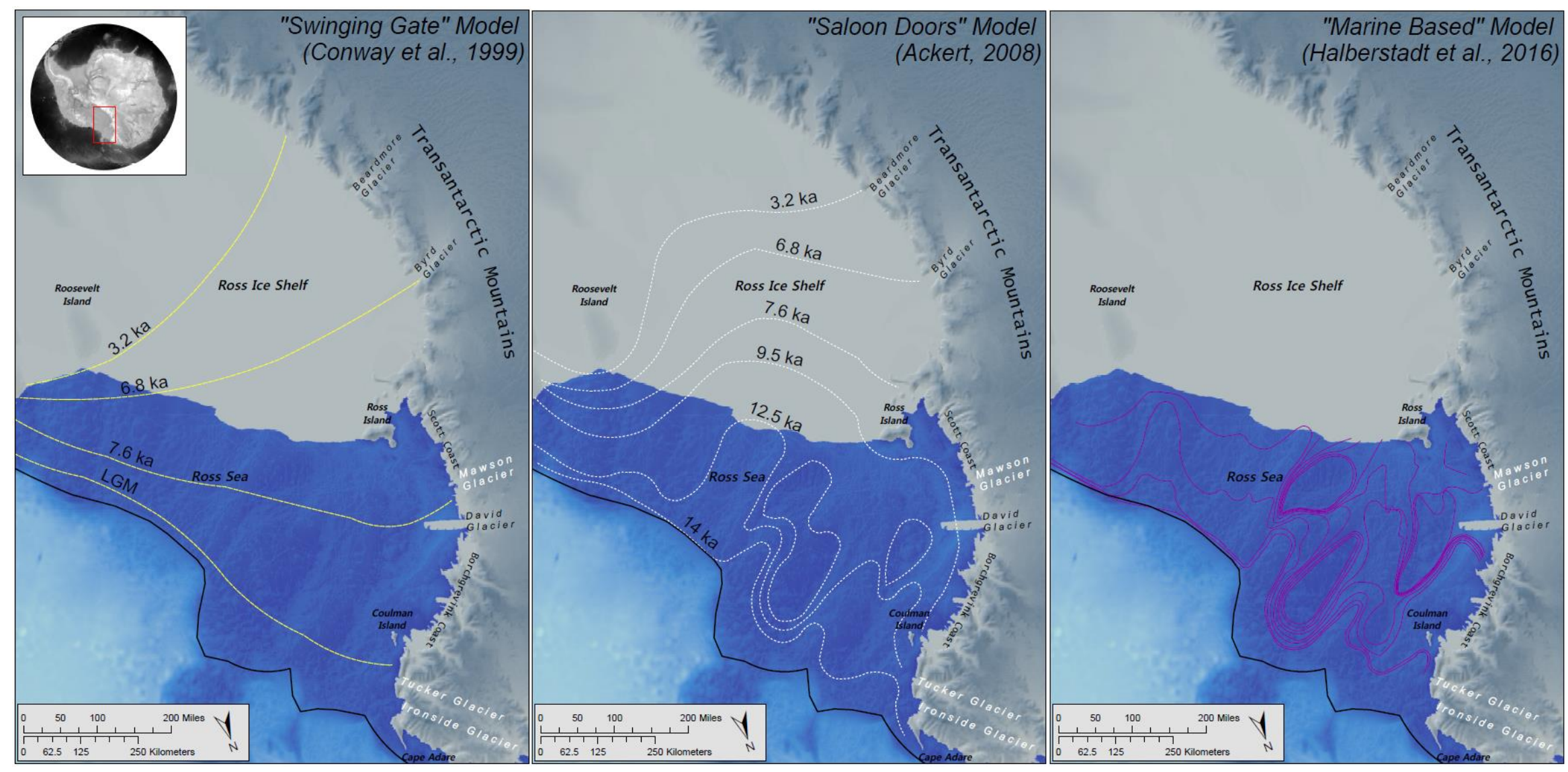

Figure 2.17 Grounded ice retreat hypotheses for the Ross Sea

The black line found in all maps is the maximum modelled extent of grounded ice during the Last Glacial Maximum (Denton and Hughes, 2000). The "Swinging Gate" hypothesis is shown with yellow lines, grounded ice is pinned at Roosevelt Island in the eastern Ross Sea and rapidly retreated south along the Victoria Land coast in the western Ross Sea (Conway et al., 1999). The "Saloon Doors" hypothesis is shown with white lines and is more complex. It starts with grounding line retreat in the central Ross Sea. After the initial phases of retreat the grounding line progresses radially to the east, south, and west (Ackert, 2008; Golledge, 2014). The Marine Based hypothesis involves the role of sea-floor topography as a key control on the rate and style of grounded ice retreat in the western Ross Sea (Halberstadt et al, 2016). 


\subsection{Background on erosion rates}

Earth's surface is continuously changing on geologic timescales, driven by tectonic activity, and changes in precipitation, temperature, and vegetation cover (Langbein and Schumm, 1958; Milliman and Syvitiski, 1992; Syvitiski, 2003; Collins, 2004; Montgomery, 2007; Ferrier et al., 2013; Hildy et al., 2014; Marshall et al., 2015; D'Arcy et al., 2017; Skov et al., 2019). Globally erosion rates calculated by terrestrial cosmogenic nuclides provide key information on long-term carbon sequestration via silicate weathering. These weathering rates are key to understanding long-term climate variability (Brener et al., 1983; Brenner, 1992). The highest erosion rates are typically in temperate climates with a mean annual temperature of $\sim 10 \mathrm{C}$ (Portenga et al., 2011). Both global and polar erosion rates are heavily dependent on rock type (Portenga et al., 2011; Marrero et al., 2018). The lowest erosion rates are found in arid climates regardless of temperature (Portenga et al.,2011). The global average bedrock erosion rate is $12 \pm 1.3 \mathrm{~m} / \mathrm{Myr}$ (Portenga et al., 2011) while the average polar bedrock erosion rate is 3.9 $\pm 0.39 \mathrm{~m} / \mathrm{Myr}$ (Portenga et al., 2011).

In polar climates, like Antarctica, erosion rates also provide critical information on the local glacial history and regional landscape evolution (Portenga et al., 2011; Marrero et al., 2018), as well as being necessary to calculate exposure ages (e.g. Fink et al., 2006). These three topics are addressed individually below.

Perhaps the most critical reason for knowing a local erosion rate for geochronology is to calculate exposure and burial ages. Due to the rapid attenuation of nuclide production in the Earth's surface even low erosion rates can have a substantial effect when calculating exposure ages. If the assumed erosion rate is too high or too low the sample age can be over or underestimated. Many terrestrial cosmogenic studies in Antarctica assume that no erosion has taken 
place since initial exposure. This will have little affect for some exposure age applications, for instance the rate, magnitude, or duration of ice surface elevation changes at outlet glaciers. However, including or excluding the erosion rate does affect the absolute timing of events. While the rate of erosion in Antarctica is very low by global standards, it is not zero and should be integrated into exposure age calculations.

Erosion rate studies also evaluate the landscape transiency and development through time. These types of studies are critical to understanding the life cycles of mountain ranges, the sedimentation cycle (Portenga et al., 2011) and the long-term, million-year, carbon cycle (Hilley and Porder, 2008).

Approximately $40 \%$ of the published Antarctic erosion rates are from the McMurdo Dry Valleys (Figure 2.18). The McMurdo Dry Valleys are renowned for extremely old deposits and primary surfaces (e.g. Brown et al., 1991; Brook et al., 1995; Ivy-Ochs et al., 1995). Much of the work in this area is done on the Sirius Group, a controversial moraine complex that is millions of years old (Brown et al., 1991; Brook et al., 1995; Ivy-Ochs et al., 1995; Bruno et al.,1997; Summerfield et al.,1999; Schäfer et al., 1999). Work in the McMurdo Dry Valleys is not representative of the environmental conditions found on nunataks fringing the Antarctic Ice Sheet at relatively lower latitudes where more maritime climates prevail. More work needs to be conducted outside of the McMurdo Dry Valleys and away from the hyperarid interior. 


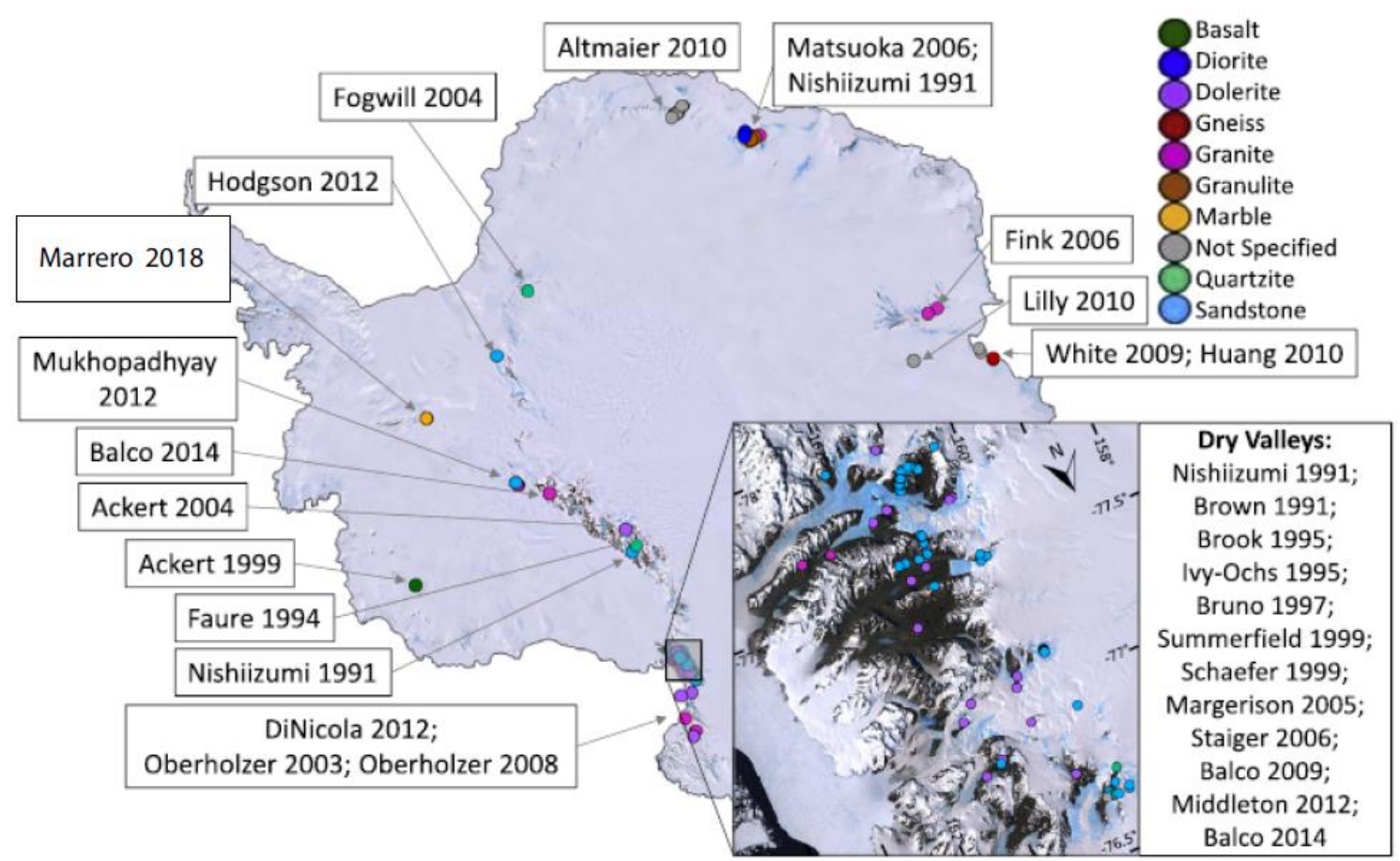

Figure 2.18 Locations of published erosion rate studies

This figure shows the geographical distribution and rock type for most of the previously published Antarctic erosion rate studies. There is a disproportionately large amount of work done in the McMurdo Dry Valleys on sandstone and dolerite (inset map). This figure is modified from Marrero et al, 2018.

\subsection{Research questions}

The previous sections have broadly introduced the topics covered in this thesis identifying research gaps. What follows are the research question which will help fill the research gaps and a thorough treatment of each topic.

1. Can a protocol be developed to improve quartz purification procedures for very-finegrained quartz rich rocks?

2. What is the magnitude, rate, and timing of ice-surface-elevation changes at Mawson and Tucker glaciers since the Last Glacial Maximum?

3. How does latitude, altitude, and continentality affect bedrock erosion rates in Antarctica?

4. 


\section{METHODS}

This section is intended as a specific guide to the methods required for each phase of work completed. For the detailed physical processing methods used refer to Appendix A and for the detailed chemical procedures applied refer to Appendix B.

\subsection{General physical and chemical processing stages}

The chemical methods employed in this research are slightly modified from pre-existing guidelines (von Blanckeburg et al., 1996; and von Blanckeburg et al., 2004; Norton et al., 2008, Jones et al., 2015). The purpose of this method is to isolate quartz from the rest of the sampled material and reduce the total volume of unnecessary and waste material. When samples have been separated to contain only quartz the chemical digestion stages can begin. Concentrated hydrofluoric acid is used to digest samples after a ${ }^{9} \mathrm{Be}$ carrier is added. Once total digestion has occurred two different ion-exchange resins allow for the elution of beryllium. The reclaimed beryllium is then precipitated as beryllium hydroxide and calcined. Once calcined it is mixed with niobium, packed into an AMS target, and sent for measurement at either Eidgenössische Technische Hochschule in Zurich, Switzerland or PRIME LAB in West Lafayette, Indiana USA.

\subsection{Cosmogenic nuclide sampling strategies for ice-surface reconstructions}

\subsubsection{Sampling approach for age-elevation profiles}

Glacial ice can completely inhibit particles of the secondary cascade from interacting with the lithosphere (Figure 3.1) therefore a rock surface below the glacier should not have a prior exposure history. This is advantageous because when that material is plucked from the bedrock and experiences sub-aerial exposure it will start accumulating nuclides for the first time. A 
diligently sampled elevation transect can reveal the past behaviour of an outlet glacier or ice stream in Antarctica.

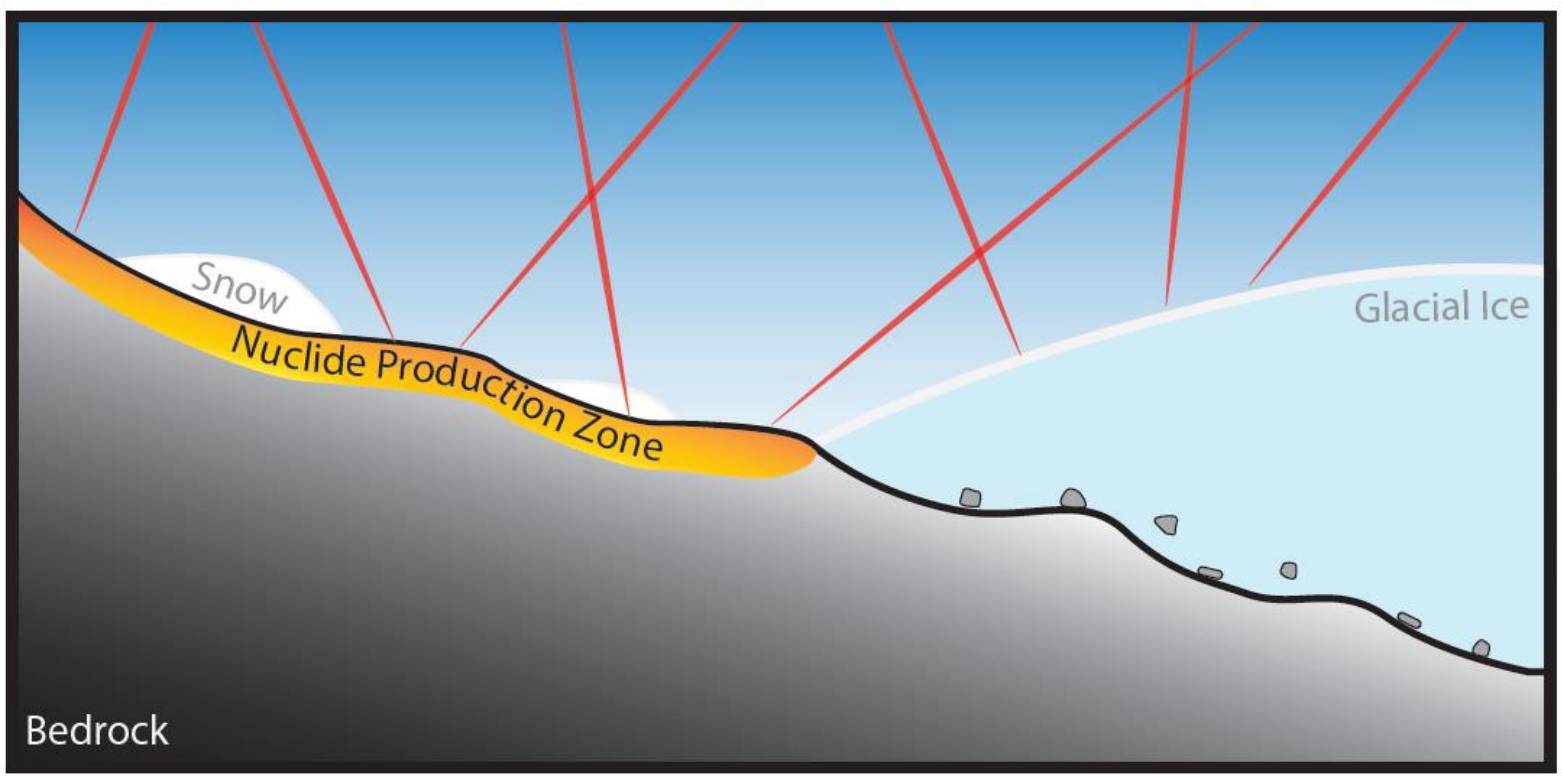

\section{Figure 3.1 Shielding by glacial ice}

Thick ice insulates the bedrock below a glacier from the cosmic ray flux. Snow also moderates the production of nuclides but may not totally shield the bedrock below from the cosmic ray flux.

Previous work provides examples of efficient and robust procedures to evaluate the thinning history of an outlet glacier in Antarctica (Gosse and Phillips, 2001; Stone et al., 2003; Sugden et al., 2005; Mackintosh et al. 2007; Balco, 2011; and Jones et al. 2015). The guidelines are based around paleo-glaciological and geological conditions present at the sample location and reduce the likelihood of collecting glacial erratics with an inherited cosmogenic signal.

Glacial erratics are collected on nuanataks adjacent to fast flowing portions of a glacier. Sample collection proceeds along elevation transects perpendicular to fast flowing ice near the outlet of a glacier (Figure 3.2). Ideally sample transects cover an elevation range of several hundred meters above the current ice surface (Figure 3.2). This methodology is routinely employed to explore the late Cenozoic to recent thinning history of outlet glaciers in Antarctica (e.g. Stone et al., 2003, Spector et al., 2017). Elevation transects provide a time-dependent 
exposure history for the glacier by dating the sediment deposited on nunataks as the glacier thins. Accordingly, glacially deposited sediment at higher elevations should produce relatively older ages then material deposited at lower elevations (Figure 3.2, Time 5). This strategy makes it possible to reconstruct the rate, timing, magnitude, and duration of ice-surfaceelevation changes for outlet glaciers. Reconstructing the ice surface elevation history of an outlet glacier provides both the means to evaluate and numerically model the centennial to millennial outlet glacier response. This method is colloquially known as the "Glacial Dipstick" and can illuminate both gradual and rapid surface elevation changes in Antarctica outlet glaciers and ice streams (Stone et al., 2003; Sugden et al., 2005; Mackintosh et al., 2007; Bromley et al., 2010; Todd et al., 2010; Jones et al., 2015; Balco et al., 2019; and Goehring et al., 2019).
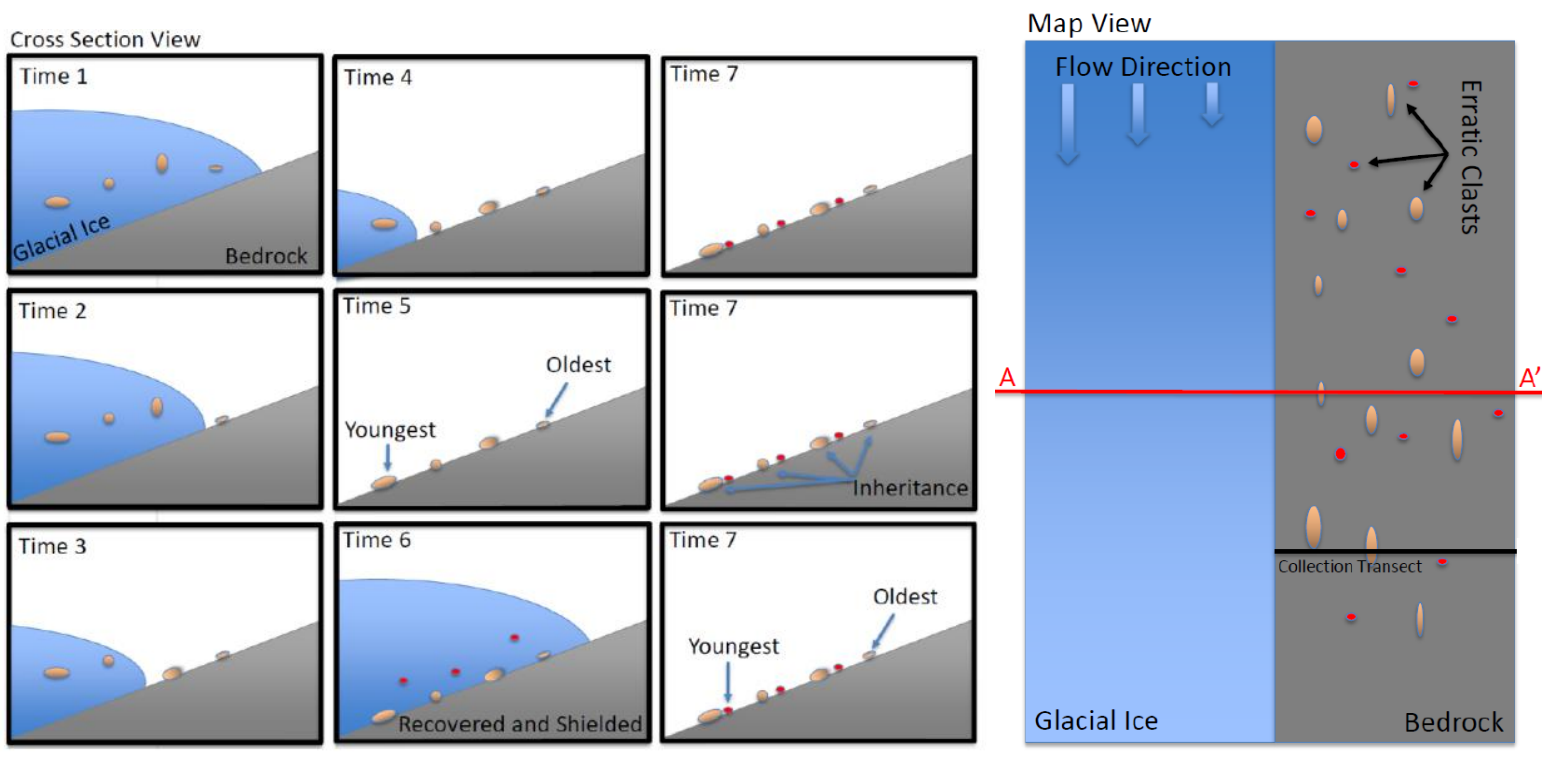

\section{Figure 3.2 Glacier dipstick approach}

This is a conceptual diagram explaining the Glacial Dipstick approach to sample collection and the role cold based ice plays in complicating a transect. Figure 3.2A is cross sectional view from (A-A' in map view) of a glacial margin broken into seven different time slices. In time 1 through time 5 the ice margin progressively retreats depositing sediment at each elevation as it retreats. In this simple system the highest erratic will have had the longest exposure history and the lowest erratic will have the shortest exposure history. In panels 6 and 7 a cold based glacier has moved over top of the previously deposited sediment and then retreated leaving a new accumulation of sediment with a different exposure history and complicating the previously simple vertical age relationship. It is worth noting that not all glaciers will be complicated by cold-based ice. Figure 3.2B is a map view of how samples are collected adjacent to the glacier. If samples can be collected in a perpendicular orientation to the glacier the age relationship illustrated in $3.2 \mathrm{~A}$ should hold true. 


\subsubsection{Glacial erratic sample selection criteria}

A rigorous sample selection criterion is outlined below. The criteria focus on the bedrock directly below a sample and clast specific evaluation. However, since cold-based ice cover can leave virtually no physical trace of its presence even strict sampling criteria cannot filter out all recycled material, therefore tens of samples are collected and processed for statistical analysis.

Samples are selected based on strict morphological and environmental criteria. The ideal sample is prominently and precariously positioned on glacially sculpted and striated bedrock. The rock type of the bedrock and glacial erratic should be obviously different. Morphologically the sample should be bulleted, striated, and faceted implying that the clast was plucked from bedrock under the glacier and transported through the zone of traction at the base of the glacier. The sample should have enough of the desired target mineral to provide a useful amount of sample after physical processing is complete. Glacial erratics sitting in areas of lower topography with lightly weathered bedrock are avoided due to the likelihood of intermittent or extended snow cover. Additionally, material sitting on fractured bedrock or till drapes are also avoided because of possible post-depositional movement.

\subsection{Site selection and site assessment}

This section explores why specific locations were selected for analysis. Site selection for an age elevation profile focusses on two large outlet glaciers, Mawson and Tucker glaciers, in Victoria Land Antarctica. Erosion rate site selection relies on location areas which have not been covered by ice and needs to be conducted in the field. 


\subsubsection{Age-elevation transect site selection}

Mawson Glacier was selected because it has not been previously studied and is a large outlet glacier draining $\sim 16,000 \mathrm{~km}^{2}$ of the East Antarctic Ice Sheet. There is also a strong possibility at this site to link terrestrial and marine records. Tucker Glacier was selected because it is near the hypothesised maximum ice extent in the western Ross Sea during the Last Glacial Maximum. The Tucker system will ideally provide control on the extent of grounded ice in the Western Ross Sea.

Initial site selection and assessment for Mawson Glacier was conducted using both glaciological mapping and glacier flow line modelling. The purpose of this type of analysis is to identify areas where warm-based erosive ice would have acted on bedrock to erode and abrade the surface and eventually, as the outlet glacier surface lowers, to deposit glacially transported sediment. As previously discussed, by targeting areas adjacent to modern, fastflowing ice the chance of collecting material with a prior exposure history, such as those shown in figure 3.2, is reduced. Several locations adjacent to Mawson Glacier were identified using this technique. These initial sites were further evaluated using vertical and oblique aerial photos to delineate locations with the highest sample retention potential.

Site assessment for Tucker Glacier consisted of a combination of Geographic Information System (GIS) analysis and airphoto interpretation. The components of the GIS analysis were a slope map, an aspect map, a geologic map of Victoria Land, topographic maps, and several topographic profiles. Many of these components were viewed in ArcSCENE yielding three dimensional renderings of the landscape and the desired map output (Figure 3.3). The second stage of site assessment involved collecting a large set of historic vertical and oblique imagery. Most of this imagery was collected by low-level and mid-level reconnaissance flights 
conducted by the United States Navy in the early 1960's. The historic imagery was compiled using the United States Geological Survey: Earth Explorer website. The oblique airphotos were useful for visualizing the potential sample locations with real imagery. Enough images were collected of each location to assess nunatak surface slopes from 360 degrees around the desired location. Finally, $50 \mathrm{~cm}$ resolution QuickBird imagery was purchased from DigitalGlobe for high-priority sites in and around Tucker Glacier. 


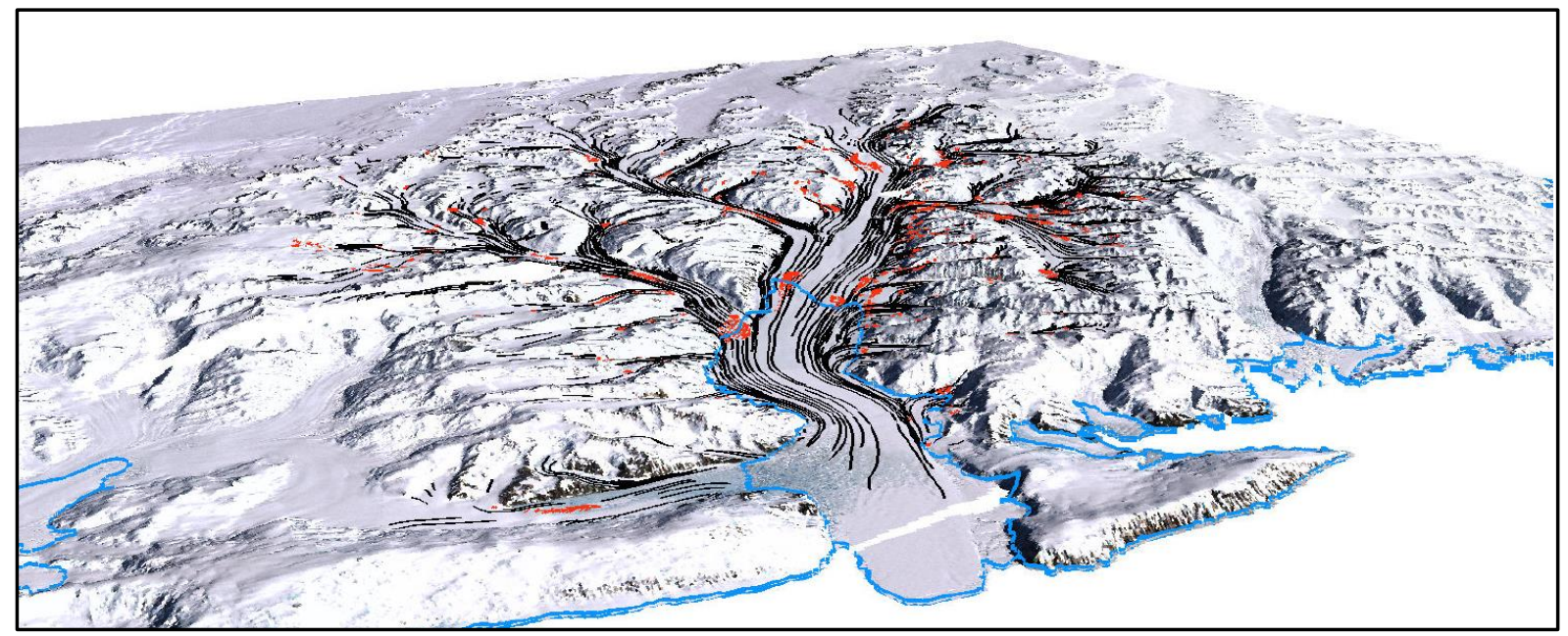

\section{Figure 3.3 ArcSCENE image of Tucker Glacier}

Oblique computer rendered image of Tucker Glacier. This type of analysis aided in site selection for the 2016/2017 summer season. The blue line is the position of the coastline and grounding line, black lines are mapped flow lineation showing the flow path of the glacier, and red lines are mapped crevasses which subtly indicate subglacial topography, stress localization on the ice, and changing flow dynamics. Image used LIMA satellite photography, REMA DEMs, and British Antarctic Survey coastline/grounding line data.

\subsubsection{Erosion rate site selection}

The criterion which needed to be met could only be evaluated on the ground and in real time.

The samples had to be from quartz rich bedrock, sufficiently high on a nunatak so that the site has ideally never been overtopped by glacial ice. Evidence used to assess if the site had been subject to glacial erosion include, a lack of glacial erratics, striated and polished surfaces, along with the presence of landforms and processes associated with periglacial environments. This evidence provides important clues to the glacial history of the location and helps ensure that a location is appropriate to sample collection.

\subsection{Calculating a linear thinning rate using IceTEA}

The MATLAB script IceTEA (Jones et al., 2019) was used to calculate glacial thinning histories. This approach uses randomly applied least squares linear regression to normally distributed age elevation data sets. A 10,000 iteration Monte Carlo simulation estimates the 
thinning rate from the total distribution of likely positive-sloping linear regressions. The regression analysis uses equation 3.1, below.

$$
y_{i}=\beta_{0}+\beta_{1} q_{i}
$$

(eq. 3.1)

The variable $y_{i}$ is the exposure age prediction for the position regressor $q_{i}$, the variable $\beta_{1}$ is the Pearson correlation coefficient for the mean age and position multiplied by the standard deviation of the mean ages divided by the standard deviation of the position, the variable $\beta_{0}$ is the mean observed age minus the mean sample position multiplied by the $\beta_{1}$ (Jones et al., 2019). 


\section{QUARTZ PURIFICATION: QUANTIFYING THE BUCKET}

\subsection{Introduction}

\section{$\underline{4.1 .1}$ Context}

The general sample purification guidelines are described in Kohl and Nishiizumi (1992) and Brown et al. (1991). These two studies form the foundation of chemical sample preparation for the terrestrial cosmogenic nuclide community. While these studies broadly outline the type of acids to use, the duration of sample purification for material, and the overall sample mass loss sufficient to remove meteoric ${ }^{10} \mathrm{Be}$, they do not explicitly address sample grain size below $500 \mu \mathrm{m}$ or the role major elements, ${ }^{9} \mathrm{Be}$, and ${ }^{10} \mathrm{~B}$ play in the purification process.

\section{$\underline{4.1 .2}$ Aim of work}

The aim of this work is two-fold: 1) provide a robust guideline for effectively removing elemental contaminants and meteoric ${ }^{10} \mathrm{Be}$ from $125-63 \mu \mathrm{m}$ sized samples, and 2) as a necessary step in order to process greywacke samples collected from Northern Victoria Land.

\subsection{Location and sample selection}

One sample, HP-16-11, was selected for detailed analysis because, it is typical of the $\sim 100$ samples collected during the Tucker Glacier field campaign. As such this study was required in order to analyse samples related to ice surface elevation changes during the Holocene at Tucker and Ironside glaciers in Antarctica.

\subsection{Method}

Standard quartz purification procedures are discussed in detail in section the "Quartz Purification Background". The salient points from that section are: $1{ }^{10} \mathrm{Be}$ is produced in both 
the atmosphere and in silicate minerals, 2) ${ }^{10} \mathrm{Be}$ is 1000 times more abundant in the atmosphere then in the upper $2 \mathrm{~m}$ of lithosphere, 3 ) meteoric ${ }^{10} \mathrm{Be}$ can adsorb to mineral grains and be held in oxide mineral overgrowths, 4) if a sample is not appropriately purified it can fail in chemistry or return an erroneous age, 5) it is imperative that major elements $(\mathrm{Al}, \mathrm{Ti}, \mathrm{Ca}, \mathrm{Na}$, $\mathrm{K}, \mathrm{Mn}, \mathrm{Mg}$, and $\mathrm{Fe}$ ) are removed before quartz is digested, and 6) a large $\mathrm{Ti}^{3+}$ and $\mathrm{Al}^{3+}$ cation load will suppress the ${ }^{9} \mathrm{Be}$ beam strength in an accelerator mass spectrometer (Hunt et al. 2008).

\section{$\underline{4.3 .1}$ Sample description}

Hand-sample HP-16-11 is a brownish-grey slightly weathered weakly-foliated fine-grained metasedimentary rock. The primary mineral grain size and composition is indistinguishable from the surrounding matrix. The foliation is weakly-developed and subparallel to the top and bottom surfaces of the boulder it was collected from. The glacial erratic itself is bulleted and faceted with locally developed fine pits on its surface and prior to collection was lying on a granite of the Admiralty Intrusive suite. The average thickness for the subsample is $4.3 \mathrm{~cm}$ and $6.8 \mathrm{~cm}$ from the apex of the clast. (Figure 4.1). 


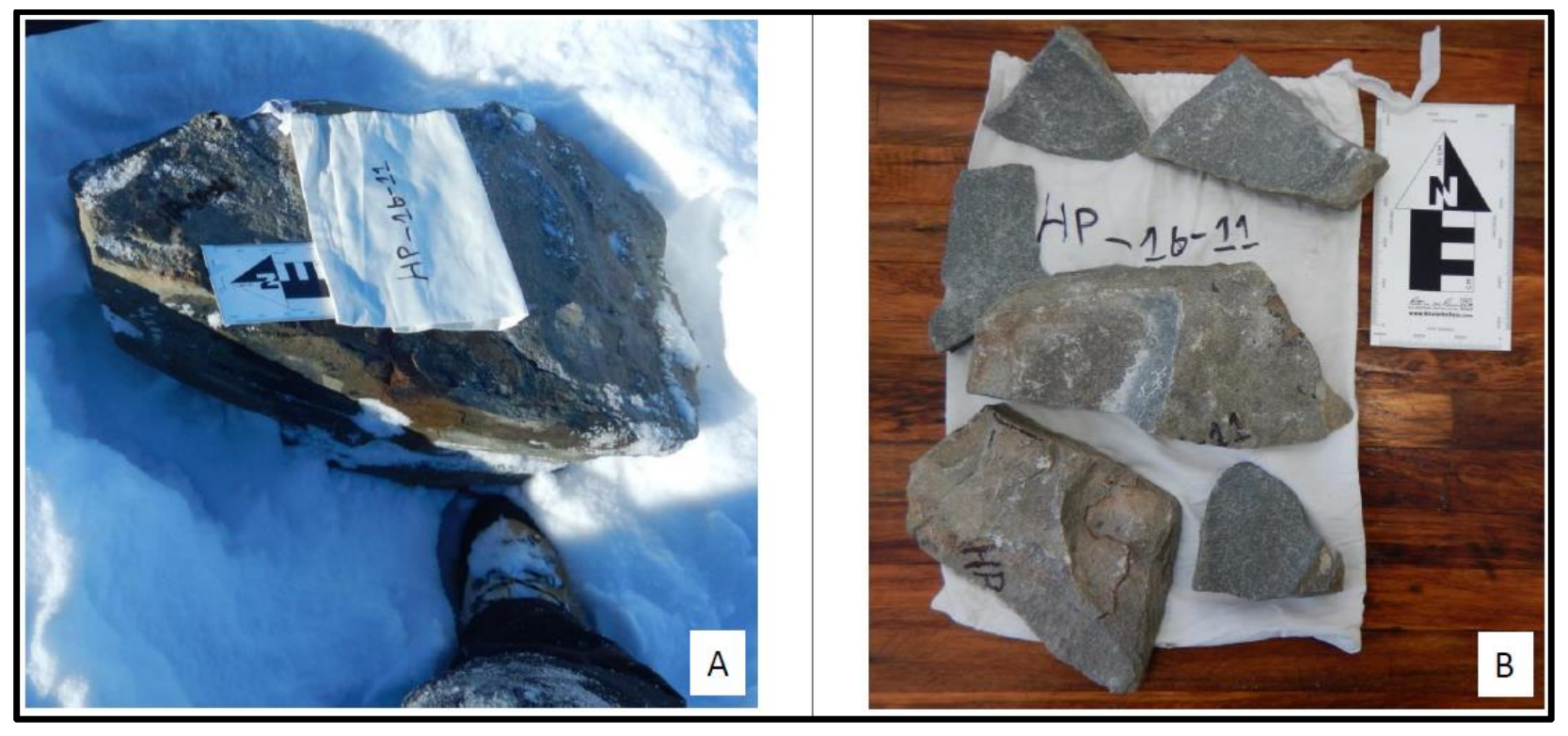

Figure 4.1 Hand sample in the field and in the lab

A) Field photo of sample collected at Helm Point in northern Victoria Land, Antarctica. B) The top $7 \mathrm{~cm}$ of the same glacial erratic photographed in A was sampled for terrestrial cosmogenic nuclide analysis.

In thin section the sample shows locally developed fused quartz with undulatory extinction and an average grain-size of $\sim 120 \mu \mathrm{m}$ and a maximum grain size of $1 \mathrm{~mm}$ in diameter. The matrix dominantly consists of phyllosilicate minerals (muscovite, sericite, and chlorite) with a few larger altered minerals grains (likely remnant feldspar grains). The thin section also shows pervasive very fine, opaque mineral inclusions dispersed throughout quartz grains (Figure 4.2). 


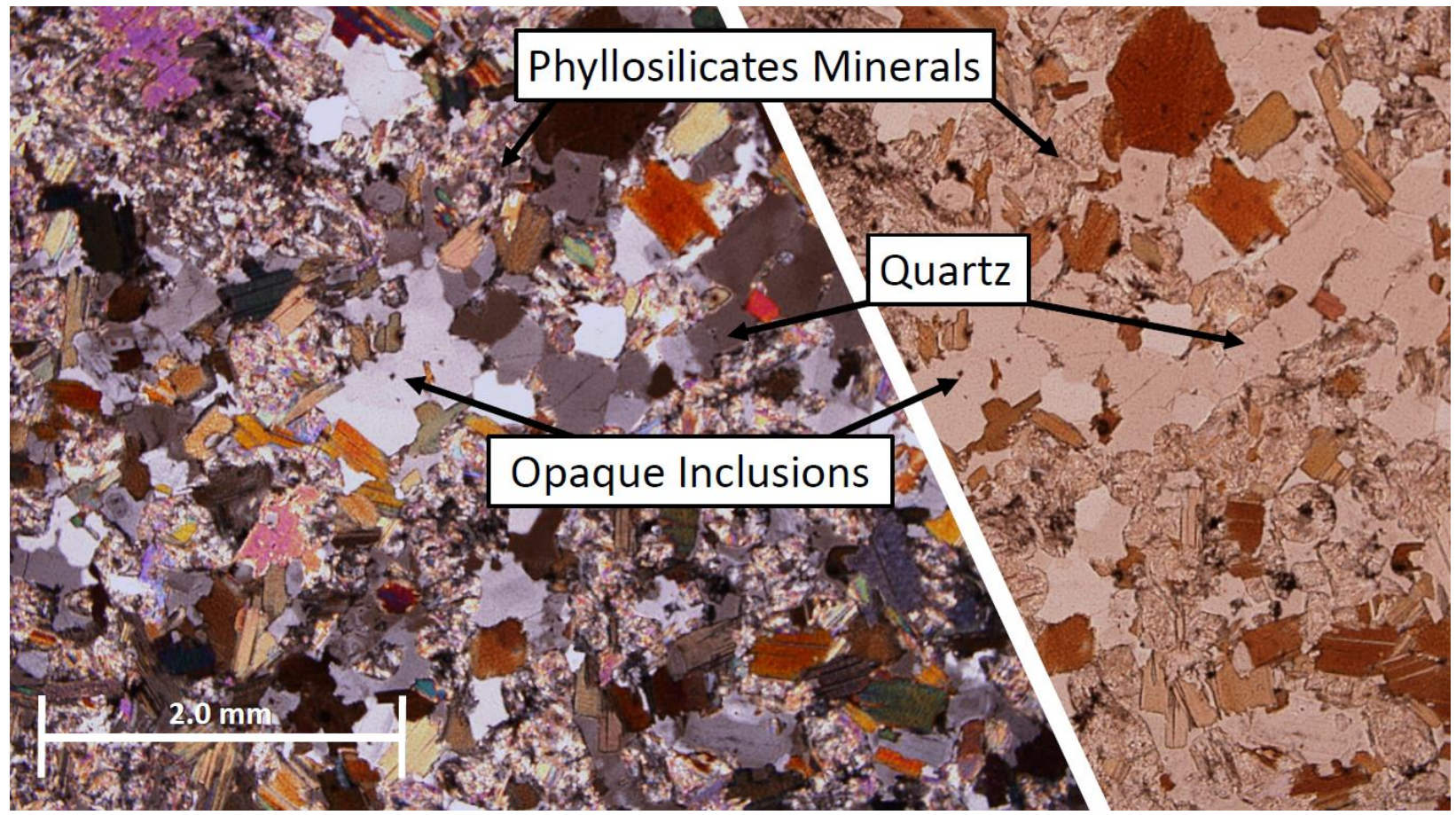

\section{Figure 4.2 Thin section of sample HP-16-11}

Thin section of sample Hp-16-11 in both plane polarized and cross polarized light. This figure shows the nature and texture of the rock as well as the size and character of the primary quartz grains.

\subsubsection{Physical processing}

The sample was physically processed by standard methods of cutting, crushing, and wet sieving in order to obtain the $125-63 \mu \mathrm{m}$ fraction of material. This was followed by magnetic mineral separation using a hand magnet and a Frantz mineral separator to separate the paramagnetic material from the non-paramagnetic material. The resulting mineral split was dominantly clean quartz with local opaque mineral inclusions and a thin veneer of opaque minerals coating some grains. The opaque mineral veneer is either an alteration or weathering rind. An alteration rind may incorporate meteoric ${ }^{10} \mathrm{Be}$ into its crystal lattice while a weathering rind may contain adsorbed ${ }^{10} \mathrm{Be}$. Removing the opaque mineral veneer is the first step in effectively removing all meteoric ${ }^{10} \mathrm{Be}$ from a sample (Figure 2.5). 


\subsection{Experimental}

\subsubsection{Method design and digestion procedure}

The method was designed to explore five different topics: 1) the feasibility of cleaning fine grained quartz, 2) in which chemical stage the majority of meteoric ${ }^{10} \mathrm{Be}$ is removed from a fine-grained sample, 3) what chemical stages remove significant amounts of major elements, 4) is percent mass loss a reasonable proxy for chemical purity, and 5) how cleaning changes the composition of material left in-situ in the quartz sample itself (Figure 4.3). By documenting each phase of this procedure, we quantify what elements are removed from a monomineralic quartz sample and what mass loss percentage is appropriate before digestion and further analysis is carried out.

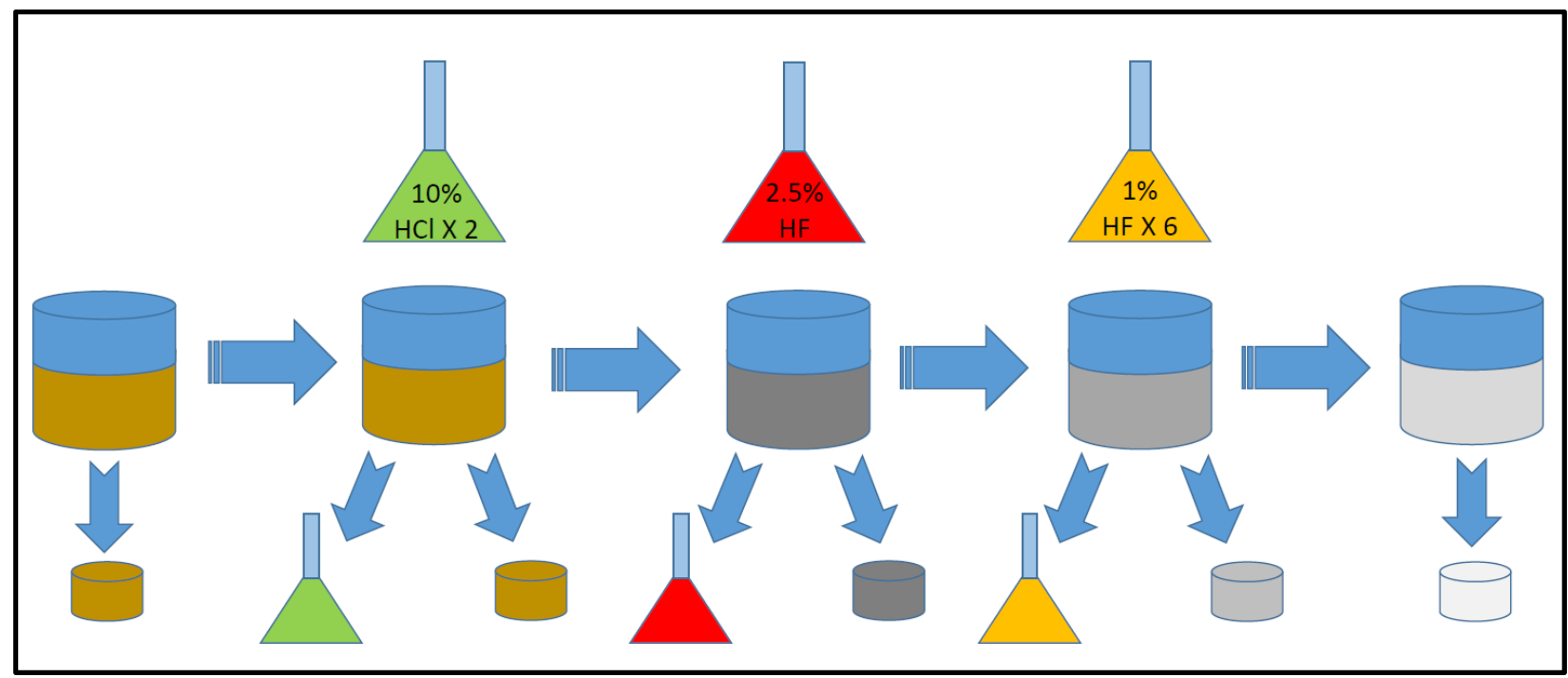

\section{Figure 4.3 Chemistry flowchart}

From left to right this flowchart illustrates the overall method design by which this experiment was conducted. We started with 35 grams of untreated quartz, added two rounds of $10 \% \mathrm{HCl}$ and collected the supernate, and a one-gram subsample. Then we treated the sample with one round of $2.5 \% \mathrm{HF}$ and collected the supernate and a one-gram subsample. Finally, we treated the quartz with six rounds of $1 \% \mathrm{HF}$ and collected the supernate and a one-gram subsample from each round of chemistry. We did this to document the transition from the initial to the final sample in both the sample and the leachate. This type of time series constrains when and what type of material is being removed from the primary sample. 


\section{$\underline{4.4 .2} \underline{\text { Initial setup-progress }}$}

The initial sample mass was 40-grams of magnetically separated monomineralic quartz. The total sample mass was split into a 5-gram untreated sample and a 35-gram treatment sample. Both were placed in clean $350 \mathrm{ml}$ Savillex beakers. The 5-gram sample was immediately digested in $50 \% \mathrm{HF}$ and set aside for later analysis. The 35-gram sample was treated twice with $200 \mathrm{ml}$ of $10 \% \mathrm{HCl}$ for $\sim 48$ hours each, once with $200 \mathrm{ml}$ of $2.5 \% \mathrm{HF}$ and $1 \% \mathrm{NHO}_{3}$ for $\sim 24$ hours, and six times with $200 \mathrm{ml}$ of $1 \%$ HF for $\sim 24$ hours each (Table 4.1). All samples were heated at $60^{\circ} \mathrm{C}$ and agitated by hand every hour for the first $6-8$ hours of heating after each acid change. At each acid stage the used supernate was decanted into a new clean Savillex Teflon beaker and saved for ICP-MS analysis. Before adding the next acid stage, the sample was rinsed three to four times with enough $18 \Omega$ Mili-Q water to cover the sample with an excess of $0.5 \mathrm{~cm}$ of fluid over the sample. During the rinse process the samples were let to settle away from heat for 10-20 minutes, to fluid clarity, in order to avoid decanting finegrained material. The rinse water was not retained for analysis and was decanted into a waste container. After rinsing the sample, it was fully dried, massed, and a one-gram subsample was collected for photo documentation, digestion, and subsequent ICP-MS analysis. This process was repeated for every step of the cleaning procedure (Figure 4.3).

\section{$\underline{4.4 .3}$ Procedures for inductively coupled plasma mass spectrometer analysis}

The supernates were dried at $100^{\circ} \mathrm{C}$ and produced a variety of residual material. The dried $\mathrm{HF}$ supernates produced a white cake of material, while the $\mathrm{HCl}$ supernates left a thick red-orange waxy residue in the beaker. Hydrochloric acid (6M) was added to all samples in order to break each respective residue down for ICP-MS analysis (Figure 4.4). Each supernate required a variable amount of $6 \mathrm{M} \mathrm{HCl}$ to totally dissolve the fluoride cake (Tables 4.2-4.6). After the initial fluoride cake was redissolved the sample was dried down and taken up in variable 
amounts of $9 \mathrm{M} \mathrm{HNO}_{3}$ (Tables 4.2-4.6). At this stage three samples were heated to $100^{\circ} \mathrm{C}$ for 10 days to fully break down the dried residue $\left(1^{\text {st }} 10 \% \mathrm{HCl}, 2.5 \% \mathrm{HF}\right.$ and the $1^{\text {st }} 1 \% \mathrm{HF}$ supernates). Once fully dissolved in $9 \mathrm{M} \mathrm{HNO}_{3}$ a $1 \mathrm{ml}$ aliquot of each sample was taken after the sample was totally dissolved and a two-stage 10,000-fold dilution was completed to prepare the samples for analysis. The dilution was done using $1 \mathrm{M}$ high-purity distilled $\mathrm{HNO}_{3}$ (Tables 4.2-4.6).

The one-gram quartz subsamples were photographed and digested in 50\% concentrated HF according to a standard 4:1 HF to sample ratio for closed beaker digestion. The resulting white fluoride cake was broken in $10 \mathrm{ml}$ of $6 \mathrm{M} \mathrm{HCl}$, dried again, and redissolved in $10 \mathrm{ml} 9 \mathrm{M} \mathrm{HNO}_{3}$ (Figure 4.4). A $1 \mathrm{ml}$ aliquot of each sample was collected, and a two stage 10,000-fold dilution was completed to prepare the sample for analysis. This dilution was also completed using $1 \mathrm{M}$ high-purity distilled $\mathrm{HNO}_{3}$ (Table 4.4). 


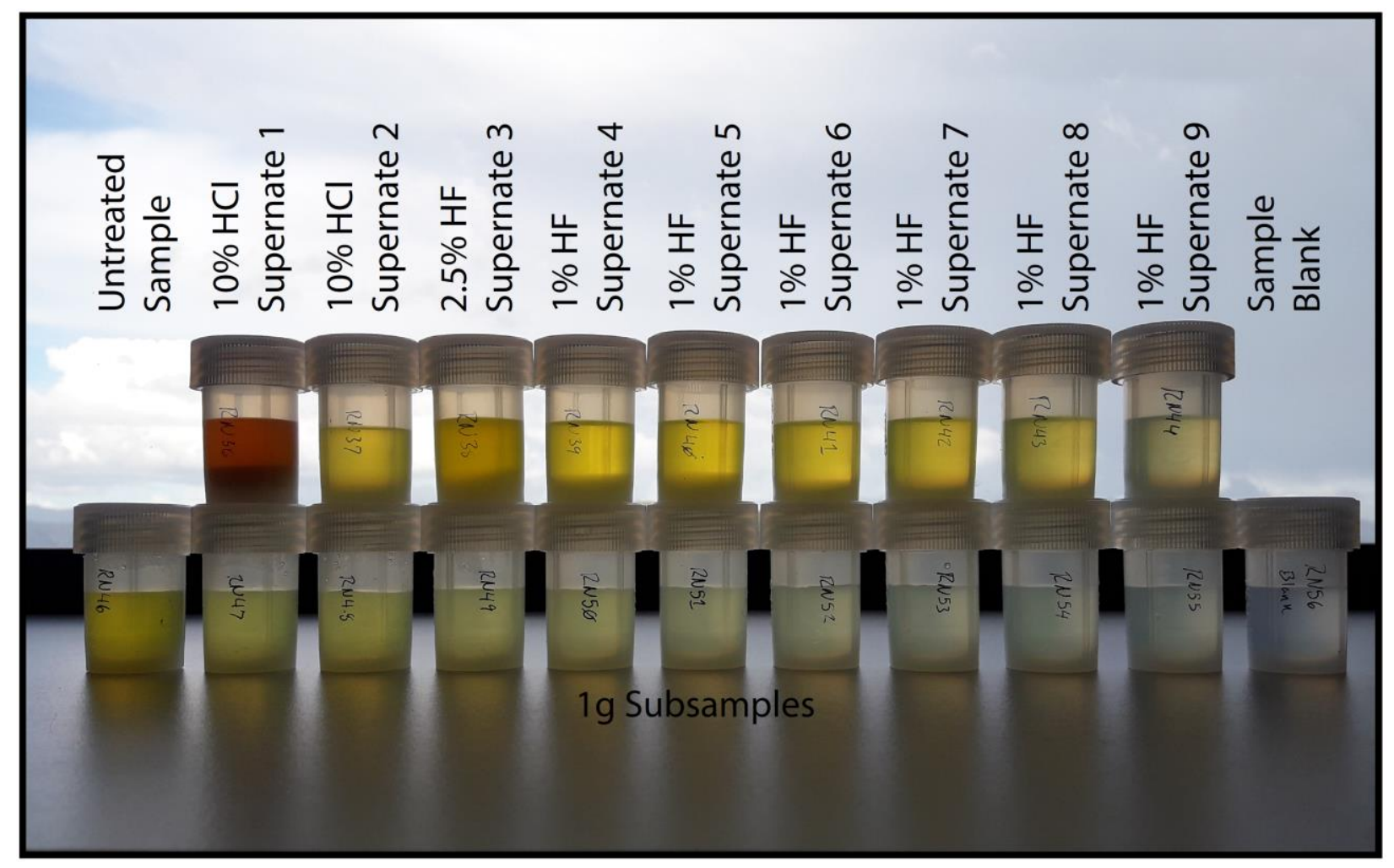

Figure 4.4 Subsamples and supernates in solution

This photo shows each sample both supernate and post digestion one-gram subsample in $10 \mathrm{ml}$ of $6 \mathrm{M} \mathrm{HCl}$ during the initial salt breaking stage. Note the sharp contrast as the sample purification procedure progresses from left to right. The supernates along the top have clearly stripped a large amount of material from the total sample. While the one-gram subsamples are progressively approaching the clarity of the blank seen at the end.

All sample dilutions were measured in the Geochemistry Lab at Victoria University of

Wellington, on a Thermo-Fisher Element 2 single-collector, double-focusing magnetic sectorfield ICP-MS with mg/L to $\mathrm{pg} / \mathrm{L}$ sensitivity. Major element analyses were standardized against synthetic multi-element standards at concentrations of $0.1 \mathrm{ppb}, 0.2 \mathrm{ppb}, 1 \mathrm{ppb}, 2 \mathrm{ppb}$, and 4 ppb. Major element analysis was conducted at medium and high resolution to reduce isobaric interference of other compounds within similar mass ranges. Beryllium-9 and Boron-10 analyses were standardised using a synthetic standard at concentrations of $0.2 \mathrm{ppb}, 0.5 \mathrm{ppb}, 1$ ppb, $2 \mathrm{ppb}$, and $4 \mathrm{ppb}$ and measured at high resolution to reduce isobaric interference. 


\subsubsection{Blank and standard scheme}

A comprehensive multiphase blank and standard scheme was developed to convert counts per second (CPS) to concentrations and to constrain the amount of material inadvertently added to the samples at each stage of the experiment. This scheme is composed of three different stages: 1) the ICP-MS standards for 10 elements, 2) a set of fluoride salt decomposition blanks, and 3) a set of digestion process blanks. All samples were corrected with the appropriate standard and blanks for their chemical phase. All sample concentrations were calculated from these standards using Microsoft Excel.

The salt decomposition blank correction set was made to correct for various amounts of $6 \mathrm{M}$ $\mathrm{HCl}$ and $9 \mathrm{M} \mathrm{HNO}_{3}$ used to break fluoride salts produced as a result of the dry down procedure (10 $\mathrm{ml}, 35 \mathrm{ml}$, and $122.5 \mathrm{ml}$ ). This blank set was only used to correct the dry supernate and the post digestion one-gram subsamples. It was not used to correct the process blanks as the process blanks did not require additional acid to break a precipitate salt.

The process blank correction set was made to correct for different phases of the primary experiment. A blank was created for each transition in the chemistry and underwent the same treatment as each sample. The four process blanks represent the $\mathrm{HCl}$ phase, $2.5 \% \mathrm{HF}$ phase, $1^{\text {st }} 1 \%$ HF phase, and the $6^{\text {th }} 1 \%$ HF phase. This blank set was used to correct only the dry supernates and the post digestion one-gram subsamples. It was not used to correct the salt decomposition blanks.

\subsubsection{Meteoric ${ }^{10} \mathrm{Be}$ removal}

The purpose of this portion of the experiment is to determine which acid phase removes the

majority of the meteoric ${ }^{10} \mathrm{Be}$. The supernatant material and the final purified sample were analysed to quantify the amount of meteoric ${ }^{10} \mathrm{Be}$ leached from the samples. 
Preparation for this phase involved combining individual supernate acid stages together. The $10 \% \mathrm{HCl}$ stages were combined with one another, the $1^{\text {st }}$ and $2^{\text {nd }} 1 \% \mathrm{HF}$ stages were combined, the $3^{\text {rd }}$ and $4^{\text {th }} 1 \%$ HF stages were combined, and $5^{\text {th }}$ and $6^{\text {th }} 1 \%$ HF stages were combined. The only acid stage analysed as an individual was the $2.5 \% \mathrm{HF}$ (acid stage 3 ). The supernates were then heated at $100^{\circ} \mathrm{C}$ until dry. Once dry, $0.8 \mathrm{ml}$ of a $305 \mathrm{ppm}{ }^{9} \mathrm{Be}$ carrier was added to each sample and run through a set of anion (2 ml of Biorad AG1-X8 100-200 mesh resin in a $15 \mathrm{ml}$ Eichron column) and cation (5 ml of Biorad AG50-X8 200-400 mesh resin in a $7.5 \mathrm{ml}$ column) columns (von Blanckeburg et al., 1996; and von Blanckeburg et al., 2004; Norton et al., 2008, Jones et al., 2015). The samples were then calcined, mixed with $\mathrm{Nb}$ flux, and packed into targets. Sample analysis was conducted on an upgraded FN 7 MV tandem Accelerator Mass Spectrometer (AMS) at PRIME Lab in Purdue University, United States.

\subsection{Results}

\subsubsection{Total mass loss throughout the purification process}

Loss of sample mass is an easy, economical, and straight-forward method to determine the cleanliness of a monomineralic quartz sample, provided there is no material lost while decanting (Figure 4.5 and Table 4.1). At each phase of chemistry, the sample was massed, and a one-gram subsample was collected. Samples were carefully decanted so-as-to not lose any quartz from the beakers. No obvious decanting errors occurred while collecting these data.

The initial $\mathrm{HCl}$ phase (acid stage 1) has a $\sim 4 \%$ drop in mass relative to $\sim 1.4$ grams of material being digested. This phase is responsible for the removal of oxide forming elements (Fe and $\mathrm{Mn})$, carbonate forming elements $(\mathrm{Ca}$ and $\mathrm{Mg}$ ), and phyllosilicates $(\mathrm{Al}$ and $\mathrm{K})$. The second $\mathrm{HCl}$ phase (acid stage 2) shows that $\sim 93 \%$ of the oxide and carbonate forming elements are removed in the initial $\mathrm{HCl}$ phase (Figure 4.5 and Table 4.1). Preforming two $\mathrm{HCl}$ leach cycles 
ensures a reduced ion load from Alkali metals held in solution during column chemistry (Blanckenburg et al., 1996).

The single $2.5 \%$ HF stage (acid stage 3 ) removed $\sim 3.9$ grams of sample and is the largest single contributor to mass loss in this procedure. The six 1\% HF phases (acid stage 4-9) remove 6.7 grams of material and account for $\sim 20 \%$ of the total mass loss required for purification (Figure 4.5 and Table 4.1). The HF series focusses on the removal of $\mathrm{Al}, \mathrm{Ti}, \mathrm{Na}$, and $\mathrm{K}$ likely from mineral overgrowths and the quartz crystal itself.

Removal of significant amounts of $\mathrm{Al}^{3+}$ and $\mathrm{Ti}^{3+}$ is important because high concentrations of these trivalent ions can overwhelm the ion exchange resins used to elute Be (Blankenburg et al, 1996) and suppress ion beam current in an accelerator mass spectrometer (Hunt et al., 2008). Typically, oxalic acid is used to reduce the interaction of trivalent ions (e.g. $\mathrm{Al}^{3+}, \mathrm{Fe}^{3+}$, and $\mathrm{Ti}^{3+}$ ) with the cation columns (Von Blanckenburg et al., 1996; Hunt et al., 2008). 


\section{Table 4.1 Timeseries of mass loss}

This table shows the total and cumulative mass loss of sample HP-16-11 from the beginning of the sample to completion and the amount of material removed from the sample for analysis throughout the purification process.

Total mass loss

\begin{tabular}{|c|c|c|c|c|c|c|c|c|c|c|c|}
\hline Acid Type & $\begin{array}{l}\text { Initial } \\
\text { Mass }\end{array}$ & $\begin{array}{c}10 \% \mathrm{HCl} \\
\# 1\end{array}$ & $\begin{array}{c}10 \% \mathrm{HCl} \\
\# 2 \\
\end{array}$ & $\begin{array}{c}2.5 \% \mathrm{HF} \\
\# 1\end{array}$ & $\begin{array}{c}1 \% \mathrm{HF} \\
\# 2 \\
\end{array}$ & $\begin{array}{c}1 \% \mathrm{HF} \\
\# 3 \\
\end{array}$ & $\begin{array}{c}1 \% \mathrm{HF} \\
\# 4\end{array}$ & $\begin{array}{c}1 \% \mathrm{HF} \\
\# 5 \\
\end{array}$ & $\begin{array}{c}1 \% \mathrm{HF} \\
\# 6 \\
\end{array}$ & $\begin{array}{c}1 \% \mathrm{HF} \\
\# 7 \\
\end{array}$ & $\begin{array}{c}\text { Total } \\
\text { Mass Loss }\end{array}$ \\
\hline Acid Stage & 0 & 1 & 2 & 3 & 4 & 5 & 6 & 7 & 8 & 9 & \\
\hline Initial Mass (g) & 35.0685 & 188.2938 & 187.238 & 182.3065 & 180.104 & 177.7682 & 175.63 & 173.64 & 171.723 & 169.886 & \\
\hline After $1 \mathrm{~g}$ Removal & 189.7146 & 187.348 & 186.1883 & 181.4276 & 179.108 & 176.7849 & 174.715 & 172.748 & 170.678 & 168.835 & \\
\hline Sample Removal (g) & 0 & 0.9458 & 1.0497 & 0.8789 & 0.9951 & 0.9833 & 0.9154 & 0.8916 & 1.0452 & 1.0517 & 8.7567 \\
\hline Sample Digestion (g) & 0 & 1.4208 & 0.11 & 3.8818 & 1.3241 & 1.3402 & 1.1547 & 1.0751 & 1.0251 & 0.7915 & 12.1233 \\
\hline Percent Digestion & 0.00 & 4.05 & 0.31 & 11.07 & 3.78 & 3.82 & 3.29 & 3.07 & 2.92 & 2.26 & $34.57 \%$ \\
\hline
\end{tabular}

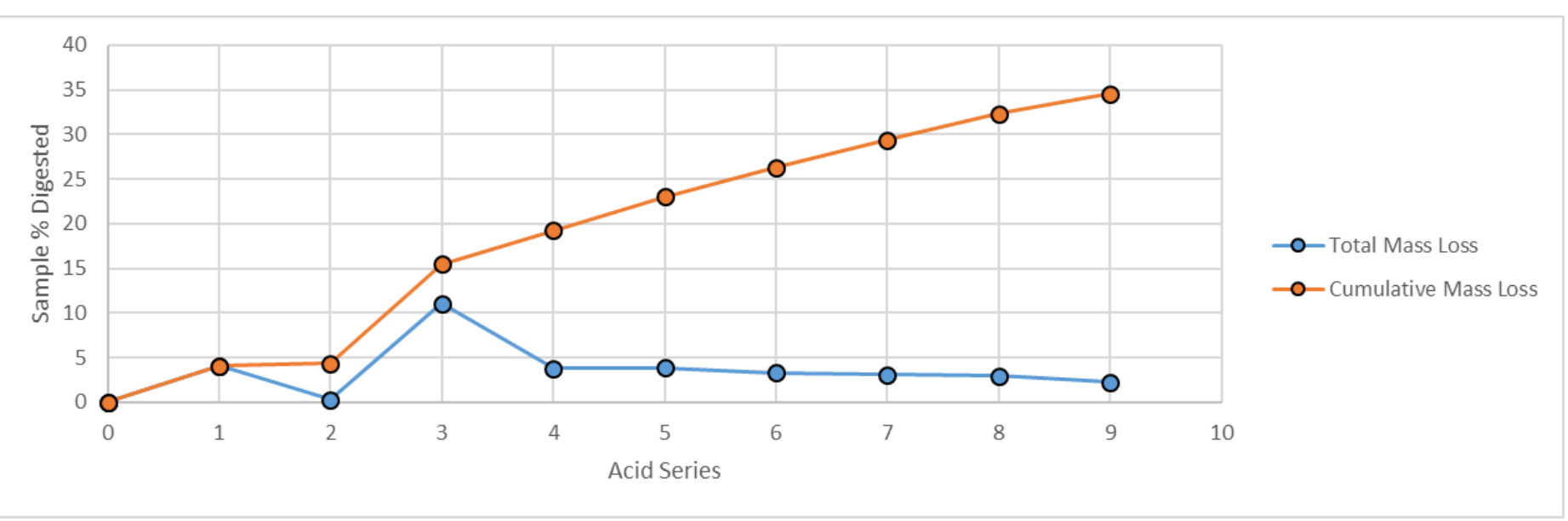

\section{Figure 4.5 Timeseries of mass loss}

This graph shows the total and cumulative mass loss for the sample HP-16-11 as it progressed through quartz purification. It also shows the mass of material removed at each stage. 


\subsubsection{Fine-grained sample progression through chemistry}

This section documents the successful progression of fine-grained quartz (125 $\mu \mathrm{m}-63 \mu \mathrm{m})$ through the purification process. Each section is separated into chemical phases, which are defined by the acid used. This is intended as a visual guide for the general purification process. The effect of chemistry on grain size and any visibly observed changes to the sample throughout the process is the primary focus of this section (Tables 4.2-4.5). 


\subsubsection{Purification element data}

\section{Table 4.2 Acid supernate timeseries (ppm)}

This table contains the timeseries of each isotope tested in the supernate removed from the samples. Each sample was collected by decanting the supernate into a clean Savallex Teflon beaker. The data are presented in parts per million.

\begin{tabular}{|c|c|c|c|c|c|c|c|c|c|}
\hline $\begin{array}{c}\text { Acid } \\
\text { Supernate } \\
\text { (ppm) }\end{array}$ & & & & & & & & & \\
\hline $\begin{array}{l}\text { Sample } \\
\text { Name }\end{array}$ & RN36 & RN37 & RN38 & RN39 & RN40 & RN41 & RN42 & RN43 & RN44 \\
\hline Acid Type & $\begin{array}{c}10 \% \mathrm{HCl} \\
\# 1\end{array}$ & $\begin{array}{c}10 \% \mathrm{HCl} \\
\# 2 \\
\end{array}$ & $\begin{array}{c}2.5 \% \mathrm{HF} \\
\# 1\end{array}$ & $\begin{array}{c}1 \% \mathrm{HF} \\
\# 2\end{array}$ & $\begin{array}{c}1 \% \mathrm{HF} \\
\# 3\end{array}$ & $\begin{array}{c}1 \% \mathrm{HF} \\
\# 4\end{array}$ & $\begin{array}{c}1 \% \mathrm{HF} \\
\# 5\end{array}$ & $\begin{array}{c}1 \% \mathrm{HF} \\
\# 6\end{array}$ & $\begin{array}{c}1 \% \mathrm{HF} \\
\# 7\end{array}$ \\
\hline Acid Stage & 1 & 2 & 3 & 4 & 5 & 6 & 7 & 8 & 9 \\
\hline${ }^{9} \mathrm{Be}$ & $3.94 \mathrm{E}-01$ & $1.24 \mathrm{E}-01$ & 8.77E-01 & $9.87 \mathrm{E}-01$ & $8.30 \mathrm{E}-01$ & 6.04E-01 & 4.72E-01 & 3.73E-01 & $8.87 \mathrm{E}-02$ \\
\hline B & $1.65 \mathrm{E}+00$ & $8.68 \mathrm{E}-01$ & $5.03 \mathrm{E}+00$ & $4.22 \mathrm{E}+00$ & $3.01 \mathrm{E}+00$ & $2.37 \mathrm{E}+00$ & $1.63 \mathrm{E}-02$ & $1.82 \mathrm{E}+00$ & $6.25 \mathrm{E}-02$ \\
\hline $\mathrm{Fe}$ & $1.85 \mathrm{E}+05$ & $4.44 \mathrm{E}+03$ & $8.24 \mathrm{E}+03$ & $7.04 \mathrm{E}+03$ & $6.30 \mathrm{E}+03$ & $4.68 \mathrm{E}+03$ & $3.43 \mathrm{E}+03$ & $1.33 \mathrm{E}+03$ & $4.32 \mathrm{E}+02$ \\
\hline $\mathrm{Mn}$ & $2.83 \mathrm{E}+03$ & $1.38 \mathrm{E}+01$ & $2.32 \mathrm{E}+01$ & $2.23 \mathrm{E}+01$ & $1.91 \mathrm{E}+01$ & $1.51 \mathrm{E}+01$ & $1.13 \mathrm{E}+01$ & $4.16 \mathrm{E}+00$ & $1.11 \mathrm{E}+00$ \\
\hline $\mathrm{Ca}$ & $2.47 \mathrm{E}+05$ & $1.76 \mathrm{E}+02$ & $1.69 \mathrm{E}+03$ & $1.12 \mathrm{E}+03$ & $9.12 \mathrm{E}+02$ & $1.02 \mathrm{E}+03$ & $7.14 \mathrm{E}+02$ & $1.28 \mathrm{E}+02$ & $1.45 \mathrm{E}+01$ \\
\hline $\mathrm{Mg}$ & $7.78 \mathrm{E}+04$ & $1.92 \mathrm{E}+03$ & $2.53 \mathrm{E}+03$ & $1.04 \mathrm{E}+03$ & $1.17 \mathrm{E}+03$ & $2.35 \mathrm{E}+03$ & $2.39 \mathrm{E}+03$ & $8.02 \mathrm{E}+02$ & $2.54 \mathrm{E}+02$ \\
\hline $\mathrm{Al}$ & $9.43 E+04$ & $7.94 \mathrm{E}+03$ & $1.00 \mathrm{E}+05$ & $1.12 \mathrm{E}+05$ & $1.07 \mathrm{E}+05$ & $1.17 \mathrm{E}+05$ & $9.24 \mathrm{E}+04$ & $2.30 \mathrm{E}+04$ & $5.25 \mathrm{E}+03$ \\
\hline $\mathrm{Ti}$ & $9.89 \mathrm{E}+01$ & $2.45 \mathrm{E}+02$ & $1.48 \mathrm{E}+03$ & $1.55 \mathrm{E}+03$ & $1.32 \mathrm{E}+03$ & $9.74 \mathrm{E}+02$ & $7.44 \mathrm{E}+02$ & $2.92 \mathrm{E}+02$ & $9.40 \mathrm{E}+01$ \\
\hline $\mathrm{Na}$ & $2.14 \mathrm{E}+03$ & $4.81 \mathrm{E}+02$ & $1.01 \mathrm{E}+05$ & $9.16 \mathrm{E}+04$ & $6.87 \mathrm{E}+04$ & $6.23 \mathrm{E}+04$ & $4.66 \mathrm{E}+04$ & $8.18 \mathrm{E}+03$ & $1.35 \mathrm{E}+03$ \\
\hline $\mathrm{K}$ & $1.09 \mathrm{E}+04$ & $3.59 \mathrm{E}+03$ & $2.75 \mathrm{E}+04$ & $2.59 \mathrm{E}+04$ & $2.28 \mathrm{E}+04$ & $1.75 \mathrm{E}+04$ & $1.34 \mathrm{E}+04$ & $3.58 \mathrm{E}+03$ & $1.44 \mathrm{E}+03$ \\
\hline
\end{tabular}




\section{Table 4.3 Acid supernate timeseries $(\mu \mathrm{g})$}

This table contains the timeseries of each isotope tested in the supernate removed from the samples. Each sample was collected by decanting the supernate into a clean Savallex Teflon beaker. The data are presented in micrograms and are graphed below in figures 4.7-4.10 and 4.12-4.17.

\section{Acid}

\section{Supernate}

in

Micrograms

\begin{tabular}{|cccccccccc|}
\hline $\begin{array}{c}\text { Sample } \\
\text { Name }\end{array}$ & RN36 & RN37 & RN38 & RN39 & RN40 & RN41 & RN42 & RN43 & RN44 \\
\hline \hline Acid Type & $\begin{array}{c}10 \% \mathrm{HCl} \\
\# 1\end{array}$ & $\begin{array}{c}10 \% \mathrm{HCl} \\
\# 2\end{array}$ & $\begin{array}{c}2.5 \% \mathrm{HF} \\
\# 1\end{array}$ & $\begin{array}{c}1 \% \mathrm{HF} \\
\# 2\end{array}$ & $\begin{array}{c}1 \% \mathrm{HF} \\
\# 3\end{array}$ & $\begin{array}{c}1 \% \mathrm{HF} \\
\# 4\end{array}$ & $\begin{array}{c}1 \% \mathrm{HF} \\
\# 5\end{array}$ & $\begin{array}{c}1 \% \mathrm{HF} \\
\# 6\end{array}$ & $\begin{array}{c}1 \% \mathrm{HF} \\
\# 7\end{array}$ \\
\hline \hline Acid Stage & 1 & 2 & 3 & 4 & 5 & 6 & 7 & 8 & 9 \\
\hline${ }^{9} \mathrm{Be}$ & $1.05 \mathrm{E}+02$ & $3.35 \mathrm{E}+01$ & $4.99 \mathrm{E}+02$ & $2.44 \mathrm{E}+02$ & $2.21 \mathrm{E}+02$ & $1.62 \mathrm{E}+02$ & $1.26 \mathrm{E}+02$ & $1.01 \mathrm{E}+02$ & $2.35 \mathrm{E}+01$ \\
$\mathrm{~B}$ & $2.10 \mathrm{E}+01$ & $1.12 \mathrm{E}+01$ & $1.36 \mathrm{E}+02$ & $4.95 \mathrm{E}+01$ & $3.80 \mathrm{E}+01$ & $3.02 \mathrm{E}+01$ & $2.07 \mathrm{E}-01$ & $2.34 \mathrm{E}+01$ & $7.86 \mathrm{E}-01$ \\
$\mathrm{Fe}$ & $2.36 \mathrm{E}+06$ & $5.72 \mathrm{E}+04$ & $2.23 \mathrm{E}+05$ & $8.26 \mathrm{E}+04$ & $7.96 \mathrm{E}+04$ & $5.98 \mathrm{E}+04$ & $4.35 \mathrm{E}+04$ & $1.71 \mathrm{E}+04$ & $5.44 \mathrm{E}+03$ \\
$\mathrm{Mn}$ & $3.60 \mathrm{E}+04$ & $1.78 \mathrm{E}+02$ & $6.27 \mathrm{E}+02$ & $2.61 \mathrm{E}+02$ & $2.42 \mathrm{E}+02$ & $1.93 \mathrm{E}+02$ & $1.44 \mathrm{E}+02$ & $5.35 \mathrm{E}+01$ & $1.40 \mathrm{E}+01$ \\
$\mathrm{Ca}$ & $3.14 \mathrm{E}+06$ & $2.26 \mathrm{E}+03$ & $4.58 \mathrm{E}+04$ & $1.32 \mathrm{E}+04$ & $1.15 \mathrm{E}+04$ & $1.30 \mathrm{E}+04$ & $9.05 \mathrm{E}+03$ & $1.64 \mathrm{E}+03$ & $1.83 \mathrm{E}+02$ \\
$\mathrm{Mg}$ & $9.89 \mathrm{E}+05$ & $2.47 \mathrm{E}+04$ & $6.84 \mathrm{E}+04$ & $1.21 \mathrm{E}+04$ & $1.48 \mathrm{E}+04$ & $3.00 \mathrm{E}+04$ & $3.03 \mathrm{E}+04$ & $1.03 \mathrm{E}+04$ & $3.19 \mathrm{E}+03$ \\
$\mathrm{Al}$ & $1.20 \mathrm{E}+06$ & $1.02 \mathrm{E}+05$ & $2.71 \mathrm{E}+06$ & $1.31 \mathrm{E}+06$ & $1.35 \mathrm{E}+06$ & $1.49 \mathrm{E}+06$ & $1.17 \mathrm{E}+06$ & $2.95 \mathrm{E}+05$ & $6.60 \mathrm{E}+04$ \\
$\mathrm{Ti}$ & $1.26 \mathrm{E}+03$ & $3.15 \mathrm{E}+03$ & $4.01 \mathrm{E}+04$ & $1.81 \mathrm{E}+04$ & $1.66 \mathrm{E}+04$ & $1.24 \mathrm{E}+04$ & $9.43 \mathrm{E}+03$ & $3.76 \mathrm{E}+03$ & $1.18 \mathrm{E}+03$ \\
$\mathrm{Na}$ & $2.72 \mathrm{E}+04$ & $6.19 \mathrm{E}+03$ & $2.72 \mathrm{E}+06$ & $1.07 \mathrm{E}+06$ & $8.68 \mathrm{E}+05$ & $7.97 \mathrm{E}+05$ & $5.91 \mathrm{E}+05$ & $1.05 \mathrm{E}+05$ & $1.69 \mathrm{E}+04$ \\
$\mathrm{~K}$ & $1.38 \mathrm{E}+05$ & $4.62 \mathrm{E}+04$ & $7.45 \mathrm{E}+05$ & $3.03 \mathrm{E}+05$ & $2.88 \mathrm{E}+05$ & $2.23 \mathrm{E}+05$ & $1.70 \mathrm{E}+05$ & $4.60 \mathrm{E}+04$ & $1.81 \mathrm{E}+04$ \\
\hline
\end{tabular}




\section{Table 4.4 1 gram subsample timeseries (ppm)}

This table contains the timeseries of each isotope tested in the 1-gram subsamples. Each sample was taken from the master sample HP-16-11 (RN35) after the master sample had been rinsed, dried, and massed following a chemical purification procedure. The data are presented in parts per million and graphed below in figures 4.7-4.10 and 4.12-4.17

\section{Gram}

Subsample

\begin{tabular}{|cccccccccccc|}
\hline $\begin{array}{c}\text { Sample } \\
\text { Name }\end{array}$ & RN46 & RN47 & RN48 & RN49 & RN50 & RN51 & RN52 & RN53 & RN54 & RN55 & RN35 \\
\hline \hline \multirow{2}{*}{ Acid Type } & Untreated & $\begin{array}{c}10 \% \mathrm{HCl} \\
\# 1\end{array}$ & $\begin{array}{c}10 \% \mathrm{HCl} \\
\# 2\end{array}$ & $\begin{array}{c}2.5 \% \mathrm{HF} \\
\# 1\end{array}$ & $\begin{array}{c}1 \% \mathrm{HF} \\
\# 2\end{array}$ & $\begin{array}{c}1 \% \mathrm{HF} \\
\# 3\end{array}$ & $\begin{array}{c}1 \% \mathrm{HF} \\
\# 4\end{array}$ & $\begin{array}{c}1 \% \mathrm{HF} \\
\# 5\end{array}$ & $\begin{array}{c}1 \% \mathrm{HF} \\
\# 6\end{array}$ & $\begin{array}{c}1 \% \mathrm{HF} \\
\# 7\end{array}$ & $\begin{array}{c}\text { Final } \\
\text { Sample }\end{array}$ \\
\hline \hline Acid Stage & 0 & 1 & 2 & 3 & 4 & 5 & 6 & 7 & 8 & 9 & 10 \\
\hline $9 \mathrm{Be}$ & $2.27 \mathrm{E}+01$ & $1.94 \mathrm{E}+01$ & $2.00 \mathrm{E}+01$ & $1.22 \mathrm{E}+01$ & $1.09 \mathrm{E}+01$ & $7.01 \mathrm{E}+00$ & $4.28 \mathrm{E}+00$ & $2.09 \mathrm{E}+00$ & $9.60 \mathrm{E}-01$ & $5.25 \mathrm{E}-01$ & $9.02 \mathrm{E}-01$ \\
$\mathrm{~B}$ & $2.92 \mathrm{E}+00$ & $2.44 \mathrm{E}+00$ & $2.65 \mathrm{E}+00$ & $1.83 \mathrm{E}+00$ & $1.81 \mathrm{E}+00$ & $1.38 \mathrm{E}+00$ & $1.23 \mathrm{E}+00$ & $1.29 \mathrm{E}+00$ & $1.08 \mathrm{E}+00$ & $8.37 \mathrm{E}-01$ & $5.57 \mathrm{E}-01$ \\
$\mathrm{Fe}$ & $5.90 \mathrm{E}+03$ & $1.32 \mathrm{E}+03$ & $1.47 \mathrm{E}+03$ & $9.32 \mathrm{E}+02$ & $7.54 \mathrm{E}+02$ & $5.04 \mathrm{E}+02$ & $3.68 \mathrm{E}+02$ & $2.22 \mathrm{E}+02$ & $1.62 \mathrm{E}+02$ & $1.28 \mathrm{E}+02$ & $1.50 \mathrm{E}+02$ \\
$\mathrm{Mn}$ & $6.91 \mathrm{E}+01$ & $4.08 \mathrm{E}+00$ & $4.51 \mathrm{E}+00$ & $3.03 \mathrm{E}+00$ & $2.72 \mathrm{E}+00$ & $1.73 \mathrm{E}+00$ & $1.12 \mathrm{E}+00$ & $6.73 \mathrm{E}-01$ & $6.07 \mathrm{E}-01$ & $4.67 \mathrm{E}-01$ & $1.16 \mathrm{E}+00$ \\
$\mathrm{Ca}$ & $5.05 \mathrm{E}+03$ & $2.76 \mathrm{E}+02$ & $3.20 \mathrm{E}+02$ & $1.45 \mathrm{E}+02$ & $1.74 \mathrm{E}+02$ & $1.10 \mathrm{E}+02$ & $4.63 \mathrm{E}+01$ & $1.54 \mathrm{E}+01$ & N/A & N/A & N/A \\
$\mathrm{Mg}$ & $2.83 \mathrm{E}+03$ & $8.84 \mathrm{E}+02$ & $1.05 \mathrm{E}+03$ & $6.39 \mathrm{E}+02$ & $5.12 \mathrm{E}+02$ & $3.46 \mathrm{E}+02$ & $2.36 \mathrm{E}+02$ & $1.38 \mathrm{E}+02$ & $8.14 \mathrm{E}+01$ & $6.40 \mathrm{E}+01$ & $7.23 \mathrm{E}+01$ \\
$\mathrm{Al}$ & $3.29 \mathrm{E}+04$ & $3.06 \mathrm{E}+04$ & $3.72 \mathrm{E}+04$ & $1.86 \mathrm{E}+04$ & $1.72 \mathrm{E}+04$ & $1.19 \mathrm{E}+04$ & $5.56 \mathrm{E}+03$ & $2.22 \mathrm{E}+03$ & $6.20 \mathrm{E}+02$ & $2.86 \mathrm{E}+02$ & $3.79 \mathrm{E}+02$ \\
$\mathrm{Ti}$ & $4.26 \mathrm{E}+02$ & $3.86 \mathrm{E}+02$ & $4.49 \mathrm{E}+02$ & $3.23 \mathrm{E}+02$ & $3.09 \mathrm{E}+02$ & $2.45 \mathrm{E}+02$ & $1.95 \mathrm{E}+02$ & $1.68 \mathrm{E}+02$ & $1.70 \mathrm{E}+02$ & $1.34 \mathrm{E}+02$ & $1.88 \mathrm{E}+02$ \\
$\mathrm{Na}$ & $1.53 \mathrm{E}+04$ & $1.62 \mathrm{E}+04$ & $1.99 \mathrm{E}+04$ & $9.25 \mathrm{E}+03$ & $8.73 \mathrm{E}+03$ & $6.41 \mathrm{E}+03$ & $2.42 \mathrm{E}+03$ & $7.72 \mathrm{E}+02$ & $9.39 \mathrm{E}+01$ & $5.40 \mathrm{E}+00$ & $2.87 \mathrm{E}+01$ \\
$\mathrm{~K}$ & $4.65 \mathrm{E}+03$ & $4.13 \mathrm{E}+03$ & $4.94 \mathrm{E}+03$ & $3.07 \mathrm{E}+03$ & $2.52 \mathrm{E}+03$ & $1.56 \mathrm{E}+03$ & $1.03 \mathrm{E}+03$ & $4.93 \mathrm{E}+02$ & $1.87 \mathrm{E}+02$ & $9.26 \mathrm{E}+01$ & $1.24 \mathrm{E}+02$ \\
\hline
\end{tabular}




\section{Table 4.5 Pre and post purification (}

This table contains the pre and post purification results for sample HP-16-11 (RN34 and RN35). The table also shows the data for purified sample RN35 normalized to 5 grams and 1 gram. These normalized data are plotted as the $10^{\text {th }}$ point in figures 4.7-4.10 and 4.12-4.17. Sample RN34 is a $5 \mathrm{~g}$ subsample of HP-16-11 and was immediately digested at the start of the experiment.

\begin{tabular}{|c|c|c|c|c|}
\hline Sample Name & RN34 & RN35 & RN35 & RN35 \\
\hline \hline Sample Point & $\begin{array}{c}5 \mathrm{~g} \text { Initial Sample } \\
(\mathrm{ppm})\end{array}$ & $\begin{array}{c}15 \mathrm{~g} \text { Final } \\
\text { Sample }(\mathrm{ppm})\end{array}$ & $\begin{array}{c}\text { Normalized to } \\
5 \mathrm{~g}(\mathrm{ppm})\end{array}$ & $\begin{array}{c}\text { Normalized to } \\
1 \mathrm{~g}(\mathrm{ppm})\end{array}$ \\
\hline $\mathrm{Be}$ & $7.23 \mathrm{E}+00$ & $1.74 \mathrm{E}+00$ & $5.79 \mathrm{E}-01$ & $1.16 \mathrm{E}-01$ \\
$\mathrm{~B}$ & $7.60 \mathrm{E}+00$ & $3.08 \mathrm{E}+01$ & $1.03 \mathrm{E}+01$ & $2.06 \mathrm{E}+00$ \\
$\mathrm{Fe}$ & $1.81 \mathrm{E}+04$ & $2.25 \mathrm{E}+03$ & $7.49 \mathrm{E}+02$ & $1.50 \mathrm{E}+02$ \\
$\mathrm{Mn}$ & $2.20 \mathrm{E}+02$ & $1.74 \mathrm{E}+01$ & $5.81 \mathrm{E}+00$ & $1.16 \mathrm{E}+00$ \\
$\mathrm{Ca}$ & $1.54 \mathrm{E}+04$ & $6.02 \mathrm{E}+02$ & $2.01 \mathrm{E}+02$ & $4.02 \mathrm{E}+01$ \\
$\mathrm{Mg}$ & $4.61 \mathrm{E}+03$ & $1.08 \mathrm{E}+03$ & $3.61 \mathrm{E}+02$ & $7.23 \mathrm{E}+01$ \\
$\mathrm{Al}$ & $6.64 \mathrm{E}+04$ & $5.69 \mathrm{E}+03$ & $1.90 \mathrm{E}+03$ & $3.79 \mathrm{E}+02$ \\
$\mathrm{Ti}$ & $1.15 \mathrm{E}+03$ & $2.81 \mathrm{E}+03$ & $9.38 \mathrm{E}+02$ & $1.88 \mathrm{E}+02$ \\
$\mathrm{Na}$ & $4.34 \mathrm{E}+04$ & $4.31 \mathrm{E}+02$ & $1.44 \mathrm{E}+02$ & $2.87 \mathrm{E}+01$ \\
$\mathrm{~K}$ & $1.46 \mathrm{E}+04$ & $1.86 \mathrm{E}+03$ & $6.20 \mathrm{E}+02$ & $1.24 \mathrm{E}+02$ \\
\hline
\end{tabular}

\subsubsection{Chemical series $1(10 \% \mathrm{HCl} \mathrm{X} \mathrm{2)}$}

Opaque mineral veneers and exposed inclusions are visibly removed in chemical series 1 . The overall quartz grain size is not dramatically reduced, or fundamentally altered (Figure 4.6). This sequence represents $\sim 14 \%$ of the total mass loss. The target of this acid stage is the removal of opaque/oxide, phyllosilicate, and carbonate minerals. These minerals contain an abundance of $\mathrm{Fe}, \mathrm{Mg}, \mathrm{Ca}$, and $\mathrm{Mn}$, small amounts of $\mathrm{Al}$ and $\mathrm{K}$, and trace amounts of other rare earth elements. The total concentration timeseries for $\mathrm{Fe}, \mathrm{Ca}, \mathrm{Mn}$, and $\mathrm{Mg}$ are shown in figures 4.7-4.10. The removal of opaque mineral veneers and inclusions is documented in figure 4.6. It shows the successful leaching of material from an untreated sample (Figure 4.6A) to an $\mathrm{HCl}$ treated sample (Figure 4.6B). 

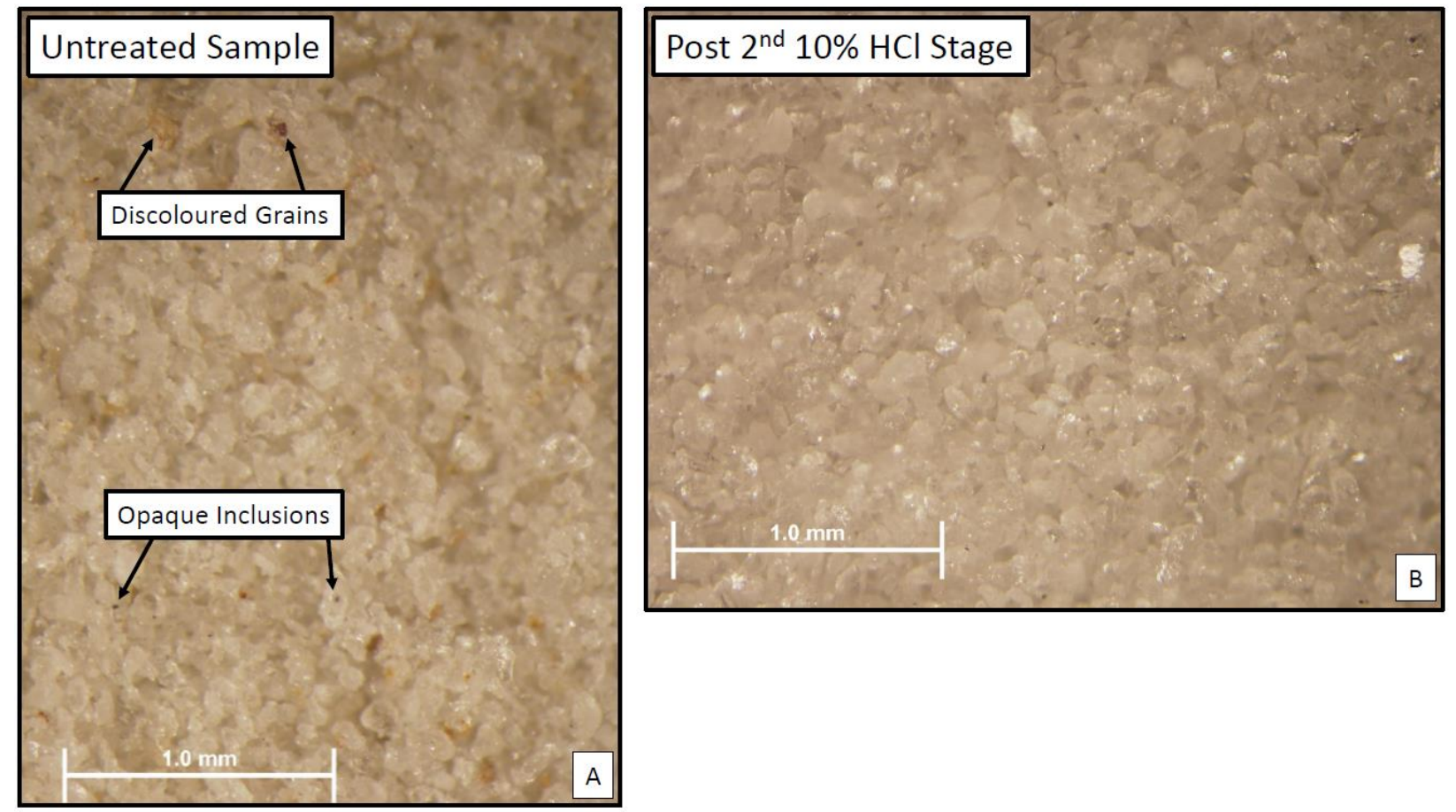

\section{Figure 4.6 Post HCl photomicrograph}

Minerals mantling the quartz grains are removed by the first $\mathrm{HCl}$ bath. Photo A shows the untreated sample which has abundant mineral overgrowths and inclusions exposed at the surface of the mineral grains. Photo B shows the same sample after two subsequent 24 hour 10\% $\mathrm{HCl}$ leaches. By the end of the $\mathrm{HCl}$ phase all opaque minerals have been removed as well as any inclusions exposed at the surface of the grain. There is no obvious grain size reduction of the quartz, but this stage is still responsible for $\sim 5 \%$ of the total mass reduction required for the quartz purification process.

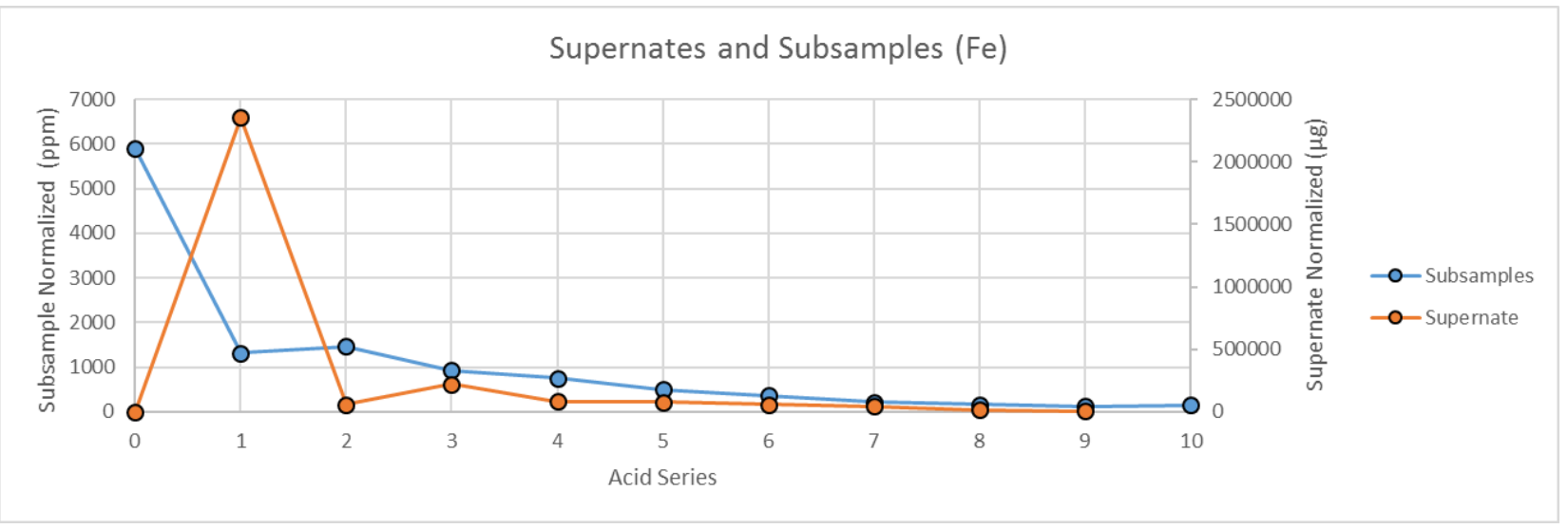

\section{Figure 4.7 Fe timeseries}

This graph is a timeseries of both the $1 \mathrm{~g}$ subsample and supernates. The majority of Fe is removed during acid stage 1 , the first $\mathrm{HCl}$ leach. The large amount of Fe removed is likely due to a combination of oxide removal and phyllosilicate digestion. A smaller amount of Fe is removed during the HF leaches (acid stages 3-9); this is likely the result of oxide inclusions being removed as the HF encounters them. 


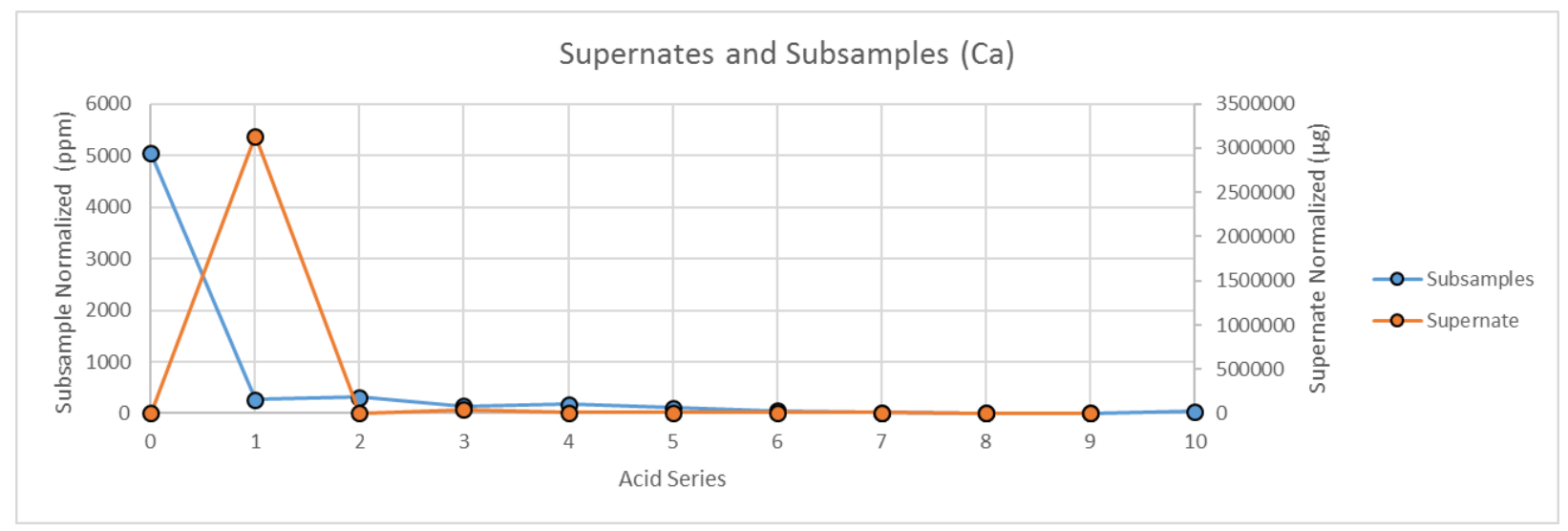

\section{Figure 4.8 Ca timeseries}

This graph is a timeseries of both the $1 \mathrm{~g}$ subsample and supernates. The majority of $\mathrm{Ca}$ is removed during acid stage 1 , the first $\mathrm{HCl}$ leach. The large amount of $\mathrm{Ca}$ removed is likely due phyllosilicate digestion.

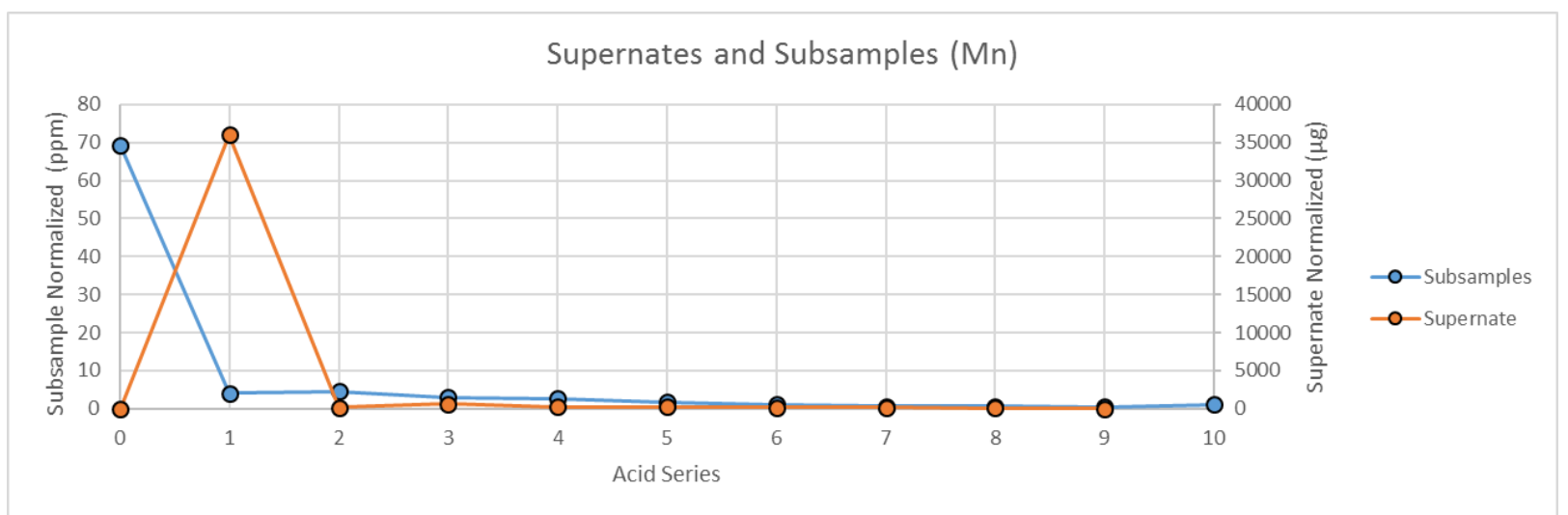

\section{Figure 4.9 Mn timeseries}

This graph is a timeseries of both the $1 \mathrm{~g}$ subsample and supernates. The majority of $\mathrm{Mn}$ is removed during acid stage 1 , the first $\mathrm{HCl}$ leach. The large amount of Mn removed is likely due to phyllosilicate digestion.

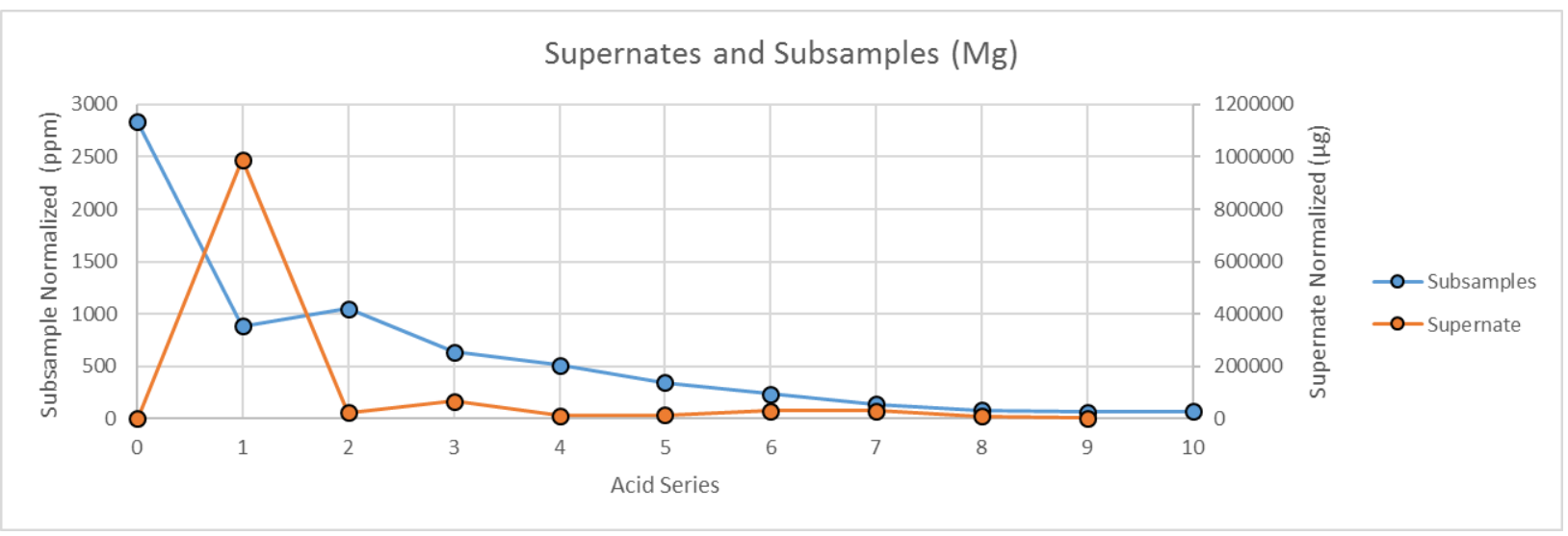

Figure 4.10 Mg timeseries

This graph is a timeseries of both the $1 \mathrm{~g}$ subsample and supernates. The majority of $\mathrm{Mg}$ is removed during acid stage 1 , the first $\mathrm{HCl}$ leach. The large amount of $\mathrm{Mg}$ removed is likely due to a combination of oxide removal and phyllosilicate digestion. A smaller amount of Mg is removed during the HF leaches (acid stages 3-9) this is likely the result of oxide inclusions being removed as the HF encounters them 


\subsubsection{Chemical Series 2 and $3(2.5 \% \mathrm{HF}$ and $1 \% \mathrm{HF}$ X 6$)$}

Chemical phases two and three are the main grain reduction intervals where any quartz overgrowths containing meteoric ${ }^{10} \mathrm{Be}$ are removed from the sample (Figure 4.11). This process visibly reduces the overall grain size and enlarges any small fractures or slight mineralogical discontinuities to which meteoric ${ }^{10} \mathrm{Be}$ may be adsorbed (Figure 4.11). This phase of chemical treatment is responsible for $\sim 86 \%$ of the mass loss (Figure 4.5 ). The HF phases are critical to reducing the overall abundance of $\mathrm{Al}, \mathrm{Ti}, \mathrm{Na}$, and $\mathrm{K}$ which hamper column chemistry and can inhibit successful elution of Be during column chemistry (Ochs and Ivy-Ochs, 1997; Corbett et al. 2016) and removing meteoric ${ }^{10} \mathrm{Be}$ which is indistinguishable in an accelerator mass spectrometer from in-situ ${ }^{10} \mathrm{Be}$. The total concentration timeseries for $\mathrm{Al}, \mathrm{Ti}, \mathrm{Na}$, and $\mathrm{K}$ are shown in figures 4.12-4.15. Additionally, several careful applications of low concentration HF is essential to not destroying the sample (Brown et al, 1991). A sample can be rendered unusable if the total quartz mass is too low to retain enough in-situ ${ }^{10} \mathrm{Be}$ to count on an accelerator mass spectrometer or if all of the quartz has been completely dissolved by HF. 


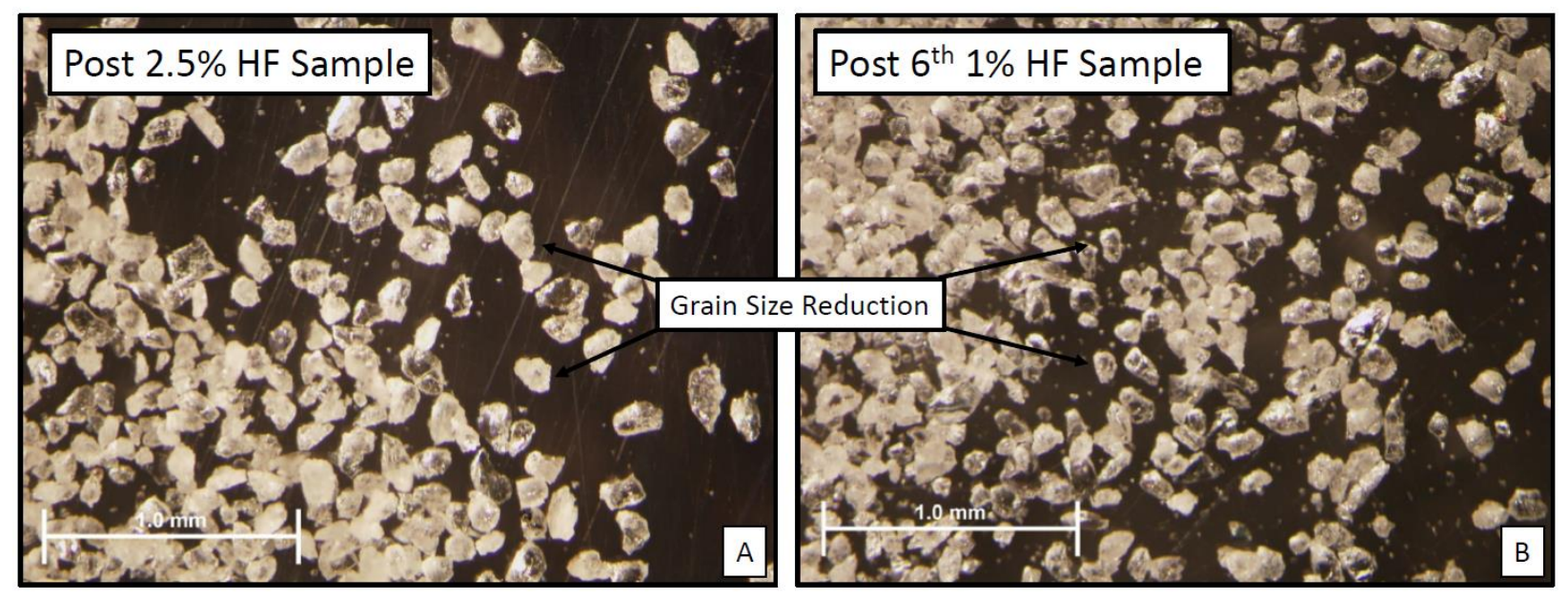

\section{Figure 4.11 Post HF photomicrograph}

The sample undergoes significant grain size reduction as it progresses through the $1 \% \mathrm{HF}$ leaching cycles. This transition is apparent between image A and image B. Image A was taken after the initial 2.5\% HF leach and image B shows the same sample after the $6^{\text {th }}$ and final $1 \% \mathrm{HF}$ leach. The total mass loss represented here is $20 \%$ of the overall mass reduction required by the quartz purification process. The $2.5 \% \mathrm{HF}$ phase alone is responsible for $10 \%$ of the total grain size reduction required for pure quartz.

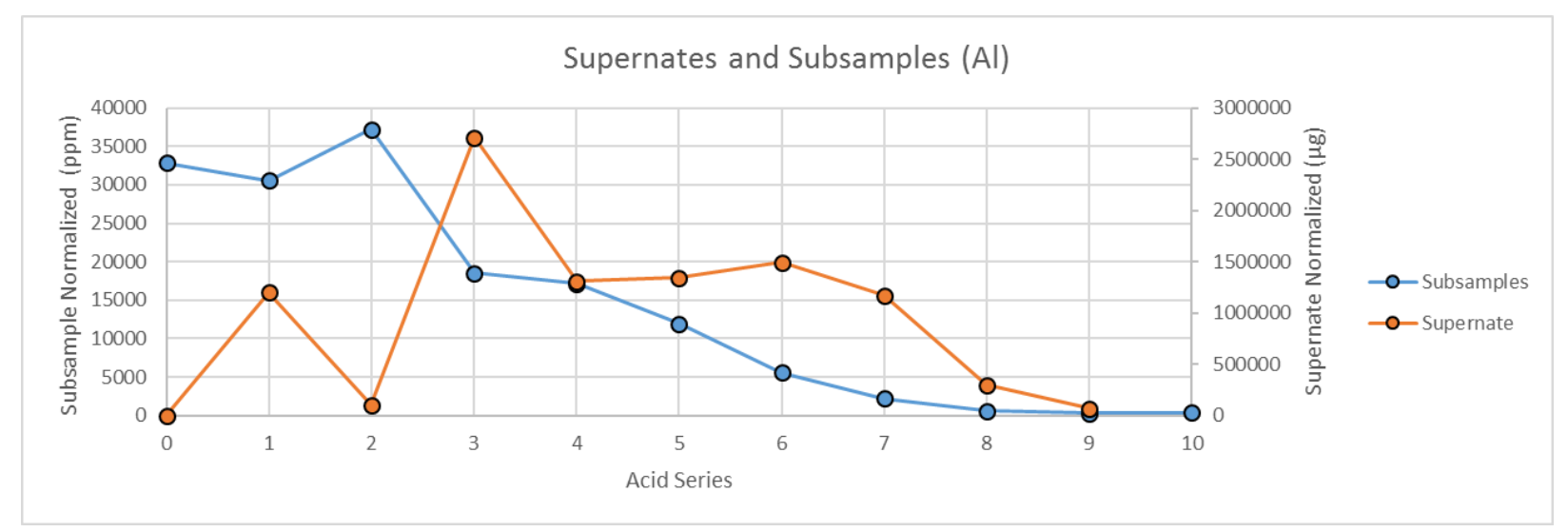

Figure 4.12 Al timeseries

This graph is a timeseries of both the $1 \mathrm{~g}$ subsample and supernates. The majority of $\mathrm{Al}$ is removed from the $1 \mathrm{~g}$ subsamples during the HF phases (3-9) while the Al signal in the supernate has two peaks and remains high through most of the HF leaching process. 


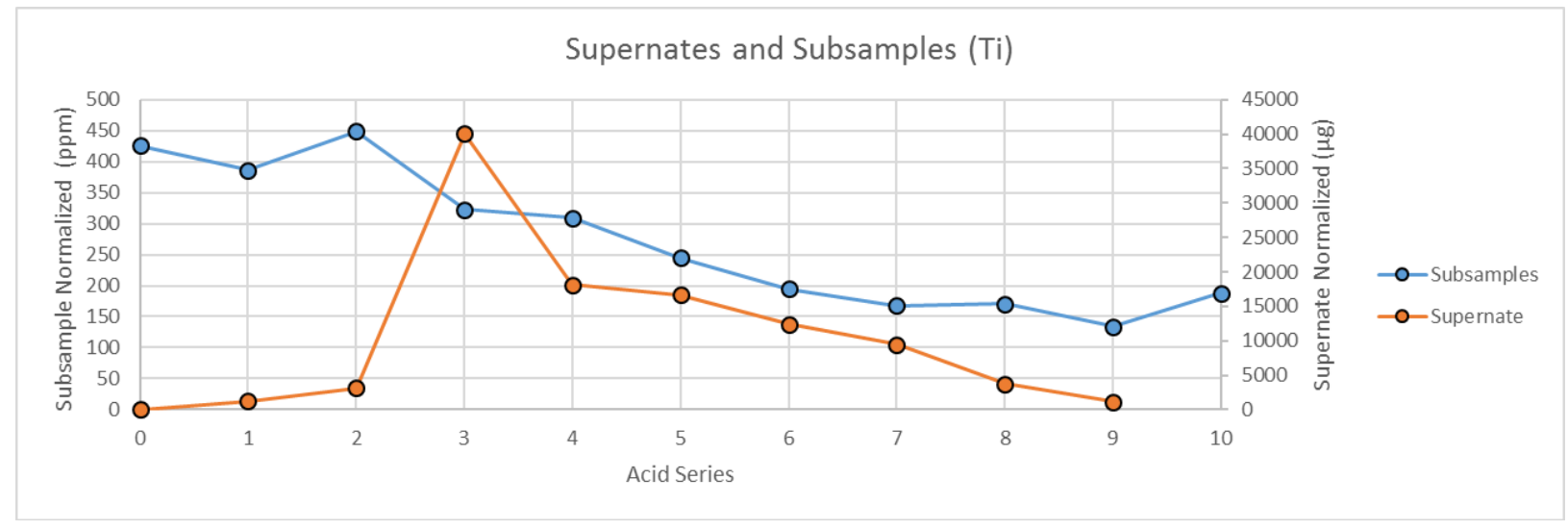

Figure 4.13 Ti timeseries

This graph is a timeseries of both the $1 \mathrm{~g}$ subsample and supernates. The majority of $\mathrm{Ti}$ is removed from the $1 \mathrm{~g}$ subsamples during the HF phases (3-9). The supernate data show that Ti is removed dominantly during the HF phases (3-9).

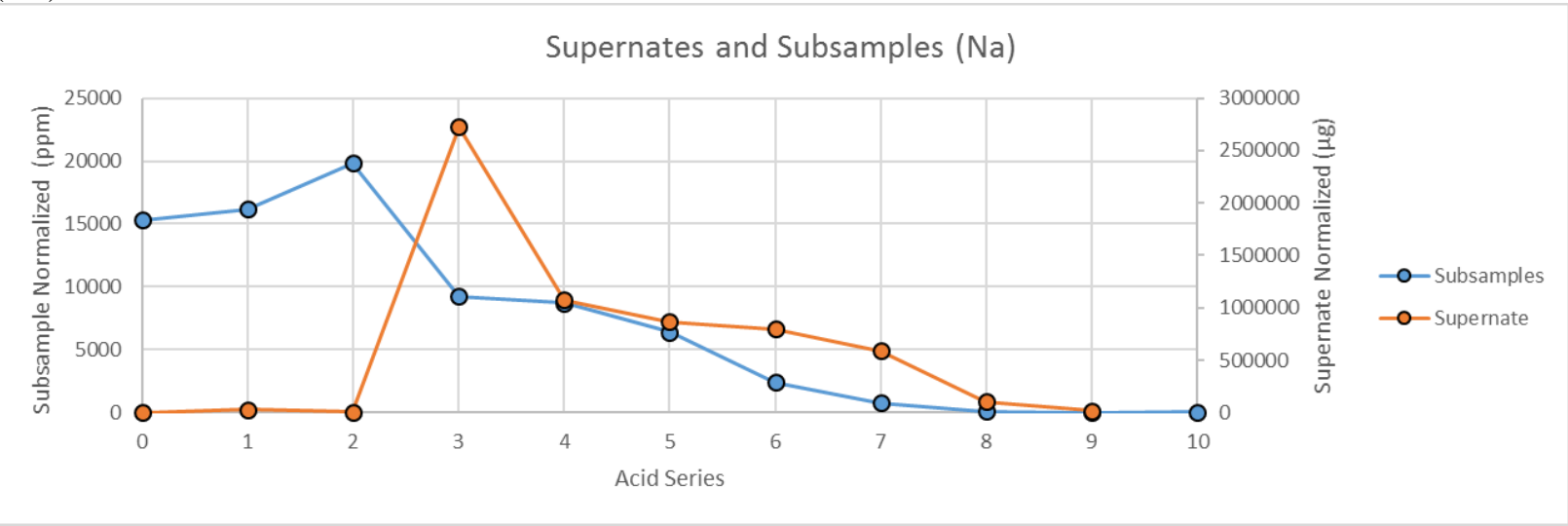

\section{Figure 4.14 Na timeseries}

This graph is a timeseries of both the $1 \mathrm{~g}$ subsample and supernates. $\mathrm{Na}$ is removed from the $1 \mathrm{~g}$ subsamples during the HF phases (3-9). The supernate data show that Na is removed dominantly during the HF phases (3-9).

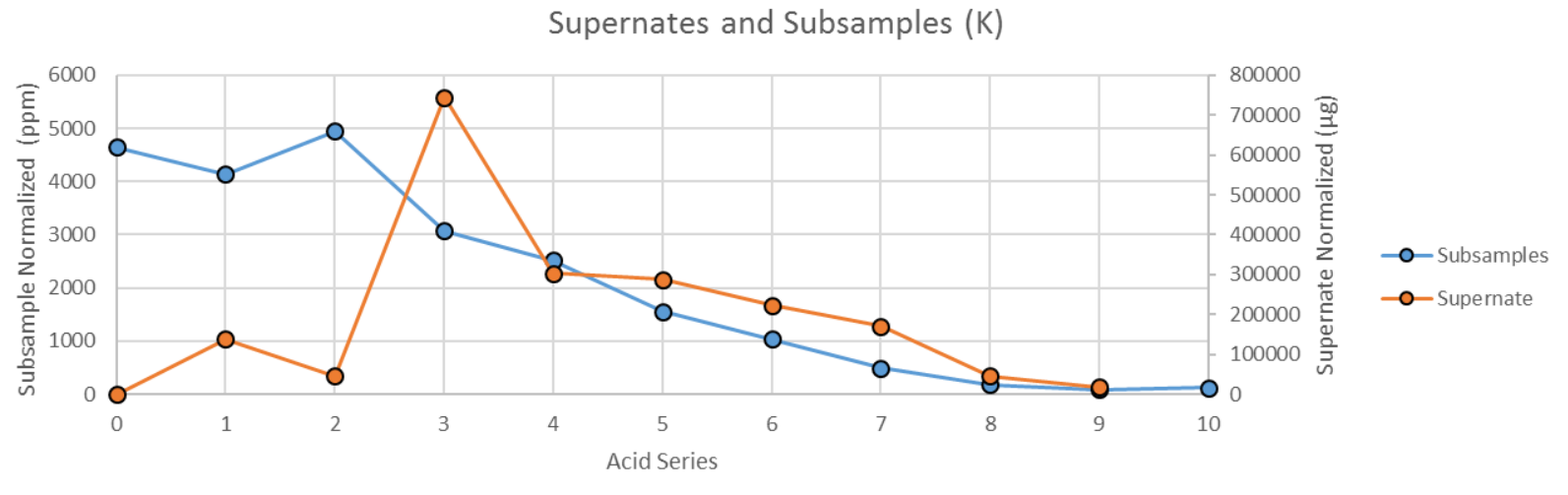

Figure 4.15 K timeseries

This graph is a timeseries of both the $1 \mathrm{~g}$ subsample and supernates. The majority of $\mathrm{K}$ is removed from the $1 \mathrm{~g}$ subsamples during the HF phases (3-9). The supernate data show that $\mathrm{K}$ is removed dominantly during the HF phases (3-9). 


\subsubsection{Beryllium and Boron}

${ }^{9} \mathrm{Be}$ is leached throughout the purification process. The ${ }^{9} \mathrm{Be}$ and ${ }^{10} \mathrm{~B}$ timeseries show constant ion-load reduction (Figures 4.12 and 4.17). Overall, this is unsurprising given that both elements are soluble in acidic solutions. However, it is remarkable how little ${ }^{10} \mathrm{~B}$ is removed generally throughout chemistry. In comparison, the ${ }^{9} \mathrm{Be}$ is consistently leached through time with each acid stage.

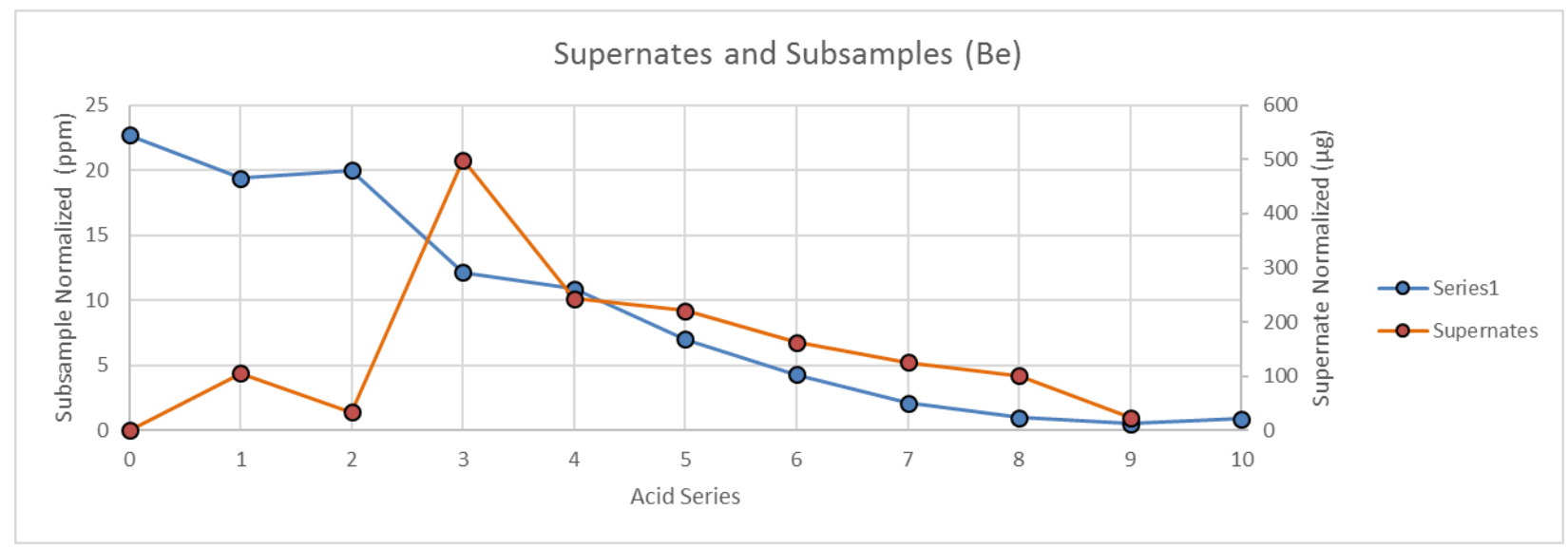

Figure 4.16 ${ }^{9}$ Be timeseries

This graph is a timeseries of both the $1 \mathrm{~g}$ subsample and supernates. The $1 \mathrm{~g}$ subsamples show that ${ }^{9} \mathrm{Be}$ is removed by each acid stage.

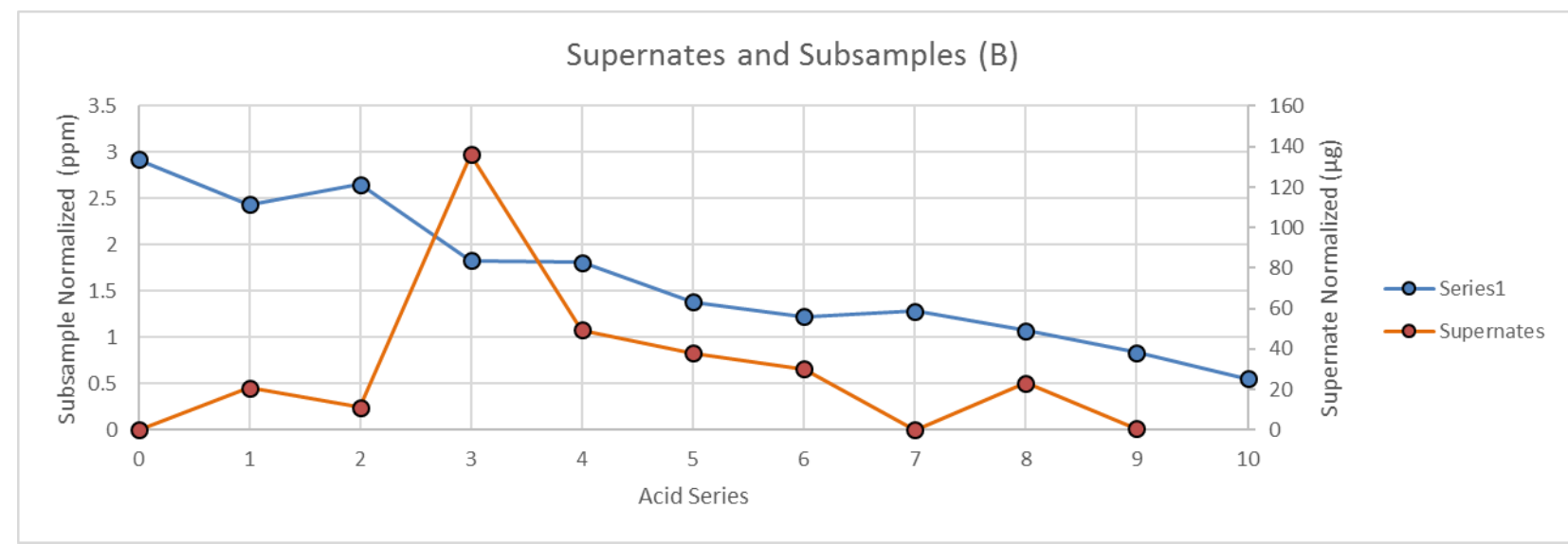

\section{Figure 4.17 B timeseries}

This graph is a timeseries of both the $1 \mathrm{~g}$ subsample and supernates. The $1 \mathrm{~g}$ subsamples show that $\mathrm{B}$ is removed by each acid stage.

Comparison of the quantity of ${ }^{10} \mathrm{~B}$ and ${ }^{9} \mathrm{Be}$ in the initial $5 \mathrm{~g}$ of untreated material and the final $14 \mathrm{~g}$ of treated material is a good gauge as to the effectiveness of the method. Comparison of 
the normalized data shows a $96 \%$ reduction of ${ }^{9} \mathrm{Be}$ over the course of the cleaning (Figure 4.18).

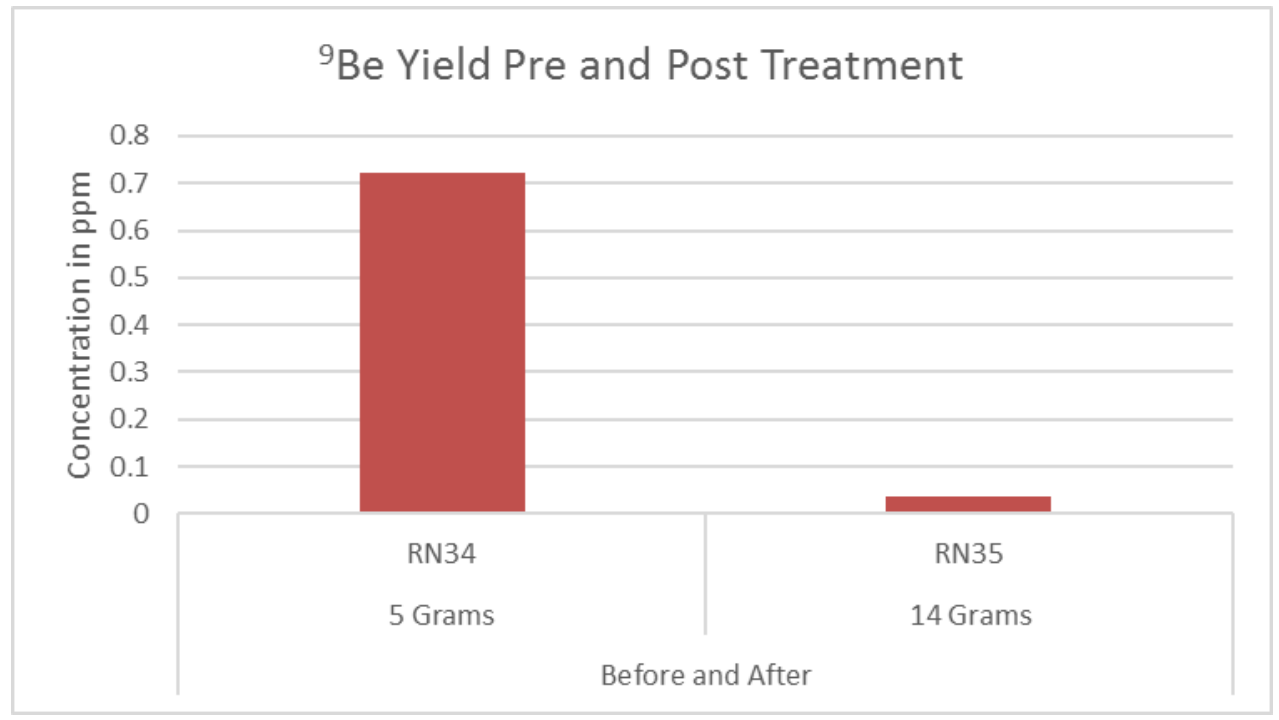

\section{Figure 4.18 Graph of normalised Be data}

The total reduction of ${ }^{9} \mathrm{Be}$ from the sample is $96 \%$ using this quartz purification process. The bar on the right is the initial untreated $5 \mathrm{~g}$ subsample and the right bar is the final $15 \mathrm{~g}$ sub sample normalized to $5 \mathrm{~g}$.

\subsubsection{Major Elements Removed by $\mathrm{HCl}(\mathrm{Fe}, \mathrm{Ca}, \mathrm{Mg}$, and $\mathrm{Mn})$}

The $\mathrm{HCl}$ series removes six of the major elements measured for this study. Most the work is done in the initial $\mathrm{HCl}$ leach (acid stage 1); the second $\mathrm{HCl}$ leach (acid stage 2) is a precaution to ensure that all grain coatings, phyllosilicates, and exposed inclusions have been dissolved. This phase focuses on the removal of $\mathrm{Fe}, \mathrm{Mn}, \mathrm{Ca}$, and $\mathrm{Mg}$. These elements form several oxide and carbonate minerals which react with dilute $\mathrm{HCl}$. A distinct pulse in $\mathrm{Al}$ and $\mathrm{K}$ likely represents the removal of small phyllosilicates during acid stage 1. By comparing the initial untreated sample with the treated sample, we see that $98 \%$ of $\mathrm{Fe}, 99 \%$ of $\mathrm{Mg}, 99 \%$ of $\mathrm{Ca}$, and $95 \%$ of Mn are removed. 


\subsubsection{Major Elements removed by $\mathrm{HF}(\mathrm{Al}, \mathrm{Ti}, \mathrm{Na}$, and $\mathrm{K})$}

The HF series of cleaning is responsible for removing four primary elements from the samples. $\mathrm{HF}$ reduces the amount of $\mathrm{Al}, \mathrm{Ti}, \mathrm{Na}$, and $\mathrm{K}$ from the samples by active grain reduction throughout the leaching process. During the initial $\mathrm{HCl}$ timeseries some $\mathrm{Al}$ and $\mathrm{K}$ is removed as phyllosilicates are dissolved, however the most significant loss occurs with the addition of HF. The first three 1\% HF leaches (acid stages 4-6) saturate with Al (Figure 4.12) then subsequently reduce with the next three $1 \%$ HF leaches (acid stages 7-9). As the timeseries progresses the removal of $\mathrm{Fe}$ and $\mathrm{Mg}$ continues into the HF stages, potentially representing the progressive exposure and dissolution of small opaque mineral inclusions present in the quartz grains. By comparing the initial untreated sample with the treated sample, we see that $98 \%$ of $\mathrm{Al}, 52 \%$ of $\mathrm{Ti}, 100 \%$ of $\mathrm{Na}$, and $97 \%$ of $\mathrm{K}$ are removed.

\subsubsection{Accelerator mass spectrometer data}

This section explores only the removal of ${ }^{10} \mathrm{Be}$ throughout the quartz purification timeseries. This time series is important because it demonstrates that all meteoric ${ }^{10} \mathrm{Be}$ is removed by the end of the procedure at $\sim 35 \%$ mass loss, and therefore, illustrates the reliability of the newly developed method.

\subsubsection{Meteoric ${ }^{10} \mathrm{Be}$}

The majority of ${ }^{10} \mathrm{Be}$ is removed during the initial $\mathrm{HCl}$ leaching stages (Figure 4.20 and 4.21). This large amount of ${ }^{10} \mathrm{Be}$ is likely produced in the atmosphere, because meteoric ${ }^{10} \mathrm{Be}$ is 1000 times more abundant in nature (Monaghan et al., 1985/86; Brown et al., 1991) than in-situ ${ }^{10} \mathrm{Be}$. Meteoric ${ }^{10} \mathrm{Be}$ can either adsorb to the outside of a quartz grain (Gosse and Phillips, 2001; Dunai, 2010) or be incorporated during the formation of oxide minerals which can coat a quartz grain (Freemann and Weed, 1987; Brown et al., 1991). These data show that the majority of the meteoric ${ }^{10} \mathrm{Be}$ is removed in the first three acid stages and agrees well with Kohl and 
Nishiizumi (1992). However, it is critical that the majority of $\mathrm{Ti}, \mathrm{Al}, \mathrm{Na}$, and $\mathrm{K}$ are leached from the sample in subsequent acid stages, the sample may not be appropriate for digestion after the first three acid series and may fail in at other stages during chemical processing. 
Table 4.6 $\quad{ }^{10} \mathrm{Be}$ timeseries pre and post purification $(\mathrm{ppm})$

This table contains the timeseries of ${ }^{10} \mathrm{Be}$ removed during the purification procedure. The accelerator mass spectrometer results are shown.

The Sample RN35 is shown as the cleaned sample in the below table. Some acid supernates were combined.

\begin{tabular}{|c|c|c|c|c|c|c|c|c|c|}
\hline $\begin{array}{l}\text { Sample } \\
\text { Name }\end{array}$ & Acid Stage & $\begin{array}{c}{ }^{10} \mathrm{Be} /{ }^{9} \mathrm{Be} \\
\left(10^{-15}\right)\end{array}$ & $\begin{array}{l}\text { Uncertainty } \\
\quad\left(\mathbf{1 0}^{-15}\right)\end{array}$ & $\begin{array}{c}\% \\
\text { Uncertainty }\end{array}$ & $\begin{array}{c}\text { Max }{ }^{9} \text { Be } \\
\text { Current } \\
\text { (nanoAmps) }\end{array}$ & $\begin{array}{c}\text { Min }{ }^{9} \mathbf{B e} \\
\text { Current } \\
\text { (nanoAmps) }\end{array}$ & $\begin{array}{c}\text { Avg. }{ }^{9} \text { Be } \\
\text { Current } \\
\text { (nanoAmps) }\end{array}$ & $\begin{array}{c}\text { Fraction } \\
\text { of } \\
\text { Standard } \\
\text { Current }\end{array}$ & $\begin{array}{c}\text { Total } \\
{ }^{10} \mathrm{Be} \\
\text { counts }\end{array}$ \\
\hline RN36-37 & Stage1\&2 & $6.08 \mathrm{E}+08$ & 10259192 & 1.7 & 728 & 352 & 578 & 0.064 & 32849 \\
\hline RN38 & Stage 3 & 319242.8 & 17713.2 & 5.5 & 3233 & 1977 & 2719 & 0.300 & 358 \\
\hline RN39-40 & Stage $4 \& 5$ & 169251.6 & 18085.14 & 10.7 & 3663 & 2033 & 2932 & 0.324 & 140 \\
\hline RN41-RN42 & Stage6\&7 & 140233 & 15214.32 & 10.8 & 4525 & 1029 & 3086 & 0.341 & 105 \\
\hline RN43-RN44 & Stage8\&9 & 140024.4 & 10437.64 & 7.5 & 8737 & 6847 & 7684 & 0.848 & 269 \\
\hline RN35 & Cleaned Sample & 120435.2 & 3260.347 & 2.7 & 9562 & 7995 & 9155 & 1.011 & 1957 \\
\hline RN45 & Blank & 0.00 & 0.00 & 0.0 & 10300 & 7867 & 9613 & 1.061 & 34 \\
\hline
\end{tabular}




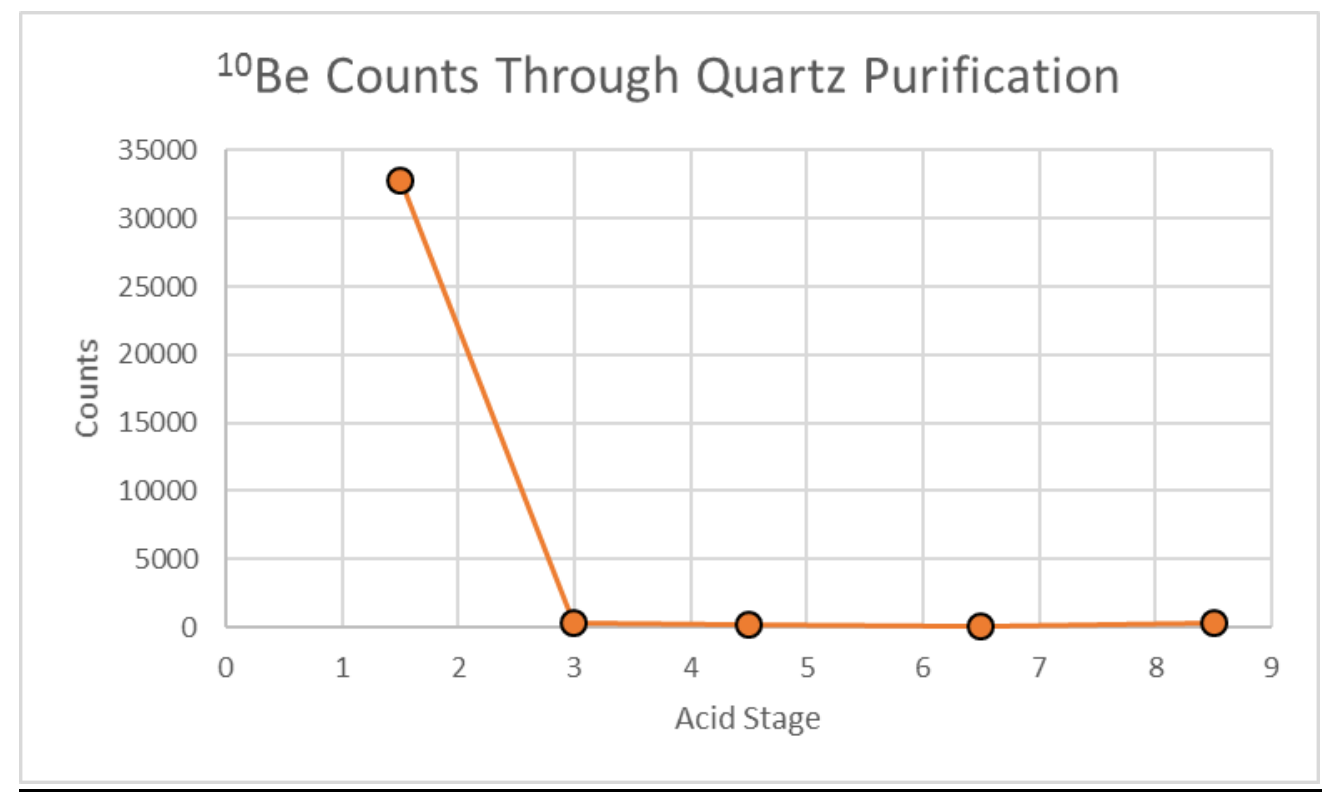

Figure 4.19 ${ }^{10} \mathrm{Be}$ purification timeseries

This graph is a timeseries of the $1 \mathrm{~g}$ subsample of ${ }^{10} \mathrm{Be}$ removal during purification. The majority of ${ }^{10} \mathrm{Be}$ is removed during the $\mathrm{HCl}$ stages (1 and 2). Samples are plotted based combined acid stages (e.g. Acid Stages 1 and 2 were combined and are plotted as Acid Stage 1.5).

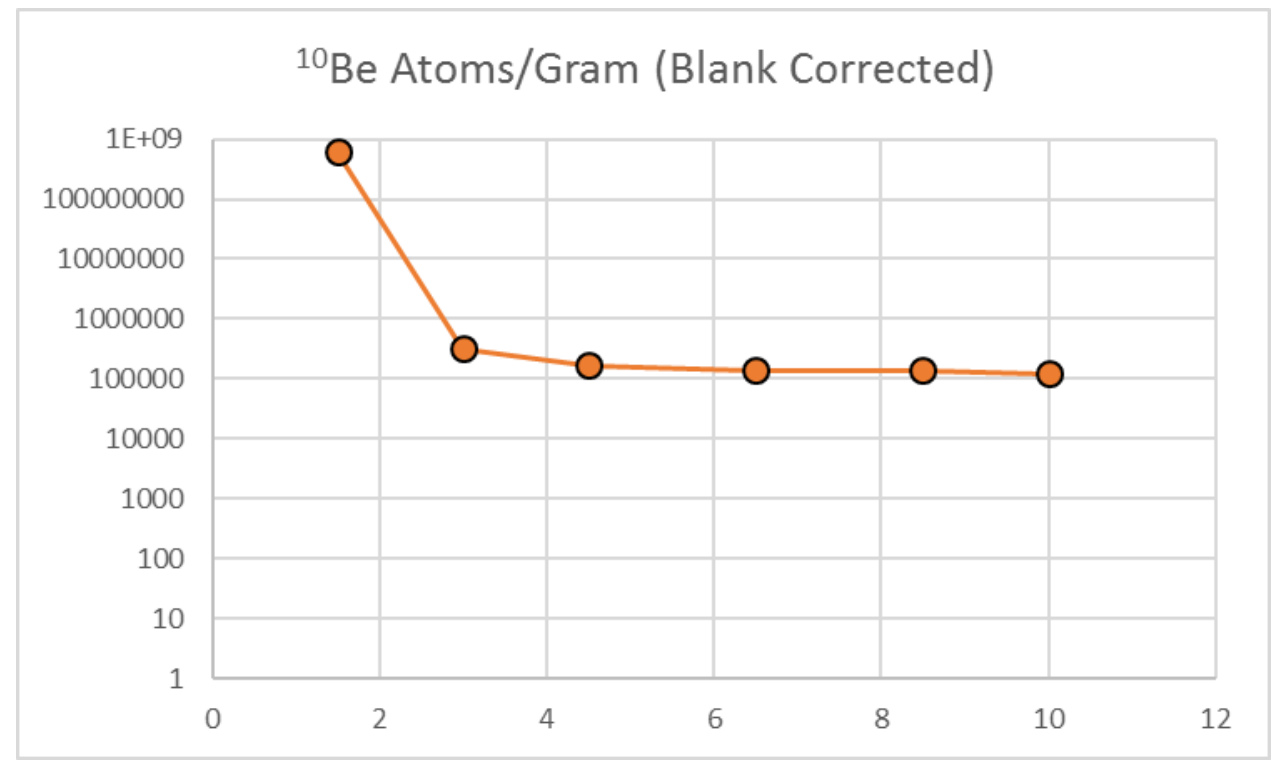

Figure 4.20 Blank corrected ${ }^{10} \mathrm{Be}$ atoms/gram semi-log plot

This graph shows a timeseries of the quartz purification for meteoric 10Be. The total counts from acid stage 3 to acid stage 7 are likely suppressed by weak beam current strength. 


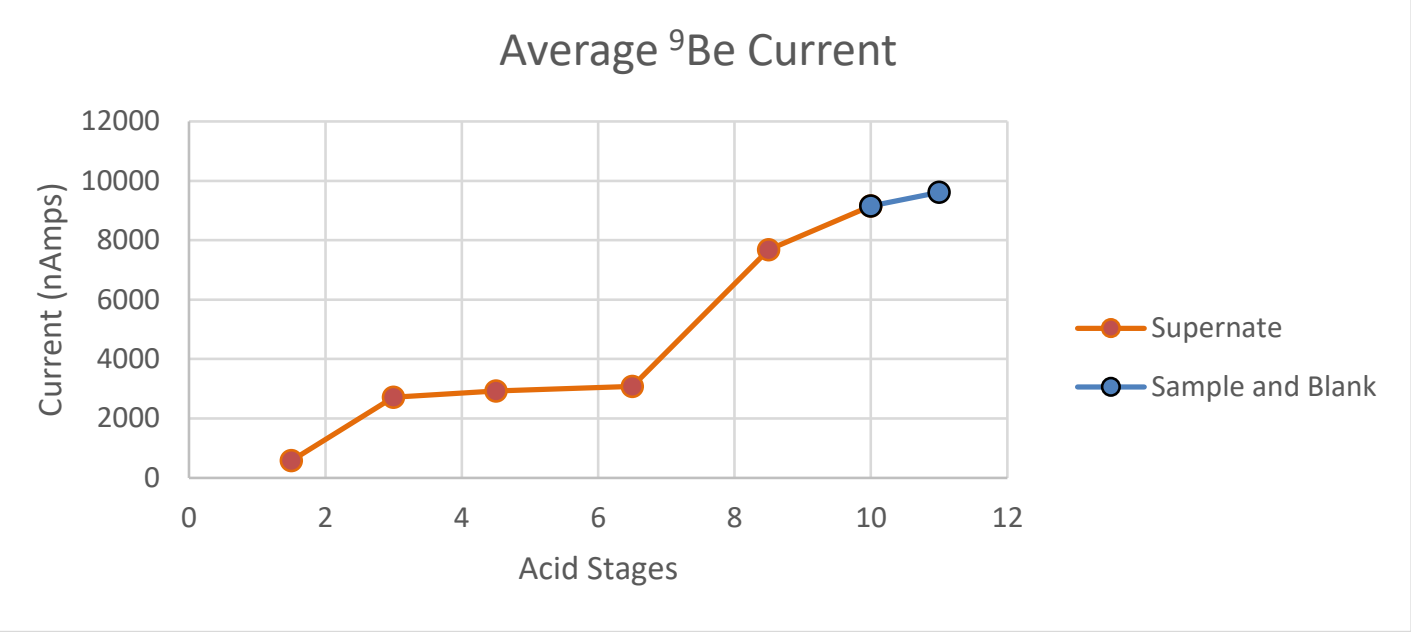

\section{Figure 4.21 Current strength timeseries}

This graph is a timeseries of the accelerator mass spectrometry beam current while the supernate samples were run. As the samples are cleaned the beam strength increases.

The ${ }^{9} \mathrm{Be}$ beam strength increases as the samples become purer (Figure 4.21) and leads to an increase in the total measured counts (Figure 4.20). Acid stage 8 shows a significant increase in beam strength while simultaneously showing a drop in Al concentration (Figure 4.22). 


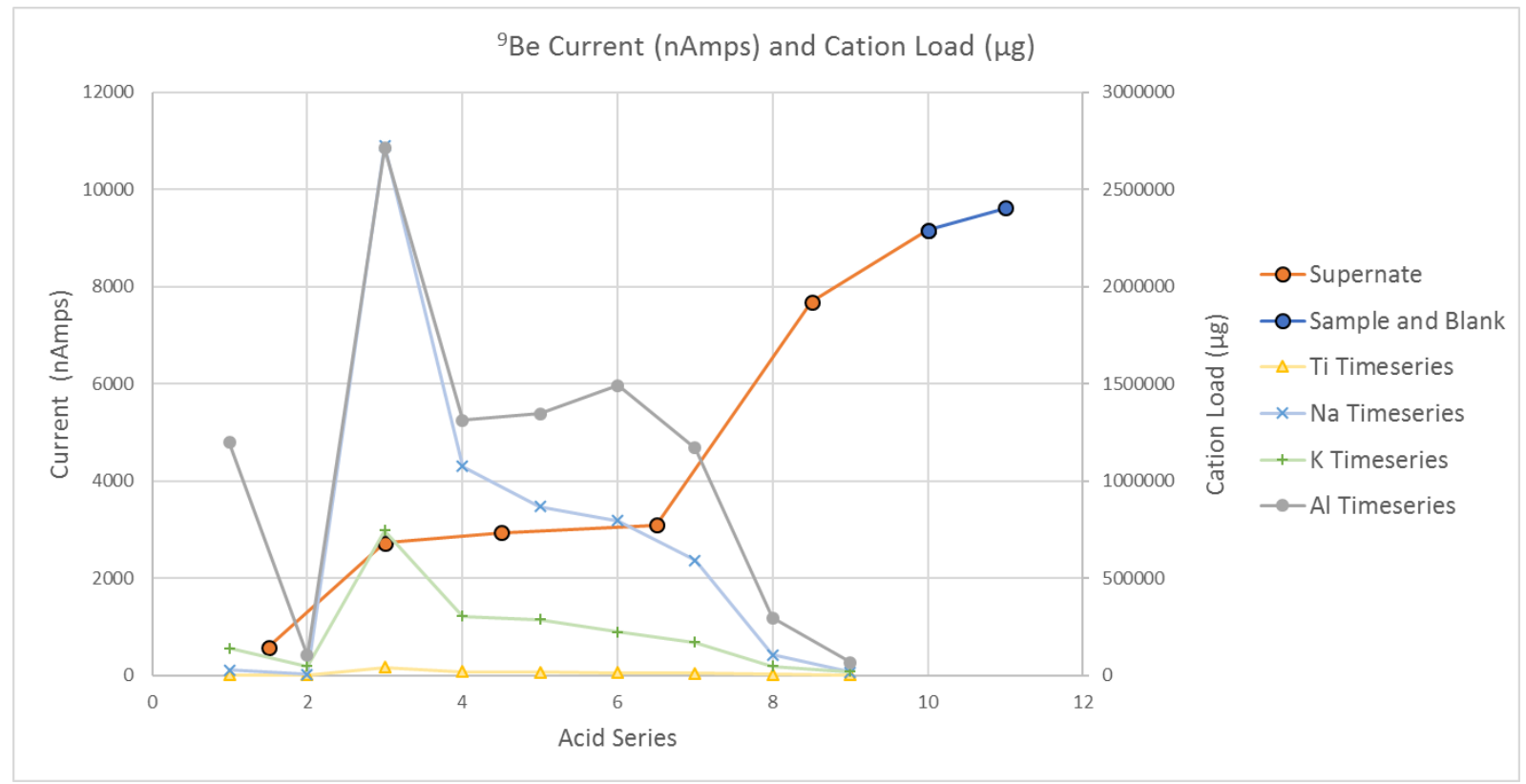

Figure 4.22 Beam current and cation load

The orange line and points are supernates, the blue line and points are purified sample and blank, the grey line and points are $\mathrm{Al}$ in the supernates, the green line and points are $\mathrm{K}$ in the supernates, the lite blue line and points are $\mathrm{Na}$ in the supernates, the yellow line and points are $\mathrm{Ti}$ in the supernates.

\subsubsection{Digestion and Column Chemistry}

The treated fine-grained quartz sample produced a similar amount of post-digestion residual material as the coarser grained equivalent samples. Surface area increase for this sample was not an issue during digestion, however, subsequent fine- grained samples have had extreme exothermic reactions with the addition of $50 \% \mathrm{HF}$. The residual material left by this treatment procedure is minimal when compared to the 5-gram untreated subsample (Figure 4.23). The amount of material removed by this cleaning protocol is documented by sample numbers RN34 and RN35, found in table 4.5 and shown in figure 4.23. Accelerator mass spectrometry beam current results show that HP-16-11 (RN35) preformed as well or better than other coarsegrained material processed via standard methods (Table 4.7 and Figure 4.24). 


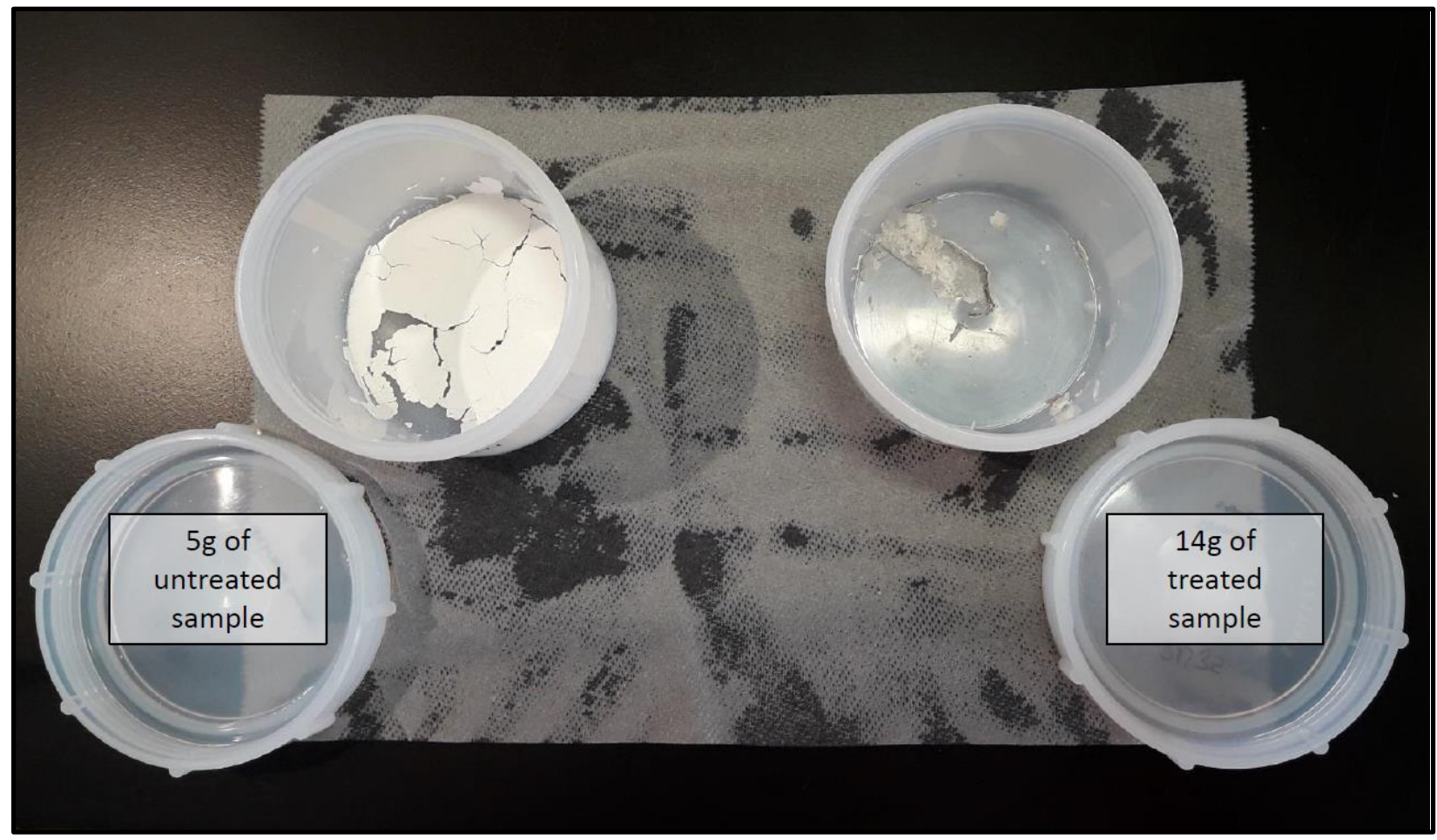

Figure 4.23 Pre- and post-treatment

Fourteen grams of treated sample produce a significantly smaller post digestion residue then 5 grams of untreated material. The $14 \mathrm{~g}$ sample shows that fine-grained monomineralic quartz can be sufficiently treated to produce a clean and usable sample for ${ }^{10} \mathrm{Be}$ dating.

\section{Table 4.7 Sample comparison}

The table contains the beam strength (nAmps), the total ${ }^{10} \mathrm{Be}$ counts, and the total grams digested. Sample RN35 (yellow) is compared with granite samples (grey) with similar initial mass between $500 \mu \mathrm{m}$ and $250 \mu \mathrm{m}$ in size. The sample RN35 is also compared to sample blanks which have very low contamination to illustrate the overall purity of RN35.

\begin{tabular}{|c|c|c|c|c|c|}
\hline Name & $\begin{array}{c}\text { Rock Type/Gransize } \\
(\boldsymbol{\mu m})\end{array}$ & $\begin{array}{c}\text { Avg. } \\
\text { (nAmps) }\end{array}$ & $\begin{array}{c}\text { Total }{ }^{\mathbf{1 0}} \mathbf{B e} \\
\text { counts }\end{array}$ & $\begin{array}{c}\text { Quartz } \\
\text { Mass }\end{array}$ \\
\hline \hline 1 & RN35 & Greywacke/125-63 & 9155 & 1957.000 & 14.2272 \\
\hline 2 & RN60 & Granite/500-250 & 8437.15 & 546 & 10.164 \\
\hline 3 & RN62 & Granite/500-251 & 7443 & 570.000 & 12.890 \\
\hline 4 & RN66 & Granite/500-252 & 9195 & 2602.000 & 17.562 \\
\hline 5 & RN67 & Granite/500-253 & 9038 & 3611.000 & 14.892 \\
\hline 6 & RN33 & Blank & 6634.5 & 55 & N/A \\
\hline 7 & RN45 & Blank & 9613 & 34 & N/A \\
\hline 8 & RN71 & Blank & 9531 & 44.000 & N/A \\
\hline 9 & RN85 & Blank & 7493.69 & 45 & N/A \\
\hline 10 & RN98 & Blank & 7650.98 & 52 & N/A \\
\hline
\end{tabular}




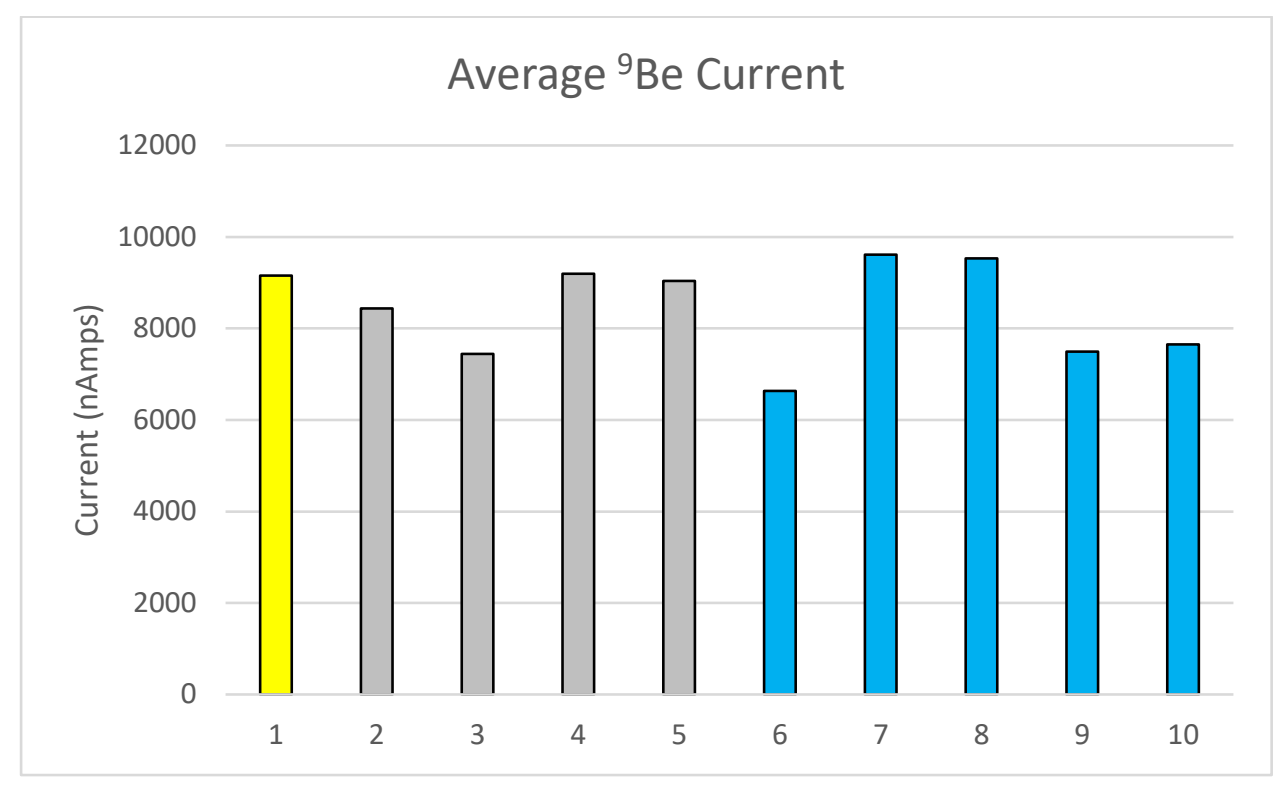

Figure 4.24 ${ }^{9}$ Be current comparison graph

${ }^{9} \mathrm{Be}$ current strength is a function of sample purity. The results from RN35 (yellow) show that this sample and procedure are comparable to standard purification protocols for coarser grained material.

\subsection{Discussion of the new quartz purification procedure}

The framework provided by the new purification protocol shows that fine-grained quartz (125$63 \mu \mathrm{m})$ can be successfully purified to produce reliable results. The success of this procedure means that rock types containing very fine quartz (native grain size between 125-63 $\mu \mathrm{m}$ ) are now fit for terrestrial cosmogenic nuclide studies. Presented below is a discussion of why incomplete purification is an issue, how this method fits into the context with other purification procedures, and what this means for future studies.

\subsubsection{Ramifications of incomplete purification}

Incomplete mineral purification is a critical issue for calculating exposure age, burial ages, and erosion rates (Table 4.8) and significant effort has gone into purification procedures. Incomplete purification can result in erroneously old age estimation, saturation for sample exposure and burial ages and artificially low erosion rates for specific applications, as well as 
sample failure in the lab. Table 4.8 shows the results of incomplete purification procedures for both exposure ages and erosion rates. The table was made by summing the amount of material digested, the measured ${ }^{10} \mathrm{Be} /{ }^{9} \mathrm{Be}$ ratio, and the analytical error starting with the purified sample on the left. The results show $\sim 27 \%$ over estimation in sample exposure age and a $29 \%$ reduction in erosion rate between the clean sample and acid stage 3 (2.5\% HF leach) (Table 4.8). For most applications a $27-29 \%$ error would seriously compromise the results.

\section{Table 4.8 Incomplete purification age and erosion rate calculations}

This table shows the effects of incomplete quartz purification on calculated age. The amount of material digested, ${ }^{10} \mathrm{Be}-{ }^{9} \mathrm{Be}$ ratio, and measurement error are summed from each preceding stage starting on the right with the cleaned sample (HP-16-11/RN35). Apparent exposure ages and erosion rates were calculated using the online exposure age calculator CRONUS-Earth and only the LDSn (Lifton et al., 2014) results are shown.

\begin{tabular}{|c|c|c|c|c|c|c|}
\hline Stage & tage $1 \& 2$ & Stage 3 & Stage $4 \& 5$ & Stage6\&7 & Stage $8 \& 9$ & Cleaned Sample \\
\hline Grams digested & 1.5308 & 3.8818 & 2.6643 & 2.2298 & 1.8166 & 14.2272 \\
\hline Summation $(\mathrm{g})$ & 26.3505 & 24.8197 & 20.9379 & 18.2736 & 16.0438 & 14.2272 \\
\hline${ }^{10} \mathrm{Be} /{ }^{9} \mathrm{Be}$ ratio & ;.16E-11 & 7.04E-14 & $2.66 \mathrm{E}-14$ & $1.89 \mathrm{E}-14$ & $1.57 \mathrm{E}-14$ & $9.59 \mathrm{E}-14$ \\
\hline Summation & i.18E-11 & $2.27 \mathrm{E}-13$ & $1.57 \mathrm{E}-13$ & $1.30 \mathrm{E}-13$ & $1.12 \mathrm{E}-13$ & $9.59 \mathrm{E}-14$ \\
\hline Uncertainty & '.07E-13 & $3.74 \mathrm{E}-15$ & 2.64E-15 & $1.85 \mathrm{E}-15$ & $9.99 \mathrm{E}-16$ & $2.36 \mathrm{E}-15$ \\
\hline Summation & ${ }^{\prime} .18 \mathrm{E}-13$ & $1.16 \mathrm{E}-14$ & $7.84 \mathrm{E}-15$ & $5.20 \mathrm{E}-15$ & $3.35 \mathrm{E}-15$ & $2.36 \mathrm{E}-15$ \\
\hline $\begin{array}{l}\text { LDSn Age } \\
(\mathrm{yr})\end{array}$ & jaturated & 28770 & 23433 & 22311 & 21623 & 21130 \\
\hline $\begin{array}{l}\text { Total Uncertainty } \\
\text { (yr) }\end{array}$ & jaturated & 3799 & 3058 & 2554 & 2161 & 1954 \\
\hline $\begin{array}{l}\text { LDSn Erosion } \\
(\mathrm{m} / \mathrm{Myr})\end{array}$ & iaturated & 23.8 & 29.5 & 31 & 32 & 32.8 \\
\hline $\begin{array}{l}\text { Total Uncertainty } \\
\text { (m/Myr) }\end{array}$ & ;aturated & 3.16 & 3.86 & 3.56 & 3.21 & 3.046 \\
\hline
\end{tabular}

\subsubsection{Conservative quartz purification method comparison}

The conservative purification method outlined above is specifically designed for material between $125 \mu \mathrm{m}$ and $63 \mu \mathrm{m}$, which means that acid etching happens in a controlled manner. This method is more conservative than other purification procedures designed to clean coarser grained quartz.

This deliberate etching method shows that $35 \%$ mass reduction is recommended to purify very fine-grained quartz. This amount of mass loss ensures that large quantities major elements will 
not present an issue during column chemistry or subsequent $\mathrm{BeOH}$ precipitation phase and all meteoric ${ }^{10} \mathrm{Be}$ is removed (Ochs and Ivy-Ochs 1997). This is a slightly elevated value relative to the $\sim 20-30 \%$ mass loss commonly reported (Brown et al., 1991; Klein et al., 1997, Gosse and Phillips, 2001; Corbett et al., 2010), but likely represents a comparable amount of surface thickness removed as other purification procedures for coarser material. The increased required mass loss is in part due to slightly elevated $\mathrm{Al}$ in material smaller than $250 \mu \mathrm{m}$ (Kohl and Nishiizumi, 1992). While the chemical character will vary for every sample, this study provides basic constraints on sample purity for quartz between $125 \mu \mathrm{m}$ and $63 \mu \mathrm{m}$.

For fine-grained samples, carefully decanting supernates (acid or rinse water) across the purification process is critical. It is imperative that fine material suspended in a supernate be retained with the sample. This will reduce unquantifiable mass loss related to decanting supernates and aid in measuring the true mass loss from grain-size reduction.

\subsubsection{The utility of very fine-grained quartz}

As with other studies which explore other mineral/nuclide pair combinations, this work serves to expand the range of rock types which can reliably produce accurate results. It is in the vein of other purification procedures outlined by Blard and others (2008), Marrero and others (2018), Zerathe and others (2017), Eaves and others (2018), Goehring and others (2014), and Caraceddo and others (2018). Each of these studies has provided critical insights to expand the utility of terrestrial cosmogenic nuclide work. This purification procedure enables the investigation of more locations like Victoria Land, New Zealand, and drainage basins with mature very fine sediment. 


\section{MAWSON GLACIER: THINNING THROUGH THE HOLOCENE}

\subsection{Introduction}

\section{$\underline{5.1 .1}$ Context}

The overall goal for this chapter is to constrain the glacial history of an outlet glacier that drains the East Antarctic Ice Sheet through the Scott Coast in southern Victoria Land. Mawson Glacier drains an area of approximately $16,000 \mathrm{~km}^{2}$ and the glacial thinning history of Mawson Glacier is still unresolved. Adjacent outlet glaciers may respond to external forcings independent of one another due to topographic controls and outlet geometry (Felikson et al., 2017). As such, the rapid thinning of Mackay Glacier, $100 \mathrm{~km}$ south of Mawson Glacier, at $\sim 6.5$ kya (Jones et al., 2015) may not reflect the regional response of outlet glaciers in the area (Felikson et al., 2017). In order to better assess the regional ice sheet response to large scale grounding line retreat in the Ross Sea, a second glacier with a different bed geometry is necessary to explore the regional ice sheet history.

The style of deglaciation in the Ross Sea region has implications for the drivers of ice sheet retreat during the Holocene. If the rapid thinning of Mackay Glacier was an isolated event, then other glaciers in Victoria Land may have responded to the same forcing that drove deglaciation in the central Ross Sea during the early Holocene. If the thinning at Mackay Glacier was not an isolated event, then it implies that deglaciation of the western Ross Sea was driven by a different forcing than what drove the deglaciation in the central Ross Sea since the Last Glacial Maximum.

\section{$\underline{5.1 .2}$ Aim of work}

The aim of this work is to reconstruct the Holocene ice-surface-history for Mawson Glacier. 


\subsection{Sampling}

\section{$\underline{5.2 .1}$ Sample collection}

In January 2016 a field sampling campaign was completed at three sites along the margin of the modern day Nordenskjöld Ice Tongue at Mawson Glacier (Figure 5.1). Material was processed from two sample sites, Bruce Point and Mount Murray, located $\sim 10 \mathrm{~km}$ apart. These two nunataks span the length of an over-deepened basin below the Nordenskjöld Ice Tongue. This glacial system provides an ideal natural laboratory to reconstruct the outlet glacier history and understand the fundamental mechanisms that drive and resist thinning. Both collection sites were covered by ice during the Last Glacial Maximum, are adjacent to zones of modern high-velocity ice (Figure 5.2) and proved to be exceptionally productive sampling localities for glacial erratics. Glacially transported samples were collected using an elevation transect oriented perpendicular to the modern ice surface up to $\sim 250 \mathrm{~m}$ and $\sim 160 \mathrm{~m}$ above it at Bruce Point and Mount Murray, respectively. 


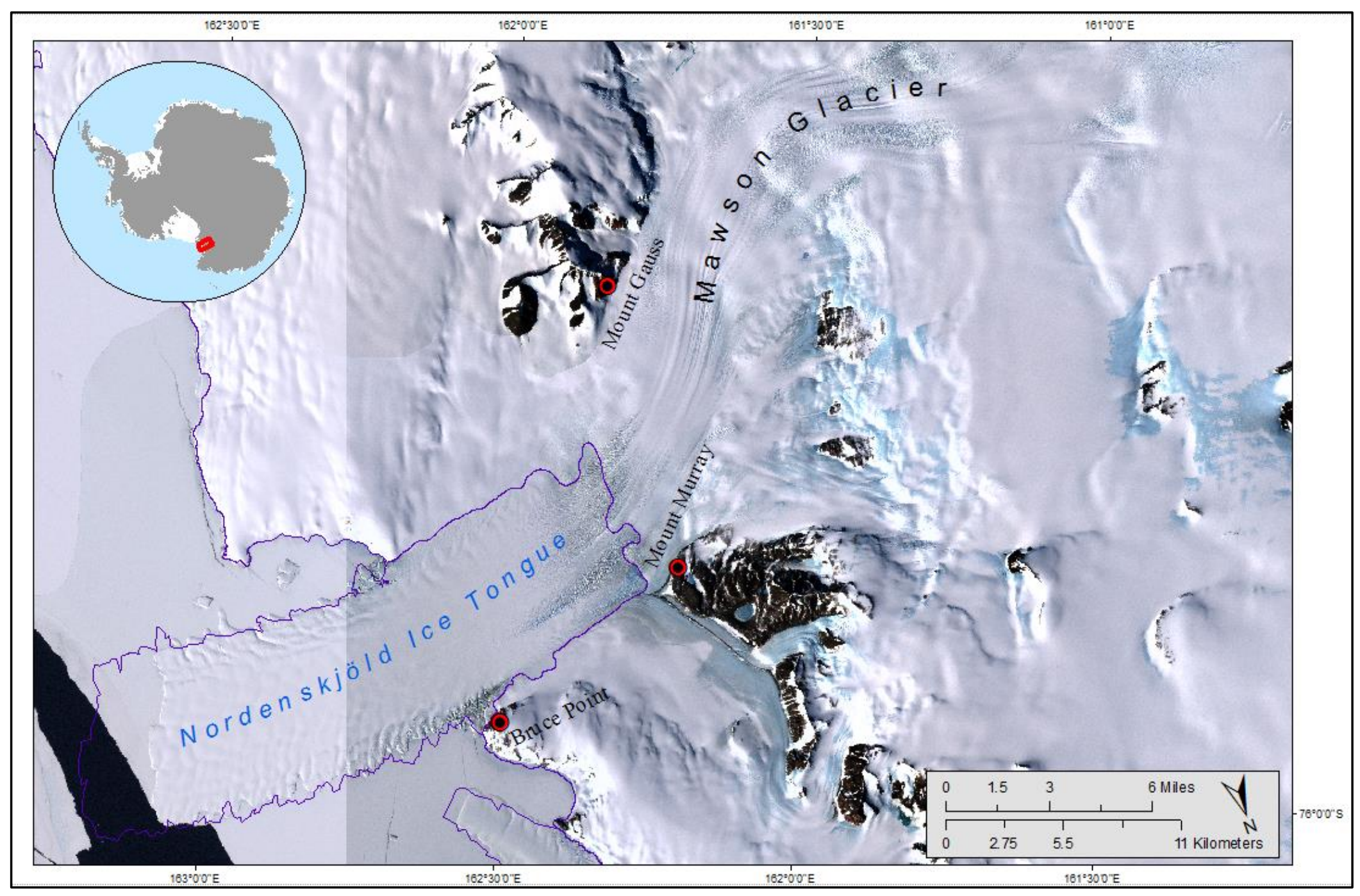

\section{Figure 5.1 Mawson Glacier location map}

Map of the area sampled during the January 2016 campaign. Sample locations are shown with red dots, current grounding line and extent of Nordenskjöld Ice Tongue are shown with a blue line, and dominant features and sample locations are named. 


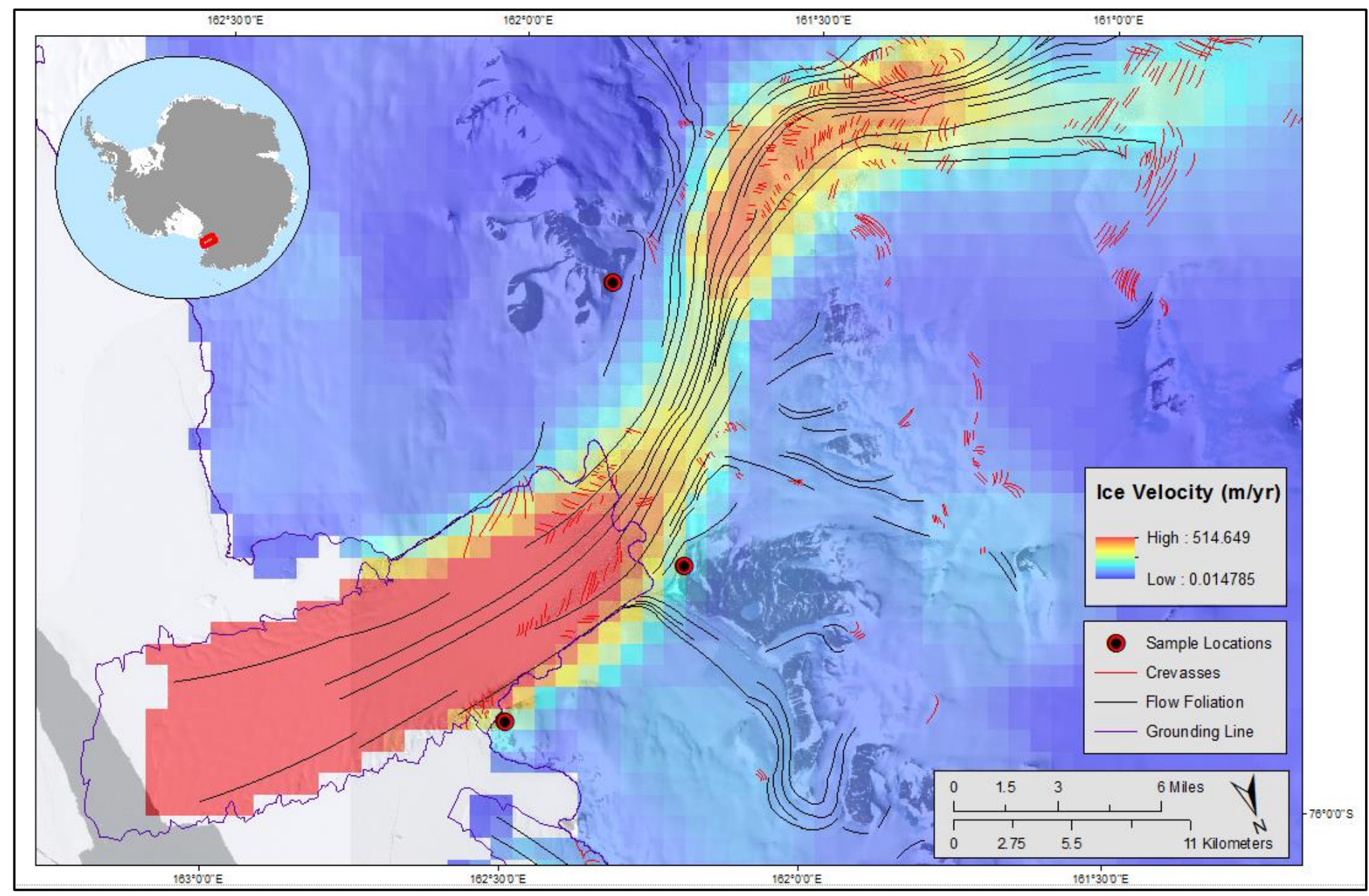

\section{Figure 5.2 Mawson Glacier ice velocity map}

Map showing the relationship of the three primary sample locations to zones of high velocity ice. Current areas of high velocity ice are thought to have also been areas of high-velocity ice in the past and represent ideal targets for sampling because when the glacier was thicker the ice would have been dynamic enough to remove any previously deposited clasts and may have been abrasive enough to remove the top several meters of bedrock to "reset" the terrestrial cosmogenic nuclide clock. Also mapped here are flow foliation (oriented parallel to flow direction) and crevasses (oriented approximately perpendicular of flow direction) which document the flow orientation and state of stress in the glacier. 


\section{$\underline{5.2 .2}$ Bruce Point}

Bruce Point is located $\sim 10 \mathrm{~km}$ seaward of the modern grounding line and adjacent to the Nordenskjöld Ice Tongue (Figure 5.1). It is well positioned in the catchment to record the likely maximum rate of change at Mawson Glacier and constrain the onset of thinning. Bruce Point is a high-relief nunatak with steep stair-stepped flanks. Rock benches present at the site are glacially moulded and provide stable locations for glacial erratics to be deposited. The rock surface is glacially moulded, striated, wind-swept, locally weathered, and devoid of large snow fields. Richard Jones processed 21 quartz-bearing glacial erratics spanning the elevation range from $\sim 20 \mathrm{~m}$ to $\sim 250 \mathrm{~m}$ above the present ice surface. These data are not presented in this thesis, but the chronology will be discussed.

\section{$\underline{5.2 .3}$ Mount Murray}

Mount Murray is located adjacent to the modern-day grounding line (Figure 5.1). This location is ideally positioned in the catchment to track the most recent dynamic changes in ice-surfaceelevation and capture when the grounding line stabilized to its current configuration. Mount Murray consists of a high-relief mountain peak surrounded by an areally extensive, moderaterelief plateau (Figure 5.3B), and is locally fringed by distinct sharp-crested moraine ridges along its north-eastern and southern flanks. The bedrock adjacent to Mawson Glacier is glacially moulded, striated, weathered, wind-swept, and has locally developed perennial snow patches and small non-dynamic glaciers. The bedrock is frost-shattered up to $\sim 160 \mathrm{~m}$ above the current ice surface, but still holds glacial erratics. A composite age-elevation transect was made from rocks collected from sample transects 1, 2 and 4 (Figure 5.3). Sample transect 1 started at $\sim 100 \mathrm{~m}$ and reached $\sim 160 \mathrm{~m}$ above the current ice surface. The landscape around transect 1 was glacially moulded with local block fields and fractured bedrock. The exposed bedrock surfaces had glacial striae and bulleted glacial erratics up to an elevation of $\sim 160 \mathrm{~m}$ 
(Figure 5.5). Sample transects 2 and 4 started at the current ice surface and reached $\sim 40 \mathrm{~m}$ and $\sim 100 \mathrm{~m}$ respectively (Figure 5.6). Both sample transects were made on small ridges oriented perpendicular to the edge of the glacier. The bedrock in the lower part of the transect is glacially moulded and striated, above $\sim 60 \mathrm{~m}$ the bedrock is fractured with erratics continuing up to $100 \mathrm{~m}$ above the ice surface. A total of 21 quartz-bearing glacial erratics were processed along with three boulder tops collected from a moraine ridge adjacent to the glacier. The samples were collected from four different transects which range in elevation from the current ice surface to $\sim 160 \mathrm{~m}$ above the glacier's surface (Figure 5.3A). No material was used from sample transect three. 

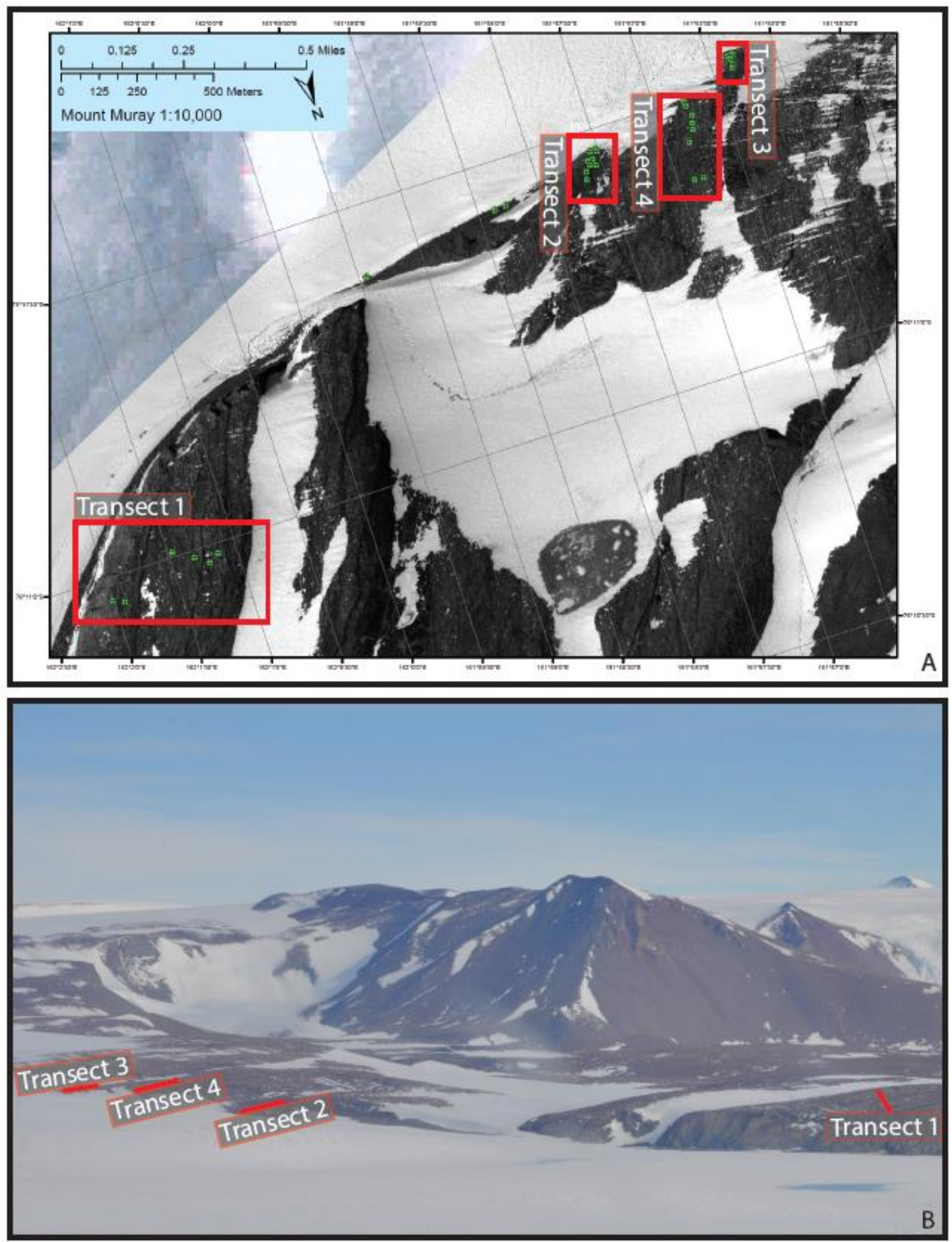

\section{Figure 5.3 Mawson Glacier transect map}

The sample location map (A) and oblique airphoto (B) both show the relative position of the sampling transects used to evaluate the thinning history of Mawson Glacier at Mount Murray. The oblique airphoto also shows the complex topographic nature of Mount Murray. 


\subsubsection{Accelerator mass spectrometry instruments}

Two different accelerator mass spectrometers were used in order to complete this work. A total of 5 samples were run on the 0.5 MV Tandy Accelerator at ETH Zurich and a total of 17 samples were run on the upgraded FN 7 MV tandem AMS at PRIME Lab at Purdue University.

\subsection{Results}

\section{$\underline{5.3 .1} \underline{\text { Lab blanks }}$}

Sample suites were individually blank corrected due to the wide range of blank correction values (Table 5.1). The total ${ }^{10} \mathrm{Be}$ lab blank value changes $\sim 2$ orders of magnitude between RN11, RN71, and RN85 likely because blank RN11 was analysed on the smaller instrument at ETH Zurich. The sample suites associated with lab blank RN71 and RN85 had low mass samples and samples were collected within 1 vertical metre of glacial ice.

\section{$\underline{5.3 .2}$ Mount Murray chronology}

Of the 22 samples processed from Mount Murray (Figures 5.4 and 5.5), 15 are in stratigraphic order and have similar exposure ages (Figure 5.6 and A and B) and 7 samples are out of stratigraphic order and likely have a prior exposure history (Figure 5.6B and C). Outliers were removed from the data set in order to interpret the Holocene thinning history (Figure 5.6C). Please see the outlier's section (5.3.7), below for a detailed explanation of sample omission. Monte Carlo least squares linear regression analysis was used to determine the time-average estimate for thinning at Mount Murray (Figure 5.4E). See the rate, timing, and magnitude of thinning at Mawson Glacier section (5.3.8) for an explanation of the statistical analysis results. 


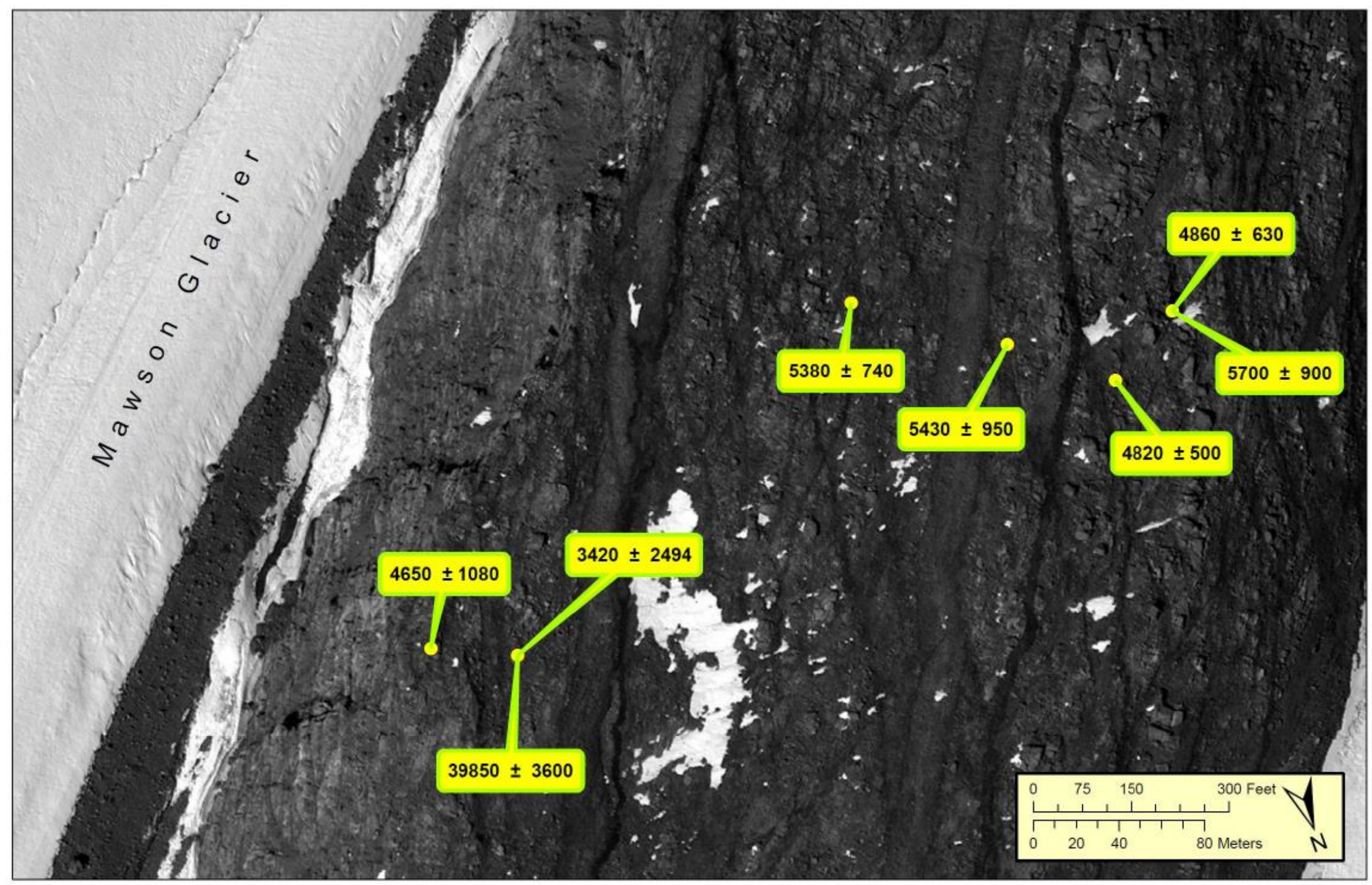

Figure 5.4 Mount Murry's eastern transect

This map shows the position and age of erratics collected on Mount Murray along transect 1 . The elevation ranges from $\sim 100 \mathrm{~m}$ to $\sim 160 \mathrm{~m}$ above the current ice surface 


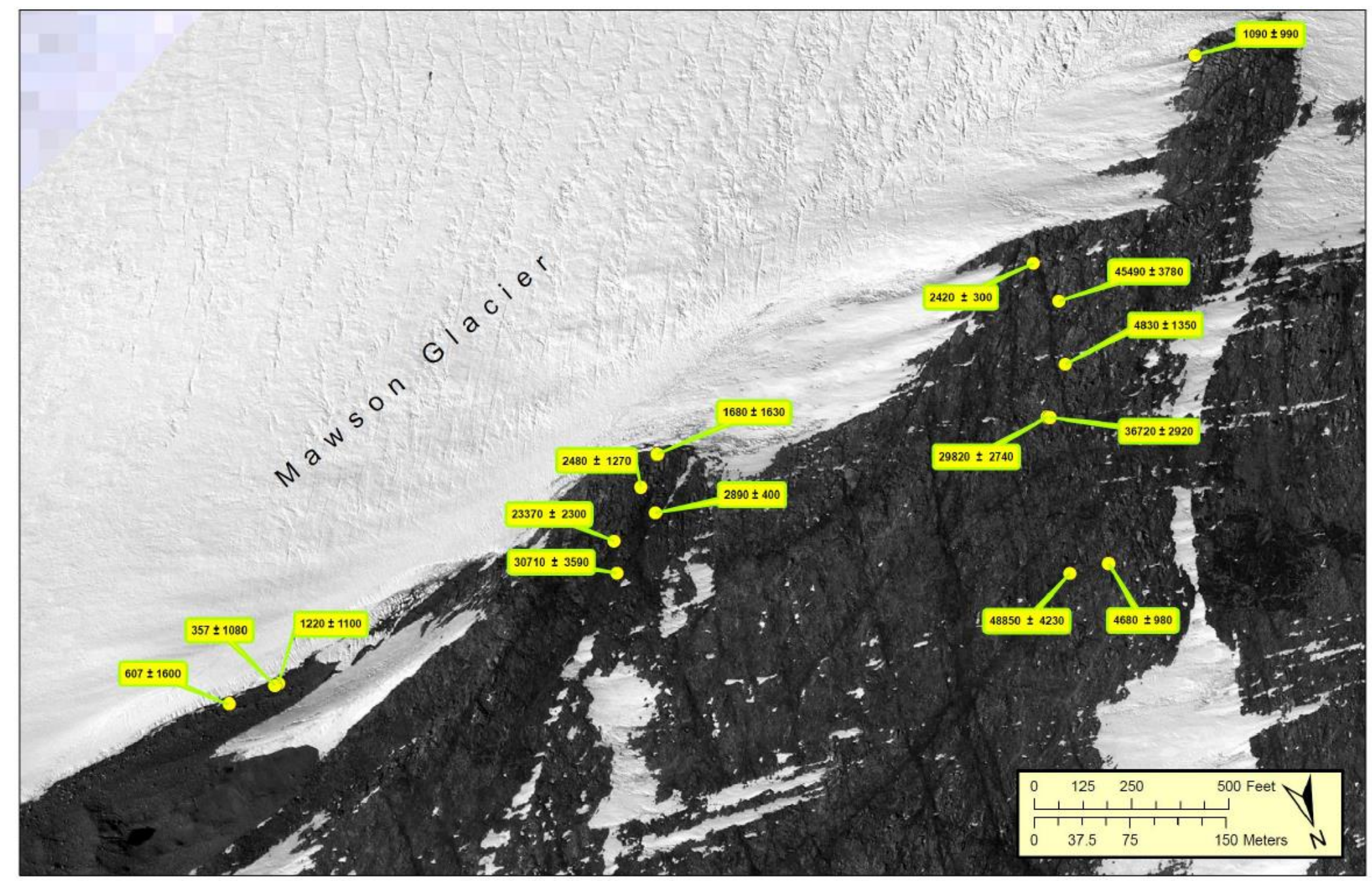

Figure 5.5 Mount Murray's western transects

This map shows the position and age of erratics collected on Mount Murray along transects 2, 3 and 4 (left to right). The elevation ranges from $1 \mathrm{~m}$ to $\sim 100 \mathrm{~m}$ above the local ice surface adjacent to the sample transect 


\subsubsection{Holocene thinning of Mawson Glacier}

The exposure ages at Mount Murray are youngest adjacent to the modern ice surface and become older with increasing elevation. Eight samples record $125 \mathrm{~m}$ of rapid ice-surface-elevation change concentrated between $\sim 5.8$ kya and $\sim 4.7$ kya from $\sim 160 \mathrm{~m}$ to $\sim 30 \mathrm{~m}$ above the current ice surface (Figure $5.4 \mathrm{C}$ ). Three samples record $\sim 15 \mathrm{~m}$ of gradual thinning between $\sim 2.9$ kya to $\sim 2.4$ kya between $\sim 30 \mathrm{~m}$ and $\sim 15 \mathrm{~m}$ above the current ice surface. The remaining two samples were collected $\sim 1 \mathrm{~m}$ above the current ice surface, had low concentrations of ${ }^{10} \mathrm{Be}$. These samples could not be dated, implying a very short exposure history. 
Table 5.1 Sample and blank data: Mawson Glacier

This table contains all relevant data for blank correction. ${ }^{10} \mathrm{Be}$ lab blank and total error for each individual blank The yellow box is the sample blank..

\begin{tabular}{|c|c|c|c|c|c|c|c|c|c|c|c|c|c|}
\hline $\begin{array}{l}\text { Lab } \\
\text { Number }\end{array}$ & ${ }^{10} \mathrm{Be} /{ }^{9} \mathrm{Be}$ & Uncertainty & $\begin{array}{l}\text { Spike } \\
\text { Mass (g) }\end{array}$ & $\begin{array}{l}\text { Spike } \\
\text { Concentration } \\
\text { (ppm) }\end{array}$ & $\begin{array}{l}\text { Uncertainty } \\
\text { (ppm) }\end{array}$ & Spike Be (g) & $\begin{array}{l}\text { Uncertainty } \\
\text { (g) }\end{array}$ & Qtz (g) & $\begin{array}{l}{ }^{10} \text { Be Lab } \\
\text { Blank (at) }\end{array}$ & $\begin{array}{l}\text { Uncertainty } \\
\text { (at) }\end{array}$ & ${ }^{9} \mathrm{Be}$ (at) & ${ }^{10} \mathrm{Be}(\mathrm{at} / \mathrm{g})$ & $\begin{array}{l}\text { Uncertainty } \\
\text { (at/g) }\end{array}$ \\
\hline RN1 & $8.37 \mathrm{E}-14$ & $7.03 \mathrm{E}-15$ & 0.8374 & 305 & 3 & $2.554 \mathrm{E}-04$ & $2.51 \mathrm{E}-06$ & 36.7708 & $6.64 E+04$ & $1.34 \mathrm{E}+04$ & $1.71 \mathrm{E}+19$ & $3.70 E+04$ & $3.30 \mathrm{E}+03$ \\
\hline RN2 & 7.93E-13 & $1.90 \mathrm{E}-14$ & 0.8422 & 305 & 3 & $2.569 \mathrm{E}-04$ & $2.53 \mathrm{E}-06$ & 45.4975 & $6.64 \mathrm{E}+04$ & $1.34 \mathrm{E}+04$ & $1.72 \mathrm{E}+19$ & $2.98 \mathrm{E}+05$ & $7.76 \mathrm{E}+03$ \\
\hline RN4 & $3.75 \mathrm{E}-14$ & $5.81 \mathrm{E}-15$ & 0.8393 & 305 & 3 & $2.560 \mathrm{E}-04$ & $2.52 \mathrm{E}-06$ & 26.5106 & $6.64 E+04$ & $1.34 \mathrm{E}+04$ & $1.71 \mathrm{E}+19$ & $2.17 \mathrm{E}+04$ & $3.79 \mathrm{E}+03$ \\
\hline RN6 & $3.36 \mathrm{E}-13$ & $1.34 \mathrm{E}-14$ & 0.8412 & 305 & 3 & $2.566 \mathrm{E}-04$ & $2.52 \mathrm{E}-06$ & 24.8787 & $6.64 \mathrm{E}+04$ & $1.34 \mathrm{E}+04$ & $1.71 \mathrm{E}+19$ & $2.29 \mathrm{E}+05$ & $9.56 \mathrm{E}+03$ \\
\hline RN9 & $1.19 \mathrm{E}-13$ & $9.72 \mathrm{E}-15$ & 0.8439 & 305 & 3 & $2.574 \mathrm{E}-04$ & $2.53 \mathrm{E}-06$ & 51.8779 & $6.64 \mathrm{E}+04$ & $1.34 \mathrm{E}+04$ & $1.72 \mathrm{E}+19$ & $3.80 \mathrm{E}+04$ & $3.26 \mathrm{E}+03$ \\
\hline RN11 & $1.32 \mathrm{E}-14$ & $2.33 \mathrm{E}-15$ & 0.7159 & 305 & 3 & $2.183 \mathrm{E}-04$ & $2.15 \mathrm{E}-06$ & & $6.64 \mathrm{E}+04$ & $1.34 \mathrm{E}+04$ & $1.46 \mathrm{E}+19$ & & \\
\hline $\begin{array}{l}\text { Lab } \\
\text { Number }\end{array}$ & ${ }^{10} \mathrm{Be} /{ }^{9} \mathrm{Be}$ & Uncertainty & $\begin{array}{l}\text { Spike } \\
\text { Mass (g) } \\
\end{array}$ & $\begin{array}{l}\text { Spike } \\
\text { Concentration } \\
\text { (ppm) }\end{array}$ & $\begin{array}{l}\text { Uncertainty } \\
\text { (ppm) }\end{array}$ & Spike Be (g) & $\begin{array}{l}\text { Uncertainty } \\
\text { (g) }\end{array}$ & Qtz (g) & $\begin{array}{l}{ }^{10} \text { Be Lab } \\
\text { Blank (at) }\end{array}$ & $\begin{array}{l}\text { Uncertainty } \\
\text { (at) }\end{array}$ & ${ }^{9} \mathrm{Be}(\mathrm{at})$ & ${ }^{10} \mathrm{Be}(\mathrm{at} / \mathrm{g})$ & $\begin{array}{l}\text { Uncertainty } \\
\text { (at/g) }\end{array}$ \\
\hline RN60 & $2.32 \mathrm{E}-14$ & $1.07 \mathrm{E}-15$ & 0.8814 & 305 & 3 & $2.688 \mathrm{E}-04$ & $2.64 \mathrm{E}-06$ & 10.164 & $4.05 \mathrm{E}+04$ & $6.13 \mathrm{E}+03$ & $1.80 \mathrm{E}+19$ & 37119 & 2020 \\
\hline RN61 & $6.15 \mathrm{E}-14$ & $1.92 \mathrm{E}-15$ & 0.8834 & 305 & 3 & $2.694 \mathrm{E}-04$ & $2.65 \mathrm{E}-06$ & 28.820 & $4.05 \mathrm{E}+04$ & $6.13 E+03$ & $1.80 \mathrm{E}+19$ & 36988 & 1277 \\
\hline RN62 & $2.74 \mathrm{E}-14$ & $1.19 \mathrm{E}-15$ & 0.8462 & 305 & 3 & $2.581 \mathrm{E}-04$ & $2.54 \mathrm{E}-06$ & 12.890 & $4.05 E+04$ & $6.13 E+03$ & $1.72 \mathrm{E}+19$ & 33539 & 1700 \\
\hline RN63 & 1.67E-14 & $8.75 \mathrm{E}-16$ & 0.8845 & 305 & 3 & $2.698 \mathrm{E}-04$ & $2.65 \mathrm{E}-06$ & 6.535 & $4.05 \mathrm{E}+04$ & $6.13 E+03$ & $1.80 \mathrm{E}+19$ & 39908 & 2628 \\
\hline RN64 & $2.11 \mathrm{E}-13$ & $6.12 \mathrm{E}-15$ & 0.884 & 305 & 3 & $2.696 \mathrm{E}-04$ & $2.65 \mathrm{E}-06$ & 25.133 & $4.05 \mathrm{E}+04$ & $6.13 \mathrm{E}+03$ & $1.80 \mathrm{E}+19$ & 149690 & 4636 \\
\hline RN65 & $3.55 \mathrm{E}-14$ & $1.49 \mathrm{E}-15$ & 0.8855 & 305 & 3 & $2.701 \mathrm{E}-04$ & $2.66 \mathrm{E}-06$ & 36.063 & $4.05 \mathrm{E}+04$ & $6.13 E+03$ & $1.80 \mathrm{E}+19$ & 16649 & 784 \\
\hline RN66 & $2.04 \mathrm{E}-13$ & $4.94 \mathrm{E}-15$ & 0.8811 & 305 & 3 & $2.687 \mathrm{E}-04$ & $2.64 \mathrm{E}-06$ & 17.562 & $4.05 \mathrm{E}+04$ & $6.13 \mathrm{E}+03$ & $1.80 \mathrm{E}+19$ & 206558 & 5468 \\
\hline RN67 & $2.88 \mathrm{E}-13$ & $5.71 \mathrm{E}-15$ & 0.8857 & 305 & 3 & $2.701 \mathrm{E}-04$ & $2.66 \mathrm{E}-06$ & 14.892 & $4.05 \mathrm{E}+04$ & $6.13 \mathrm{E}+03$ & $1.81 \mathrm{E}+19$ & 346813 & 7743 \\
\hline RN68 & $4.76 \mathrm{E}-15$ & $6.80 \mathrm{E}-16$ & 0.8784 & 305 & 3 & $2.679 \mathrm{E}-04$ & $2.64 \mathrm{E}-06$ & 1.691 & $4.05 \mathrm{E}+04$ & $6.13 \mathrm{E}+03$ & $1.79 \mathrm{E}+19$ & 26475 & 8079 \\
\hline RN69 & $1.44 \mathrm{E}-14$ & $8.14 \mathrm{E}-16$ & 0.886 & 305 & 3 & $2.702 \mathrm{E}-04$ & $2.66 \mathrm{E}-06$ & 5.8 & $4.05 \mathrm{E}+04$ & $6.13 E+03$ & $1.81 \mathrm{E}+19$ & 37477 & 2763 \\
\hline $\begin{array}{l}\text { RN71 } \\
\text { (BLANK) }\end{array}$ & $2.25 \mathrm{E}-15$ & $3.40 \mathrm{E}-16$ & 0.8825 & 305 & 3 & $2.692 \mathrm{E}-04$ & 2.65E-06 & & $4.05 \mathrm{E}+04$ & 6126.411775 & $1.80 \mathrm{E}+19$ & & \\
\hline $\begin{array}{l}\text { Lab } \\
\text { Number }\end{array}$ & ${ }^{10} \mathrm{Be} /{ }^{9} \mathrm{Be}$ & Uncertainty & $\begin{array}{l}\text { Spike } \\
\text { Mass (g) }\end{array}$ & $\begin{array}{l}\text { Spike } \\
\text { Concentration } \\
\text { (ppm) }\end{array}$ & $\begin{array}{l}\text { Uncertainty } \\
\text { (ppm) }\end{array}$ & Spike Be () & $\begin{array}{l}\text { Uncertainty } \\
\text { (g) }\end{array}$ & Qtz (g) & $\begin{array}{l}{ }^{10} \text { Be Lab } \\
\text { Blank (at) }\end{array}$ & $\begin{array}{l}\text { Uncertainty } \\
\text { (at) }\end{array}$ & ${ }^{9} \mathrm{Be}$ (at) & ${ }^{10} \mathrm{Be}(\mathrm{at} / \mathrm{g})$ & $\begin{array}{l}\text { Uncertainty } \\
\text { (at/g) }\end{array}$ \\
\hline RN79 & $4.02 \mathrm{E}-14$ & $1.82 \mathrm{E}-15$ & 0.8851 & 305 & 3 & $2.700 \mathrm{E}-04$ & $2.66 \mathrm{E}-06$ & 34.2475 & $6.63 \mathrm{E}+04$ & $1.34 \mathrm{E}+04$ & $1.80 \mathrm{E}+19$ & $1.92 \mathrm{E}+04$ & $1.06 \mathrm{E}+03$ \\
\hline RN80 & $2.46 \mathrm{E}-15$ & 4.10E-16 & 0.8832 & 305 & 3 & 2.694E-04 & 2.65E-06 & 2.3958 & $6.63 \mathrm{E}+04$ & $1.34 \mathrm{E}+04$ & $1.80 \mathrm{E}+19$ & $-9.21 \mathrm{E}+03$ & $6.37 \mathrm{E}+03$ \\
\hline RN81 & $2.37 \mathrm{E}-15$ & $4.27 \mathrm{E}-16$ & 0.8831 & 305 & 3 & $2.693 \mathrm{E}-04$ & $2.65 \mathrm{E}-06$ & 3.7193 & $6.63 E+04$ & $1.34 \mathrm{E}+04$ & $1.80 \mathrm{E}+19$ & $-6.34 \mathrm{E}+03$ & $4.14 \mathrm{E}+03$ \\
\hline RN82 & $5.30 \mathrm{E}-13$ & $9.02 \mathrm{E}-15$ & 0.8826 & 305 & 3 & $2.692 \mathrm{E}-04$ & $2.65 \mathrm{E}-06$ & 30.0417 & $6.63 \mathrm{E}+04$ & $1.34 \mathrm{E}+04$ & $1.80 \mathrm{E}+19$ & $3.15 \mathrm{E}+05$ & $6.25 \mathrm{E}+03$ \\
\hline RN83 & $1.49 \mathrm{E}-14$ & $1.13 \mathrm{E}-15$ & 0.8846 & 305 & 3 & $2.698 \mathrm{E}-04$ & $2.65 \mathrm{E}-06$ & 5.8945 & $6.63 \mathrm{E}+04$ & $1.34 \mathrm{E}+04$ & $1.80 \mathrm{E}+19$ & $3.43 \mathrm{E}+04$ & $4.17 \mathrm{E}+03$ \\
\hline RN84 & $6.54 \mathrm{E}-13$ & $9.12 \mathrm{E}-15$ & 0.8871 & 305 & 3 & $2.706 \mathrm{E}-04$ & $2.66 \mathrm{E}-06$ & 46.9949 & $6.63 E+04$ & $1.34 \mathrm{E}+04$ & $1.81 \mathrm{E}+19$ & $2.50 \mathrm{E}+05$ & $4.30 \mathrm{E}+03$ \\
\hline $\begin{array}{l}\text { RN } 85 \\
\text { (BLANK) }\end{array}$ & $3.68 \mathrm{E}-15$ & $7.42 \mathrm{E}-16$ & 0.8825 & 305 & 3 & 2.692E-04 & 2.65E-06 & & $6.63 E+04$ & 13357.1219 & $1.80 \mathrm{E}+19$ & & \\
\hline
\end{tabular}




\section{Table 5.2 Mawson Glacier exposure ages}

This table contains all relevant information to calculate ${ }^{10} \mathrm{Be}$ ages and has LDSn age and error highlighted in blue.

\begin{tabular}{|c|c|c|c|c|c|c|c|c|c|c|c|c|c|c|c|}
\hline $\begin{array}{l}\text { Lab } \\
\text { Number }\end{array}$ & $\begin{array}{l}\text { Field } \\
\text { Number }\end{array}$ & Latitude (DD) & Longitude (DD) & $\begin{array}{l}\text { Elevation } \\
(\mathrm{m})\end{array}$ & ATM & $\begin{array}{l}\text { Thickness } \\
(\mathrm{cm})\end{array}$ & $\begin{array}{l}\text { Density } \\
\left(\mathrm{g} /{ }^{3} \mathrm{~cm}\right)\end{array}$ & Shielding & $\begin{array}{l}\text { Errosion } \\
\text { Rate } \\
\text { (m/Myr) }\end{array}$ & $\begin{array}{l}10 \mathrm{Be} \\
\text { Concentration } \\
\text { (at/g) }\end{array}$ & $\begin{array}{l}{ }^{10} \mathrm{Be} \\
\text { Uncertainty } \\
\text { (at) }\end{array}$ & $\begin{array}{l}{ }^{10} \mathrm{Be} \\
\text { Standard }\end{array}$ & $\begin{array}{l}\text { LSDn } \\
\text { Age } \\
\text { (yr) }\end{array}$ & $\begin{array}{l}\text { Internal } \\
\text { Uncertainty }\end{array}$ & $\begin{array}{l}\text { External } \\
\text { Uncertainty }\end{array}$ \\
\hline RN1 & MM-01 & -76.18348 & 162.02873 & 210.7 & ant & 1.9 & 2.7 & 0.9998 & 0 & $3.70 \mathrm{E}+04$ & $3.30 \mathrm{E}+03$ & $\begin{array}{l}07 \text { KNSTD } \\
\end{array}$ & 5533 & 494 & 593 \\
\hline RN2 & MM-02 & -76.18335 & 162.02745 & 214.4 & ant & 2.1 & 2.7 & 0.9994 & 0 & $2.98 \mathrm{E}+05$ & $7.76 \mathrm{E}+03$ & 07KNSTD & 44878 & 1184 & 2928 \\
\hline RN4 & MM-12 & -76.19163531 & 161.9546777 & 212.5 & ant & 5.9 & 2.7 & 0.9934 & 0 & $2.17 \mathrm{E}+04$ & $3.79 \mathrm{E}+03$ & 07KNSTD & 3310 & 579 & 611 \\
\hline RN6 & MM-16 & -76.19110731 & 161.956141 & 230.2 & ant & 4.4 & 2.7 & 0.997 & 0 & $2.29 \mathrm{E}+05$ & $9.56 \mathrm{E}+03$ & 07KNSTD & 34609 & 1457 & 2523 \\
\hline RN9 & MM-29 & -76.1601038 & 161.942187 & 298 & ant & 7.2 & 2.7 & 0.9988 & 0 & $3.80 \mathrm{E}+04$ & $3.26 \mathrm{E}+03$ & 07KNSTD & 5456 & 468 & 568 \\
\hline
\end{tabular}

\begin{tabular}{|c|c|c|c|c|c|c|c|c|c|c|c|c|c|c|c|}
\hline $\begin{array}{l}\text { Lab } \\
\text { Number }\end{array}$ & $\begin{array}{l}\text { Field } \\
\text { Number }\end{array}$ & Latitude (DD) & Longitude (DD) & $\begin{array}{l}\text { Elevation } \\
\text { (m) }\end{array}$ & ATM & $\begin{array}{l}\text { Thickness } \\
(\mathrm{cm})\end{array}$ & $\begin{array}{l}\text { Density } \\
\left(\mathrm{g} /{ }^{3} \mathrm{~cm}\right)\end{array}$ & Shielding & $\begin{array}{l}\text { Errosion } \\
\text { Rate } \\
\text { (m/Myr) }\end{array}$ & $\begin{array}{l}10 \mathrm{Be} \\
\text { Concentration } \\
\text { (at/g) }\end{array}$ & $\begin{array}{l}{ }^{10} \mathrm{Be} \\
\text { Uncertainty } \\
\text { (at) }\end{array}$ & $\begin{array}{l}{ }^{10} \mathrm{Be} \\
\text { Standard }\end{array}$ & $\begin{array}{l}\text { LSDn } \\
\text { Age } \\
\text { (yr) }\end{array}$ & $\begin{array}{l}\text { Internal } \\
\text { Uncertainty }\end{array}$ & $\begin{array}{l}\text { External } \\
\text { Uncertainty }\end{array}$ \\
\hline RN60 & MM-04 & -76.18433 & 162.01981 & 224 & ant & 1.5 & 2.7 & 0.999448 & 0 & $3.71 \mathrm{E}+04$ & $2.02 \mathrm{E}+03$ & 07KNSTD & 5459 & 298 & 439 \\
\hline RN61 & MM-06 & -76.18366816 & 162.0157933 & 369 & ant & 4.9 & 2.7 & 0.999026 & 0 & $3.70 \mathrm{E}+04$ & $1.28 \mathrm{E}+03$ & 07KNSTD & 4856 & 168 & 333 \\
\hline RN62 & MM-07 & -76.18388023 & 162.014447 & 266 & ant & 6.6 & 2.7 & 0.99992 & 0 & $3.35 \mathrm{E}+04$ & $1.70 \mathrm{E}+03$ & 07KNSTD & 4928 & 250 & 384 \\
\hline RN63 & MM-08 & -76.18388023 & 162.014447 & 266 & ant & 5.9 & 2.7 & 0.99992 & 0 & $3.99 \mathrm{E}+04$ & $2.63 \mathrm{E}+03$ & 07KNSTD & 5833 & 385 & 517 \\
\hline RN64 & MM-15 & -76.19132884 & 161.9559299 & 225 & ant & 7.9 & 2.7 & 0.993144 & 0 & $1.50 \mathrm{E}+05$ & $4.64 \mathrm{E}+03$ & 07KNSTD & 23457 & 730 & 1572 \\
\hline RN65 & MM-23 & -76.1923013 & 161.941493 & 252 & ant & 6.9 & 2.7 & 0.991962 & 0 & $1.66 \mathrm{E}+04$ & $7.84 \mathrm{E}+02$ & 07KNSTD & 2438 & 115 & 184 \\
\hline RN66 & MM-28 & -76.19123 & 161.94259 & 278 & ant & 4.3 & 2.7 & 0.99185 & 0 & $2.07 \mathrm{E}+05$ & $5.47 \mathrm{E}+03$ & 07KNSTD & 29933 & 797 & 1950 \\
\hline RN67 & MM-30 & -76.1901245 & 161.94341 & 298 & ant & 3.9 & 2.7 & 0.999691 & 0 & $3.47 \mathrm{E}+05$ & $7.74 \mathrm{E}+03$ & 07KNSTD & 48931 & 1105 & 3124 \\
\hline RN68 & MM-03 & -76.18335 & 162.02745 & 224 & ant & 4.7 & 2.7 & 0.99939 & 0 & $2.65 \mathrm{E}+04$ & $8.08 \mathrm{E}+03$ & 07KNSTD & 3973 & 1213 & 1235 \\
\hline RN69 & MM-05 & -76.18396692 & 162.0174293 & 262 & ant & 7.4 & 2.7 & 0.998032 & 0 & $3.75 \mathrm{E}+04$ & $2.76 \mathrm{E}+03$ & 07KNSTD & 5582 & 411 & 527 \\
\hline
\end{tabular}

\begin{tabular}{|c|c|c|c|c|c|c|c|c|c|c|c|c|c|c|c|}
\hline $\begin{array}{l}\text { Lab } \\
\text { Number }\end{array}$ & $\begin{array}{l}\text { Field } \\
\text { Number }\end{array}$ & Latitude (DD) & Longitude (DD) & $\begin{array}{l}\text { Elevation } \\
(\mathrm{m})\end{array}$ & ATM & $\begin{array}{l}\text { Thickness } \\
(\mathrm{cm})\end{array}$ & $\begin{array}{l}\text { Density } \\
\left(\mathrm{g} /{ }^{3} \mathrm{~cm}\right)\end{array}$ & Shielding & $\begin{array}{l}\text { Errosion } \\
\text { Rate } \\
\text { (m/Myr) }\end{array}$ & $\begin{array}{l}10 \mathrm{Be} \\
\text { Concentration } \\
\text { (at/g) }\end{array}$ & $\begin{array}{l}{ }^{10} \mathrm{Be} \\
\text { Uncertainty } \\
\text { (at) }\end{array}$ & $\begin{array}{l}{ }^{10} \mathrm{Be} \\
\text { Standard }\end{array}$ & $\begin{array}{l}\text { LSDn } \\
\text { Age } \\
\text { (yr) }\end{array}$ & $\begin{array}{l}\text { Internal } \\
\text { Uncertainty }\end{array}$ & $\begin{array}{l}\text { External } \\
\text { Uncertainty }\end{array}$ \\
\hline RN79 & MM-09 & -76.19123 & 161.94259 & 221 & ant & 7.1 & 2.7 & 0.99185 & 0 & $1.92 \mathrm{E}+04$ & $1.06 \mathrm{E}+03$ & 07KNSTD & 2927 & 162 & 237 \\
\hline RN80 & $\mathrm{MM}-10$ & -76.1901245 & 161.94341 & 204 & ant & 4.4 & 2.7 & 0.999691 & 0 & $-9.21 \mathrm{E}+03$ & $6.37 E+03$ & $\begin{array}{l}07 \mathrm{KNSTD} \\
\end{array}$ & $\ldots$ & $-\cdots$ & $-\ldots$ \\
\hline RN81 & MM-17 & -76.18335 & 162.02745 & 242 & ant & 3.5 & 2.7 & 0.99939 & 0 & $-6.34 \mathrm{E}+03$ & $4.14 \mathrm{E}+03$ & 07KNSTD & $\cdots$ & $\cdots$ & $\cdots$ \\
\hline RN82 & MM-24 & -76.18396692 & 162.0174293 & 259 & ant & 2.2 & 2.7 & 0.998032 & 0 & $3.15 \mathrm{E}+05$ & $6.25 \mathrm{E}+03$ & 07KNSTD & 45546 & 914 & 2868 \\
\hline RN83 & MM-26 & -76.18396692 & 162.0174293 & 271 & ant & 7.5 & 2.7 & 0.998032 & 0 & $3.43 \mathrm{E}+04$ & $4.17 E+03$ & $\begin{array}{l}\text { 07KNSTD } \\
\end{array}$ & 5073 & 618 & 686 \\
\hline RN84 & MM-27 & -76.18396692 & 162.0174293 & 278 & ant & 6.7 & 2.7 & 0.998032 & 0 & $2.50 \mathrm{E}+05$ & $4.30 \mathrm{E}+03$ & 07KNSTD & 36721 & 637 & 2278 \\
\hline
\end{tabular}




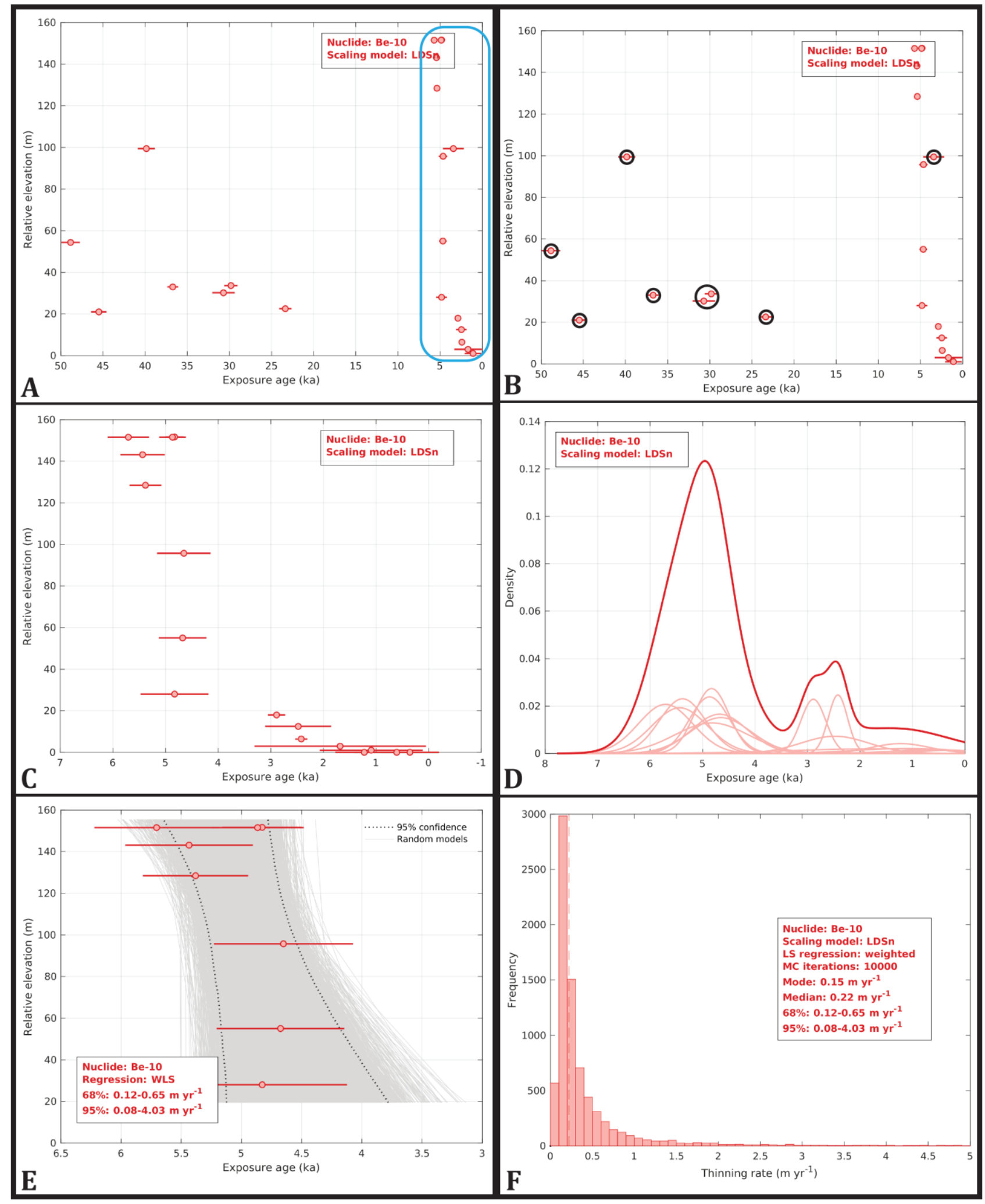

Figure 5.6 Mawson age elevation plots

A) Age elevation plot showing ages for all samples processed from Mount Murray transects. B) All outliers are circled in black and removed from the dataset. The $\sim 5.8$ kya and younger ages shown in the blue box and represent the most recent thinning profile from Mount Murry. C) Enlarged age-elevation profile $~ 5.8$ kya to recent (data in the blue box in A), D) is a 10,000 iteration Monte Carlo linear regression analysis the dotted line shows $95 \%$ confidence interval, grey lines are model runs. The estimated thinning rate is $0.08-4.03 \mathrm{~m} \mathrm{yr}^{-1} 95 \%$ confidence. 


\section{$\underline{\text { 5.3.4 }}$ Sample outliers}

Based on objective criteria a total of eight samples were removed from the Mount Murray dataset as outliers and, as a result, it clarifies the Holocene to recent thinning profile. The samples removed from the dataset are listed in table 5.3 and shown in figures 5.6B and 5.4C. Seven samples were removed from the data set because the glacier geometry, age, position, and morphology suggest one of three potential glaciological depositional scenarios: 1) the samples were previously exposed farther up the glacier and were then re-entrained and deposited at Mount Murray, 2) they were deposited at Mount Murray during a prior glaciation where they experienced repeated cycles of nonerosive cold-based glacial ice cover, melting, and exposure to open sky, or 3) they were supraglacially exposed in a long-lived ablative blueice area where constant accumulation of ${ }^{10} \mathrm{Be}$ would produce an exceptionally old age. One sample was removed from the dataset because of an insufficient amount of quartz yielded an exceedingly large error ( 2 kya). 


\section{Table 5.3 Mount Murray outlier data}

This table contains all the samples removed as outliers from the Mount Murray dataset.

\begin{tabular}{|c|c|c|c|c|c|c|c|c|c|c|c|c|c|c|}
\hline $\begin{array}{l}\text { Field } \\
\text { Number } \\
\end{array}$ & $\begin{array}{l}\text { Latitude } \\
\text { (DD) } \\
\end{array}$ & $\begin{array}{l}\text { Longitude } \\
\text { (DD) }\end{array}$ & $\begin{array}{l}\text { Elevation } \\
(\mathrm{m})\end{array}$ & ATM & $\begin{array}{l}\text { Thickness } \\
(\mathrm{cm})\end{array}$ & $\begin{array}{l}\text { Density } \\
\left(\mathrm{g} /{ }^{3} \mathrm{~cm}\right)\end{array}$ & Shielding & $\begin{array}{l}\text { Errosion } \\
\text { Rate } \\
(\mathrm{m} / \mathrm{Myr}) \\
\end{array}$ & $\begin{array}{l}10 \mathrm{Be} \\
\text { Concentration } \\
\text { (at/g) }\end{array}$ & $\begin{array}{l}{ }^{10} \text { Be } \\
\text { Uncertainty } \\
\text { (at) }\end{array}$ & $\begin{array}{l}{ }^{10} \mathrm{Be} \\
\text { Standard }\end{array}$ & $\begin{array}{l}\text { LSDn Age } \\
(\mathrm{yr})^{\circ}\end{array}$ & $\begin{array}{l}\text { Internal } \\
\text { Uncertainty }\end{array}$ & $\begin{array}{l}\text { External } \\
\text { Uncertainty }\end{array}$ \\
\hline MM-02 & -76.18335 & 162.02745 & 214.4 & ant & 2.1 & 2.7 & 0.9994 & 0 & $2.98 \mathrm{E}+05$ & $7.76 \mathrm{E}+03$ & 07KNSTD & 44878 & 1184 & 2928 \\
\hline $\mathrm{MM}-16$ & 76.1911073 & 161.956141 & 230.2 & ant & 4.4 & 2.7 & 0.997 & 0 & $2.29 \mathrm{E}+05$ & $9.56 \mathrm{E}+03$ & 07KNSTD & 34609 & 1457 & 2523 \\
\hline MM-15 & 76.1913288 & 161.95593 & 225 & ant & 7.9 & 2.7 & 0.993144 & 0 & $1.50 E+05$ & $4.64 E+03$ & 07KNSTD & 23457 & 730 & 1572 \\
\hline MM-28 & -76.19123 & 161.94259 & 278 & ant & 4.3 & 2.7 & 0.99185 & 0 & $2.07 E+05$ & $5.47 \mathrm{E}+03$ & 07KNSTD & 29933 & 797 & 1950 \\
\hline MM-30 & 76.1901245 & 161.94341 & 298 & ant & 3.9 & 2.7 & 0.999691 & 0 & $3.47 \mathrm{E}+05$ & $7.74 \mathrm{E}+03$ & 07KNSTD & 48931 & 1105 & 3124 \\
\hline MM-03 & -76.18335 & 162.02745 & 224 & ant & 4.7 & 2.7 & 0.99939 & 0 & $2.65 \mathrm{E}+04$ & $8.08 \mathrm{E}+03$ & 07KNSTD & 3973 & 1213 & 1235 \\
\hline MM-24 & 76.1839669 & 162.017429 & 259 & ant & 2.2 & 2.7 & 0.998032 & 0 & $3.15 E+05$ & $6.25 \mathrm{E}+03$ & 07KNSTD & 45546 & 914 & 2868 \\
\hline MM-27 & 76.1839669 & 162.017429 & 278 & ant & 6.7 & 2.7 & 0.998032 & 0 & $2.50 \mathrm{E}+05$ & $4.30 \mathrm{E}+03$ & 07KNSTD & 36721 & 637 & 2278 \\
\hline
\end{tabular}




\subsubsection{Rate, timing and magnitude of thinning at Mawson Glacier}

The transect from current ice surface to $\sim 160 \mathrm{~m}$ at Mount Murray records the last $5.8 \mathrm{ky}$ of thinning of Mawson Glacier. The chronology shows continuous rapid thinning from $\sim 5.8$ kya to $\sim 4.9$ kya ( $\sim 130 \mathrm{~m}$ of thinning), gradual thinning from $\sim 4.9$ kya to $\sim 1.5 \mathrm{kya}(15 \mathrm{~m}$ of thinning), and a relatively stable ice-surface-elevation from $\sim 1.5$ kya to the present. A 10,000 iteration Monte Carlo linear regression analysis was completed on samples dating to the main phase of thinning from $\sim 5.8$ kya to $\sim 4.9$ kya to statistically evaluate the dataset for likely maximum thinning rate and to identify outlying samples (Figure 5.6E). The rate of thinning at Mount Murray from $\sim 5.8$ kya to $\sim 4.9 \mathrm{kya}$ is $8 \mathrm{~cm} \mathrm{yr}^{-1}$ to $403 \mathrm{~cm} \mathrm{yr}^{-1}$ with the average best-fit of $22 \mathrm{~cm} \mathrm{yr}^{-1}$ (Figure 5.6F).

\subsection{Discussion of Mawson Glacier's thinning history}

The rapid thinning chronology from 5.8 kya to 4.9 kya at Mount Murray is interpreted to record ice-surface-elevation changes resulting from grounding line migration through the over-deepened basin below the Nordenskjöld Ice Tongue. This ice surface reconstruction may be strongly correlated with grounding-line retreat and subsequent stabilization of Mawson Glacier to its current location proximal to Mount Murray. The Mount Murray thinning history complements the work done at Bruce Point by extending the 5.8 kya and younger thinning history at Mawson Glacier (Jones, Pers. Com.). By moving $10 \mathrm{~km}$ landward, at Mawson Glacier, the Mount Murray chronology fills a sampling gap from the modern ice surface up to $30 \mathrm{~m}$ above ice at Bruce Point. The Mount Murray transect provides ages from 5.8 kya to the present and provides continuous age-elevation control from $1 \mathrm{~m}$ to $\sim 160 \mathrm{~m}$ above current ice surface. The thinning rate found at Mount Murray is between $0.08 \mathrm{~m} / \mathrm{yr}$ to $4.03 \mathrm{~m} / \mathrm{yr}$ and is higher than the $0.14 \mathrm{~m} / \mathrm{yr}$ to $2.76 \mathrm{~m} / \mathrm{yr}$ thinning rate found at Bruce Point. The increase in 
thinning rate at Mount Murray is interpreted to record the final retreat of Mawson Glacier into the over deepened trough below the current ice tongue. More broadly this transect fills crucial spatial and temporal gaps missing from many other terrestrial cosmogenic chronologies along the Scott Coast.

\subsubsection{Glacial History of the Southwestern Ross Sea}

This section places the 5 kya and 6.5 kya thinning profiles collected at Mawson Glacier in the context of other records covering this time period from other parts of the Scott Coast. Multiple data types are used to tie the terrestrial chronologies to marine features along the Scott Coast and offshore in McMurdo Sound (Figure 5.7). Tying terrestrial and marine records together strengthens the current understanding of the glaciological and geomorphic development of the western Ross Sea during the Holocene (Figure 5.8).

Bathymetric mapping of glacial landforms in the southwestern Ross Sea provides critical insight to the paleoglacial position of outlet glaciers along the Victoria Land coast (Halberstadt et al., 2016; Lee et al., 2017; Greenwood et al., 2018). Megascale glacial lineations and grounding zone wedges document the paleoflow path and the stability and retreat of Mawson Glacier (Figure 5.8). When Mawson Glacier is in an advanced configuration it coalesces with ice from an advanced Mackay Glacier with the ice merging north of Beaufort Islands. The megascale glacial lineations join with others in the Central Basin and advance to the Joides Basin (Halberstadt et al., 2016; Lee et al., 2017). There are several sediment cores which constrain the timing of grounding line retreat outboard of the current Mawson and Mackay glacial configurations (Parker, 2017). 


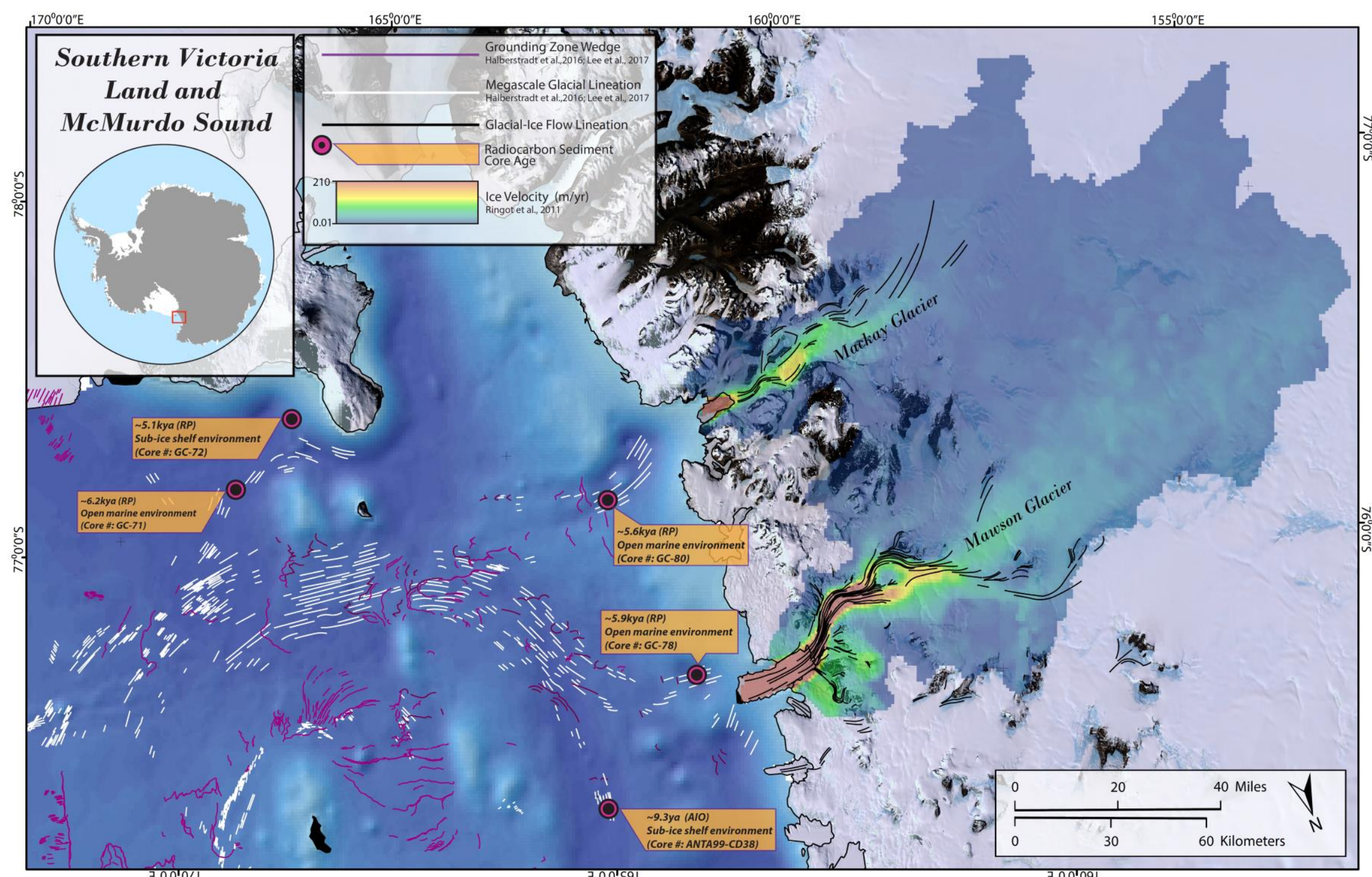

Figure 5.7 Mawson and Mackay glaciers

The map shows the current and paleoglacial configurations of Mawson and Mackay glaciers. The marine landforms and sediment cores record the former flow directions, spatial extents and retreat histories of these two glaciers. Sediment cores also provide temporal constrains on the timing of facies transitions from grounded ice, ice shelf and open water conditions, while the terrestrial data constrains the onshore thinning history. 


\subsubsection{Timing and style of grounding line retreat from offshore records}

This section will discuss the relationship between two sediment cores (GC-78 and GC-80) (Parker et al., 2017), several terrestrial radiocarbon ages (Hall et al., 2004), and the 5.8 kya thinning event that I have identified at Mount Murray. Each data set provides specific context to the history of the Scott Coast between Mawson and Mackay glaciers.

Both GC-78 and GC-80 shows an abrupt facies transition from diamict directly to diatomaceous sediment at $\sim 5.9$ kya and $\sim 5.6$ kya respectively (Figure 5.7). The facies transitions seen in these two sediment cores are interpreted to represent rapid changes in depositional environment from sub-glacial to open marine, with no transitional sub-ice shelf phase (Parker, 2017). Parker interprets this abrupt facies transition as a rapid collapse of grounded ice through two deep basins off the Scott Coast and proposes the driving mechanism as a poleward shift in the westerly winds during the Holocene which enhanced circumpolardeep-water incursion on to the continental shelf and initiated marine-ice-sheet-instability in the southwestern Ross Sea.

Raised beaches occur in several ice-free areas along the Scott Coast. Dating the oldest organic material found in the deposits provides insight into the ice-load history for the Scott Coast. The oldest material found in the deposits is $\sim 6.6$ kya at Kolich Point (Hall et al., 2000) and is interpreted to represent the onset of deglaciation in the southwestern Ross Sea, which agrees well with the new chronology from Bruce Point. Stranded beaches are the result of isostatic uplift following deglaciation. The oldest material collected from these sites represents two possibilities either, they are the minimum age of deglaciation for each individual site, or they are a recolonization age; recolonization may not have occurred immediately after deglaciation. If it is the latter, then the ages may be substantially younger then the age of deglaciation. 
The thinning records from Mawson Glacier are consistent with age, rate, and magnitude of thinning from two other outlet glaciers along the Scott Coast, 100 km south at Mackay Glacier (Figure 5.8) and $\sim 100 \mathrm{~km}$ to the north at David Glacier (Jones et al. 2015, Stutz, Pers. Com; Jones, Pers. Comm.). All three outlet glaciers show evidence of rapid thinning at 6.5 kya. This supports a grounded ice unloading event in the western Ross Sea along the Scott Coast at $\sim 6.6$ kya (Hall et al., 2004). This was likely followed by a piecemeal failure of the subsequent ice shelf between 5.7 kya to 3 kya (Denton and Hall 2000; Hall et al., 2004; Jackson et al. 2018, and Parker, 2017).

\subsubsection{Scott Coast history from local last glacial maximum}

Slightly older multiphase outlet glacier thinning is documented $200 \mathrm{~km}$ south of Mawson Glacier in the area around Koetillitz Glacier (Anderson et al. 2017; Jackson et al., 2018). The thinning record from Koetillitz Glacier extends the overall Scott Coast deglaciation chronology back to the local Last Glacial Maximum at 18 kya (Hall et al., 2015, Anderson et al., 2017; Jackson et al., 2018). The presence of floating ice in McMurdo Sound during deglaciation is documented by ice dammed lakes at 6.3 kya in Salmon Valley (Jackson et al. 2018) which is consistent with relative sea level reconstructions for the Scott Coast (Denton and Hall 2000 and Hall et al., 2004). Koetillitz Glacier shows a rapid thinning phase between 10.3 kya and 8.3 kya (Anderson et al. 2017) with the onset of thinning at 14 kya (Anderson et al., 2017; Jackson et al., 2018). The total overall ice surface lowering of Koetillitz Glacier (Figure 5.8) is $~ 500 \mathrm{~m}$ (Anderson et al., 2017).

Three marine sediment cores (CH-GC-08, HWD03-1, and ANTA99-CD38) spatially and temporally constrain the overall configuration of grounded ice along the Scott Coast during 
the Holocene. The CH-GC-08 sediment core located $\sim 60 \mathrm{~km}$ east of Ross Island (Figure 5.8) has a facies assemblage of diamict, laminated mud, and diatomatious sediment. This facies succession indicates that open marine conditions existed east of Ross Island by $~ 8.6$ kya (MaKay et al., 2015). The HWD03-1 sediment core collected under the current McMurdo Ice Shelf (Figure 5.8) at Windless Bite, $\sim 10 \mathrm{~km}$ south of Ross Island, has a basal age of $~ 10.1$ kya. The HWD03-1 facies assemblage consists of locally developed unconsolidated muddydiamict, laminated-sands, and interbedded sand and mud facies. Critically this $0.6 \mathrm{~m}$ short core does not contain a compacted diamict, which would indicate grounded ice, however, ANDRILL cores collected nearby do record a compacted daimict at $\sim 1.94 \mathrm{~m}$ depth (Naish et al.,2007). The facies assemblage here suggests long-lived persistent ice-shelf cover from 10.1 kya to the present (MaKay et al., 2007). The sediment core ANTA99-CD38, collected $\sim 200 \mathrm{~km}$ north of Ross Island (Figure 5.8) in the Nordenskjöld Basin (Southern Drygalski Trough) has a facies assemblage dominated by laminated mud and diotomatious sediment with the transition dated to 9.3 kya. This core is interpreted to represent a persistent ice-shelf off the Scott Coast between Mawson and David Glaciers (Finocchiaro et al., 2007) (Figure 5.8).

Collectively the thinning chronology from Koetillitz Glacier and the three marine sediment cores suggest that the large-scale grounded ice retreat in the Ross Sea near the Scott Coast occurred between 10.1 kya and 9.3 kya with open water conditions developing at $~ 8.6$ kya. Open water $60 \mathrm{~km}$ east of Ross Island occurs $\sim 2.1 \mathrm{ky}$ before significant thinning is documented by terrestrial cosmogenic nuclide analysis at Mawson and Mackay glaciers, and $\sim 2.7 \mathrm{ky}$ to $3 \mathrm{ky}$ before dated facies transitions document rapid grounding line retreat toward Mawson and Mackay glaciers (Parker, 2017). The correlation between rapid thinning at Koetillitz at 10.8 kya and the HWD03-1 short core suggests that outlet glaciers in the region did experience thinning but this is not demonstrated at the locations that I sampled. One 
possible reason for this is that thicker, as yet unrecorded ice over topped the sample sites along the Scott Coast, and that this ice was removed in the early Holocene. 


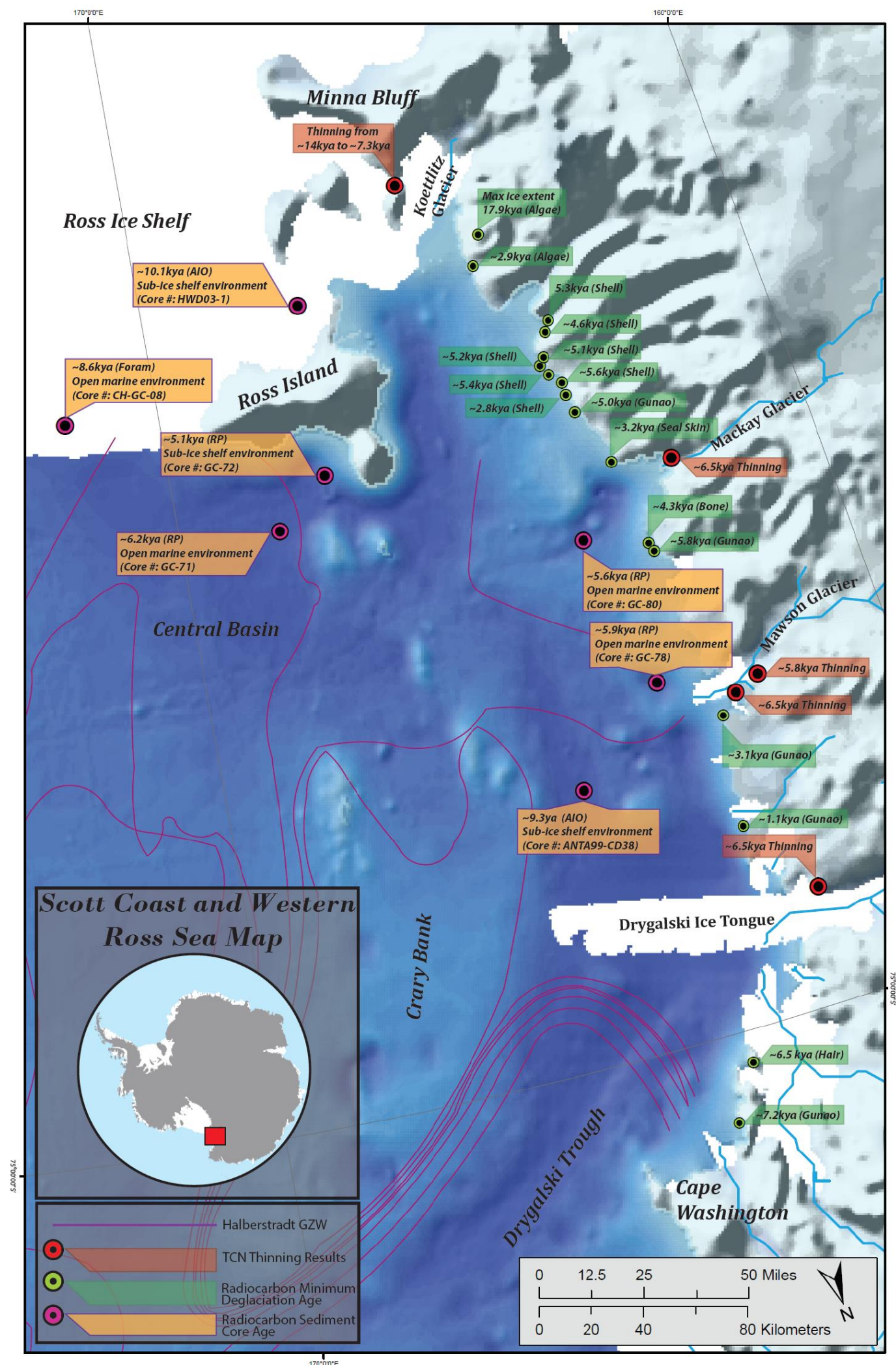

Figure 5.8 Summary map of marine and terrestrial data from the Scott Coast and the McMurdo Sound

This map shows the relationship of marine deglaciation features as well as terrestrial chronologic constraints. The marine sediment cores shown in orange boxes contain the age, material analysed (AIO is Acid Insoluble Organic, RP is ramped Pyrolysis, and Foram is Foraminifera), the environmental interpretation, and the core number. The terrestrial radiocarbon ages are shown in green boxes and include the maximum age from the site and the type of material dated. The terrestrial cosmogenic nuclide studies are shown in red boxes these data only show the onset of rapid thinning. 


\subsubsection{Western Ross Sea bathymetry}

High-resolution bathymetry mapping in the western Ross Sea illuminates and integrates the coupled nature of the Mawson, Mackay, and Koetillitz glacial systems (Halberstadt et al., 2016; Lee et al., 2017 and Greenwood et al., 2018). Mega-scale glacial lineations and grounding zone wedges document the complex nature of the Scott Coast outlet glacier system (Lee et al., 2017). Importantly these landforms document positions of former terminus stability and the orientation of streaming ice flow. Mawson, Mackay, and Koetillitz glaciers flowed east off the coast and coalesced between Franklin and Ross islands where they fed into larger streaming ice moving into the Joides Basin (Lee et al., 2017; Kim et al., 2018) (Figures 1.1 and 5.7).

Several phases of grounding line stability are recorded as grounding zone wedges preserved between the Mawson and Mackay glaciers and Franklin and Ross islands (Halberstadt et al., 2016; Lee et al., 2017; Greenwood et al., 2018). Progressive grounding line retreat along with brief periods of stability have produced a series of grounding zone wedges and recessional moraines which track the overall pattern of retreat in the western Ross Sea (Halberstadt et al., 2016; Lee et al., 2017; Greenwood et al., 2018) (Figure 5.7). While these landforms do record the spatial position of grounded ice off the Scott Coast, many are undated and do not assist in assessing temporal grounding line changes in the western Ross Sea region.

\subsubsection{Regional significance of thinning histories at Mawson and Tucker Glaciers}

Widespread and varied terrestrial and marine datasets from the Scott Coast (Hall et al. 2004; Baroni and Hall, 2004; Jackson et al., 2018; Anderson et al., 2017; Parker, 2017; Jones et al.,2015; MaKay et al., 2015; MaKay et al., 2007, Lee et al., 2017, Greenwood et al., 2018; Finocchiaro et al., 2007; this study) show that grounded ice was maintained in the western 
Ross Sea until $\sim 6.5$ kya which is $\sim 2.5$ ky to $3.5 \mathrm{ky}$ after grounded ice in the Ross Sea region had retreated to Beardmore Glacier and the Siple Coast $\sim 700 \mathrm{~km}$ to $\sim 1000 \mathrm{~km}$ to the south (Spector et al. 2017; Kingslake et al., 2018). Grounded ice in the southwestern Ross Sea must have been maintained by ice flowing from the East Antarctic Ice Sheet into the Ross Embayment. The ice flux through the Transantarctic Mountains along the Scott Coast must have remained high enough to maintain marine based glaciers in the McMurdo Sound region until $\sim 6.5$ kya. Additionally, the Scott Coast system must have been significantly decoupled from the influence of grounded ice in the Ross Sea Region between 10.1 kya and 8.6 kya in accordance with facies transitions from marine sediment cores with a significant episode of collapse documented by outlet glacier thinning. This decoupling would allow for independent interactions with large scale climatic forcings such as an increase in polynya efficiency (Stewart et al., 2019) or a poleward transition in westerly winds which increases circumpolardeep-water (Parker et al., 2017).

These combined data sets provide additional constraints on several hypothesized grounding line retreat scenarios (Conway et al., 1999; Ackert et al., 2008) which aim to explain the evolution of ice retreat in the Ross Embayment. Critically, neither of these hypothesizes consider the Scott Coast Region as an independent area which maintains grounded ice until 6.5 kya. The 'Marine Based' Hypothesis of Halberstadt et al. (2016) does identify a complex network of undated grounding zone wedges throughout the southwestern Ross Sea. These swath-bathymetry data provide a clearer understanding of the nature of grounded ice in the McMurdo Sound region. However, as the grounding zone wedges are undated, the timing and nature of grounded ice retreat in this area is not well constrained but does broadly conform to the marine sediment core ages (Figure 5.8). 


\subsection{Evidence for a Mawson Glacier contribution to Meltwater pulse-1a}

The thinning signal from Mawson Glacier shows rapid thinning from $~ 6.5$ kya to 4.9 kya and gradual thinning from $\sim 4.9$ kya to $1.5 \mathrm{kya}$. The onset of thinning is unconstrained here, therefore, it is unclear if Mawson Glacier was a potential source of significant meltwater during the global deglaciation since the Last Glacial Maximum. The glacial reconstruction from Mackay Glacier indicates that the paleo-ice surface was $\sim 260 \mathrm{~m}$ above the modern ice surface $\sim 20$ kya, however, a sample gap exists between the highest ages (at $\sim 260 \mathrm{~m}$ ) and the onset of rapid thinning $\sim 40$ m lower and $~ 15$ kya later (Jones et al., 2015). Meltwater pulse1a falls within the data gap at Mackay Glacier. Therefore, the ice surface reconstruction from Mackay Glacier does not provide conclusive data as to the role outlet glaciers along the Scott Coast played during Meltwater pulse-1a. The ice surface reconstruction from Koetillitz Glacier, west of Ross Island, shows that thinning starts after $\sim 14$ kya and proceeds until $\sim 8.3$ kya. This ice surface reconstruction is interpreted to show that sea-level rise from Meltwater pulse-1a may have initiated thinning at Koetillitz Glacier, but that Koetillitz Glacier was not a source of meltwater (Anderson et al., 2017). Terrestrial radiocarbon studies in Southern Victoria Land do not show melt-water input from the Scott Coast during Meltwater pulse-1a (between $\sim 14.6$ kya and $\sim 14.3 \mathrm{kya}$ ), but glaciers in the area may respond to rising sea level (Hall and Denton, 2000; Hall et al. 2004, Baroni and Hall, 2004; Hall et al, 2015; Jackson et al., 2018; Perotti et al., 2018). Four thinning histories from outlet glaciers in the southern Transantarctic Mountains are interpreted to show that the Ross Sea Sector neither contributed nor responded in a significant manner to the rapid sea-level rise from Meltwater pulse-1a (Spector et al., 2017). 


\section{TUCKER GLACIER: THINNING SINCE THE LAST GLACIAL MAXIMUM}

\subsection{Introduction}

\section{$\underline{6.1 .1} \underline{\text { Context }}$}

The overall purpose of this chapter is to constrain the glacial history of an outlet glacier that drains the Admiralty Mountains of Northern Victoria Land. Outlet glaciers in much of this region drain large discrete accumulation basins and are entirely independent from the East Antarctic Ice Sheet. Because these glaciers are not connected to the ice sheet, they may behave differently than other large outlet glaciers and ice streams around Antarctica, by responding to drivers such as regional accumulation changes or ocean conditions.

The main trunk of Tucker Glacier is the largest glacier along the Borchgrevink Coast in Northern Victoria Land (Figure 6.1). Tucker Glacier is $\sim 178 \mathrm{~km}$ long and $13.5 \mathrm{~km}$ wide at its terminus with a total catchment size of $\sim 11,480 \mathrm{~km}^{2}$ (Baroni et al., 2005). Tucker Glacier has six distinct large tributary glaciers. Located between the Victory Mountains and the Admiralty Range it occupies a relict fluvial drainage network (Baroni et al., 2005).

Tucker Glacier is near the hypothesised maximum extent of grounded ice in the Western Ross Sea during the Last Glacial Maximum (Shipp et al., 1999; Denton and Hughes 2000). Therefore, the thinning history of Tucker Glacier has the potential to resolve a long-standing debate about the extent of grounded ice in the western Ross Sea. The $150 \mathrm{~km}$ discrepancy in hypothesised grounded ice extent could amount to tens of centimetres of potential sea-level rise. 


\section{$\underline{6.1 .2} \underline{\text { Aim of work }}$}

The aim of this work is to reconstruct the ice surface history of Tucker Glacier since the last glacial maximum.

\subsection{Sample collection and processing}

\section{$\underline{6.2 .1}$ Sample collection}

Glacially-derived material was collected from three locations adjacent to the northern side of Tucker Glacier in November of 2016 (Figure 6.1A). The sites were selected to complement previous work completed on the south margin of Tucker Glacier (Shark Fin site) in 2014 (Balco et al., 2019 and Goehring et al., 2019). The main sampling location for this work is at Heff's Manor, a ridge trending dominantly northwest-southeast near the terminus of Tucker Glacier (Figure 6.1B). It is the eastern most sampled location in the Tucker catchment. The south facing slope is inclined, ice free, and has excellent bedrock exposure (Figure 6.1C). The bedrock is intact but strongly weathered granite of the Admiralty Intrusive Complex (Harrington et al., 1963; Duphorn, 1981). Ten glacial erratics were collected at Heff's Manor and data are presented in tables 6.1 and 6.2. The sample transect had a sampling interval of $\sim 10 \mathrm{~m}$ and was continuous from $\sim 180 \mathrm{~m}$ asl to $\sim 290 \mathrm{~m}$ asl (Figure $6.1 \mathrm{D}$ ). The ice-surfaceelevation measured at this location is approximately $76 \mathrm{~m}$ asl. The position of the transect in the catchment was chosen because it should record dynamic changes in the thickness of Tucker Glacier as a result of grounding line changes. The orientation of the ridge relative to Tucker Glacier, the low slope-angle, type of bedrock, and superb bedrock exposure made it an ideal location to collect samples.

The rocks collected from Heff's Manor are quartz-bearing greywacke of the Roberson Bay Metamorphic suite (Harrington et al., 1963). Samples are brown to grey in colour with no 
defined foliation or lineation present and the average grain size is between $125-63 \mu \mathrm{m}$. The rocks are dominantly comprised of quartz with small percentages of weathered feldspar and other phyllosilicates (see Sample Description in the Method Development chapter). Samples are generally faceted, striated, and bullet shaped with some small spallation pits.

Sample collection was hindered by approximately $0.5 \mathrm{~m}$ of fresh snow at Heff's Manor during the sampling campaign making it difficult to determine the stability of clasts and their proximity to glacially striated and ice moulded bedrock. These factors are useful when collecting samples as they provide context around the deposit and any post-depositional movement of the clast. Snow may also retard the production of ${ }^{10} \mathrm{Be}$ and other terrestrial cosmogenic nuclides. Snow insulates the ground surface from interacting with hadrons produced in the cascade reaction related to cosmic ray/atmosphere interactions (Gosse and Phillips, 2001; Dunai, 2010). If snow cover has been consistently or seasonally present for thousands of years, it will reduce the total number of spallation reactions that occur in the quartz crystal latus, thus, underestimating the sample age (Gosse and Phillips, 2001; Dunai, 2010). Snow cover can be corrected for by using a snow correction formula; however, it requires that the monthly snow depth is known for the location (Whittmann et al., 2006). The mean monthly snow depth is not known for Northern Victoria Land; therefore, an arbitrary correction was not applied to the data. 

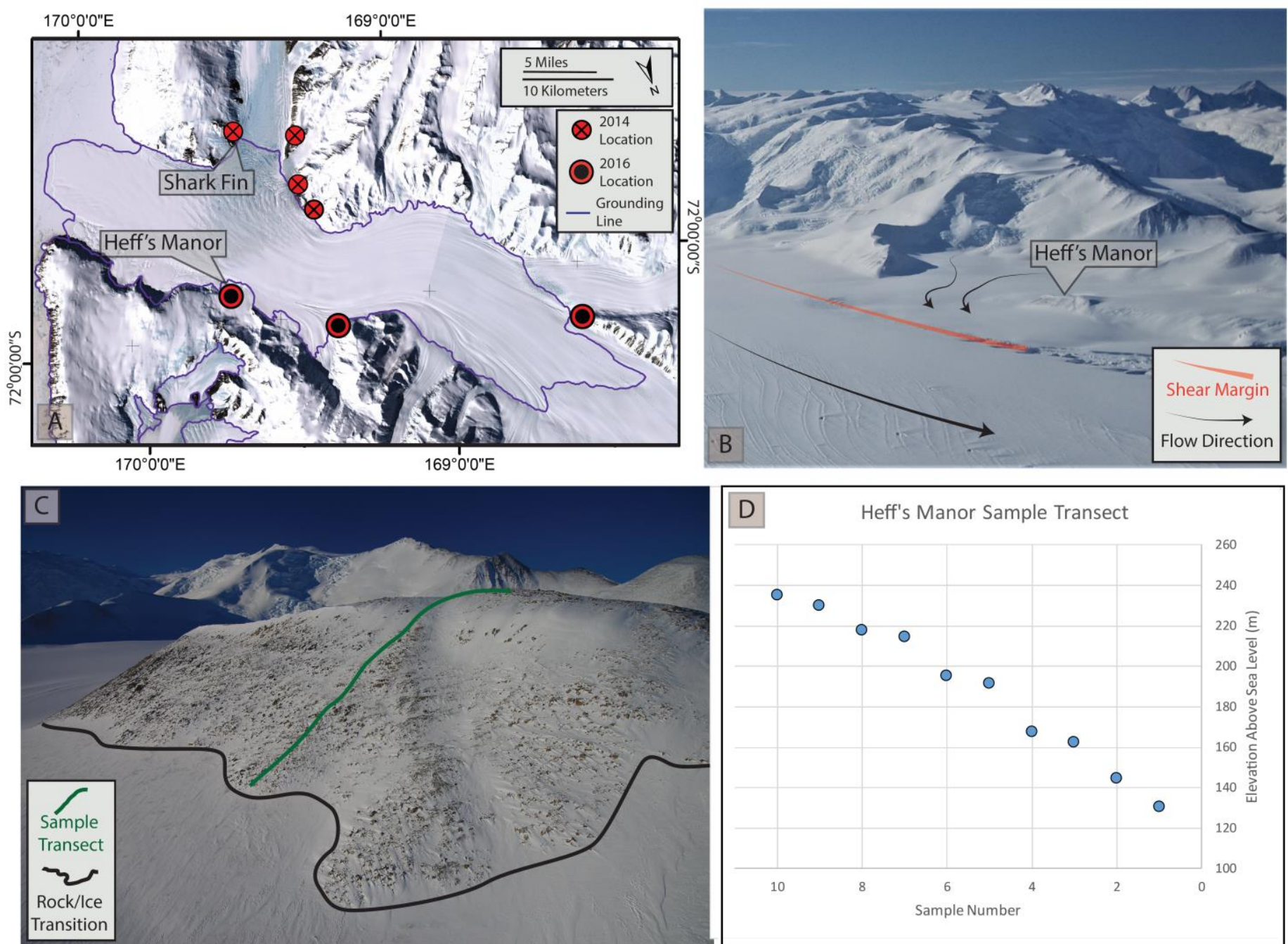

\section{Figure 6.1 Heff's Manor and the Shark Fin sample locations}

Figure 1A: Location map showing the position of Heff's Manor and Shark Fin sample locations as well as all other terrestrial cosmogenic nuclide sample locations in the Tucker Glacier catchment from the 2014 and 2016 seasons. B: Oblique aerial photo showing Heff's Manor and its relation to Tucker Glacier, the northern shear margin and a small local non-dynamic glacier. C: Oblique aerial photo showing the sample transect as well as the local snow cover. D: Graph showing the ten-metre resolution sample elevation profile. 


\section{$\underline{6.2 .2}$ Sample processing}

For the complete quartz purification process and sample processing methods utilized to produce sample ages, please refer to the experimental methods chapter titled "Quantifying the Bucket”.

\section{$\underline{6.2 .3} \underline{\text { Accelerator mass spectrometer analysis }}$}

All samples from Heff's Manor were analysed on an upgraded FN 7 MV tandem accelerator mass spectrometer at PRIME Lab at Purdue University. There were no beam current issues during sample measurement with low uncertainty $(<2.5 \%)$ for all analysed samples.

\subsection{Results}

\section{$\underline{6.3 .1}$ Tucker Glacier chronology}

The 10 exposure ages range from $\sim 15.3 \pm 1.5$ kya to $96.1 \pm 8.2$ kya (Table 6.2 and Figure 6.2). This age-elevation profile (Figure 6.3) has two samples with ages younger than 20,000 years, $\mathrm{HM}-16-04$ is $\sim 15.3 \pm 1.5 \mathrm{kya}$ at $211 \mathrm{~m}$ above the current ice surface and HM-16-10 is $19.3 \pm 1.8 \mathrm{kya}$ at $279 \mathrm{~m}$ above the current ice surface. Eight samples from Heff's Manor have ages greater than 20,000 years and no chronological order was observed (Tables 6.1 and 6.2).

The apparent ages of the eight samples represent a multiple exposure history for each individual clast (Figure 6.3). This complex exposure history does not provide any insight into the thinning history of Tucker Glacier from the Last Glacial Maximum to present. These outliers were removed from the data set. For a complete explanation of factors and processes which complicate an exposure history see the Background section on Cosmogenic Nuclides.

The two post-Last Glacial Maximum samples from Heff's Manor (HM-16-04 and HM-16-10) complement work completed in 2014 at the Shark Fin (Figure 6.4) (Goehring et al., 2019 and 
Balco et al., 2019), on the opposite side of Tucker Glacier. A similar complex exposure history is present for the ${ }^{10} \mathrm{Be}$ data produced on the southern margin of Tucker Glacier. Because 16 of the 18 processed samples showed evidence of inheritance, in-situ ${ }^{14} \mathrm{C}$ was used to resolve a post- Last Glacial Maximum thinning history for the confluence of Tucker and Whitehall glaciers (Goehring et al., 2019). Synthesising the data collected from Heff's Manor and Shark Fin provides additional information on the thinning history of Tucker Glacier (Figure 6.5). 


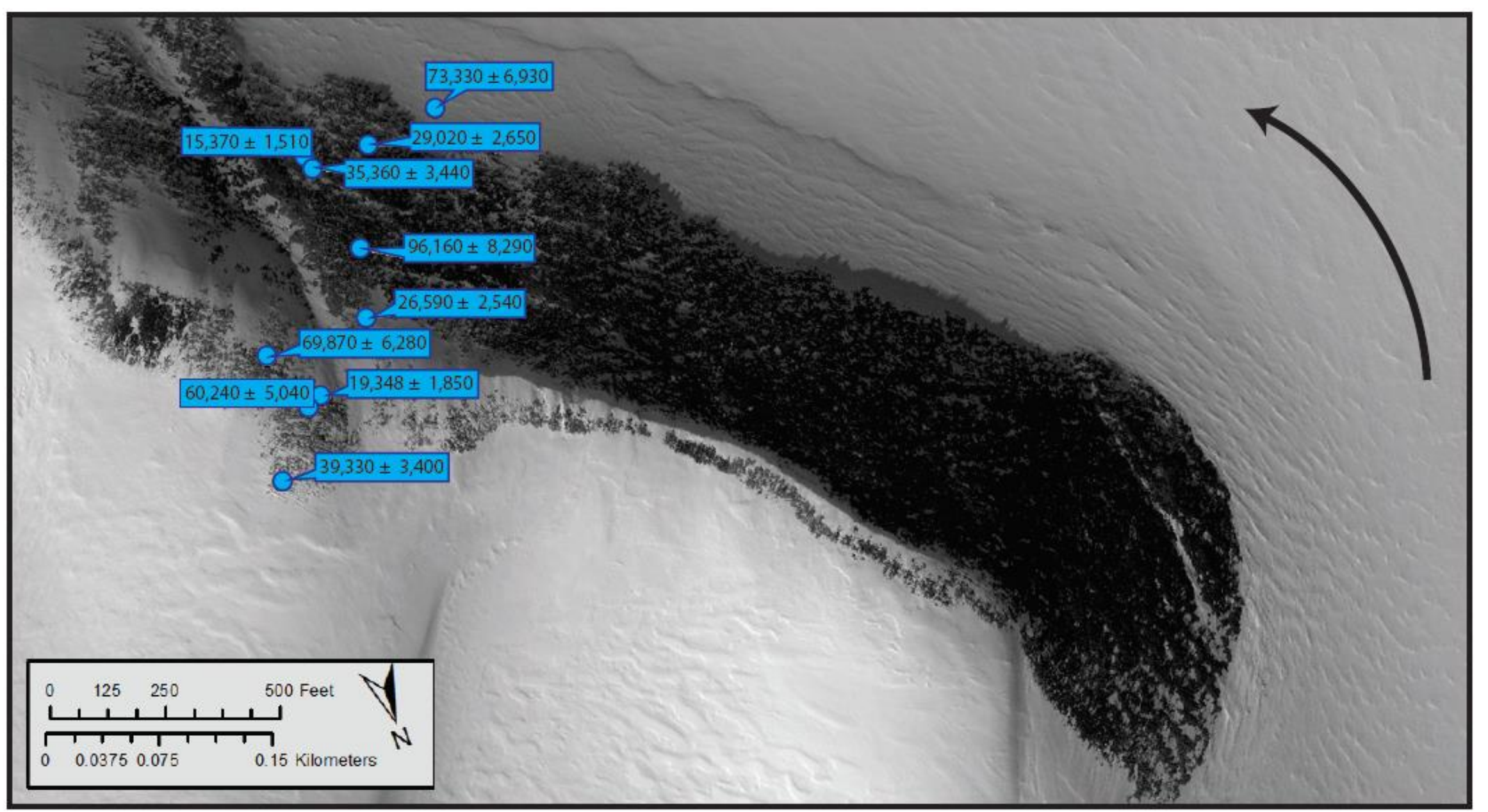

Figure 6.2 Heff's Manor sample locations

This map shows the location and age of samples from Heff's Manor (blue call out boxes and points) along with the general flow orientation of ice around Heff's Manor (black arrow). 


\section{$\underline{6.3 .2}$ Tucker Glacier thinning rate}

The combined Heff's Manor and Shark Fin age elevation profile shows gradual thinning that initiates at $300 \mathrm{~m}$ above the current ice surface $19.3 \pm 1.8$ kya and proceeds unabated to $15 \mathrm{~m}$ elevation at $\sim 5 \pm 0.5$ kya (Figure 6.5 ). The total magnitude of glacier thinning is $\sim 285 \mathrm{~m}$ in $\sim 14 \mathrm{ky}$. The thinning rate is calculated by Monte Carlo least squares linear regression using 10,000 iterations (Figure 6.6). This produces a likely thinning rate for the combined Heff's Manor and Shark Fin dataset of 0.01-0.05 m/yr (95\% confidence). 
Table 6.1 Heff's Manor concentration calculations

This table contains all relevant data for blank correction. ${ }^{10} \mathrm{Be}$ lab blank and total error for each individual The yellow box is the sample blank.

\begin{tabular}{|c|c|c|c|c|c|c|c|c|c|c|c|c|c|}
\hline $\begin{array}{l}\text { Sample } \\
\text { Number }\end{array}$ & $\begin{array}{l}{ }^{10} \mathrm{Be} /{ }^{\prime} \mathrm{Be} \\
\text { Ratio }\end{array}$ & $\begin{array}{l}\text { AMS } \\
\text { Uncertainty }\end{array}$ & $\begin{array}{l}{ }^{9} \mathrm{Be} \\
\text { STD } \\
\text { mass (g) }\end{array}$ & $\begin{array}{l}{ }^{9} \text { Be } \\
\text { STD } \\
(\mathrm{ppm})\end{array}$ & $\begin{array}{l}\text { Standard } \\
\text { Uncertainty } \\
\text { (ppm) }\end{array}$ & $\begin{array}{c}{ }^{9} \mathrm{Be} \\
\text { STD } \\
0\end{array}$ & $\begin{array}{l}{ }^{9} \mathrm{Be} \\
\text { Uncertainty } \\
\text { (g) }\end{array}$ & $\begin{array}{l}\text { Qtz } \\
\text { Mass } \\
\text { (g) }\end{array}$ & $\begin{array}{l}{ }^{10} \text { Be Lab } \\
\text { Blank (at) }\end{array}$ & $\begin{array}{l}\text { Blank } \\
\text { Uncertainty } \\
\text { (at) }\end{array}$ & ${ }^{9} \mathbf{B e}($ at) & ${ }^{10} \mathrm{Be}(\mathrm{at} / \mathrm{g})$ & $\begin{array}{l}{ }^{10} \mathrm{Be} \\
\text { Concentration } \\
\text { Error (at/g) }\end{array}$ \\
\hline HM-02 & $1.02 \mathrm{E}-12$ & $2.64 \mathrm{E}-14$ & 0.8794 & 305 & 3 & $2.682 \mathrm{E}-04$ & $2.64 \mathrm{E}-06$ & 40.4406 & $7.16 \mathrm{E}+04$ & $1.10 \mathrm{E}+04$ & $1.79 \mathrm{E}+19$ & $4.51 \mathrm{E}+05$ & $1.25 \mathrm{E}+04$ \\
\hline HM-03 & $5.07 \mathrm{E}-13$ & $1.22 \mathrm{E}-14$ & 0.8798 & 305 & 3 & 2.683E-04 & 2.64E-06 & 51.6592 & $7.16 \mathrm{E}+04$ & $1.10 \mathrm{E}+04$ & $1.79 \mathrm{E}+19$ & $1.75 \mathrm{E}+05$ & $4.57 \mathrm{E}+03$ \\
\hline HM-04 & $2.17 \mathrm{E}-13$ & $6.28 \mathrm{E}-15$ & 0.8781 & 305 & 3 & $2.678 \mathrm{E}-04$ & 2.63E-06 & 38.5118 & $7.16 \mathrm{E}+04$ & $1.10 \mathrm{E}+04$ & $1.79 \mathrm{E}+19$ & $9.90 \mathrm{E}+04$ & $3.10 \mathrm{E}+03$ \\
\hline HM-05 & $5.72 \mathrm{E}-13$ & 1.62E-14 & 0.8804 & 305 & 3 & $2.685 \mathrm{E}-04$ & $2.64 \mathrm{E}-06$ & 48.3753 & $7.16 \mathrm{E}+04$ & $1.10 \mathrm{E}+04$ & $1.79 \mathrm{E}+19$ & $2.11 \mathrm{E}+05$ & $6.37 \mathrm{E}+03$ \\
\hline HM-06 & $1.11 \mathrm{E}-12$ & $2.11 \mathrm{E}-14$ & 0.8786 & 305 & 3 & $2.680 \mathrm{E}-04$ & $2.64 \mathrm{E}-06$ & 32.7619 & $7.16 \mathrm{E}+04$ & $1.10 \mathrm{E}+04$ & $1.79 \mathrm{E}+19$ & $6.07 \mathrm{E}+05$ & $1.30 \mathrm{E}+04$ \\
\hline HM-07 & $4.75 \mathrm{E}-13$ & $1.29 \mathrm{E}-14$ & 0.88 & 305 & 3 & $2.684 \mathrm{E}-04$ & 2.64E-06 & 46.6764 & $7.16 \mathrm{E}+04$ & $1.10 \mathrm{E}+04$ & $1.79 \mathrm{E}+19$ & $1.81 \mathrm{E}+05$ & $5.28 \mathrm{E}+03$ \\
\hline HM-08 & $1.54 \mathrm{E}-12$ & 3.44E-14 & 0.8797 & 305 & 3 & 2.683E-04 & 2.64E-06 & 58.4364 & $7.16 \mathrm{E}+04$ & $1.10 \mathrm{E}+04$ & $1.79 \mathrm{E}+19$ & $4.71 \mathrm{E}+05$ & $1.15 \mathrm{E}+04$ \\
\hline HM-09 & $1.48 \mathrm{E}-13$ & $3.95 \mathrm{E}-15$ & 0.8792 & 305 & 3 & 2.682E-04 & $2.64 \mathrm{E}-06$ & 18.9811 & $7.16 \mathrm{E}+04$ & $1.10 \mathrm{E}+04$ & $1.79 \mathrm{E}+19$ & $1.36 \mathrm{E}+05$ & $4.01 \mathrm{E}+03$ \\
\hline HM-10 & $6.51 \mathrm{E}-13$ & $1.14 \mathrm{E}-14$ & 0.8788 & 305 & 3 & $2.680 \mathrm{E}-04$ & $2.64 \mathrm{E}-06$ & 28.5816 & $7.16 \mathrm{E}+04$ & $1.10 \mathrm{E}+04$ & $1.79 \mathrm{E}+19$ & $4.06 \mathrm{E}+05$ & $8.18 \mathrm{E}+03$ \\
\hline HM-11 & 4.63E-13 & $9.23 \mathrm{E}-15$ & 0.8794 & 305 & 3 & $2.682 \mathrm{E}-04$ & $2.64 \mathrm{E}-06$ & 30.5831 & $7.16 \mathrm{E}+04$ & $1.10 \mathrm{E}+04$ & $1.79 \mathrm{E}+19$ & $2.69 \mathrm{E}+05$ & $6.04 \mathrm{E}+03$ \\
\hline BLANK & 3.99E-15 & $6.11 \mathrm{E}-16$ & 0.8799 & 305 & 3 & 2.684E-04 & 2.64E-06 & 0 & $7.16 \mathrm{E}+04$ & $1.10 \mathrm{E}+04$ & $1.79 \mathrm{E}+19$ & & \\
\hline
\end{tabular}


Table 6.2 Heff's Manor age calculation and apparent exposure age

Calculated Sample ages reported in LDSn. The Two usable samples, HM-16-04 and HM-16-10, are shown in blue below. The eight other out of chronologic order samples are shown in red.

\begin{tabular}{|c|c|c|c|c|c|c|c|c|c|c|c|c|c|c|}
\hline $\begin{array}{l}\text { Sample } \\
\text { Number }\end{array}$ & $\begin{array}{l}\text { Latitude } \\
\text { (DD) }\end{array}$ & $\begin{array}{l}\text { Longitude } \\
\text { (DD) }\end{array}$ & $\begin{array}{l}\text { Elevation } \\
\text { (m) }\end{array}$ & ATM & $\begin{array}{l}\text { Thick- } \\
\text { ness } \\
\text { (cm) }\end{array}$ & $\begin{array}{l}P \\
\mathrm{~g} / \mathrm{cm}^{3}\end{array}$ & $\begin{array}{l}\text { Shield- } \\
\text { ing }\end{array}$ & $\begin{array}{l}\text { Erosion } \\
\text { Rate } \\
(\mathbf{m} / \mathbf{M y r})\end{array}$ & $\begin{array}{l}{ }^{10} \mathrm{Be} \\
\text { Concentration } \\
(\text { at/g) }\end{array}$ & $\begin{array}{l}{ }^{10} \mathrm{Be} \\
\text { Uncertainty } \\
(\text { at } / g)\end{array}$ & $\begin{array}{l}{ }^{10} \mathrm{Be} \\
\text { Standard }\end{array}$ & $\begin{array}{l}\text { Age } \\
(\mathbf{y r})\end{array}$ & $\begin{array}{l}\text { Internal } \\
\text { Uncertainty } \\
(\mathbf{y r})\end{array}$ & $\begin{array}{l}\text { External } \\
\text { Uncertainty } \\
\text { (yr) }\end{array}$ \\
\hline HM-02 & -72.53462409 & 169.66223947 & 180.004 & ant & 2.70 & 2.7 & 0.9706 & 0 & $4.51 \mathrm{E}+05$ & $1.25 \mathrm{E}+04$ & 07KNSTD & 73332 & 2070 & 4869 \\
\hline HM-03 & -72.53450193 & 169.66336978 & 196.69 & ant & 7.90 & 2.7 & 0.9645 & 0 & $1.75 \mathrm{E}+05$ & $4.57 \mathrm{E}+03$ & 07KNSTD & 29026 & 764 & 1886 \\
\hline HM-04 & -72.53446441 & 169.66453342 & 211.405 & ant & 3.50 & 2.7 & 0.9742 & 0 & $9.90 \mathrm{E}+04$ & $3.10 \mathrm{E}+03$ & 07KNSTD & 15373 & 483 & 1031 \\
\hline HM-05 & -72.53439885 & 169.66438947 & 213.828 & ant & 7.30 & 2.7 & 0.9351 & 0 & $2.11 \mathrm{E}+05$ & $6.37 \mathrm{E}+03$ & 07KNSTD & 35369 & 1077 & 2365 \\
\hline HM-06 & -72.53395698 & 169.66380140 & 233.524 & ant & 5.40 & 2.7 & 0.9715 & 0 & $6.07 \mathrm{E}+05$ & $1.30 \mathrm{E}+04$ & 07KNSTD & 96167 & 2110 & 6184 \\
\hline HM-07 & -72.53360869 & 169.66393906 & 254.37 & ant & 2.50 & 2.7 & 0.9831 & 0 & $1.81 \mathrm{E}+05$ & $5.28 \mathrm{E}+03$ & 07KNSTD & 26597 & 781 & 1762 \\
\hline HM-08 & -72.53350052 & 169.66569829 & 274.439 & ant & 6.20 & 2.7 & 0.9966 & 0 & $4.71 \mathrm{E}+05$ & $1.15 \mathrm{E}+04$ & 07KNSTD & 69873 & 1736 & 4540 \\
\hline HM-09 & -72.53323573 & 169.66494729 & 278.188 & ant & 3.20 & 2.7 & 0.9951 & 0 & $1.36 \mathrm{E}+05$ & $4.01 \mathrm{E}+03$ & 07KNSTD & 19348 & 573 & 1282 \\
\hline HM-10 & -72.53320344 & 169.66518518 & 279.672 & ant & 6.90 & 2.7 & 0.9943 & 0 & $4.06 \mathrm{E}+05$ & $8.18 \mathrm{E}+03$ & 07KNSTD & 60247 & 1232 & 3813 \\
\hline HM-11 & -72.53282981 & 169.66588766 & 286.603 & ant & 7.20 & 2.7 & 0.999 & 0 & $2.69 \mathrm{E}+05$ & $6.04 \mathrm{E}+03$ & 07KNSTD & 39331 & 892 & 2507 \\
\hline
\end{tabular}




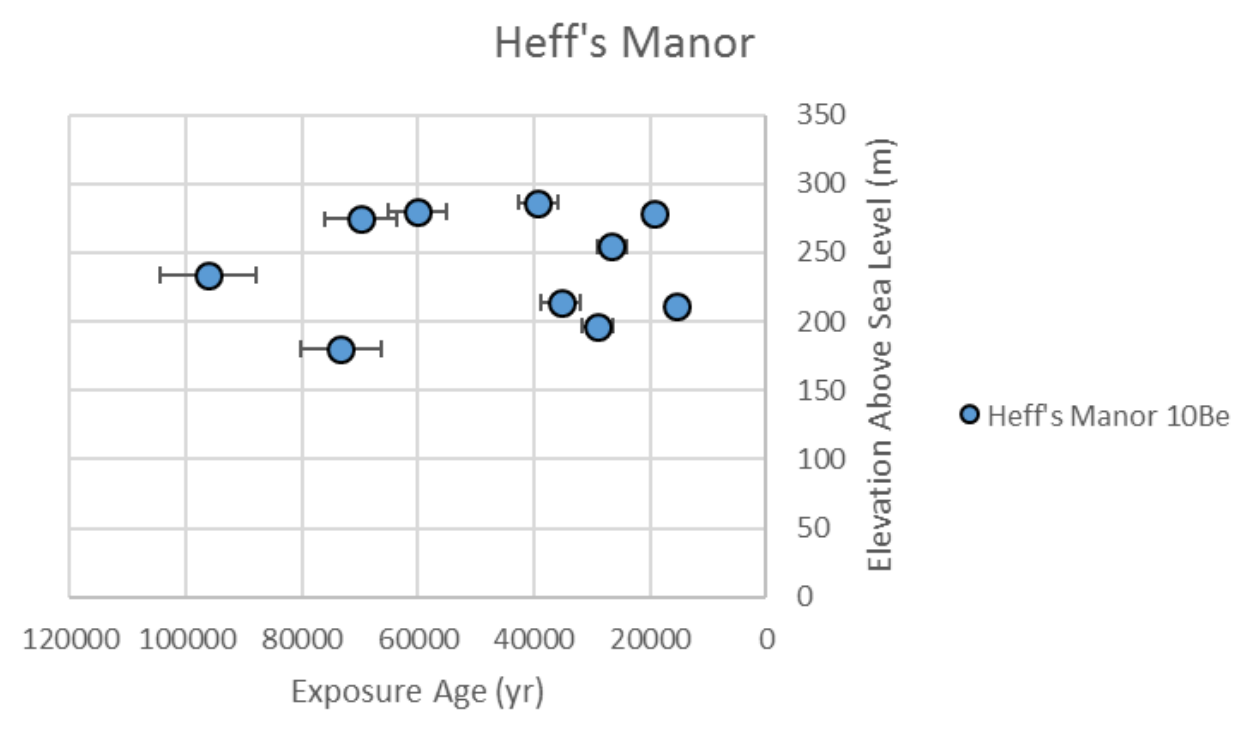

\section{Figure 6.3 Heff's Manor chronology}

Plotted are all samples collected from Heff's Manor. The two youngest samples represent the post- Last Glacial Maximum thinning history of Tucker Glacier. The eight other points imply a complex multiple exposure history unique to each individual sample. The nature of clast recycling and multiple exposure means that the true exposure pathway is obscured. However, this type of exposure pattern does provide limited insight to how sediment moves through the large complex glacial tributaries.

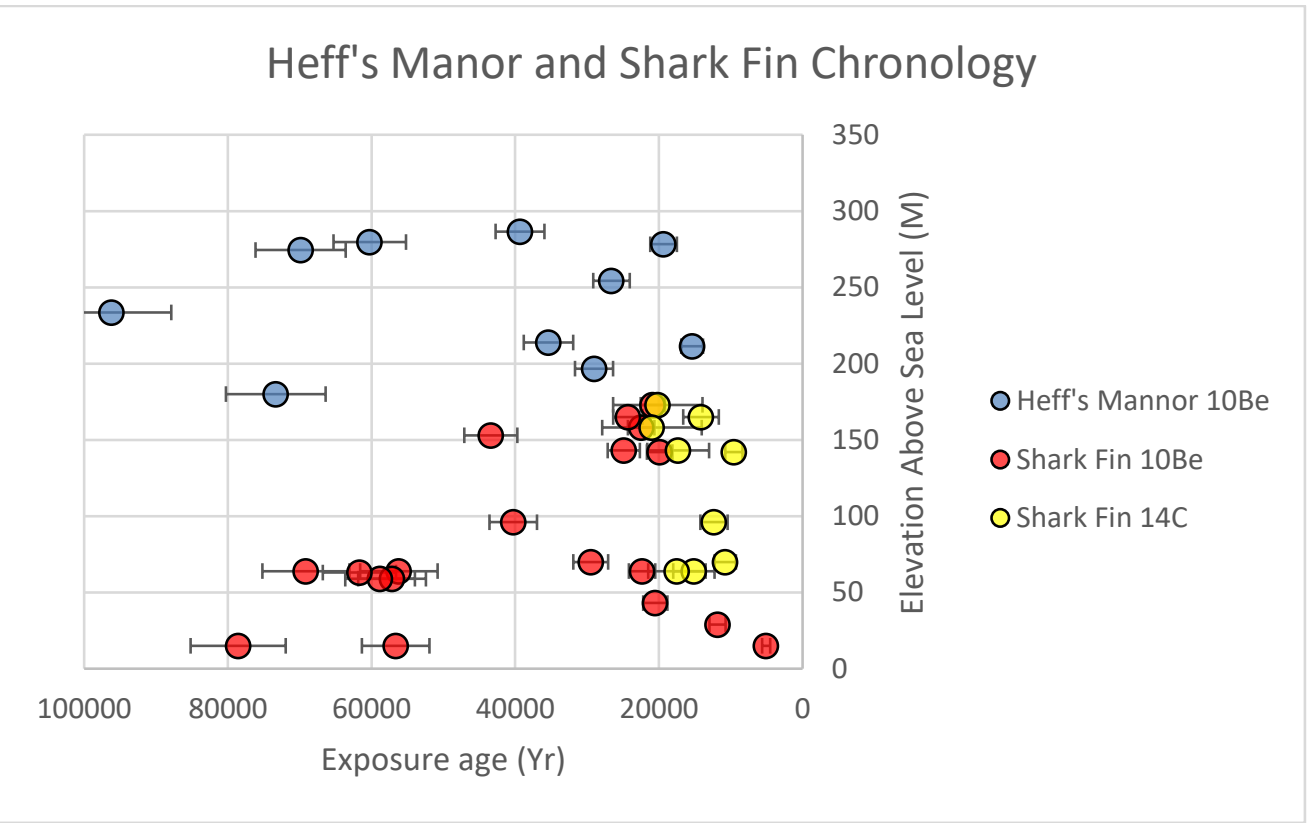

\section{Figure 6.4 Heff's Manor and Shark Fin chronology}

This age elevation plot is a compilation of the Heff's Manor and Shark Fin chronologies. The data are broken down by nuclide and location for ease of interpretation with Heff's Manor ${ }^{10}$ Be shown in blue, Shark Fin ${ }^{10}$ Be shown in red, and Shark Fin ${ }^{14} \mathrm{C}$ shown in yellow. The majority of the samples at Heff's Manor and Shark Fin likely record multiple exposure histories. 


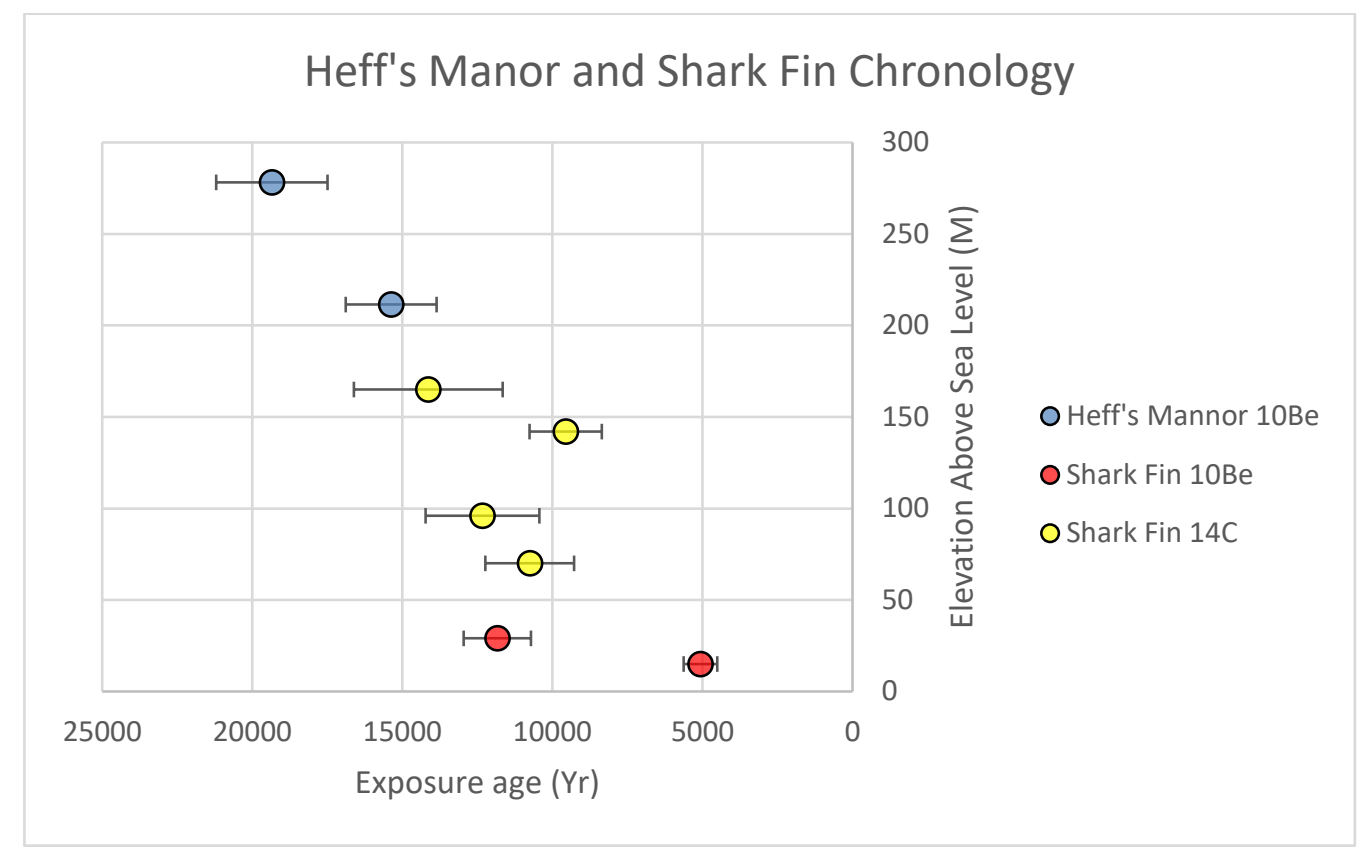

Figure 6.5 Simplified Heff's Manor and Shark Fin chronology

All samples showing a multiple exposure history are removed from this plot to clarify the post- Last Glacial Maximum thinning history of Tucker Glacier. The sample transect starts at $\sim 280 \mathrm{~m}$ asl and drops to $\sim 15 \mathrm{~m}$ asl.

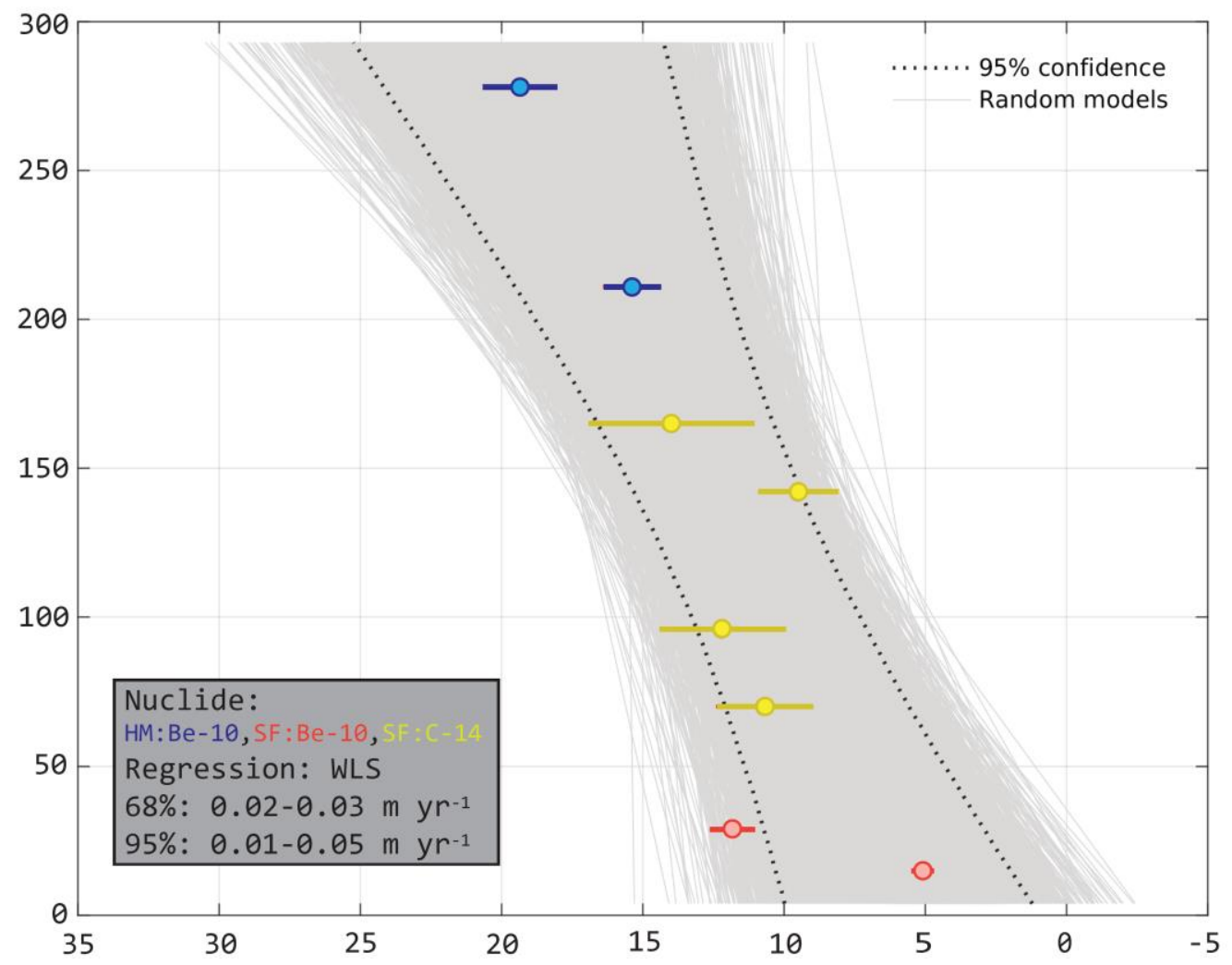

Figure 6.6 Monte Carlo least squares linear regression for Heff's Manor and the Shark Fin

The thinning rate is calculated by applying a 10,000 iteration Monte Carlo least squares linear regression analysis to the thinning profile in figure 6.4. The colour convention for this plot is the same as above: ${ }^{10} \mathrm{Be}$ from Heff's Manor is blue, ${ }^{10} \mathrm{Be}$ from the Shark Fin is red, and ${ }^{14} \mathrm{C}$ from the Shark Fin is yellow. Tucker Glacier's thinning rate is $0.01-0.05 \mathrm{~m} / \mathrm{yr}$ (95\% confidence). The thinning rate was calculated using iceTEA (Jones et al., 2019). 


\section{$\underline{6.3 .3}$ Sample outliers}

Due to the large range of exposure ages which were out of age-elevation chronologic order thirty samples were removed from the integrated Heff's Manor and Shark Fin dataset in order to clarify the post- Last Glacial Maximum thinning history. The sample outliers present an opportunity for future work and this idea is further explored in the Future Work section. Sample outliers are highlighted in red in table 6.2 and have been removed from figure 6.5. One or many of the complicating factors discussed below may explain the antiquity of many samples from both data sets. The ground distribution is shown in figure 6.2 to illustrate the complex environment surrounding Heff's Manor.

The large number of samples with a multiple exposure history may be the result of complex sediment input by colluvial processes, multiple generations of non-erosive cold-based ice cover, initial exposure in lateral or medial moraine high up in the Tucker Glacier catchment, or multiple clast entrainment/deposition cycles. Each of these potential scenarios would produce a clast with a complex exposure history.

\subsection{Discussion of Tucker Glacier's thinning history}

The age-elevation transect from Heff's Manor is a useful extension to the work completed at the Shark Fin, because both locations occupy similar geographic and glaciologic positions in the catchment of Tucker Glacier (Figure 6.1A). By combining the ice-surface elevation history for both locations, a larger elevation range is covered which results in a longer temporal record. This extended ice-surface-elevation profile tracks the thinning history of Tucker Glacier for the last $\sim 19$ kya (Figure 6.5 ). The data sets are integrated according to the sample elevation above the current ice shelf. The additional $100 \mathrm{~m}$ of elevation provided by Heff's Manor mirrors work completed at Crater Cirque on the southern margin of Tucker Glacier (Balco et 
al., 2019 and Goehring et al., 2019). The data from both Heff's Manor and Crater Cirque show that Tucker Glacier was at least $300 \mathrm{~m}$ thicker during the Last Glacial Maximum (Balco et al., 2019 and Goehring et al., 2019). The gradual style and moderate magnitude of thinning recorded at Tucker Glacier (Figure 6.6) are strikingly different to that reconstructed for other outlet glaciers along the Scott Coast of Southern Victoria Land (Chapter 5).

\subsubsection{Glacial history of the Northwestern Ross Sea}

A key issue raised in Balco et al. (2019) is whether Tucker Glacier was confluent with grounded ice in the Ross Embayment during the Last Glacial Maximum. The gradual style, low rate, timing and duration of thinning at Tucker Glacier, suggest that this outlet glacier may not have been confluent with grounded ice in the Ross Embayment (This Study). This is contrary to the discussion of Goehring and others (2019), who argue that Tucker Glacier was confluent with grounded ice in the Ross Embayment. Their argument centres on high resolution bathymetric data collected in the Ross Sea (Halberstadt et al., 2016), however, the bathymetric data set offshore of Tucker Glacier only contains iceberg scour marks and does not retain a grounded ice record (Figure 6.7). While it is clear that Tucker Glacier thickened and likely extended into the Ross Sea, further work is needed to constrain the grounding line position during previous glacial advances. The closest documented grounding zone wedges and megascale glacial lineations suggest a stable grounding line position for ice in the Ross Embayment approximately $40 \mathrm{~km}$ southeast of Tucker Glacier near Coulman Island (Shipp et al., 1999). 


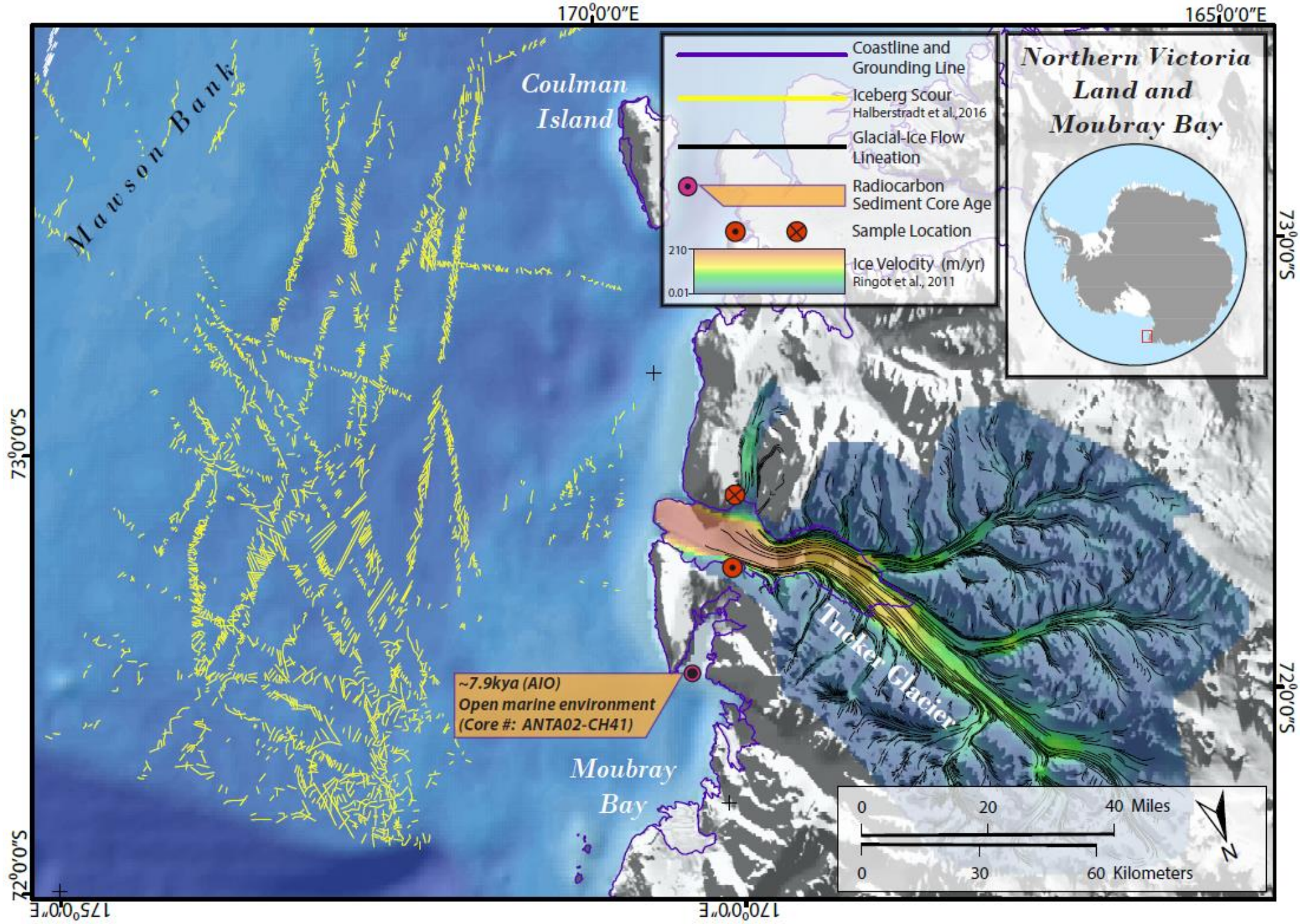

Figure 6.7 Bathymetric record offshore Tucker Glacier

This figure shows the Halberstadt et al., 2016 data set in context to Tucker Glacier. The yellow lines offshore of Tucker Glacier are scour marks produced by icebergs grounding on the seafloor. These features are semi-linear and curvilinear trenches produced when the keel of an iceberg hits the sea floor and disturbs the sediment there. The sheer number of iceberg scours is a testament to the amount of ice calved in the Ross Embayment since the Last Glacial Maximum. 
A recent, regional scale ice sheet modelling study was carried out in the Ross Sea region using the Parallel Ice Sheet Model (PISM) (Lowry et al., 2019). The simulation was initialized with grounded ice extending out to the continental shelf as described in Denton and Hughes (2000). The model output for Tucker Glacier does not accurately replicate the style, magnitude, rate, timing, or duration of thinning (Figure 8.8) represented by the sample ages presented by Balco and others (2019) and Goehring and others (2019). Additionally, the modelling effort is not faithful to the findings presented in Balco and others (2019) or Goehring and others (2019), which states that samples over 390 meters in elevation were not covered by ice. The 17,700 \pm 5,400 kya in-situ ${ }^{14} \mathrm{C}$ sample age shown in the data-model intercomparison (Figure 6.8) at $517 \mathrm{~m}$ (14-TGU-040-SKB) is flanked by two saturated ${ }^{14} \mathrm{C}$ samples at $390 \mathrm{~m}$ (14-TGU-041$\mathrm{SKB})$ and $558 \mathrm{~m}$ (14-TGU-039-SKB) not shown on the data-model intercomparison. The saturated samples indicate continuous exposure for the last 25,000 years therefore, the $\sim 17$ kya age sample was likely moved by periglacial or colluvial processes (Balco et al., 2019; Goehring et al., 2019). The work by Lowry and others (2019) did not consider the data presented by both Balco and others (2019) and Goehring and others (2019), and their ice sheet modelling overestimates the ice thickness and does not replicate the style, magnitude, timing, or rate of thinning at Tucker Glacier. The modelling of Lowry and others (2019) does suggests that if Tucker Glacier was confluent with grounded ice in the Ross Embayment then the ice surface at Tucker Glacier would thicken to $\sim 600 \mathrm{~m}$ above sea level and rapidly retreat into an overdeepened basin once the grounded ice was removed (Figure 6.8) (Lowry et al., 2019). 


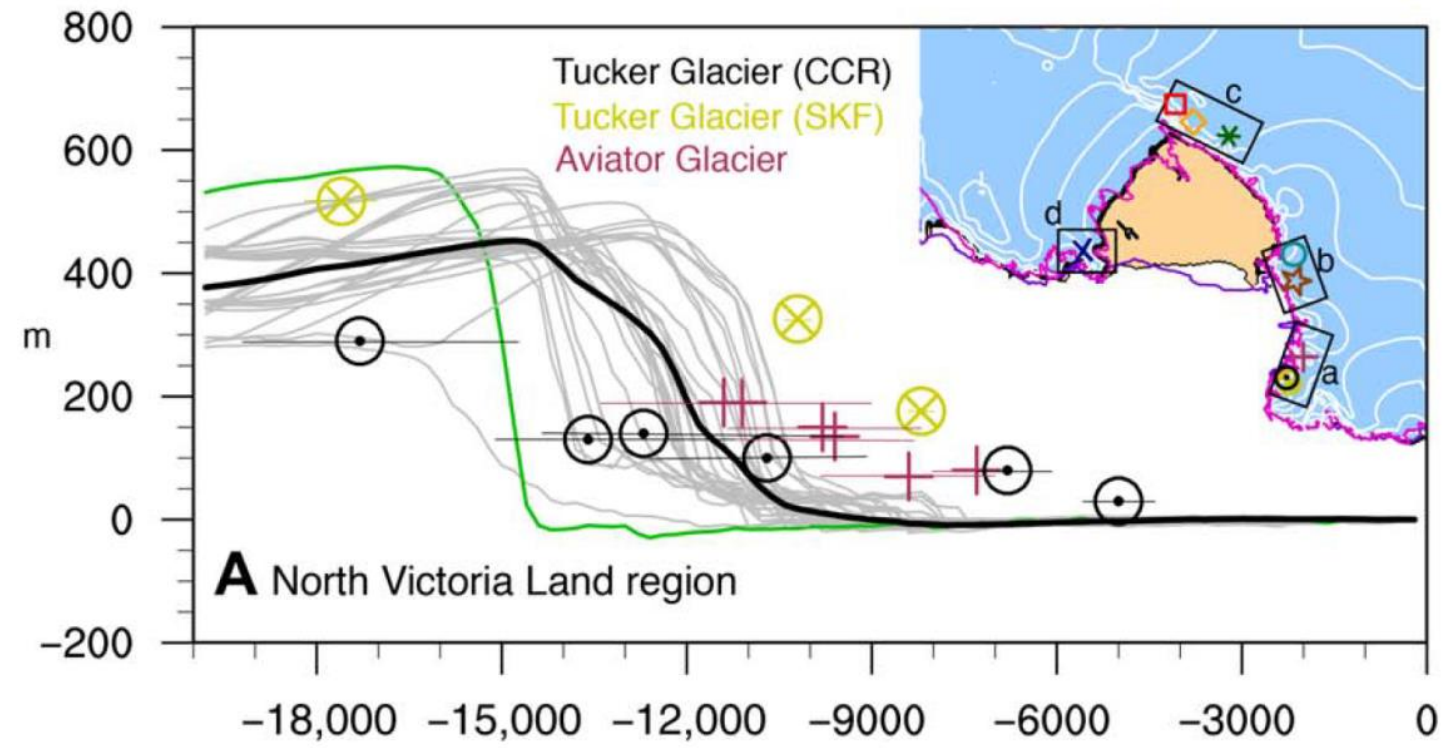

\section{Figure 6.8 Tucker Glacier model}

This data model comparison from Lowry and others (2019) shows individual model runs in grey, the ensemble average is in black, and the green line indicates an early retreat/Holocene re-advance scenario and the exposure ages are shown by the black (Tucker Glacier), yellow (Tucker Glacier), and fuchsia (Aviator Glacier) points of the figure. The modelling was constrained using terrestrial cosmogenically derived ages. The style of thinning is quite dissimilar between the model simulations and the terrestrial cosmogenic ages. Additionally, the timing of ensemble average thinning is years too early.

Together, the relatively slow and continuous nature of ice sheet thinning at Tucker Glacier along with the model-data mismatch implies that the maximum grounded ice extent during the Last Glacial Maximum was south of Tucker Glacier and likely at or near grounding zone wedges identified by Shipp and others (1999). This suggests that Tucker Glacier may have been forced by climatic variables independent of the buttressing effect of grounded ice in the Ross Embayment. However, it is still possible that Tucker Glacier was buttressed by a local ice shelf prior to thinning and subsequent thinning may have been the result of partial or total loss of back stress from such an ice shelf.

Gradual thinning is hypothesized in both Balco and others (2019) and Goehring and others (2019) to reflect grounding line retreat related from the onset of deglaciation until $\sim 5 \mathrm{kya}$ when the glacier appears to have reached a geometry similar to its current configuration (Balco et al., 2019; Goehring et al., 2019, This Study). Balco and others (2019) and Goehring and others 
(2019) suggest that rising sea level drove the retreat. However, other ocean forcing, atmospheric forcing, and isostatic effects would have likely affected the grounding line position of Tucker Glacier and it is not yet possible to say which (if any) of these processes was the dominant driver of gradual glacial thinning documented in this study.

As with many geological and glaciological questions, there are many non-unique solutions which can satisfactorily explain the observations. Therefore, a systematic glacier modelling study of Tucker Glacier should be conducted. Balco and others (2019) attempted to model Tucker Glacier using a flow line model. This simulation ended unsuccessfully due to a lack of available data (Balco et al., 2019). Given that Tucker Glacier is an $\sim 12,000 \mathrm{~km}^{2}$ alpine glacier with six distinct accumulation zones it is unsurprising that a single flow line model could not adequately capture the complexity of this glacier. A more suitable model to use would be a finite-element model, such as Ùa (Gudmundsson et al., 2012).

\subsection{Evidence of a Tucker Glacier contribution to Meltwater pulse-1a}

The results from this thesis demonstrate that Tucker Glacier did not experience rapid thinning at any time during the last deglaciation between $\sim 14,650$ and $\sim 14,310$ years ago during Meltwater pulse 1a. Therefore, Tucker Glacier could not have contributed to rapid sea-level rise during this time. 


\section{EROSION RATES IN VICTORIA LAND: LANDSCAPE EVOLUTION}

\subsection{Introduction}

\section{$\underline{7.1 .1}$ Context}

The hyperarid climate of Antarctic produces some of the lowest bedrock erosion rates in the world (Portenga et al., 2011; Marrero et al., 2018). However, approximately $40 \%$ of the current bedrock erosion rate studies in Antarctica are concentrated in the McMurdo Dry Valleys (e.g. Brook et al., 1993, and Middleton et al., 2012) while 55\% are from high elevation inland sites (e.g. Nishiizumi et al., 1991 and Di Nicola et al., 2012). These studies have demonstrated consistently low bedrock erosion rates, but the spatially confined and environmentally restricted reality of where these data were collected cannot be overlooked. The low erosion rates found in the McMurdo Dry Valleys and at high elevation near the ice sheet may not be representative of coastal regions more generally. The few existing coastal erosion rate studies suggest rock surface lowering rates between $15 \mathrm{~m} / \mathrm{Myr}$ and $22 \mathrm{~m} / \mathrm{Myr}$ (Spate et al., 1995). There are other qualitative studies which provide indirect constraints on the overall weatherability of specific rock types (Gore et al., 1996; Augustinus, 2002; White et al., 2009).

The relationship between temperature/humidity and erosion rate in Antarctica is poorly understood. However, evidence suggests that the right temperature and moisture scenarios may be a significant factor in weathering rock in addition to the porosity, grain size, and microcracking of the rock (Elliott et al., 2008). The coastal areas in the Cape Adare region of Northern Victoria Land have relatively warmer more humid climate as compared to areas at high elevation near the ice sheet. Therefore, the higher temperatures and higher humidity of coastal regions in northern Victoria Land may produce higher bedrock erosion rates. 


\section{$\underline{7.1 .2}$ Aim of work}

The aim of this work is to assess how latitude, altitude, and continentality affect bedrock erosion rates in Northern Victoria Land, Antarctica.

\subsection{Locations}

Bedrock was sampled at several locations along the Victoria Land Coast with different rock types. These sample sites are at lower elevations, relatively close to the coast (Figure 7.1), and outside of the main katabatic corridors of the Reeves and Rennick glaciers. In this section I use phrase distance from the coast to represent distance from seasonally open water in the Edisto Inlet at Cape Christie. 


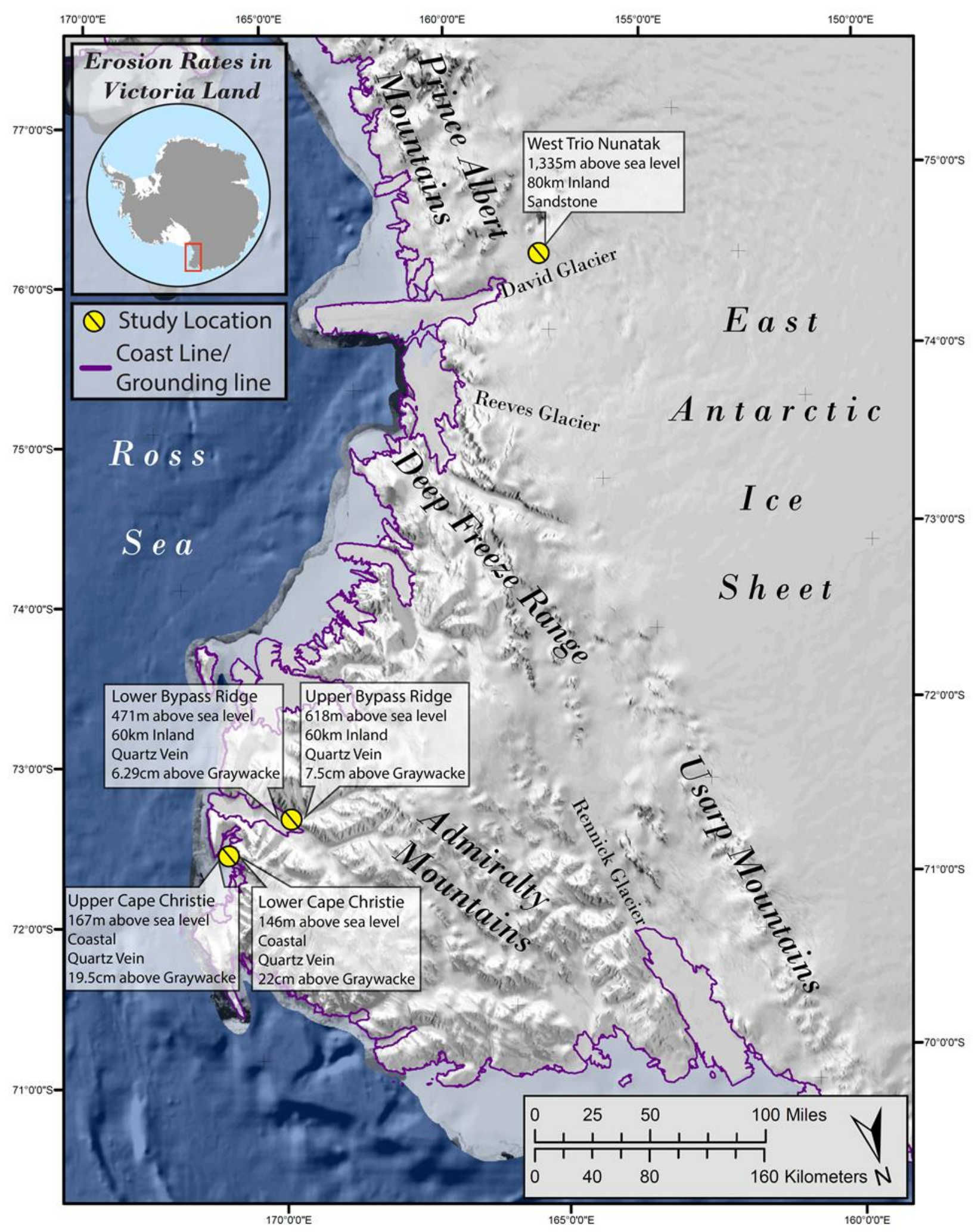

Figure 7.1 Sampling locations in Victoria Land: Antarctica

This figure shows the location of bedrock samples collected for erosion rate analysis in Victoria Land. Each location shows the sample elevation distance from the coast, rock type, and height above the ground surface for the differential erosion rate samples. 


\section{$\underline{7.2 .1}$ West Trio Nunatak}

\subsubsection{Sample collection}

A bedrock sample was collected from West Trio Nunatak in December 2017 as part of a sampling campaign to David Glacier. The Trio Nunataks are a group of three nunataks located at $75^{\circ} 30^{\prime}$ S $159^{\circ} 42^{\prime}$ E near the grounding line of David Glacier in the Prince Albert Mountains, $\sim 80 \mathrm{~km}$ inland from the Scott Coast (Figure 7.1). West Trio Nunatak reaches an elevation of $\sim 1320 \mathrm{~m}$ and is located along the southern margin of David Glacier. The east facing slope is gentle to steeply inclined with intermittent exposure of bedrock and extensive sediment developed by periglacial processes. The bedrock is dominantly composed of Ferrar Dolorite with local slivers of Beacon Sandstone occurring as large, lightly metamorphosed sandstone blocks. The site was selected because it is at a relatively high altitude near the East Antarctic Ice Sheet north of the McMurdo Dry Valleys and helps to fill a data gap in the published literature. The sampling site was located $\sim 60 \mathrm{~m}$ above landforms associated with a glacial trimline. The joint spacing is $\sim 20 \mathrm{~cm}$ with an $\sim 1 \mathrm{~cm}$ dry rough gap, and a ubiquitous periglacial sediment veneer nearby. Landforms present lower on West Trio Nunatak and the lack of glacial erratics near the sample location indicate that this area was above David Glacier's trimline during the last glacial maximum. Bedrock was collected here to constrain the preLast Glacial Maximum glacial history and erosion rate. 


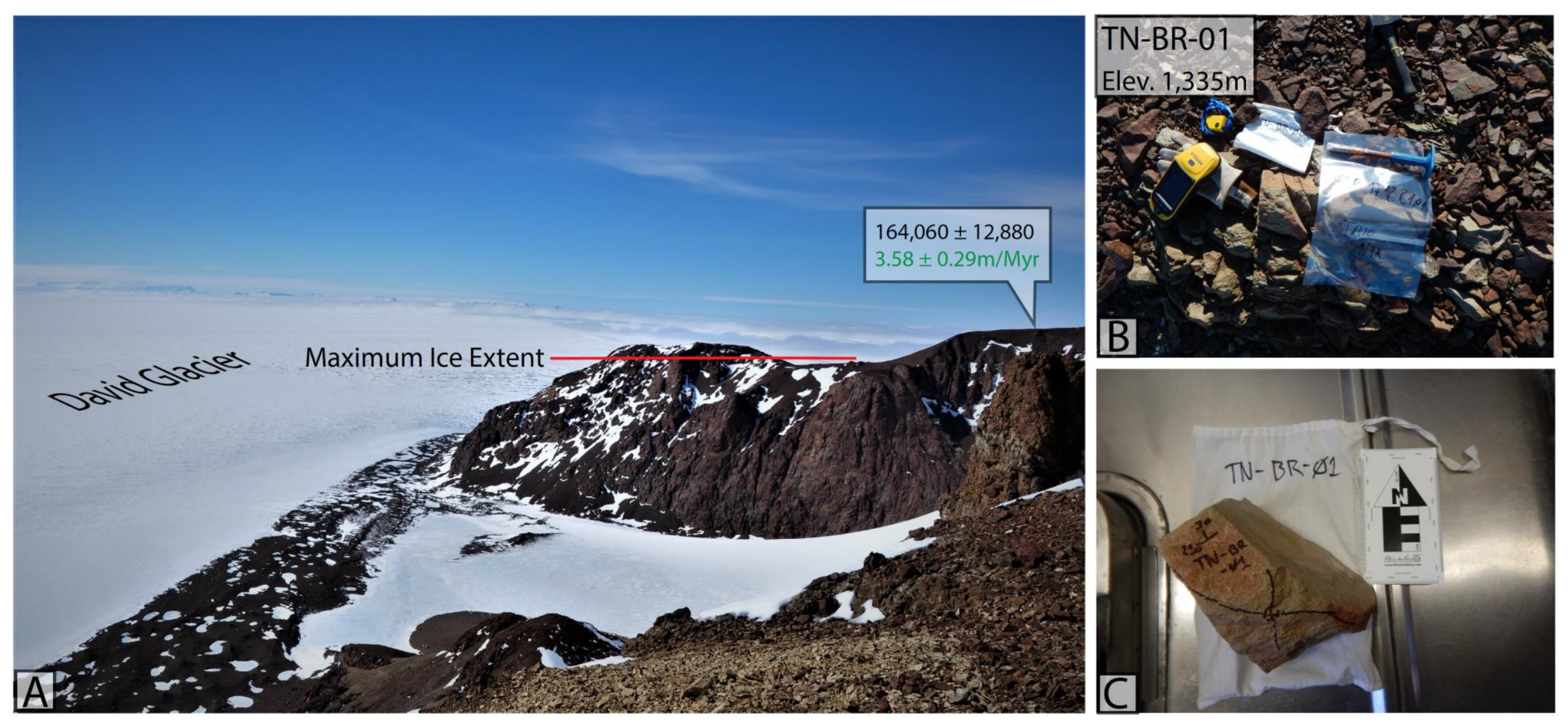

\section{Figure 7.2 West Trio Nunatak}

Profile photo looking east at West Trio Nunatak showing sample location (A) and sample outcrop (B). A photo of the sample is shown in (C). 


\subsubsection{Rock description}

The sample TN-BR-01 is a coarse-grained quartz arenite of the Beacon Super Group (Figure 7.2B). The rock is white on a fresh surface and weathers to a tan-buff colour with an $\sim 0.5 \mathrm{~cm}$ thick weathering rind (Figure $7.2 \mathrm{C}$ ). A $\sim 0.5 \mathrm{~m}$ chill margin is present at the contact of the Beacon Sandstone and Ferrar Dolorite. There are no signs of metasomatism present in the sandstone outcrop; however, the small block was subjected to high temperatures and likely magmatic fluids during the dolorite intrusion. A secondary unidentified aluminosilicate is present in the sample which hinders both the physical and chemical quartz purification processes.

\subsubsection{Bypass Ridge}

\subsubsection{Sample collection}

Two bedrock samples were collected from Bypass Ridge in November 2016 as part of a sampling campaign designed to investigate the thinning history of Tucker Glacier. Bypass Ridge is a long high ridge located at $72^{\circ} 38^{\prime} \mathrm{S} 169^{\circ} 54^{\prime} \mathrm{E}$ in the Admiralty Mountains approximately $60 \mathrm{~km}$ inland from the Borchgrevink Coast (Figure 7.1). This site was selected while on the ground because it had several areas with raised quartz veins which would have been impossible to predict using remote sensing techniques this site fortuitously proved an ideal location to compare against Cape Christie. The ridge is situated $60 \mathrm{~km}$ up Tucker Glacier and ascends from the ice surface to $\sim 660 \mathrm{~m}$ above sea level and $\sim 500 \mathrm{~m}$ above the current ice surface. The northeast ridge is sharp and moderate to steeply inclined with well exposed bedrock. Locally the bedrock is covered by a thin veneer of periglacial sediment. The sample locations span a paleo trimline of Tucker Glacier. The joint spacing is $\sim 10 \mathrm{~cm}$ with an $\sim 1 \mathrm{~cm}$ 
dry smooth gap. Secondary quartz veins exploit some joints as secondary fill and stand in relief above the greywacke bedrock (Figure 7.3B and C). 

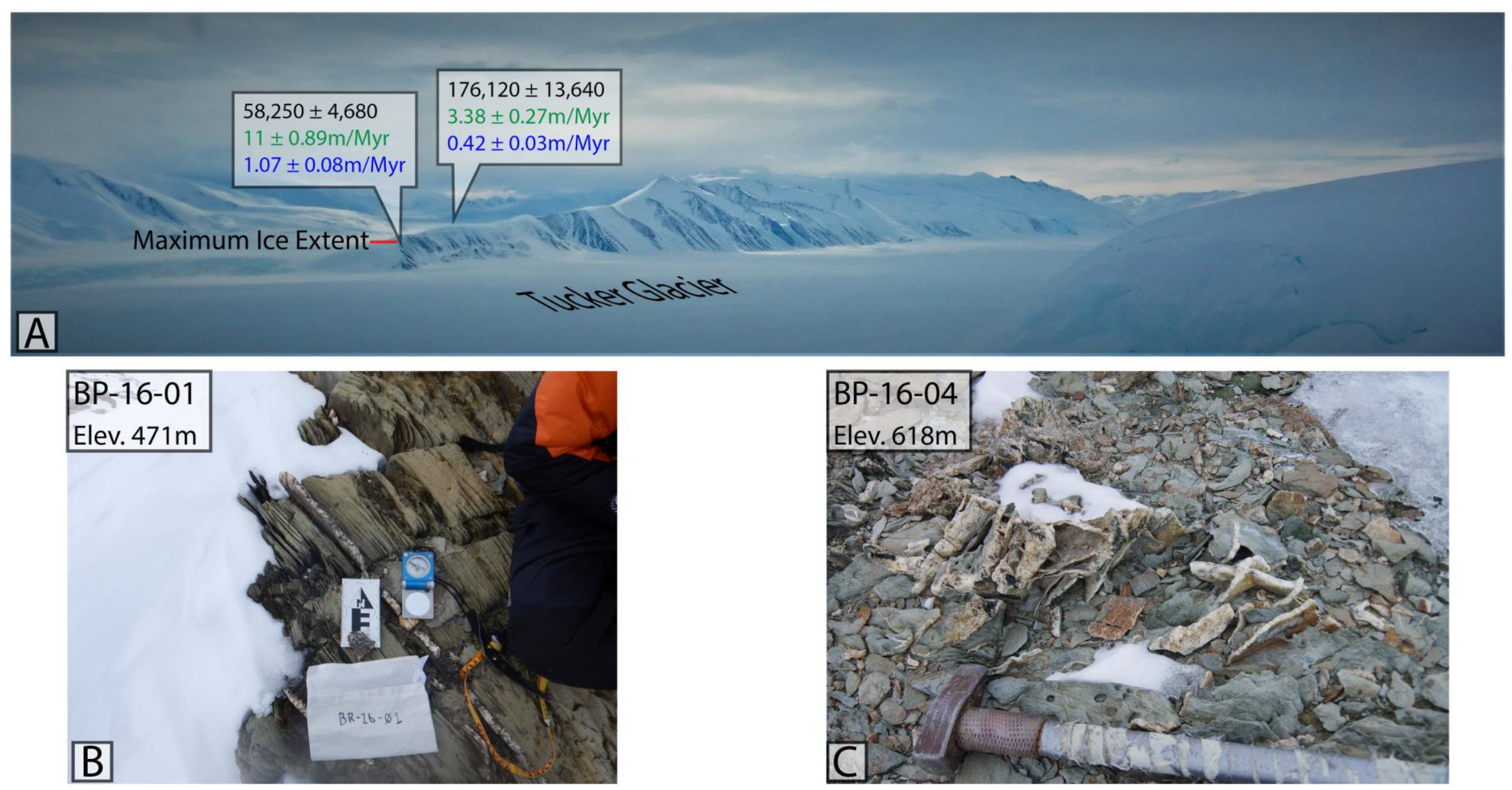

\section{Figure 7.3 Bypass Ridge}

Profile photo looking southwest at Bypass Ridge showing sample location (A) and sample outcrop for BP-16-01 (B) and BP-16-04 (C). 
Two quartz veins were collected on Bypass Ridge. The highest sample (BR-16-04) was collected at $618 \mathrm{~m}$ above sea level. This sample stood $7.5 \mathrm{~cm}$ above the surrounding greywacke bedrock that had a thin veneer of felsenmeer (Figure 7.3C). The lowest sample (BR-16-01) was collected at $471 \mathrm{~m}$ above sea level. This sample stood $6.5 \mathrm{~cm}$ above well exposed jointed bedrock (Figure 7.3B). Neither sample contained secondary minerals to interfere with the quartz purification process. The sample from $471 \mathrm{~m}$ retained striae on the top surface.

\subsubsection{Cape Christie}

\subsubsection{Sample collection}

Two bedrock samples were collected from Cape Christie in November 2016 as part of the Tucker Glacier sampling campaign. Cape Christie is a large steep peninsula located at $-72^{\circ} 17^{\prime} \mathrm{S}$ $163^{\circ} 58^{\prime} \mathrm{E}$ that extends into the Edesto Inlet (Figure 7.1). This is one of very few sample sites located on the Antarctic coast in the published literature. Much as Bypass Ridge this site was fortuitously encountered while on the ground and sampled because of several quartz veins standing in relief above the graywacke bedrock surface. The northern face of Cape Christie is steeply inclined and ascends from sea level to $\sim 920 \mathrm{~m}$ in elevation. The slope is covered with colluvium and there is a distinct absence of glacial erratics. The samples were both collected from below the paleo-trimline of a local glacier. The joint spacing is similar to the observations made at Bypass Ridge. Multiple prominent $0.5 \mathrm{~m}$ thick quartz veins were present and well exposed above the greywacke bedrock from $\sim 150 \mathrm{~m}$ to $\sim 228 \mathrm{~m}$ above sea level (Figure 7.4B and C). 

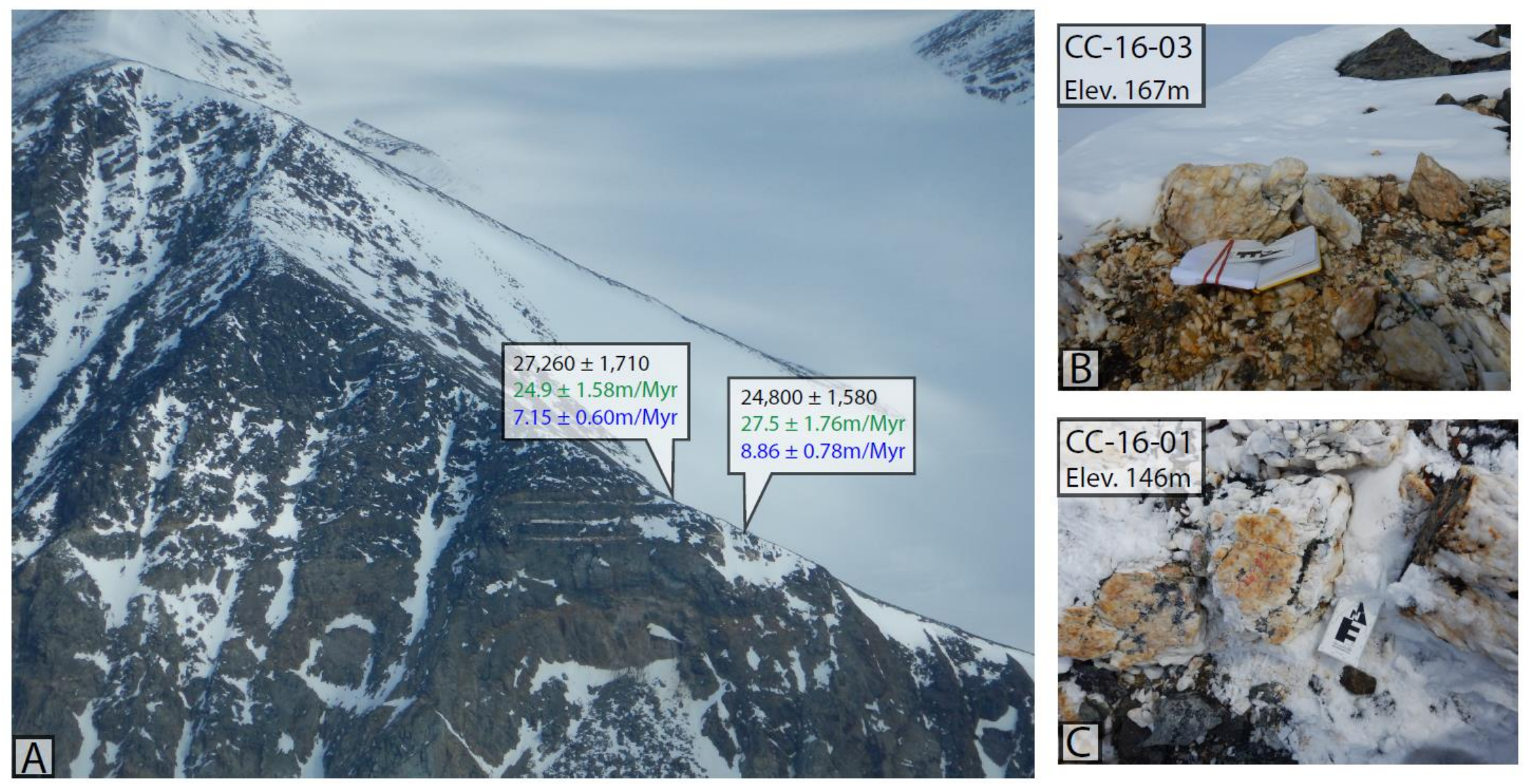

Figure 7.4 Cape Christie

Profile photo looking south at Cape Christie showing sample location (A) and sample outcrop for CC-16-03 (B) and CC-16-01 (C). 


\subsubsection{Rock description}

The two massive quartz veins were sampled on Cape Christie. The highest sample (CC-1603) was collected at $167 \mathrm{~m}$ above sea level and stood $19.5 \mathrm{~cm}$ above the bedrock surface (Figure 7.4B). The lowest sample (CC-16-01) was collected at $146 \mathrm{~m}$ above sea level and stood $22 \mathrm{~cm}$ above the bedrock surface (Figure 7.4C). Neither sample contained secondary minerals. Both quartz veins had a thin $(>0.2 \mathrm{~cm})$ brown weathering rind and glacial striae on their top surfaces.

\subsubsection{Justification for the sample sites}

The sites selected for analysis were meant for two different purposes. The differential erosion rate samples are intended as an internally consistent comparison of rock type and location. This portion of the project is intended to be directly applicable to exposure ages produces in the area (seen in Chapter 6) while also providing useful insight to the some of the potential controls on weathering and erosion. The sample collected from West Trio Nunatak was an opportunistic sample location which does reduce the size of an erosion rate data gap in the area south-west of Terra Nova Bay. The Trio Nunatak site is also useful because it shares a similar erosion rate with other locations near the sample site.

\subsection{Methods and Theory}

The general theory behind this work can be found in Chapters 1 and 2 while the general methods employed to do this work are outlined in Chapter 3. There are several caveats to discuss which build on the theory of how this work should be done that specifically relate to work in Antarctica. Additionally, due to the variety of scaling schemes in the published peer 
reviewed literature I report my cosmogenically derived erosion rates using three different scaling schemes (St, Lm, and LSDn). However, I will only discuss my results and reanalysis using the scaling scheme of Lifton and other (2014). Primarily this section is intended to review the different scenarios which impact the rate of erosion given specific paleo-environmental conditions (Kiernan et al., 2009). The first erosion framework to discuss assumes that all the inferred erosion occurred rapidly under subaerial conditions immediately after deglaciation. This requires that the newly eroded surface then remains stable enough for the accumulation of cosmogenic nuclides to accumulate. This framework assumes that the bedrock surface is in equilibrium with the rate of erosion present at the sample location. The next framework to discuss assumes instantaneous subaerial erosion just prior to the sample being collected. This requires that the surface has had a significant exposure history with no active weathering prior to the erosion event. This framework represents the most assumptions and corrections that would have to take place to produce a meaningful erosion rate (Kiernan et al., 2009). These two scenarios are in some cases warranted because evidence suggests that regions go through cycles of relatively higher and lower erosion and weathering (Sugden et al., 2005; Caraceddo et al., 2018). The third erosion rate framework to discuss is when the inferred erosion takes place over the duration of subaerial exposure. This third view provides a sight specific average erosion rate which ignores episodic periods of high and low rates of erosion. An average erosion rate is in reality a geological erosion rate and likely more useful because it describes the general rate of weathering processes over a period of time for a specific location and is useful as a correction to sample exposure ages. Additionally, applying a second nuclide that is formed in the same mineral as the primary nuclide is a way to test if the location has a simple or complex exposure history. Intervals which the ground surface is covered is shown by a difference in the overall concentration of two different nuclides. The nuclides in question can 
be two radiogenic nuclides $\left({ }^{10} \mathrm{Be}\right.$ and ${ }^{14} \mathrm{C}$ or $\left.{ }^{26} \mathrm{Al}\right)$ or a radiogenic and a stable nuclide $\left({ }^{10} \mathrm{Be}\right.$ and ${ }^{21} \mathrm{Ne}$ ). It is worth noting that the two-nuclide method is not fool proof. Caraceddo and others (2018) show that episotic erosion at high-latitude high-elevation sites in the interior of Antarctica can generate cosmogenic nuclide signatures that plot within the complex exposure domain of a multi-nuclide plot. The context for which of these approaches is most useful is topic and application dependent. There are instances when using one or the other of these approaches is warranted. For the purposes of this work I will discuss the average erosion rate for different locations in Victoria Land because it is directly relevant to exposure dating.

\subsection{Results}

\subsubsection{Explanation of overall results}

Of the five samples processed two were collected above landforms associated with glacial trimline (TN-BR-01 and BR-16-04) and three quantify the differential erosion of quartz veins and the surrounding bedrock (BR-16-01, BR-16-04, CC-16-01, and CC-16-03). The traditionally calculated erosion rates for three sample are invalid because the sample locations were overridden by glacial ice and the sampled quartz veins likely have not reached equilibrium with nuclide production and erosion (BR-16-01, CC-16-01 and CC-16-03). These three samples do retain glacial stria on their top surface which makes them ideal to investigate the differential erosion rates between the two rock types. Glacial cover complicates erosion rates because it both shields the ground surface from nuclide production and may erode several meters of bedrock. The rock surface below a glacier is shielded and may not have an exposure history when the area becomes ice free again. Therefore, the sample has not had time to equilibrate with local weathering and erosion processes which control the local rate of erosion. Samples reset by glacial processes do not represent the true erosion rate for the location or 
rock type, however, if two lithologies were eroded by the same glacier, their differential lowering can be used to determine local erosion rates.

The sample from West Trio Nunatak (Figure 7.2) was collected $\sim 60 \mathrm{~m}$ above landforms associated with a glacial trimline and it is likely that this site was free of ice for the duration of exposure. The calculated exposure age is $164,060 \pm 12,880$ years (Table 7.3) and the calculated erosion rate is $3.58 \pm 0.29 \mathrm{~m} / \mathrm{Myr}$ (Table 7.4). However, without a second nuclide to confirm that the location has had a continuous exposure history I must assume that the exposure history and erosion rate are accurate.

Two samples were collected from Bypass Ridge (Figure 7.3). Sample BR-16-04 was collected above landforms associated with a glacial trimline and has an apparent exposure age of $176,120 \pm 13,640$ years (Table 7.3) with a calculated erosion rate of $3.38 \pm 0.27 \mathrm{~m} / \mathrm{Myr}$ (Table 7.4). The differential erosion calculated for this location is $0.42 \pm 0.08 \mathrm{~m} / \mathrm{Myr}$ (Table 7.5). The second sample BR-16-01 was collected below landforms associated with a glacial trimline and has an apparent exposure age of 58,250 $\pm 4,680$ years (Table 7.1) and a calculated differential erosion of $1.07 \pm 0.08 \mathrm{~m} / \mathrm{Myr}$ for this location (Table 7.3).

Two samples were collected from Cape Christie (Figure 7.4). Sample CC-16-01 was collected from the remnant bed of a dynamic glacier the sample had well developed glacial striae oriented east-west and has an apparent exposure age of 24,800 $\pm 1,580$ years (Table 7.1) with a calculated erosion rate of $27.5 \pm 1.76 \mathrm{~m} / \mathrm{Myr}$ (Table 7.2). The critical value for this location is the differential erosion rate calculated for this sample which is $8.86 \pm 0.78 \mathrm{~m} / \mathrm{Myr}$ (Table 7.3). The second sample, CC-16-03, was collected $~ 20$ m higher than CC-16-01 and shows the same sample morphology and striae orientation. sample CC-16-03 has an apparent exposure 
age of $27,260 \pm 1,710$ years (Table 7.1) and a calculated differential erosion rate of $7.15 \pm 0.06 \mathrm{~m} / \mathrm{Myr}$ for this location (Table 7.3). 
Table 7.1 Concentrations calculations

Data to recalculate sample ages

\begin{tabular}{|c|c|c|c|c|c|c|c|c|c|c|c|c|c|}
\hline $\begin{array}{l}\text { Sample } \\
\text { Number }\end{array}$ & $\begin{array}{c}{ }^{10} \mathrm{Be} /{ }^{9} \mathrm{Be} \\
\text { Ratio }\end{array}$ & $\begin{array}{c}\text { AMS } \\
\text { Uncertainty }\end{array}$ & $\begin{array}{c}{ }^{9} \text { Be } \\
\text { STD } \\
\text { Mass(g) }\end{array}$ & $\begin{array}{l}{ }^{9} \mathrm{Be} \\
\mathrm{STD} \\
(\mathbf{p p m})\end{array}$ & $\begin{array}{c}\text { Uncertainty } \\
\text { (ppm) }\end{array}$ & $\begin{array}{c}{ }^{9} \text { Be } \\
\text { STD } \\
\text { Be (g) }\end{array}$ & $\begin{array}{c}{ }^{9} \mathbf{B e} \\
\text { Uncertainty } \\
\text { (g) }\end{array}$ & $\begin{array}{c}\text { Qtz } \\
\text { Mass (g) }\end{array}$ & $\begin{array}{c}{ }^{10} \text { Be Lab } \\
\text { Blank (at) }\end{array}$ & $\begin{array}{c}\text { Blank } \\
\text { Uncertainty } \\
\text { (at) }\end{array}$ & ${ }^{9} \mathbf{B e}$ (at) & ${ }^{10} \mathrm{Be}(\mathrm{at} / \mathrm{g})$ & $\begin{array}{c}{ }^{10} \mathrm{Be} \\
\text { Concentration } \\
\text { Uncertainty } \\
(\text { at } / \mathrm{g})\end{array}$ \\
\hline TN-BR-01 & $3.92 \mathrm{E}-12$ & $4.20 \mathrm{E}-14$ & 0.8877 & 305 & 3 & $2.707 \mathrm{E}-04$ & $2.66 \mathrm{E}-06$ & 24.2471 & $6.64 \mathrm{E}+04$ & $1.34 \mathrm{E}+04$ & $1.81 \mathrm{E}+19$ & $2.92 \mathrm{E}+06$ & $4.25 \mathrm{E}+04$ \\
\hline BR-16-01 & $1.37 \mathrm{E}-12$ & $2.01 \mathrm{E}-14$ & 0.8883 & 305 & 3 & $2.709 \mathrm{E}-04$ & 2.67E-06 & 51.6978 & $6.64 \mathrm{E}+04$ & $1.34 \mathrm{E}+04$ & $1.81 \mathrm{E}+19$ & $4.79 \mathrm{E}+05$ & $8.49 \mathrm{E}+03$ \\
\hline BR-16-04 & 4.85E-12 & 4.51E-14 & 0.8829 & 305 & 3 & $2.693 \mathrm{E}-04$ & $2.65 \mathrm{E}-06$ & 52.0201 & $6.64 \mathrm{E}+04$ & $1.34 \mathrm{E}+04$ & $1.80 \mathrm{E}+19$ & $1.68 \mathrm{E}+06$ & $2.27 \mathrm{E}+04$ \\
\hline CC-16-01 & 3.38E-13 & 7.04E-15 & 0.8848 & 305 & 3 & 2.699E-04 & $2.65 \mathrm{E}-06$ & 39.2588 & $6.64 \mathrm{E}+04$ & $1.34 \mathrm{E}+04$ & $1.80 \mathrm{E}+19$ & $1.53 \mathrm{E}+05$ & $3.59 \mathrm{E}+03$ \\
\hline CC-16-03 & $3.80 \mathrm{E}-13$ & $6.78 \mathrm{E}-15$ & 0.8857 & 305 & 3 & $2.701 \mathrm{E}-04$ & $2.66 \mathrm{E}-06$ & 40.3393 & $6.64 \mathrm{E}+04$ & $1.34 \mathrm{E}+04$ & $1.81 \mathrm{E}+19$ & $1.69 \mathrm{E}+05$ & $3.48 \mathrm{E}+03$ \\
\hline MG-16-01B & $7.58 \mathrm{E}-13$ & $1.11 \mathrm{E}-14$ & 0.886 & 305 & 3 & $2.702 \mathrm{E}-04$ & $2.66 \mathrm{E}-06$ & 51.8642 & $6.64 \mathrm{E}+04$ & $1.34 \mathrm{E}+04$ & $1.81 \mathrm{E}+19$ & $2.63 \mathrm{E}+05$ & $4.66 \mathrm{E}+03$ \\
\hline $\begin{array}{l}\text { RN85 } \\
\text { (Blank) }\end{array}$ & 3.68E-15 & 7.42E-16 & 0.8836 & 305 & 3 & $2.695 \mathrm{E}-04$ & $2.65 \mathrm{E}-06$ & 0 & $6.64 \mathrm{E}+04$ & 13373.77 & $1.80 \mathrm{E}+19$ & & \\
\hline
\end{tabular}

Table 7.2 Calculations for ages and erosion rates

Data to recalculate erosion rates

\begin{tabular}{|c|c|c|c|c|c|c|c|c|c|c|c|}
\hline $\begin{array}{l}\text { Sample } \\
\text { Number }\end{array}$ & $\begin{array}{l}\text { Latitude } \\
\text { (DD) }\end{array}$ & $\begin{array}{l}\text { Longitude } \\
\text { (DD) }\end{array}$ & $\begin{array}{c}\text { Elevation } \\
\text { (m) }\end{array}$ & ATM & $\begin{array}{c}\text { Thickness } \\
\text { (cm) }\end{array}$ & $\begin{array}{c}P \\
\left(\mathrm{~g} / \mathrm{cm}^{3}\right)\end{array}$ & Shielding & $\begin{array}{c}\text { Erosion } \\
\text { Rate } \\
(\mathbf{m} / \mathbf{M y r})\end{array}$ & $\begin{array}{c}{ }^{10} \mathrm{Be} \\
\text { Concentration } \\
\quad(\mathbf{a t} / \mathrm{g})\end{array}$ & $\begin{array}{c}{ }^{10} \text { Be Concentration } \\
\text { Uncertainty (at/g) }\end{array}$ & $\begin{array}{c}{ }^{10} \mathrm{Be} \\
\text { Standard }\end{array}$ \\
\hline TN-BR-01 & -75.471 & 19.60379 & 1335 & ant & 3.9 & 2.7 & 0.999448 & 0 & 2920021 & 42517.73 & 07KNSTD \\
\hline BR-16-01 & -72.4471 & 168.5342 & 471 & ant & 5.9 & 2.7 & 0.999026 & 0 & 479149.2 & 8488.496 & 07KNSTD \\
\hline BR-16-04 & -72.4471 & 168.5342 & 618 & ant & 1.5 & 2.7 & 0.99992 & 0 & 1677814 & 22715.18 & 07KNSTD \\
\hline CC-16-01 & -72.2776 & 169.9549 & 146 & ant & 3.1 & 2.7 & 0.99992 & 0 & 153471.4 & 3592.163 & 07KNSTD \\
\hline CC-16-03 & -72.283 & 169.9549 & 167 & ant & 4.8 & 2.7 & 0.993144 & 0 & 168561.9 & 3481.14 & 07KNSTD \\
\hline MG-16-01B & -76.1923 & 161.9415 & 252 & ant & 0.9 & 2.7 & 0.991962 & 0 & 262566.1 & 4660.54 & 07KNSTD \\
\hline
\end{tabular}


Table 7.3 Apparent exposure age values

Calculated sample ages reported in St in orange, $\mathrm{Lm}$ in green, and LDSn in blue

\begin{tabular}{llllllllllll}
$\begin{array}{l}\text { Sample } \\
\text { name }\end{array}$ & Nuclide & St & \multicolumn{3}{c}{ Lm } & \multicolumn{3}{c}{ LSDn } \\
\hline & & & & & & & & & \\
\hline
\end{tabular}

\section{Table 7.4 Erosion rate values}

Calculated sample erosion rates reported in St in orange, Lm in green, and LDSn in blue

\begin{tabular}{|c|c|c|c|c|c|c|c|c|c|c|c|c|c|}
\hline \multirow{2}{*}{$\begin{array}{l}\text { Sample } \\
\text { name }\end{array}$} & \multirow[t]{2}{*}{ Nuclide } & \multicolumn{4}{|l|}{ St } & \multicolumn{4}{|l|}{ Lm } & \multicolumn{4}{|l|}{ LSDn } \\
\hline & & $\begin{array}{l}\text { Erosion } \\
\text { rate } \\
(\mathrm{g} / \mathrm{cm} 2 / \mathrm{yr})\end{array}$ & $\begin{array}{l}\text { Erosion } \\
\text { rate } \\
(\mathrm{m} / \mathrm{Myr})\end{array}$ & $\begin{array}{l}\text { Internal } \\
\text { uncert } \\
\text { (m/Myr) }\end{array}$ & $\begin{array}{l}\text { External } \\
\text { uncert } \\
(\mathbf{m} / \mathbf{M y r})\end{array}$ & $\begin{array}{l}\text { Erosion } \\
\text { rate } \\
(\mathrm{g} / \mathrm{cm} 2 / \mathrm{yr})\end{array}$ & $\begin{array}{l}\text { Erosion } \\
\text { rate } \\
(\mathbf{m} / \mathbf{M y r})\end{array}$ & $\begin{array}{l}\text { Internal } \\
\text { uncert } \\
(\mathbf{m} / \mathbf{M y r})\end{array}$ & $\begin{array}{l}\text { External } \\
\text { uncert } \\
\text { (m/Myr) }\end{array}$ & $\begin{array}{l}\text { Erosion } \\
\text { rate } \\
(\mathrm{g} / \mathrm{cm} 2 / \mathrm{yr})\end{array}$ & $\begin{array}{l}\text { Erosion } \\
\text { rate } \\
(\mathbf{m} / \mathbf{M y r})\end{array}$ & $\begin{array}{l}\text { Internal } \\
\text { uncert } \\
(\mathbf{m} / \mathbf{M y r})\end{array}$ & $\begin{array}{l}\text { External } \\
\text { uncert } \\
(\mathbf{m} / \mathbf{M y r})\end{array}$ \\
\hline TN-BR-01 & Be-10 (qtz) & 0.000893 & 3.31 & 0.0525 & 0.289 & 0.000921 & 3.41 & 0.054 & 0.283 & 0.000966 & 3.58 & 0.0564 & 0.235 \\
\hline BR-16-01 & Be-10 (qtz) & 0.00282 & 10.4 & 0.19 & 0.869 & 0.0029 & 10.7 & 0.195 & 0.85 & 0.00296 & 11 & 0.2 & 0.694 \\
\hline BR-16-04 & Be-10 (qtz) & 0.000863 & 3.2 & 0.0473 & 0.28 & 0.000891 & 3.3 & 0.0487 & 0.274 & 0.000913 & 3.38 & 0.0498 & 0.223 \\
\hline CC-16-01 & $\mathrm{Be}-10(\mathrm{qtz})$ & 0.00704 & 26.1 & 0.617 & 2.17 & 0.00723 & 26.8 & 0.634 & 2.13 & 0.00742 & 27.5 & 0.651 & 1.76 \\
\hline CC-16-03 & Be-10 (qtz) & 0.00639 & 23.7 & 0.495 & 1.96 & 0.00656 & 24.3 & 0.508 & 1.91 & 0.00673 & 24.9 & 0.521 & 1.58 \\
\hline MG-16-01B & Be-10 (qtz) & 0.00447 & 16.6 & 0.299 & 1.36 & 0.00459 & 17 & 0.307 & 1.33 & 0.0047 & 17.4 & 0.314 & 1.09 \\
\hline
\end{tabular}


Table 7.5 Calculated greywacke differential erosion rates

Calculated differential erosion rates

\begin{tabular}{lllllll} 
Sample \# & $\begin{array}{c}\text { Height } \\
(\mathrm{cm})\end{array}$ & $\begin{array}{c}\text { Sample Age } \\
(\mathrm{yr})\end{array}$ & $\begin{array}{c}\text { Erosion } \\
\text { Rate } \\
(\mathrm{m} / \mathrm{Myr})\end{array}$ & $\begin{array}{c}\text { Internal Error } \\
(\mathrm{m} / \mathrm{Myr})\end{array}$ & $\begin{array}{c}\text { External Error } \\
(\mathrm{m} / \mathrm{Myr})\end{array}$ & $\begin{array}{c}\text { Total Error } \\
(\mathrm{m} / \mathrm{Myr})\end{array}$ \\
\hline BR-01 & 6.29 & 58200. & 1.08 & 0.09 & 0.08 & 0.09 \\
BR-04 & 7.5 & 176100 & 0.43 & 0.04 & 0.03 & 0.03 \\
CC-01 & 22.00 & 24800 & 8.87 & 0.85 & 0.71 & 0.78 \\
CC-03 & 19.50 & 27200 & 7.15 & 0.65 & 0.55 & 0.60
\end{tabular}




\subsubsection{Accelerator mass spectrometry issues}

The use of a second nuclide would constrain the ice burial history of samples TN-BR-01 and BR-16-04. However, burial ages could not be calculated due to isobaric ${ }^{26} \mathrm{Mg}$ interference in the accelerator mass spectrometer.

\subsection{Discussion of new bedrock erosion rates from Victoria Land}

The samples TN-BR-01 and BR-16-04 both consistent with the average erosion rates (3.9 $\pm 0.39 \mathrm{~m} / \mathrm{Myr}$ ) for polar climates overall (Protenga et al., 2011). However, TN-BR-01 is higher than the average sandstone specific erosion rate in Antarctica. The Antarctic average for sandstone samples is $0.52 \pm 0.36 \mathrm{~m} / \mathrm{Myr}$ (Marrero et al., 2018), though this average is heavily biased by the large number of data points from the McMurdo Dry Valleys; a region known for extremely low erosion rates. The calculated rate for TN-BR-01 agrees well with another sandstone denudation rate collected $\sim 80 \mathrm{~km}$ south at Reckling Peak (4.05 $\pm 0.69 \mathrm{~m} / \mathrm{Myr})$ in the Prince Albert Mountains (Nishiizumi et al., 1991), well away from the McMurdo Dry Valleys. No published quartz vein erosion rates exist for statistical comparison to sample BR-16-04 but this sample has a higher erosion rate than all other average lithologic specific erosion rates in the region. The likely cause is the relatively low Antarctic latitude and the maritime climate of Northern Victoria Land in the Cape Adare region.

The coastal greywacke erosion rates from Cape Christie (CC-16-01 8.86 $\pm 0.78 \mathrm{~m} / \mathrm{Myr}$, and CC-16-03 7.15 $\pm 0.60 \mathrm{~m} / \mathrm{Myr}$ ) are the highest erosion rates on in Northern Victoria Land and among the highest on the continent. The denudation rates from Cape Christie are average for global non-polar cold climates as calculated by Portenga and others (2011). These high erosion rates may be a function of lithology, joint spacing, increased freeze thaw, salt wedging, precipitation, and local humidity. 
In contrast the inland greywacke erosion rate from Bypass Ridge (BR-16-01 $1.07 \pm 0.08 \mathrm{~m} / \mathrm{Myr}$ ) represents a significant drop in lithology specific erosion rate. The main differences between Cape Christie and Bypass Ridge are elevation and proximity to seasonally open water. The dramatic difference in erosion rate implies a strong erosion gradient between coastal and inland locations. Unfortunately, the lack of appropriate resolution data sets in the area limits the use of climate models to empirically assess the dominance of climatic and geologic variable on the rate of erosion in the area.

\subsubsection{Reanalysis and interpretation of bedrock erosion rates in Victoria Land}

To complement the new erosion rate estimates from this study, I conducted a reanalysis of ten previously published bedrock erosion rates in Victoria Land north of the McMurdo Dry Valleys (Nishiizumi et al., 1986; Nishiizumi et al., 1991; Van der Wateren et al., 1999; Oberholzer et al, 2003; Welten et al., 2008; and; Di Nicola et al., 2012) (Table 7.6 and Figures 7.5-7.8 ). The selection process focused on published bedrock erosion rates produced by terrestrial cosmogenic nuclide techniques from any altitude and rock type. I omitted erosion rates measured on glacial erratics, because they violate all of the assumptions necessary to calculate erosion rates using terrestrial cosmogenic nuclides.

The reanalysis data and the new erosion rates from this study were plotted together in order to evaluate whether elevation, continentality, and latitude impact the rate of erosion in Victoria Land. The plotted data show a strong negative correlation between erosion rate and both elevation and continentality (Figures 7.5 and 7.6) while no obvious relationship with latitude exists (Figure 7.7). Due to the lack quantitative data from low elevation areas close to seasonally open water there the two low-elevation samples (CC-16-01 and CC-16-03) are critical initial steps towards filling a large data gap. 
Table 7.6 Sample reanalysis of other Victoria land erosion rates

The reanalysis data are all presented in grey and use LDSn scaling for consistency. The data from this thesis are presented in blue. All data are plotted on figures 7.5-7.8.

\begin{tabular}{|c|c|c|c|c|c|c|c|c|}
\hline Sample \# & $\begin{array}{c}\text { Elevation } \\
(\mathrm{m})\end{array}$ & $\begin{array}{c}\text { Distance from } \\
\text { Coast }(\mathrm{km}) \\
\end{array}$ & Location & $\begin{array}{c}\text { LSDn } \\
\text { Age }(\mathrm{yr}) \\
\end{array}$ & $\begin{array}{c}\text { Total } \\
\begin{array}{c}\text { Uncertainty } \\
(\mathrm{yr})\end{array} \\
\end{array}$ & $\begin{array}{c}\text { Erosion Rate } \\
\text { LDSn (m/Myr) } \\
\end{array}$ & $\begin{array}{c}\text { Total } \\
\text { Uncertainty } \\
(\mathrm{m} / \mathrm{Myr}) \\
\end{array}$ & Rock Type \\
\hline $\mathrm{O} 2003-\mathrm{K} 3$ & 1090 & 12 & Mt. Keinath BR & 2259592 & 561073 & 0.258 & 0.0743 & Granite \\
\hline DN2012-POL-3 & 2640 & 85 & Mt. Pollock & 2723119 & 556764 & 0.222 & 0.0497 & Granodiorite \\
\hline $\begin{array}{c}\text { DN2012-ARC- } \\
1\end{array}$ & 2725 & 90 & Archambault Ridge & 3350568 & 837200 & 0.19 & 0.047 & Granodiorite \\
\hline AV9008 & 1387 & 63 & Unnamed Nunatak & 383522 & 36267 & 1.47 & 0.1519 & Granite \\
\hline DW90108 & 1850 & 156 & Schroeder Spur & 995979 & 136526 & 0.55 & 0.0901 & $?$ \\
\hline DW91013 & 2850 & 156 & Mt. Burnham & 4955112 & 4645829 & 0.153 & 0.0969 & $?$ \\
\hline Dw91027 & 1420 & 54 & Mt. Billing & 523215 & 84997 & 1.05 & 0.1926 & Granite \\
\hline RKP & 2010 & 99 & Reckling Peak & 171614 & 28074 & 4.05 & 0.69 & Sandstone \\
\hline ALH85-1 & 2085 & 79 & Allan Hills & 1328550 & 278635 & 0.494 & 0.1257 & Sandstone \\
\hline W2008-1 & 2750 & 220 & Frontier Mountain & 984548 & 98132 & 0.545 & 0.065 & Granite \\
\hline TN-BR-01 & 1335 & 95 & West Trio Nunatak & 164063 & 12880 & 3.58 & 0.2914 & Sandstone \\
\hline BR-16-01 & 471 & 50 & Lower Bypass Ridge & 58258 & 4689 & 1.0789444 & 0.087407 & Graywacke \\
\hline BR-16-04 & 618 & 50 & Upper Bypass Ridge & 176125 & 13638 & 3.38 & 0.2728 & Quartz Vein \\
\hline CC-16-01 & 146 & 0.1 & Lower Cape Christie & 24807 & 2168 & 8.8684645 & 0.781022 & Graywacke \\
\hline CC-16-03 & 167 & 0.1 & Upper Cape Christie & 27262 & 2283 & 7.1528134 & 0.603228 & Graywacke \\
\hline
\end{tabular}




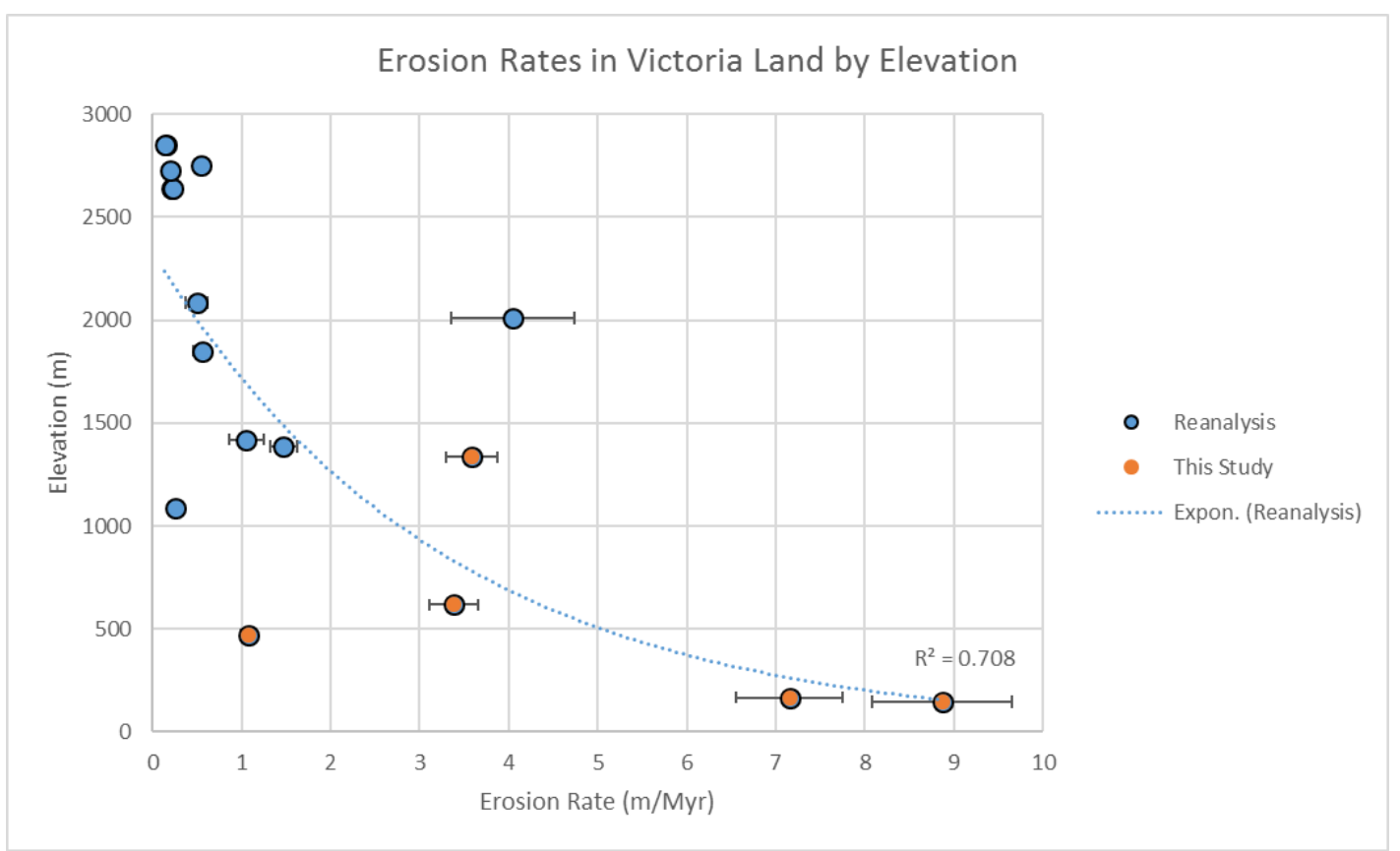

Figure 7.5 Erosion rates in Victoria Land by elevation

The data presented are for both the ten reanalysis samples located throughout high inland locations in Victoria Land (blue) and the five new samples presented in this thesis (orange). A moderate to strong negative correlation $\left(\mathrm{R}^{2}=0.708\right)$ exists between bedrock erosion rates and elevation.

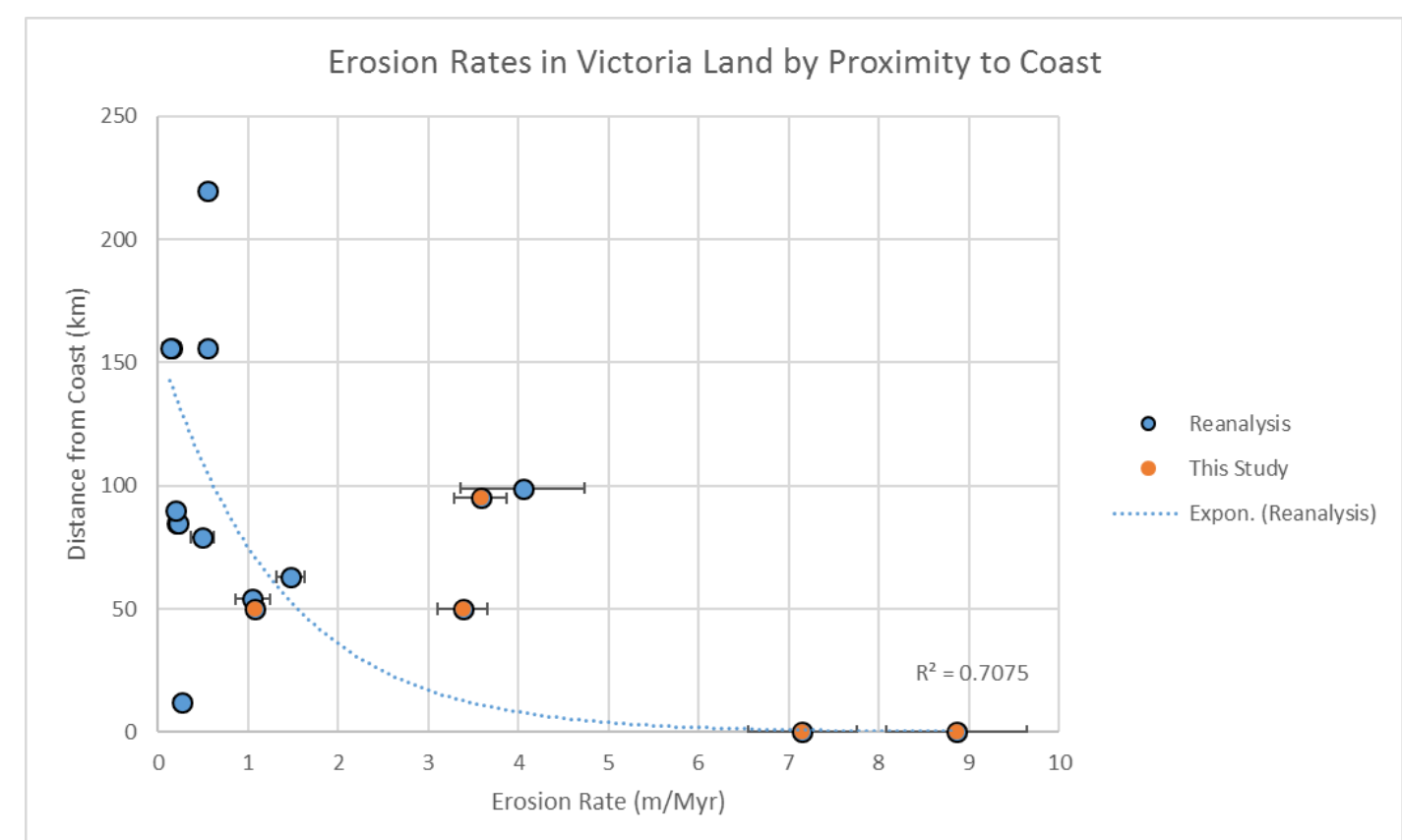

Figure 7.6 Erosion rates in Victoria Land by proximity to coast (continentality)

The data presented above are both reanalysis data (blue) and new data from this thesis (orange) comparing erosion rate to continentality. Much as the data presented in figure 7.5 , erosion rates are also show moderate to strong $\left(R^{2}=0.7075\right)$ negative correlated to proximity to the coast. 


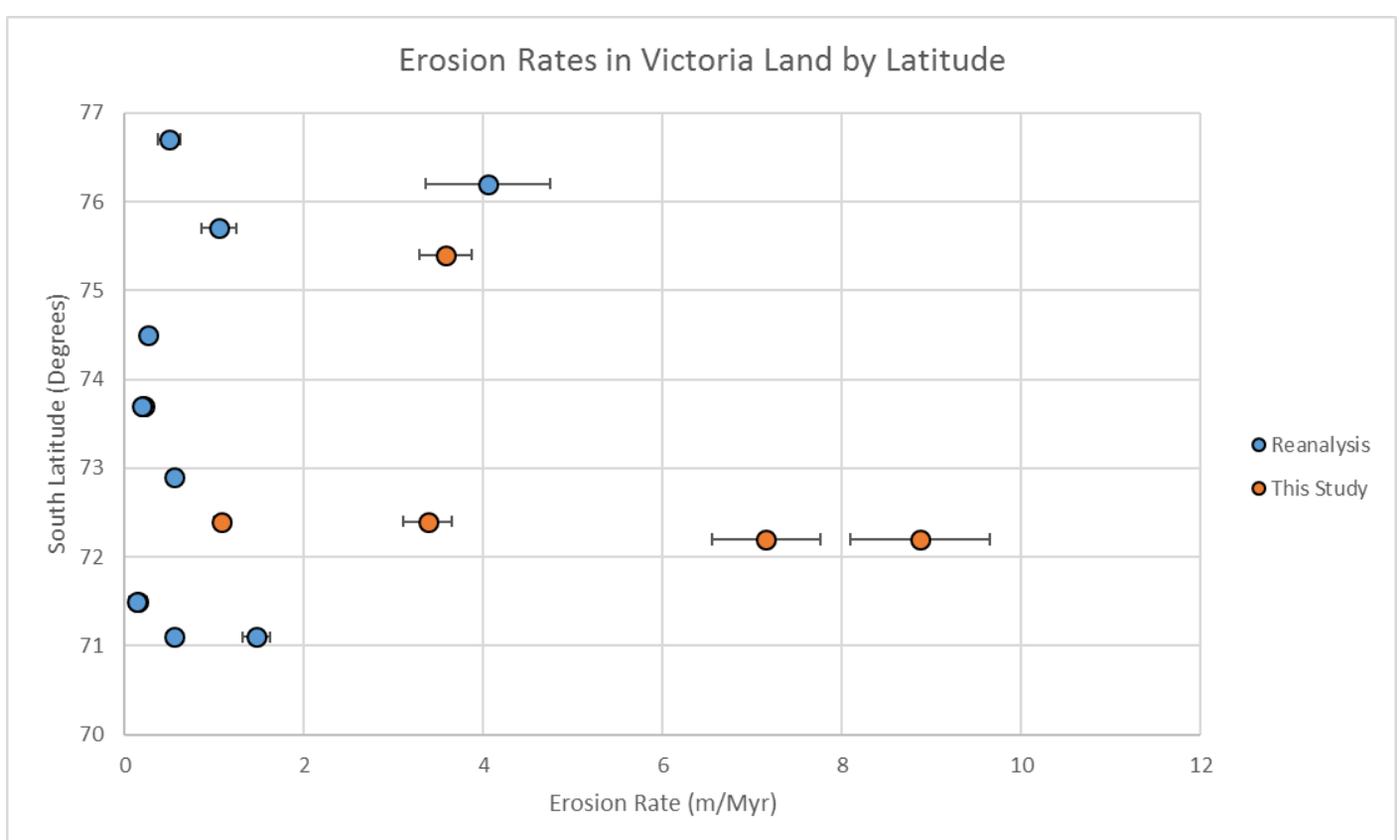

Figure 7.7 Erosion rate in Victoria Land by latitude

The latitudinal data for does not show a statistical trend. Much of the data are from inland high-altitude locations.

The newly measured bedrock erosion rates from this thesis indicate that the Admiralty Mountains experience higher rate of erosion then most other parts of Victoria Land, likely due to higher humidity and temperature in this region as well as sea salt aerosols produced by seawater. The data from Cape Christie are as high as the mean erosion rates for non-polar cold climates shown by Portenga and others (2011) while the data from Bypass Ridge are average for polar climates (Portenga et al., 2011; Marrero et al., 2018).The coastal climate may produce conditions that enable a higher rate of physical (chemically altered frost action and ice segregation) and chemical (salt weathering) weathering to take place. These physical and chemical weathering processes are likely enhanced by structural and sedimentological anisotropy (joints, metamorphic foliation, and laminae) in the greywacke bedrock. The combination of physical and chemical processes coupled with weak greywacke results in the two highest estimates of erosion rate in Northern Victoria Land and relatively high rates for Antarctica overall. 
Pervious terrestrial cosmogenic nuclide erosion rate work in the region has shown that erosion for the surrounding mountain ranges in Victoria Land vary from $1.47 \pm 0.15 \mathrm{~m} / \mathrm{Myr}$ to $0.19 \pm 0.047 \mathrm{~m} / \mathrm{Myr}$. The low bedrock erosion rates from the Deep Freeze Range and the Usarp Mountains (Figure 7.1) (Van der Wateren et al., 1999; Oberholzer et al., 2003; Welten et al., 2008; Di Nicola., 2012) are average for their rock type and typical for Antarctica in terms of elevation and proximity to the ice sheet. The six sample locations from Mount Keinath northwest to Mount Burnham occur in a region dominated by cold hyper-arid katabatic winds blowing from the Antarctic interior down the confluence zones of the Reeves Glacier in the south and the Rennick Glacier in the north (Figures 7.1 and 7.8). These two katabatic confluences funnel the cold, dry, and sediment-starved air around the Admiralty and Victory mountains (Leipzig et al., 2004; Rodrigo et al., 2012) (Figures 7.1 and 7.8). The dominant weathering process in katabatic corridors is abrasion from wind-blown snow (Marrero et al., 2018). However, ice crystals are softer than the granite and granodiorite in the sample locations, and this inefficient erosion process produces low erosion rates. Therefore, other more effective erosion and weathering processes must dominate in the Admiralty and Victory mountains.

The differential erosion rate work at Cape Christie provide geomorphic evidence of the enhanced weathering processes. I cannot unequivocally rule out that the observed erosion rates at Cape Christie are from modern erosion or past erosion on a well-preserved surface, I can only provide an average erosion rate over the last $\sim 20 \mathrm{kya}$. Additionally, the samples were collected $\sim 1 \mathrm{~m}$ from a cliff face and $\sim 100 \mathrm{~m}$ above the nearest cold-based ice lobe which makes a multiple exposure history due to cold-based glaciation unlikely, but it cannot be eliminated. Determining the true exposure history using ${ }^{14} \mathrm{C}$ as a second cosmogenic nuclide would rule out whether this location was covered at any point in the past 20 kya. While in the field I saw 
no evidence of non-dynamic ice cover in the area around the samples. The timing of erosion cannot be determined which means that I cannot compare the Cape Christie samples to an active erosion study in the Larsemann and Vestfold hills (Spate et al., 1995). Spate and others (1995) use a micro-erosion meter to show that the active erosion rates in a katabatic corridor near seasonally open water are between $15 \mathrm{~m} / \mathrm{Myr}$ and $22 \mathrm{~m} / \mathrm{Myr}$. What is not clear from this work is if the extremely high observed rate is the long-term average or a simply a period of high erosion in an otherwise low erosion system. Therefore, the new data sets presented in this chapter and the published rates from Spate and others (1995) cannot be compared. Additionally, work completed by French and others (1999) in the Terra Nova Bay area show extremely high erosion rates for wind abrasion (10-30 m/Myr) and taffoni development (20$30 \mathrm{~m} / \mathrm{Myr}$ to $80-100 \mathrm{~m} / \mathrm{Myr}$ ). This data set is based on the weathering of glacial erratics and exposed bedrock standing in relief on a sediment laden surface in a katabatic corridor close to the coast. Again, the erosion rates are not directly comparable because the Terra Nova Bay sample sites are in a katabatic corridor with persistent wind speeds of $\sim 35 \mathrm{kph} 50 \%$ of the time and $70 \mathrm{kph} 20 \%$ of the time (French et al., 1999) and an ample sediment supply. Cape Christie does not see sustained winds of that magnitude; therefore, the erosion rate should be lower. The Larsemann/Vestfold Hills and the Terra Nova Bay areas are good analogues to the Cape Christie sample site because they share a common variable, proximity to seasonally open seawater. Salt aerosols from ocean spray can travel inland tens of kilometres and produce salt crystals on rock surfaces (Gore et al.,1996). When salt crystals grow, they exert force on the surrounding rock and they thermodynamically reduce the melting point of any snow that comes into contact. By reducing the melting point of snow there may be an increase in the frequency of freeze thaw cycles which will enhance physical weathering. 
The group of erosion rates from West Trio Nunatak south to the Allan Hills in the Prince Albert Mountains are largely outside of the katabatic corridor of the Reeves Glacier as well, but still have relatively strong winds as compared to the Admiralty and Victory mountains (Figure 7.8). The erosion rates from West Trio Nunatak, Mount Billing, and Reckling Peak in the Prince Albert Mountains are average for polar climates (Portenga et al., 2011; Marrero et al, 2018). These sample sites are at relatively low elevation which may experience seasonal temperatures high enough to produce liquid water. If so seasonal ice wedging cycles and salt weathering may be the primary physical and chemical weathering mechanisms (French, 2007). While the low bedrock erosion rate from the Allan Hills is likely due to the extremely cold and dry climate at high elevation close to the East Antarctic Ice Sheet similar to the locations in the Deep Freeze Range and USARP Mountains (Figures 7.1 and 7.8).

The data from Cape Christie and Bypass Ridge suggest the presence of a strong erosional gradient from the coast inland and from low elevation to high elevation. The $96 \%$ reduction in erosion rate over $50 \mathrm{~km}$ is dramatic and has not been documented in this area previously. The bedrock erosion rates in Victoria Land are predominantly from granite with a few quartz rich sandstones from north of the McMurdo Dry Valleys (Figure 7.8) (Nishiizumi et al., 1986; Nishiizumi et al., 1991; Van der Warteren et al., 1999; Oberholzer et al., 2003; Welten et al., 2008; Oberholzer et al., 2008; Di Nicola et al., 2012). The bedrock erosion rates in this area are variable and scattered in the Transantarctic Mountains. The samples collected from Cape Christie have the highest erosion rates in Northern Victoria Land and are among the highest in Antarctica. This is likely due to their proximity to the moderating and humidifying effects of the ocean and salt aerosols produced by seawater. Unfortunately, my data set is limited when it comes to an overall discussion of the importance of rock type. However, it is worth noting that the erosion rates at a moderate elevation away from the ice sheet are at the lower boundary 
of more typical erosion rates for granite and sandstone near an ice sheet at significantly higher elevation and that the Upper Bypass Ridge sample is approximately the average polar erosion rate globally. Globally the highest erosion rates are found in humid environments with an average temperature near $10^{\circ} \mathrm{C}$. The differential erosion rates for sample CC-16-01 and CC16-03 $(8.86 \pm 0.78 \mathrm{~m} / \mathrm{Myr}$, and $7.15 \pm 0.60 \mathrm{~m} / \mathrm{Myr})$ are average for non-polar cold climates ( 7 m/Myr) (Portenga et al., 2011). 


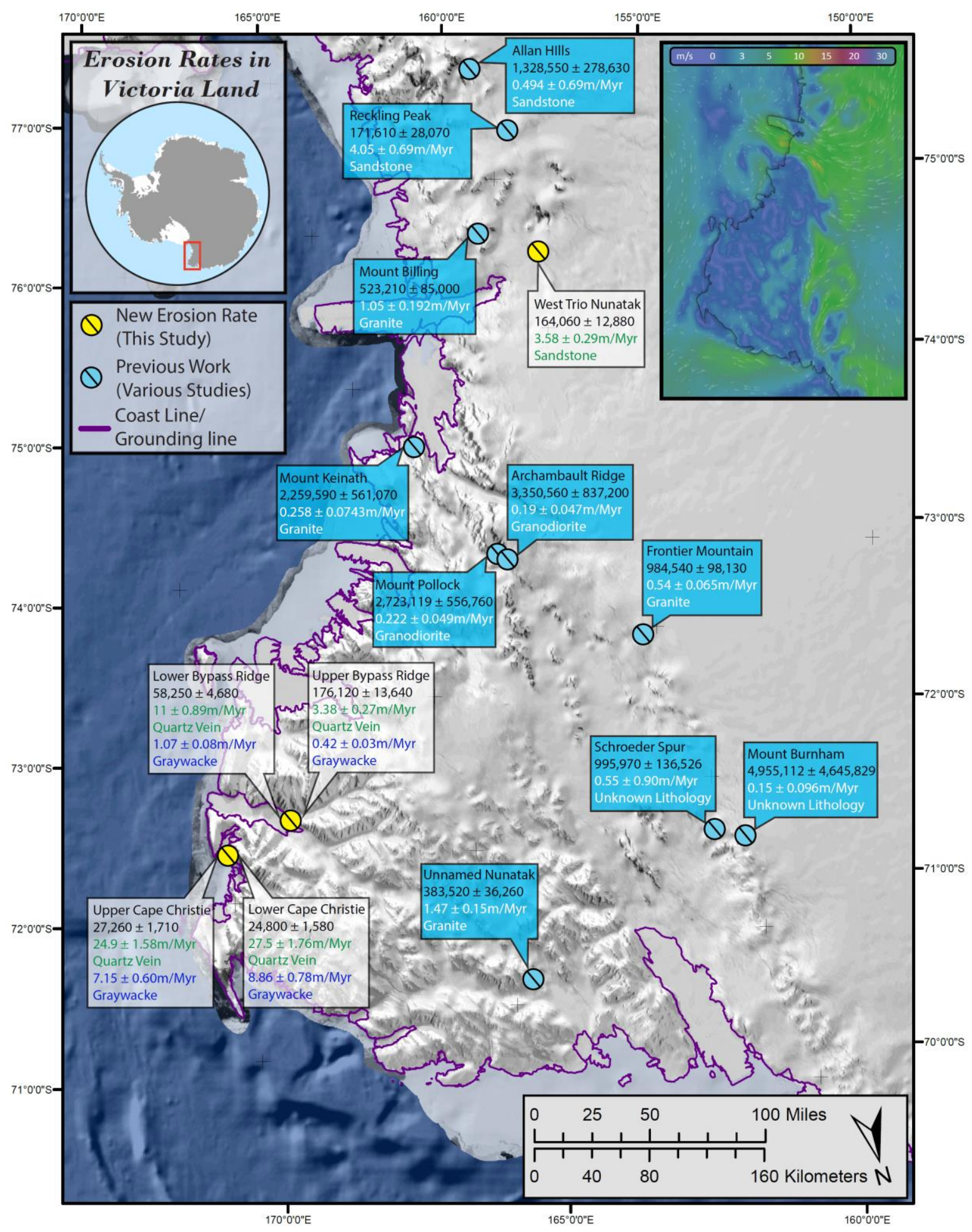

Figure 7.8 Victoria Land erosion rates

New and existing erosion rate data are presented along with the sample location, lithology and recalculated age (LDSn Lifton et al., 2014). The reanalysis data are in blue and the new data is presented in white. The low erosion rates from Mount Keinath northwest to Mount Burnham are high elevation sites in katabatic confluence zones (upper right inset map) where the dominant weathering if likely from blowing snow. The wind direction and velocity data are from windy.com. The colour scale is in $\mathrm{m} / \mathrm{s}$ ranging from $0-30$. The new data are located in areas which seasonally experience relatively warmer and more humid conditions. 


\section{$\underline{7.5 .2}$ Implications of assuming incorrect erosion rates}

Inaccurate assumptions about the rate of erosion can lead to errors when calculating exposure ages. I show that a sample age can be under or overestimated by up to $12 \%$ (Table 7.7). Table 7.7 was made using sample HP-16-11 to calculate exposure ages with no erosion and an appropriate upper limit of $8 \mathrm{~m} / \mathrm{Myr}$. This work suggests that more samples need to be collected to better constrain erosion rates along coastlines with close proximity to seasonally open water and away from katabatic corridors.

\section{Table 7.7 Age error from erosion rate}

The exposure age for sample HP-16-11 is calculated with zero erosion (top) and with an erosion rate of $0.0008 \mathrm{~cm} / \mathrm{yr}$ (8 m/Myr). Sample exposure ages used LDSn scaling

\begin{tabular}{|c|c|c|c|}
\hline Field Number & $\begin{array}{c}\text { Erosion Rate } \\
(\mathrm{cm} / \mathrm{yr})\end{array}$ & $\begin{array}{c}\text { Age } \\
(\mathrm{Yr})\end{array}$ & $\begin{array}{c}\text { Total Error } \\
(\mathrm{Yr})\end{array}$ \\
\hline HP-16-11 & 0 & 22383 & 2070 \\
\hline HP-16-11 & 0.0008 & 26597 & 2964 \\
\hline
\end{tabular}




\section{CONCLUSIONS}

\subsection{Quartz Purification Procedure}

1. Can a protocol be developed to improve quartz purification procedures for very finegrained quartz rich rocks?

The work presented in chapter 4 on quartz purification, both expands the rock types which can produce reliable terrestrial cosmogenic results and quantifies the timing of cation removal during quartz purification. I have quantified the minimum amount of material which needs to be removed to ensure that all meteoric ${ }^{10} \mathrm{Be}$ has been removed from a sample with a native grain size between $125 \mu \mathrm{m}$ and $65 \mu \mathrm{m}$. The purification process shows a systematic and significant reduction of $\mathrm{Fe}, \mathrm{Mg}, \mathrm{Ca}, \mathrm{Mn}, \mathrm{Al}, \mathrm{Ti}, \mathrm{K}, \mathrm{Na}$, and meteoric ${ }^{10} \mathrm{Be}$. Critically, this work also shows that fine-grained samples require more leaching (35\% mass loss) than coarse grained samples (20\% mass loss) in order to remove excess ${ }^{27} \mathrm{Al}$ (Figures 4.21 and 4.22 ). The increased amount of ${ }^{27} \mathrm{Al}$ in fine grained material was noted in foundational work compiled by Brown and others (1991) and Kohl and Nishiizumi (1992) and may prove to be a greater purification challenge than removing meteoric ${ }^{10} \mathrm{Be}$.

\subsection{Mawson and Tucker Glaciers Thinning Histories}

2. What is the magnitude, rate, and timing of ice-surface-elevation changes at Mawson and Tucker glaciers since the Last Glacial Maximum?

The work presented in chapters 5 and 6 on outlet glacier surface elevation reconstructions shows that both Mawson and Tucker glaciers responded differently to environmental forcing since the Last Glacial Maximum. At Mawson glacier, a minimum of $\sim 250 \mathrm{~m}$ of surface lowering occurred between $\sim 6.5$ kya and 4.9 kya, implying a time averaged thinning rate of 
$2.76 \mathrm{~m} \mathrm{yr}^{-1}$. The abrupt style, rapid rate, total magnitude, and timing of thinning at Mawson Glacier is similar to the thinning of Mackay Glacier $100 \mathrm{~km}$ south (Jones et al., 2015) and the thinning of David Glacier $100 \mathrm{~km}$ north (Stutz et al., in prep.). The observed outlet glacier thinning the result of Marine Ice Sheet Instability initiated by Mawson Glacier's grounding line moving off a sill and producing a positive feedback which sustained rapid thinning for thousands of years.

At Tucker Glacier where at least 300 m of thinning occurred between $\sim 19.3$ kya and $\sim 5.1$ kya, the time-averaged estimate of thinning (0.01-0.05 $\left.\mathrm{m} \mathrm{yr}^{-1}\right)$ is much lower than that at Mawson Glacier. The gradual rate and protracted nature of thinning at Tucker Glacier is dissimilar to other outlet glaciers along the Victoria Land Coast. The style of thinning at Tucker Glacier suggests that it was not confluent with grounded ice in the Ross Sea. Two other possible configurations of features and forcings may explain the rate and style of thinning: 1) a thin ice shelf may have slightly buttressed Tucker Glacier allowing it to thicken by $\sim 300 \mathrm{~m}$, then subsequent collapse would have initiated thinning, or 2) a warming ocean and rising sea level produced steady grounding line retreat and thinning over this time. Modelling this system may provide some clarity on what combination of features and forcings could reproduce the observed style of thinning.

\subsection{Bedrock Erosion Rates in Victoria Land}

\section{How does latitude, altitude, and continentality affect bedrock erosion rates in}

\section{Antarctica?}

The work presented in chapter 7 on Victoria Land erosion rates shows there is likely a strong erosion rate gradient from the coast inland and from low to high elevation. The differential erosion rate of greywacke at Cape Christie, along the coast, is the highest reported in the region 
and is relatively high for Antarctica $(8.86 \pm 0.78 \mathrm{~m} / \mathrm{Myr}$ and $7.15 \pm 0.6 \mathrm{~m} / \mathrm{Myr})$, while $50 \mathrm{~km}$ inland, at Bypass Ridge the differential erosion rate is dramatically lower $(1.07 \pm 0.08 \mathrm{~m} / \mathrm{Myr}$ and $0.42 \mathrm{~m} / \mathrm{Myr}$ ). The coastal erosion rate for both samples are average for non-polar cold climates (Protenga et al., 2011) while both inland rates are below average for polar climates (Portenga et al., 2011; Marrero et al., 2018). The cosmogenically calculated erosion rates for Upper Bypass Ridge $(3.38 \pm 0.27 \mathrm{~m} / \mathrm{Myr})$ and West Trio Nunatak $(3.58 \pm 0.29 \mathrm{~m} / \mathrm{Myr})$ are average for polar erosion rates.

\subsection{Impact of Results}

This study aims to illuminate three areas of distinct interest. The following section enumerates the potential impact of each advancement made by the completion of this work.

Firstly, it quantifies leaching procedures for rock types with fine-grain quartz. The purification procedure expands the type of rocks that can be utilized for terrestrial cosmogenic studies. This procedure is broadly applicable for rock types found all over the world. Finally, by documenting major element, ${ }^{10} \mathrm{~B},{ }^{9} \mathrm{Be}$, and meteoric ${ }^{10} \mathrm{Be}$ removal throughout the sample leaching process it quantifies standardized procedures that assume purification is complete once a specific mass loss is achieved.

Secondly, it illuminates the response of Mawson and Tucker glaciers, two large outlet glaciers in Victoria Land, to climatic and non-climatic changes since the Last Glacial Maximum. The data provided will serve to further several different scientific goals. It will provide data sets to help constrain numerical model conditions in an area that is thought by some to be one of the sources of Meltwater pulse-1a and constrain the extent of grounded ice in the Western Ross Sea since the Last Glacial Maximum. These data will also bolster the current understanding of outlet glacier response over millennial timescales. It will also provide new ice sheet histories 
to support a Victoria Land correction for the Glacial Isostatic Adjustment and the GRACE Satellite System

Thirdly, these data provide new insight to erosion rates and the landscape evolution of Victoria Land, Antarctica, a region where limited erosion rate and landscape evolution work has previously been done. This study provides high differential erosion rates for greywacke in Northern Victoria Land. Appropriate consideration of clast and bedrock erosion rates is vital for accurately calculating exposure ages for glacial thinning histories and other applications. Finally, this study helps constrain the local maximum thickness of glacial ice. 


\section{FUTURE WORK}

This section highlights potential avenues for future work as informed by this thesis.

\subsection{Removal of Laurylamine from quartz samples}

A timely extension of the quartz purification method development (Chapter 4) is to explore an effective method to remove the organic compound Laurylamine (doedecylamine) from post froth float quartz separates. Froth flotation is a widely used effective method to separate quartz from other silicate minerals, mainly feldspars and micas. The organic compound Laurylamine $\left(\mathrm{C}_{12} \mathrm{H}_{27} \mathrm{~N}\right)$ is critical for the mineral separation procedure. However, if the sample is measured for in-situ ${ }^{14} \mathrm{C}$ the Laurylamine produces erroneously old exposure ages, because the long hydrocarbon chain contains modern ${ }^{14} \mathrm{C}$ which, if analysed, distorts the ${ }^{13} \mathrm{C} /{ }^{14} \mathrm{C}$ ratio and results in an erroneously old exposure age (Nichols and Goehring, 2019). Laurylamine is insoluble in water so the current solution is to aggressively etch quartz samples with HF to remove any excess Laurylamine residue on the mineral grain and in small fractures or pits on the mineral surface (Nichols and Goehring, 2019). Aggressive etching with HF limits the type of samples which can be analysed (e.g. it cannot be used for fine-grained material and quartz poor rocks with low sample yield). However, a potential solution exploits the miscibility of Laurylamine in Ethanol $\left(\mathrm{C}_{2} \mathrm{H}_{6} \mathrm{O}\right)$. two prolonged $(24 \mathrm{hr})$ Ethanol leaches may be an appropriate purification measure for quartz before graphitizing the sample. Critically, Ethanol is miscible in water and any residual Ethanol remaining on a quartz grain can be washed off with water. This work would entail six identical sample splits from the same froth floatation batch. The first sample would remain untreated, while a second sample would only be treated with $\mathrm{HF}$ to remove $50 \%$ of its initial mass, The four remaining samples would be treated with two $24 \mathrm{hr}$ Ethanol baths. Of the samples treated with Ethanol, one sample would not be treated 
with HF while the remaining three should be leached with HF in order to remove $20 \%, 35 \%$, and $50 \%$ of the initial sample mass. While repeat in-situ ${ }^{14} \mathrm{C}$ measurements are expensive this work is critical because $i n$-situ ${ }^{14} \mathrm{C}$ is a significant advancement in the terrestrial cosmogenic field.

\subsection{Ice surface reconstructions: Expanding the work presented}

The highest priority thinning history work following this thesis is to refine the ice surface reconstruction of Tucker Glacier using in-situ ${ }^{14} \mathrm{C}$. The use of $i n-$ situ ${ }^{14} \mathrm{C}$ on eight samples with complex exposure histories from Heff's Manor should provide a robust ice surface reconstruction for future numerical modelling experiments. Additionally, a ${ }^{10} \mathrm{Be}$ ice surface reconstruction was attempted for Ironside Glacier $\sim 50 \mathrm{~km}$ north of Tucker Glacier. The results of this work did not appear in this thesis because of a lack of adequate age control; ten of the eleven samples have complex exposure histories. These samples would also benefit from insitu ${ }^{14} \mathrm{C}$ analysis. This work would provide another vital outlet glacier thinning history to Northern Victoria Land.

A direct continuation of ice surface reconstruction work that I have carried out in Northern Victoria Land would be to extend this work to Lillie and Rennick glaciers on the Oates Coast. This would provide constrains on the thinning history of outlet glaciers adjacent to the Wilkes subglacial basin. The Rennick Glacier is the largest outlet glacier in Northern Victoria Land, and drains both mountain glaciers from the Admiralty Mountains and Talos Dome, an ice dome connected to the East Antarctic Ice Sheet. This glacier is critical to understand because it was not confluent with grounded ice in the Ross Sea, which means that it should only respond to external mass balance controls (Mayewski, 1975; Mayewski et al., 1979, Denton et al., 1986; Meneghel et al., 1999). The Usarp Mountains on the western flank of Rennick 
Glacier are the closest nunataks to the Wilkes subglacial basin. Glaciers in the Wilkes subglacial basin are grounded below sea level and sit on retrograde bedrock slopes and are potentially susceptible to Marine Ice Sheet Instability (Mengel and Levermann, 2014; Golledge et al., 2015).

The Lillie Glacier is important because it shares similar basin and mass balance characteristics with Tucker Glacier. Lillie Glacier is a large alpine glacier draining from the Admiralty Mountains to the northwest and is adjacent to the Rennick Glacier. Lillie Glacier was unlikely to have been confluent with grounded ice in the Ross Sea at the Last Glacial Maximum so it may provide context to the thinning history of Tucker Glacier. If the two ice surface reconstructions were similar it would support the hypothesis that Tucker Glacier was not confluent with grounded ice in the Ross Sea.

\subsection{Erosion Rate Work: The need for further data}

The samples I collected and processed from Upper Bypass Ridge and West Trio Nunatak should be analysed for the stable isotope ${ }^{21} \mathrm{Ne}$. This should resolve whether the sample locations have a simple or complex exposure history. I tried to evaluate the exposure history using ${ }^{26} \mathrm{Al}$, however, as outlined above the samples failed while being analysed by an accelerator mass spectrometer.

The logical extension of the erosion rate work presented in this thesis falls into two categories: 1) systematic studies and 2) opportunistic studies. The systematic work should be a spatial transect which targets nunataks from the coast inland up to $50 \mathrm{~km}$. The aim of this would be to test my hypothesis that there is an erosion rate gradient. For this type of systematic sampling effort, the sampling interval should be as tight as possible from $1 \mathrm{~km}$ to $5 \mathrm{~km}$ for the first $20 \mathrm{~km}$, then every $10 \mathrm{~km}$ to $15 \mathrm{~km}$ up to $50 \mathrm{~km}$ from the coast in order to cover the most under 
represented bedrock erosion rate sampling areas. The opportunistic work should be undertaken by field parties when appropriate conditions are encountered. Both systematically and opportunistically collecting appropriate material for analysis, will lead to a more complete understanding of erosion rates in this region and Antarctica as a whole.

\subsection{Modelling of Tucker Glacier}

As mentioned in the Tucker Glacier Discussion section, a finite element model with variable resolution mesh, such as Ùa (Gudmundsson et al., 2012), could be used to evaluate the forcings and internal responses that caused the gradual thinning of Tucker Glacier as shown by the age-elevation profiles from Heffs Manor and Shark Fin. Ùa is a two-dimensional (plan form) model and has a variable resolution mesh which would capture the effects of changing valley width, glacier confluences and the grounding line in sufficient detail. A full Stokes model such as Elmer Ice (Le Meur et al., 2004) could also be utilised, but Ùa is computationally efficient and thus more suited to the many repeat experiments that would be required.

Additionally, the basin under the modern Tucker Ice Shelf has a retrograde bed slope that could initiate marine ice sheet instability but, as demonstrated in this thesis, the ice surface reconstruction does not show evidence of rapid thinning. There are several potential reasons why Tucker Glacier did not experience Marine Ice Sheet Instability during retreat. These could be explored by attempting to answer the questions below in a series of glacier modelling experiments.

- Can increased accumulation at Tucker Glacier balance the effect of thermal erosion at the grounding line given various ice sheet configurations (ice-sheet buttressed, iceshelf buttressed, and unbuttressed)? 
- How does isostatic rebound effect Tucker Glacier's grounding line once retreat is initiated? 


\section{Appendix A: Standard Physical Processing Methods}

\subsection{Cutting}

Make sure to turn both the upper and lower switches on.

Make sure you have the water faucet turned on all the way.

Adjust the amount of water at the saw (red knob).

Cut above any line that looks like it could be till related.

Cut off parts that look like they were removed post deposition

Cut to a max top to bottom thickness of $10 \mathrm{~cm}$ (Highest concentration of Be will be in this zone of the sample)

Make sure to measure the thickness of the samples as you are processing and find the average thickness.

For the granites at Bruce Point Rich has been cutting samples about 5-7 $\mathrm{cm}$ thick

Want 60-70 grams of quartz at the end

Make sure to grade the samples that are going to be used on an A, B, C... scale.

Cut the samples to a width of about your pinkie or a little larger. (They need to fit into the Boyde Crusher)

Put sample in oven to dry for 2 hours

Bags are the cabinets in the room adjacent room the saw is in.

\section{Tips:}

You do not want water to come out of the saw basin so be aware of that at all times.

Have a hammer and wide chisel with you it may be advantageous to split samples part way through cutting. (Should really only do this if you have a strip of uncut rock less than an inch thick.)

If the saw switches off the engine is probably overheated. Let it sit and cool down for between 5-15 minutes. Then press the red reset button on the top of the engine. You should hear or feel a pop. The saw should start again.

If that doesn't work you may have to reset the fuse. The fuse box is located to the right of the lab entrance. Make sure you flip the correct fuse.

$\underline{10.1 .1} * *$ Cleaning the Saw

Wash the tray down

Wash the tray cradle area down (try to remove any rock fragments found there.)

Wash the fine particulate down the drain and rinse sides of larger basin containing the saw assembly. 


\subsection{Crushing}

Turn on air outside lab turn on air inside lab (check vents in mill lab and make sure they are closed)

Turn on machine at the wall and behind the machine

Crush and slowly adjust to finer and finer size fraction

Clean between each sample (Vacuum the machine out, blow compressed air through the machine and make sure to clean the crushing plates off with an ethanol soaked paper towel and use pipe cleaners to clean hard to reach corners in the jaws of the crusher)

Hand sieve samples for medium sand fraction (250-500 microns)

*Thoroughly clean after you have finished for the day*

**Make sure that you have turned off the Boyde Crusher, Niederman extractor fan, and the air outside the crush lab before you leave**

\section{Tips:}

Be mindful of how much sample you are totally pulverizing and if that matters for the sample you are processing. (Remember you can always mill the residual material)

When sieving make sure to dump out the base frequently.

Place the extractor fans precisely, one at the lid of the crusher and one at the tray section.

Keep the extractor fan running for as long as you are in the room.

Make sure to always have a mask on even if you are not crushing samples.

$\underline{10.2 .1} * *$ Cleaning the Rock Crusher

Clean plates from above and below with vacuum, compressed air, and ethanol on paper towels.

Clean the cracks between the crush plates and the side wall with small brush and/or ethanol soaked paper towel.

Clean flaps with vacuum remove as much dust as possible

Vacuum and blow out area behind plates

Clean under motor both front and back

Clean grooves on the motor

Clean all flat surfaces inside and outside of the main box on the Boyde Crusher

Clean the joints, grooves, and open spaces around the inside edge of the main box

Wipe down all surfaces around lab with water and paper towel

Wash crush buckets in the wet room

Rotate the Niederman intensly for 1 minute or until you don't see material coming out the bottom of the extraction system (this may be for longer than one minute). 


\subsection{Milling}

Make sure to turn on in both locations (wall and behind machine).

Use the Tungsten Carbide mill (Kevin confirmed this, it will not mess up the chemistry and it is best to not touch the agate mill at all)

Put an equal amount of sample in the each ring of the mill (Use folded paper and dump sample on the middle of the large ring it should split about equally)

Run mill on low setting 750 RPM (on front of machine click dial to the left)

Make sure to place spacer in for the Tungsten Carbide mill

Tighten the screw down until hand tight be careful not to over tighten the mill ( 1/4$1 / 3$ of a turn).

Be careful with how much material you put in. The amount of material effects the amount of time you need to mill the sample. Never put anything less than $10 \mathrm{~g}$ in this mill. **Times may not be appropriate for the agate mill (need to test) $* *$

\begin{tabular}{|l|l|}
\hline $\begin{array}{l}\text { Amount of } \\
\text { sample }\end{array}$ & Duration \\
\hline $50 \mathrm{~g}$ & $4 \mathrm{sec}$ \\
\hline $100 \mathrm{~g}$ & $5 \mathrm{sec}$ \\
\hline $150 \mathrm{~g}$ & $6 \mathrm{sec}$ \\
\hline $200 \mathrm{~g}$ & $7 \mathrm{sec}$ \\
\hline
\end{tabular}

\section{Tips:}

**Make sure to sieve between each run. That way you can maximize your sample load and process results. Sieves to use 710, 500, and 250.

Your sample will probably break into $\sim 1 / 3$ increments (i.e. 1/3 710-500, 1/3 500-250, and $\sim 1 / 3$ $<250)$

Samples look slightly more rounded under a microscope.

\section{$\underline{\text { 10.3.1 }} * *$ Cleaning the Ring Mill}

To clean: wash mill with ethanol-soaked paper towels.

Blow out ring mill with compressed air

Make sure to vacuum up any material that has fallen on the outside of the machine.

Wipe down all benches and flat surfaces. 


\subsection{Hand Magnetic Separation}

Do a hand separate to remove the really magnetic material (You don't want this material to clog the Franz while you are doing a magnetic separate.)

Magnet located in the flume room in the sed lab

Use two blue trays.

Need a .5L cup for the magnetic material

Need a small plastic funnel for transferring the sample back into a bag

Spread sample out over the tray

Run magnet through for 20 min or until satisfied with results (Have found that making a wedge of sample in a corner of the tray and then poking the magnet into the sample and letting it passively run over the magnet is the most efficient method)

Place magnetic material in the $.5 \mathrm{~L}$ cup (repeat until satisfied).

Place the newly separated material in two different bags.

Make sure to keep everything. 


\subsection{Frantzing}

For initial separation of mafic and felsic minerals (Old Frantz)

Two passes through the old frantz. (Keep the feeder mechanism in the back slot on the sample slide.)

First Pass at $\sim 20^{\circ}$ slope, with a .4 setting

This should split the really magnetic stuff out that hand separating did not.

Second Pass $2 \sim 5^{\circ}$ slope, with a 1.4 setting

This should be the main split from mafic to felsic material

\section{Separation for QTZ and Feldspar (New Frantz)}

Repeat this step as many times as necessary.

Move the feeder mechanism to the front slot on the sample slide.

Rotate the whole assemblage to -6 degree slope.

Readjust the feeder assemblage so that the upper portion (right side) is set at 0 and the lower portion (left side) of the sample slide is located at 5 (make sure it is the \#5 hash coming towards you [There are no + or - values on this section]). Pay attention to the ridge in the middle of the tray, the sample should just start to fall off when it hits the magnetic field. The feed rate will depend on sample (Rich had the feed set at 1.6 and the chute vibration set to 1.78) (Appendix Figure 1).

The current should be set at 1.8A (DON'T EVER TOUCH THE VOLTS KNOB!)

\section{Tips:}

The thinner the sample stream the cleaner the separate and the more quartz you will get out.

Use the dark bail to catch the material that you do not want (magnetic stuff and feldspar). Try to keep this consistent so that you don't dump a load of feldspar in with a fresh quartz separate.

Gently turn the current up and down do not rush it give the instrument a few seconds to warm up and cool down as you increase and decrease the current.

The left most adjustment knob on the bottom of the tray cradle is broken and may be difficult to work with (May have to tighten the knob head to remove/loosen the whole bolt).

If you need to clean up your sample (have too many fines) you can always resieve the sample at this point.

$\underline{10.5 .1} * *$ Cleaning the Frantz

\section{Be very gentle and careful with everything!}

\section{Disassembly:}

Remove the sample bails and empty sample into bag.

Remove the feeder pipe and disassemble for cleaning.

Detach the split pipes (New Frantz has a nut that you will need to undo)

Remove the hand bolts from the tray (should only ever be hand tight)

Very carefully remove the tray by sliding it out the long way, through the magnet, making sure not to lift and scratch the surface of the tray (\$20,000 to resurface!). 


\section{Vacuuming:}

Vacuum all the parts that have just come off of the Frantz.

Make sure to include the magnet while vacuuming.

Vacuum bench and surrounding area and the wipe everything down

\section{Ethanol:}

Spray all components with ethanol and wipe down with Kim Wipes (Make sure to spray into the corners and groves on the tray).

Run an ethanol-soaked Kim Wipe through the tray slot on the magnet.

\section{Compressed air:}

After the detachable parts have been cleaned with ethanol blow everything out with compressed air.

\section{Final:}

Lay all components upside down to protect the top surface from dust and scratches.

Set up for quartz-feldspar separation.

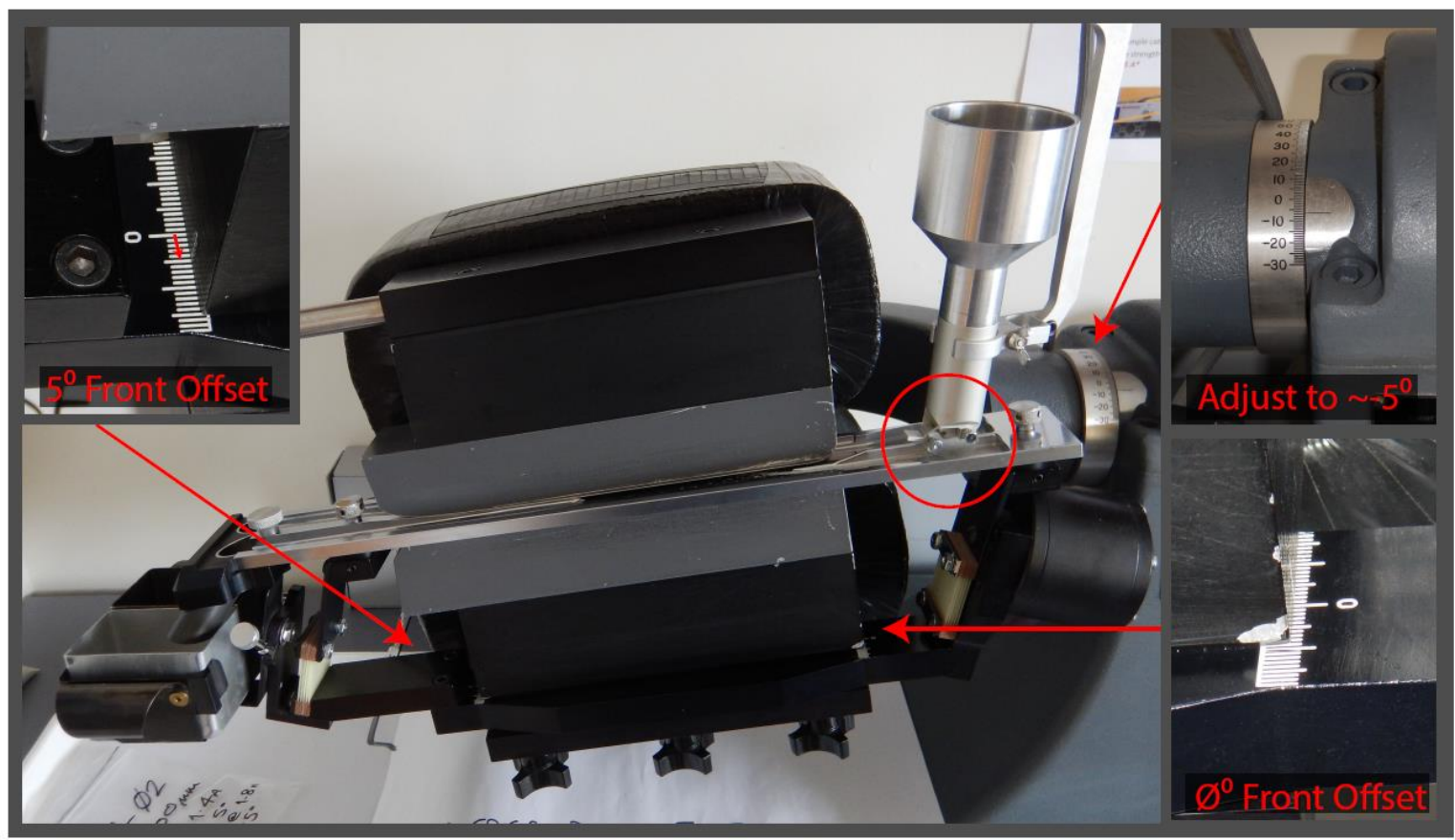

Appendix Figure 1: Diamagnetic Frantz Configuration

This is a Frantz magnetic mineral separator configured for diamagnetic (quartz-feldspar) mineral separation. The chute mount must be adjusted, the bottom (left) is adjusted $5^{0}$ and the top (right) remains centred. The main body of the magnet is placed in the rear chute slot. The feed tube is placed in the front chute slot (red circle). The whole assembly is rotated (back tilted) to approximately -5 . 


\section{Appendix B: Standard Chemical Processing Methods}

\section{QUARTZ PREPARATION AND SEPARATION OF COSMOGENIC BE AND AL}

Friedhelm v. Blanckenburg, Uni Hannover, Jan 2008

Modified by Kevin P. Norton, Uni Bern, May 2011

Modified by Richard S. Jones, VUW, June 2012

Modified by VUW CosmoLab group, April 2018

\subsection{Physical Quartz Separation}

If necessary, break samples into $\sim 5 \mathrm{~cm}$ chunks and crush down to $<1 \mathrm{~mm}$ using the jaw crusher.

Sieve dry sample to appropriate size fractions

Note: We typically use $\sim 0.25-0.5 \mathrm{~mm}$ fraction. However, smaller grain sizes down to $\sim 63$ um may be used when necessary.

If iron particle content is high: clean sample with hand magnet

If magnetic mineral content is high: Frantz magnetic separation

Note: You should aim to end the physical steps with > $200 \mathrm{~g}$ (dependant on Quartz content)

If feldspars are present: float feldspars

(see froth flotation method)

If persistent micas or other minerals remain: heavy liquid separation

\subsection{Chemical Quartz Cleaning and Etching}

You will need:

1L Nalgene bottles

$10 \% \mathrm{HCl}$ - (per 1L, $277 \mathrm{ml}$ conc. $(\sim 36 \%) \mathrm{HCl} ; \sim 723 \mathrm{ml}$ milliQ $\left.\mathrm{H}_{2} \mathrm{O}\right)$

$2.5 \% \mathrm{HF} / 1 \% \mathrm{HNO} 3$ - (per 1L, $\sim 50 \mathrm{ml}$ conc. $(\sim 48 \%) \mathrm{HF}: \sim 15 \mathrm{ml}$ conc. $(\sim 68 \%) \mathrm{HNO} 3$, $\sim 935 \mathrm{ml}$ milliQ H2O) Note: add water, then $\mathrm{HNO}_{3}$, then $\mathrm{HF}$ to sample

Notes: You must be wearing appropriate PPE when diluting acids. Always add acid to water, not the other way around. Scale acid volumes to size of mixing container.

Add $<100$ g of sample to a 1L Nalgene bottle. SLOWLY add $\sim 100 \mathrm{ml} 10 \% \mathrm{HCl}$ to the sample.

Notes: remember to add acid to water; If you have more than $100 \mathrm{~g}$ of sample, use additional $1 \mathrm{~L}$ bottles 
If there is a strong reaction (vigorous bubbling/foaming), leave sample uncapped overnight to react

If there is no visible reaction, fill bottle to $\sim 800 \mathrm{ml}$ with $10 \% \mathrm{HCl}$

Place on hotdog rollers for $\sim 1$ day $@ 60^{\circ} \mathrm{C}\left(\sim 50^{\circ} \mathrm{C}\right.$ on machine $)$

After 1 hour, retighten the lids on the bottles

Decant acid and wash with deionised/distilled water

Note: tip liquid of sample bottles into acid waste bucket, wash sample 3 times until water is clear

Repeat step if solution is very dirty (yellow)

Add $800 \mathrm{ml} 2.5 \% \mathrm{HF}$ to sample and spin on heated rollers or place in heated waterbath and agitate regularly for $\sim 1$ day

After 1 hour, retighten lids on the bottles

Note: Turn off rollers overnight

Decant acid and wash with deionized/distilled water

Note: wash at least $4 \mathrm{x}$, dumping into acid waste bucket; calcium carbonate should then be added to bucket (binding with fluoride) and soda ash (neutralising acid)

Add $800 \mathrm{ml} 2.5 \% \mathrm{HF}$ to sample and roll/heat for 2 days

Decant acid and wash with deionised/distilled water

Add $800 \mathrm{ml} 2.5 \% \mathrm{HF}$ to sample and roll/heat for 2 days

Decant acid and wash with deionised/distilled water

Note: this is the minimum treatment for removing meteoric ${ }^{10} \mathrm{Be}$ and impurities. A 'clean' sample will likely loose between $10-20 \%$ mass during these steps.

Repeat until sample is pure quartz (either visual inspection or $<5 \%$ weight change between steps)

Wash with $18.2 \Omega \mathrm{H}_{2} \mathrm{O}$ until $\mathrm{pH} \sim$ neutral, then dry at $60^{\circ} \mathrm{C}$ in the oven without lids and weigh sample

Note: You should aim to end the chemical cleaning steps with $>50 \mathrm{~g}$ of clean quartz. Very young/rapidly eroding samples will require more mass while very old/slowly eroding samples will require less mass. 


\title{
11.3 Chemical Cleaning
}

\author{
11.3.1 Final Quartz Leach
}

You will need:

$\mathrm{N}+1$ cleaned Savillex beakers (where $\mathrm{N}$ is your number of samples. See cleaning procedures at beginning of lab book)

Note: choose your beakers according to your dissolution method. For closed beaker dissolution (recommended), use $350 \mathrm{~mL}$ beakers for samples up to $45 \mathrm{~g}$ and $1000 \mathrm{~mL}$ beakers for anything larger. For open-beaker dissolution, you can use either $180 \mathrm{~mL}$ or 350 mL beakers.

7M HF - (per 1L, 250ml conc. (28M) HF; 750ml milliQ $\mathrm{H}_{2} \mathrm{O}$ ) Aqua Regia - (per 1L, 120ml conc. (15M) HNO3; 880ml conc. (6M) HCl)

Note: Aqua Regia is a 1:3 molar mixture of $\mathrm{HNO}_{3}: \mathrm{HCl}$. That means that these ratios will change if the either acid is at a different concentration. It must be made fresh for each use, do not leave Aqua Regia for more than a couple hours in any container.

Notes: You must be wearing appropriate PPE when diluting acids. Always add acid to water, not the other way around. Scale acid volumes to size of mixing container.

Take the precise weight of a 180 or $350 \mathrm{ml}$ Savillex screwtop jar and lid Note: this is one of three vital weights (along with final sample weight and carrier weight). Note: the large Savillex beakers must be weighed without lids. Add a maximum 50g quartz in $180 \mathrm{ml}$ jars and maximum $100 \mathrm{~g}$ in $350 \mathrm{ml}$ jars) for open-beaker dissolution. If using closed-beaker dissolution, use bigger jars - samples up to $45 \mathrm{~g}$ can go in the $350 \mathrm{~mL}$ jars. Note: if using the quick dissolution, only use the largest Savillex jars.

Note: label the beakers using a black permanent marker and write down the beaker number in your lab book.

Add sample to cleaned Savillex beakers

Add 7M HF till the sample is covered by $\sim 5 \mathrm{~mm}$ excess liquid

Cap and heat $\sim 1$ hour (maximum) @ $120^{\circ} \mathrm{C}$

Allow to cool, decant acid and wash $4 \mathrm{x}$ with $18.2 \Omega \mathrm{H}_{2} \mathrm{O}$

Note: large jars can be very difficult to open once cool. It is best to break the seal before they cool down.

Add Aqua Regia till the sample is covered by $\sim 5 \mathrm{~mm}$ excess liquid

Leave uncovered until all brown gas has gone, $\sim 30$ minutes

Cap and heat $\sim 2$ hours @ 120 C

Note: if leaving for a long period (i.e. overnight), tighten lid and leave off hotplate

Allow to cool, decant acid and wash 4-5x with $18.2 \Omega \mathrm{H}_{2} \mathrm{O}$

Dry samples without lids at $\sim 100^{\circ} \mathrm{C}$ on a hotplate

Allow to cool and take the precise weight (including Savillex jar and lid)

Note: this is the second vital weight 


\subsubsection{Carrier addition and sample dissolution}

You will need:

${ }^{9} \mathrm{Be}$ carrier solution - we use an in-house $305 \mathrm{ppm}{ }^{9} \mathrm{Be}$ solution calibrated by Shaun Eaves and Richard Jones, or a 1008.3 ppm solution from ANSTO (named ABAZ). Be sure you write down which carrier you use (and its concentration!) in your lab book.

Note: you are aiming for 0.2-0.3 mg 9Be depending on AMS facility. I.e., with a $305 \mathrm{ppm}$ carrier solution, then $0.8 \mathrm{~g}$ of carrier solution $=0.244 \mathrm{mg} 9 \mathrm{Be}$ )

Transfer enough carrier solution for your samples to a new, clean $15 \mathrm{ml}$ centrifuge tube. i.e. if you have 12 samples and need $\sim 0.8 \mathrm{ml}$ carrier per sample, then pour $9 \mathrm{ml}$ solution into the c-tube.

Take the precise weight of carrier added.

Note: this is the final vital weight...you can relax a bit now.

Note: we measure carrier by weight loss. Place the c-tube with carrier on the balance and zero. Use a $100-1000 \mu \mathrm{L}$ pipette, to transfer the required amount to the sample beaker, measuring mass loss from the carrier. Take precise weight and then re-zero before next sample.

This is a good time to start cleaing c-tubes for yourself (especially if you're doing Al chemistry). You'll need two (2) 50ml c-tubes per sample for Fe columns, four (4) 50ml c-tubes per sample for Be columns, and five (5) $15 \mathrm{ml} \mathrm{c-tubes} \mathrm{per} \mathrm{sample} \mathrm{for} \mathrm{sample} \mathrm{conversion} \mathrm{steps}$ (2) and precipitation (3). Finally, if you are doing Al chem you will need to clean one (1) $60 \mathrm{ml}$ Nalgene for per sample for your Al Aliquot.

Mix a $1 \% \mathrm{HCl}$ solution (there's a bottle for this purpose in the acid cabinet), fill your C-tubes just over halfway, and put them in the designated tupperware containers. Let them sit for a day, then flip the containers over and let them sit another day. Rinse x3 with DI water, then lay them out to dry in the windowsill with a clingwrap cover drapped over the top.

\section{$\underline{\text { 11.3.3 }}$ Bulk dissolution (closed beaker)}

You will need:

Conc. (28M) or $50 \%$ (this is what we get from suppliers) Trace metal grade HF concentrated HF is extremely dangerous. No lab user is to use concentrated HF without a safety buddy.

Note: You must be wearing all PPE when handling HF.

Conc. Trace metal grade $\mathrm{HNO}_{3}$.

Samples in Savillex (weighed!), with Be carrier added (lids on, so the carrier doesn't evaporate!) 
Calculate the required volume in $\mathrm{ml}$ of $\mathrm{HNO}_{3}(=1 \mathrm{ml} / \mathrm{g} \times$ quartz mass in grams $)$ and $\mathrm{HF}$ (= $5 \mathrm{ml} / \mathrm{g} \times$ quartz mass in grams) and record them in your lab notebook. When choosing volumes to be used for the process blank, use the average value for the batch.

Add $\mathrm{HF}$ to the sample beaker, then add $\mathrm{HNO}_{3}$.

Check your gloves for acid drops, and rinse or change gloves if needed.

Tighten lid, gently swirl the sample around, then loosen lid and place it in a safe place in the hood AWAY from the hotplate (no heat for the first 6 hours!). Let samples sit in the hood with loose lids for 6 hours, then tighten lids and move them onto the hotplate at $60^{\circ} \mathrm{C}$. Samples will dissolve faster if swirled several times a day to disrupt density stratification (swirl gently over the water bath or a sink, do not shake or invert beakers.)

Dissolution will take 2-3 days, but may take longer for larger samples. If you're planning to take an aliquot for $26 \mathrm{Al}$, do it now.

Fume off HF: move each beaker to hood, and carefully remove lids. Place beakers about a beaker-diameter apart from each other to avoid any contamination. Turn hotplate on to $120^{\circ} \mathrm{C}$, and evaporate HF. This will take a while (6-24 hours, depending on your acid volumes), and no other activities to happen in the hood with fuming samples - place a note on the hood to alert other lab users. If you have samples fuming overnight, turn down the hotplate to 100 , even for larger acid volumes.

Note: PPE is super important here. Never reach your hand or arm over an open beaker that's fuming!

Carefully remove from hotplate and allow to cool

Note: static electricity that can cause sample flakes to jump out of the beaker - use the antistatic gun to reduce static before touching the beakers. Wetting your gloves a bit and/or placing wet paper towels beneath the beakers can also help reduce this effect.

Note: at this stage you should have nothing more than a bit of white to grey/brown scum (or a thin white cake) in the bottom of your beaker. These are all the impurities that were in the quartz (Al, Fe, Mg, etc.). The following steps purify Be and/or Al.

\subsubsection{Dissolution version 1: slow and steady (open beaker)}

The stochiometric reaction for quartz dissolution is: $\mathrm{SiO}_{2}+4 \mathrm{HF}->\mathrm{SiF}_{4}+2 \mathrm{H}_{2} \mathrm{O}$ (e.g. you will need $116 \mathrm{ml} 28 \mathrm{M} \mathrm{HF}$ to dissolve $50 \mathrm{~g}$ quartz, however, in practice you will need a bit more)

You will need:

Conc. (28M) or $50 \% \mathrm{HF}$ - concentrated HF is extremely dangerous. No lab user is to use concentrated HF without a safety buddy.

Note: You must be wearing all PPE when handling HF. 
Add enough conc. HF to wet sample and wait a few minutes

If no reaction occurs continue adding conc. HF in increments till the sample is covered with $\sim 5 \mathrm{~mm}$ excess liquid

Note: the first couple HF additions may generate a strong exothermic reaction, so be careful

Allow to react unheated for $\sim 15$ minutes

Heat on hotplate without lid @ $120^{\circ} \mathrm{C}$, eventually evaporating to dryness

Note: keep samples at least one beaker width apart as samples may splatter if the reaction becomes strong.

Repeat HF addition and dry-down until all quartz is dissolved

Note: after the $2^{\text {nd }}$ or $3^{\text {rd }} \mathrm{HF}$ addition, the reaction should be less volatile, and you can add HF up to twice the quartz volume.

You can cap the sample and create pressure vessile which changes the partial pressure of the system and helps drive the digestion reaction, though not for long periods of time (e.g., you can leave it over night at 60C and uncap the sample in the morning.)

You can also use closed beaker dissolution as above until your sample volume is sufficiently reduced, and then move to closed beaker dissolution.

Carefully remove from hotplate and allow to cool

Note: static electricity that can cause sample flakes to jump out of the beaker - use the antistatic gun to reduce static before touching the beakers. Wetting your gloves a bit and/or placing wet paper towels beneath the beakers can also help reduce this effect.

Note: at this stage you should have nothing more than a bit of white to grey/brown scum (or a thin white cake) in the bottom of your beaker. These are all the impurities that were in the quartz ( $\mathrm{Al}, \mathrm{Fe}, \mathrm{Mg}$, etc.). The following steps purify Be and/or Al. 


\subsection{Chemical Separation}

\subsubsection{Sample conversion and Al-chemistry}

\section{(To be done if Al will/might be measured)}

You will need:

Aqua Regia - (per 1L, 120ml conc. (15M) HNO3; 880ml conc. (6M) HCl)

Note: Aqua Regia is a 1:3 molar mixture of $\mathrm{HNO}_{3}: \mathrm{HCl}$. That means that these ratios will change if the either acid is at a different concentration. It must be made fresh for each use, do not leave Aqua Regia for more than a couple hours in any container.

For 12 samples at $20 \mathrm{ml} / \mathrm{sample}$, this is: $30 \mathrm{ml} 15 \mathrm{M} \mathrm{HNO}, \sim 210 \mathrm{ml} 6 \mathrm{M} \mathrm{HCl}$

$6 \mathrm{M} \mathrm{HCl}-$ our clean $\mathrm{HCl}$ is provided at $6 \mathrm{M}$ by Louisa

$3 \mathrm{M}$ HNO3 - only if doing $\mathrm{Al}$ chemistry (per 1L, 200ml conc. (15M) HNO3, 800ml 18.2

$\Omega \mathrm{H} 2 \mathrm{O})$.

Notes: You must be wearing appropriate PPE when diluting acids. Always add acid to water, not the other way around. Scale acid volumes to size of mixing container.

$\mathrm{Nx} 215 \mathrm{ml}$ c-tubes

$\mathrm{N} 60 \mathrm{ml}$ bottles

Note: always use new, cleaned c-tubes and bottles. See cleaning procedures in lab manual.

Add 20ml Aqua Regia to each sample

Cap the beakers and heat at $60^{\circ} \mathrm{C}$ until all the fluoride residue is redissolved

Note: this might be instantaneous or take a few hours.

Heat at $90^{\circ} \mathrm{C}$ to evaporate gently

Note: takes $\sim 1 / 2$ day - do not burn sample!

Add $10 \mathrm{ml} 6 \mathrm{M} \mathrm{HCl}$ to each sample to redissolve the residue

Once dissolved, transfer sample into new, cleaned $15 \mathrm{ml}$ (or $50 \mathrm{ml}$ for dirty samples that require more $\mathrm{HCl}$ ) centrifuge tubes

Centrifuge for 5 minutes @ 3000 rpm

Note: if only doing Be chemistry, stop here and proceed to step 5.

Place a new, clean c-tube on balance, zero balance

Transfer sample solution into centrifuge tube, leave undissolved residue behind

Take precise weight of TSS (total sample solution).

Note: this is the first of four vital weights for Al.

Precisely weigh a new, clean $60 \mathrm{ml}$ bottle.

Note: this is the second vital weight for Al.

Transfer a $250 \mu 1$ aliquot of your TSS into the $60 \mathrm{ml}$ bottle

Take precise weight of $\mathrm{Al}$ aliquot.

Note: this is the third vital weight for Al.

Add $5 \mathrm{ml} 3 \mathrm{M} \mathrm{HNO}_{3}$ to $\mathrm{Al}$ aliquot (storage in strong acid to prevent adsorption of $\mathrm{Al}$ )

Take precise weight of the bottle.

Note: this is the final vital weight for $\mathrm{Al}$.

Before ICP-MS measurement: dilute to $1 \% \mathrm{M} \mathrm{HNO}_{3}$ by addition of $62 \mathrm{ml} 18.2 \Omega \mathrm{H}_{2} \mathrm{O}$ 
11.4.2 BeF2 - Leaching (only when NO Al-chemistry will be done)

$\mathrm{BeF}_{2}$ is water soluble while many other cations (including Al) are less so.

You will need:

$18.2 \Omega \mathrm{H}_{2} \mathrm{O}$ - this is from the Elga water purifier.

$\mathrm{N} 22 \mathrm{ml}$ or $180 \mathrm{ml}$ Savillex beakers

$\mathrm{N} 50 \mathrm{~mL}$ c-tubes (for dirty samples) or $15 \mathrm{~mL}$ tubes (for clean samples)

Add $10 \mathrm{ml} 18.2 \Omega \mathrm{H}_{2} \mathrm{O}$ to fluoride cake (to cover bottom of beaker)

Note: for clean samples you will need $5 \mathrm{ml} 18.2 \Omega \mathrm{H}_{2} \mathrm{O}$

Let sit for $\sim 20$ minutes at $50^{\circ} \mathrm{C}$, then pour off into a c-tube. Repeat with $5 \mathrm{~mL}$ MQ water (this

serves as a rinse). Move any residue in the savillex into the c-tubes, and wipe out savillex with a kimwipe.

Vortex c-tube for 1 minute and then let rest for 10 minutes at room temperature.

Centrifuge for $5 \mathrm{~min}$ at 3000rpm

Pour off the supernate (containing water-soluble $\mathrm{BeF}_{2}, \mathrm{TiF}_{4}, \mathrm{Fe}(\mathrm{II}) \mathrm{F}_{2}$ but no $\mathrm{AlF}_{3}$ ) into clean Savillex beakers.

Add $15 \mathrm{~mL}$ MQ water to the c-tube. Vortex, let sit 10 minutes.

Centrifuge, then pour supernate into the savillex.

Repeat once more: add $15 \mathrm{~mL}$ water to c-tube, vortex, centrifuge, pour off supernate into savillex.

Heat the samples in savillex without lids at $\sim 120^{\circ} \mathrm{C}$ to evaporate the solution

Note: keep beakers at least one beaker diameter apart to reduce the risk of crosscontamiation.

Add $10 \mathrm{ml} 6 \mathrm{M} \mathrm{HCl}$ to each sample

Once fully dissolved, transfer solution into cleaned $15 \mathrm{ml}$ centrifuge tubes

Centrifuge for 5 minutes @ 3000 rpm

\section{$\underline{11.4 .3}$ Column Fe}

$2 \mathrm{ml}$ Biorad AG1-X8 100-200 mesh (anion) resin in 15ml Eichrom columns stored in $\mathrm{H}_{2} \mathrm{O}$. Note: to prepare new columns 1) place empty columns in stand with shot glasses below, 2) add tip to one column, add $2 \mathrm{ml}$ of $\mathrm{H}_{2} \mathrm{O}$ and mark line (then mark line on other columns), 3) add water and resin to a small beaker and agitate, 4) wet filter for each column and check they are dripping, 5) slowly pipette resin into each column, letting it settle, and continue until resin reaches the marked line, 6) to store, fill with $\mathrm{H}_{2} \mathrm{O}$, cap tops and add tips to ends.

DON'T LET THE RESINS DRY OUT.

You will need:

$0.3 \mathrm{M} \mathrm{HCl}$ - (for $1 \mathrm{~L}$ add $20 \mathrm{ml}$ conc. (15M) HNO3 to $980 \mathrm{ml} 18.2 \Omega \mathrm{H} 2 \mathrm{O}$ ). $6 \mathrm{M} \mathrm{HCl}$ 
0.4M Oxalic acid - (for $2 \mathrm{~L}$ add $101.2 \mathrm{~g}$ solid oxalate (measured on tared filter paper) to $2 \mathrm{~L}$ reagent bottle, then add $2 \mathrm{~L} 18.2 \Omega \mathrm{H} 2 \mathrm{O}$, shake to dissolve (this usually takes an hour or two). (note, the bottle is actually a bit small, mix up 1.8L at a time - be precise! (do the math) $\mathrm{N} 2 \mathrm{ml}$ anion columns

N Savillex beakers (note, these can be your cleaned digestion beakers - rinse with MQ water and wipe them out with a kimwipe)

$\mathrm{N}$ waste containers (or $15 \mathrm{ml}$ c-tubes if you will be keeping wash solution)

$\mathrm{N} 15 \mathrm{ml}$ c-tubes

Column rack

Open column and let the water drip out

Add $5 \mathrm{ml}+5 \mathrm{ml} 0.3 \mathrm{M} \mathrm{HCl}$ to clean the resin

Add $2 \mathrm{ml}+2 \mathrm{ml}+2 \mathrm{ml} 6 \mathrm{M} \mathrm{HCl}$ to condition the resin (drain to waste container) (drain to waste container) (drain to waste container)

Add sample $(10 \mathrm{ml} 6 \mathrm{M} \mathrm{HCl}) \quad$ Collect Be $(+\mathbf{A l})$ in Savillex beakers

Note: either pipette or slowly decant the solution into the columns. Be careful not to disturb the resin and do not pour any solids onto the resin.

Add $2 \mathrm{ml}+2 \mathrm{ml}+2 \mathrm{ml} 6 \mathrm{M} \mathrm{HCl}$ to elute Be Collect Be $(+\mathbf{A l})$ in Savillex beakers

Add $5 \mathrm{ml}+5 \mathrm{ml} 0.3 \mathrm{M} \mathrm{HCl}$ to clean the resin (drain to waste container)

Add $18.2 \Omega \mathrm{H}_{2} \mathrm{O}$ and seal the columns for storage

Heat the samples without lids at $\sim 120^{\circ} \mathrm{C}$ till dry

Add $8 \mathrm{ml}$ (20ml for dirty quartz) $0.4 \mathrm{M}$ oxalic acid to each sample

Warm the samples at $60^{\circ} \mathrm{C}$ with lid for $\sim 2$ hours

Note: Oxalic acid decomposes at high temperatures. Be cautious.

Remove from hotplate and allow to cool down for at least 30 minutes

Transfer the samples to new, clean $15 \mathrm{ml}$ centrifuge tubes

Centrifuge for 5 minutes@ 3000 rpm

\section{$\underline{11.4 .4} 5 \mathrm{ml}$ Column Be (Dirty Samples: $\mathrm{Al}>5 \mathrm{mg}$ )}

$5 \mathrm{ml}$ Biorad AG50-X8 200-400 mesh (cation) resin in $15 \mathrm{ml}$ Eichrom columns stored in $\mathrm{H}_{2} \mathrm{O}$. Note: This step takes the better part of a day. Make sure you have enough acid mixed before you start to avoid having to stop and mix acid during columns. Check mixed acid densities, and be aware that you may need to wait a bit for more concentrated acids to cool before you test them (hot=less dense!).

Open column and let the water drip out Add $5 \mathrm{ml}+10 \mathrm{ml} \sim 5 \mathrm{M} \mathrm{HNO}_{3}$ to clean the resin (drain to waste container) (drain to waste container)

Add $5 \mathrm{ml}+5 \mathrm{ml} 18.2 \Omega \mathrm{H}_{2} \mathrm{O}$ to remove $\mathrm{HNO}_{3}$ from the resin (drain to waste container) Note: you can prep columns to this point the day before if needed; fill with MQ water and cap, then let drain to waste container before proceeding.

Add $5 \mathrm{ml}+10 \mathrm{ml} 0.4 \mathrm{M}$ oxalic acid to condition resin Add sample (20ml 0.4M oxalic acid) 
Add 5ml 0.4M oxalic acid to wash the sample down

(drain to c-tube)

Add $5 \mathrm{ml} 0.4 \mathrm{M}$ oxalic acid to wash the sample down (drain to c-tube) Add $50 \mathrm{ml} 0.4 \mathrm{M}$ oxalic acid: to elute $\mathrm{Fe}, \mathrm{Al}$, Ti etc. (drain to c-tube) Add $5 \mathrm{ml}+10 \mathrm{ml} 18.2 \Omega \mathrm{H}_{2} \mathrm{O}$ to remove oxalic acid (drain to c-tube)

Note: sample in $\mathrm{H}_{2} \mathrm{O}$ and oxalic acid will need to be collected in 2x clean 50ml centrifuge tubes per column

Add $15 \mathrm{ml}+25 \mathrm{ml} 0.5 \mathrm{M} \mathrm{HNO}_{3}$ to elute $\mathrm{Na}$ (drain to clean c-tube)

Note: Na will need to be collected in $1 \mathrm{x}$ clean $50 \mathrm{ml}$ centrifuge tube per column Add $20 \mathrm{ml} 1 \mathrm{M} \mathrm{HNO}_{3}$ wash (drain to savillex)

Note: you can use the same savillex beakers from previous steps - just be sure they've been rinsed/wiped out!

Add $20 \mathrm{ml}+20 \mathrm{ml} 1 \mathrm{M} \mathrm{HNO}_{3}$ to elute Be

Collect Be in 90ml Savillex beakers

Add $40 \mathrm{ml} 5 \mathrm{M} \mathrm{HNO}_{3}$ to clean resin (drain to clean c-tubes)

Note: post-Be wash will need to be collected in $1 \mathrm{x}$ clean $50 \mathrm{ml}$ centrifuge tubes per column Add $5 \mathrm{ml}+15 \mathrm{ml} \mathrm{H}_{2} \mathrm{O}$ remove $5 \mathrm{M} \mathrm{HNO}_{3}$ (drain to waste container)

Add $18.2 \Omega \mathrm{H}_{2} \mathrm{O}$ and seal the columns for storage

Heat the samples in savillex at $\sim 120^{\circ} \mathrm{C}$ to evaporate the solution Add $5 \mathrm{ml} 1 \mathrm{M} \mathrm{HNO}_{3}$ to dissolve the sample and transfer to a new $15 \mathrm{ml}$ centrifuge tube

\section{$\underline{11.4 .5}$ Column Al}

Iml Biorad AG1-X8 100-200 mesh (anion) resin in 7.5ml columns stored in $\mathrm{H}_{2} \mathrm{O}$ Note: to prepare mixture of $0.05 \mathrm{M}$ Oxalic Acid and $0.5 \mathrm{M} \mathrm{HCl}$, mix equal amounts of $0.1 \mathrm{M}$ oxalic Acid and $1 \mathrm{M} \mathrm{HCl}$.

Open column and let $\mathrm{H}_{2} \mathrm{O}$ drop out Add $2 \mathrm{ml} 18.2 \Omega \mathrm{H}_{2} \mathrm{O}$ to clean resin Add $5 \mathrm{ml}+5 \mathrm{ml} 0.3 \mathrm{M} \mathrm{HCl}$ to clean resin Add $2 \mathrm{ml}+2 \mathrm{ml} 18.2 \Omega \mathrm{H}_{2} \mathrm{O}$ to remove $\mathrm{HCl}$ Add $2 \mathrm{ml}+2 \mathrm{ml}+2 \mathrm{ml} 0.4 \mathrm{M}$ oxalic Acid to condition resin

Add sample (0.4M oxalic acid from Be-column) Add $4 \mathrm{ml}+2 \mathrm{ml} 0.05 \mathrm{M} \mathrm{Ox} / 0.5 \mathrm{M} \mathrm{HCl}$ wash Note: pre-Al effluent will need to be collected in $1 \mathrm{x}$ cleaned $50 \mathrm{ml}$ centrifuge tube per column (in case $\mathrm{Al}$ elutes early)

Add $5 \mathrm{ml}+5 \mathrm{ml} 0.05 \mathrm{M} \mathrm{Ox} / 0.5 \mathrm{M} \mathrm{HCl}$ beakers

Add $5 \mathrm{ml}+5 \mathrm{ml} 0.3 \mathrm{M} \mathrm{HCl}$ to elute Ti

Note: Ti will need to be collected in $1 \mathrm{x}$ cleaned $11 \mathrm{ml}$ centrifuge tube per column Note: to make up $0.3 \mathrm{M} \mathrm{HCl}$ (for $140 \mathrm{ml}), 136 \mathrm{ml} \mathrm{H}_{2} \mathrm{O}+4 \mathrm{ml}(\sim 10.5 \mathrm{M}) \mathrm{HCl}$.

Seal and store column in $18.2 \Omega \mathrm{H}_{2} \mathrm{O}$

Dry down sample in Savillex beaker on hotplate (over $1 / 2$ day or overnight) Add $2 \mathrm{ml}$ Aqua Regia (15M HNO $1: 16 \mathrm{M} \mathrm{HCl}$ ) and leave on hotplate to dissolve with lid 
Note: to make up $\sim 30 \mathrm{ml}$, add $15 \mathrm{ml} \mathrm{HNO}_{3}(15 \mathrm{M})+\sim 8.6 \mathrm{ml} \mathrm{HCl}(\sim 10.5 \mathrm{M})+6.4 \mathrm{ml} \mathrm{H}_{2} \mathrm{O}$

Remove lid and evaporate on hotplate to dryness

Repeat once

Add $1 \mathrm{ml} 15 \mathrm{M} \mathrm{HNO}_{3}$ and $1 \mathrm{ml} \mathrm{H}_{2} \mathrm{O}_{2}$

Evaporate on hotplate to dryness

Repeat 2x

Go to Al precipitation or silver addition

\section{$\underline{\text { 11.4.6 }}$ Be precipitation}

Add $0.550 \mathrm{ml}$ concentrated $(\sim 25 \%) \mathrm{NH}_{4} \mathrm{OH}$ to each sample (will be $\mathrm{pH} \approx 10$ )

Shake the sample well (or vortex) until a $\mathrm{BeOH}$ precipitate forms

Note: small white specks will appear in the solution; let it sit at least 15 minutes

Centrifuge for 5 minutes @ 3000 rpm

Note: you should see a small milky gel at the bottom of the tube, this is $\mathrm{BeOH}$ !

Decant the supernate into the $5 \mathrm{M} \mathrm{HNO}_{3}$ centrifuge tubes with post-Be wash

Redissolve the precipitate in $5 \mathrm{ml} 1 \mathrm{M} \mathrm{HNO}_{3}$

Transfer to new $11 \mathrm{ml}$ or $15 \mathrm{ml}$ centrifuge tubes

Add $0.550 \mathrm{ml}$ concentrated $\mathrm{NH}_{4} \mathrm{OH}$ to each sample

Centrifuge for 5 minutes @ 3000 rpm

Decant the supernate

Add $3 \mathrm{ml} \mathrm{H}_{2} \mathrm{O}$ and shake well to dissociate the precipitate

Centrifuge for 5 minutes @ 3000 rpm

Decant the supernate

\section{$\underline{11.4 .7} \mathrm{Al}$ precipitation}

Add 5ml 1M $\mathrm{HNO}_{3}$ to samples (in Savillex beaker) and dissolve on hotplate

Transfer samples to cleaned $15 \mathrm{ml}$ centrifuge tube

Add $\sim 475 \mu \mathrm{L}$ conc. $\mathrm{NH}_{4} \mathrm{OH}$ to each sample $-\mathrm{pH}$ will be $7-8$

Shake the sample well until a precipitate forms

Note: white specks will appear in the solution (more obvious than with Be)

Centrifuge for 5 minutes @ $3000 \mathrm{rpm}$

Decant the supernate

Add $\mathrm{H}_{2} \mathrm{O}$ and shake well to dissociate the precipitate

Centrifuge for 5 minutes @ 3000 rpm

Decant the supernate

Add $\mathrm{H}_{2} \mathrm{O}$ and shake well to dissociate the precipitate

Centrifuge for 5 minutes @ 3000 rpm 


\section{$\underline{11.4 .8}$ Be Oxide + Silver}

The silver addition is for ETH-AMS only, many other labs use only BeO mixed with $\mathrm{Nb}$. Mix the silver solution: $157 \mathrm{mg} \mathrm{AgNO}_{3}$ into $10 \mathrm{ml} 5 \mathrm{M} \mathrm{HNO}_{3}$

Add $0.3 \mathrm{ml} \mathrm{Ag} \mathrm{solution} \mathrm{to} \mathrm{the} \mathrm{precipitate} \mathrm{(add} \mathrm{proportionally} \mathrm{less} \mathrm{for} \mathrm{samples} \mathrm{where} \mathrm{some}$ ${ }^{9}$ Be was lost during chemistry)

(Aim: $\mathrm{Ag}: \mathrm{Be}=20: 1$ => 4.7mg AgNO3 for $0.15 \mathrm{mg}$ Carrier)

Transfer the solution to a new clean quartz crucible

Dry the samples beneath red lamps or on hotplate in ceramic holder $\sim 150^{\circ} \mathrm{C}$

Oxidize in the samples in an oven or over a Bunsen burner

Using Pt-coated tongs, dry the sample for $\sim 60$ seconds towards the outside of the flame, then $\sim 1$ minute in blue part of flame to oxidize (the sample should be glowing).

Use a metal spatula to scratch the sample from the walls of the crucible

\section{$\underline{11.4 .9}$ Targets}

\section{For ETH-AMS targets}

Work with dust mask or in fume hood!

Be is very dangerous when inhaled

Clean the copper targets for 1 minute in $1 \mathrm{M} \mathrm{HCl}$ and rinse with $\mathrm{H}_{2} \mathrm{O}$

Dry the clean targets under a red lamp or on a hotplate

Clean all instruments with ethanol

Scratch the sample from the crucible walls with a metal spatula

Carefully transfer the sample from the crucible into the rear of the target with the spatula (use cleaned steel plate coated with $\mathrm{Al}$ foil for a base)

Gently hammer the sample into the target (repeated light blows)

Once all the sample has been added, add $63 \mu \mathrm{m}$ copper powder (p.a., 2703) until the target is filled

Label the target with the sample number on the front face using an Edding marker.

\section{For PRIMELab and LLNL targets}

Work with dust mask or in fume hood!

Be is very dangerous when inhaled

Clean the stainless steel targets one at a time and do it quickly in $1 \mathrm{M} \mathrm{HCl}$ and rinse with $\mathrm{H}_{2} \mathrm{O}$ (The $\mathrm{HCl}$ will etch the stainless if you are not quick enough)

Dry the clean targets with a kimwipe ensuring that there is no fluid left in the target pit.

Clean all instruments with ethanol

Scratch the sample from the crucible walls with a metal spatula or drill blank. 
Add $\sim 3$ micrograms $\mathrm{Nb}$ powder (4 level scoops) to the sample in the crucible and mix $\mathrm{BeO}$ and $\mathrm{Nb}$ together.

Optional: Pack a small amount of $\mathrm{Cu}$ or $\mathrm{Nb}$ powder into the target to raise the level that your sample will sit at while being run. (gently hammer $\mathrm{Cu}$ base into the target: LIGHT BLOWS!)

Clean the drill blank after use with $\mathrm{Cu}$ powder

Carefully transfer the sample from the crucible into the top of the target with the spatula (use cleaned steel plate and top assembly with Al foil or filterpaper for a base)

Gently hammer the sample into the target (repeated light blows)

Put completed target into plastic shipping holder and stuff with part of a kimwipe to stabilize the target while in transit.

Cap and Label the shipping holder with the sample number and target number (assigned by the lab) on the front face using an Edding marker, and note the sample number and the target number in your lab book.

Clean all instruments with ethanol

\section{Adapted by:}

With help from: Mirjam Schaller, Veerle Vanacker, Hella Wittmann, Kevin Norton, Jérôme Chmeleff; and suggestions by Darryl Granger

Updated by the VUW lab team in 2018, with help from Ross Whitmore, Jamey Stutz, and Claire Lukens. 


\section{REFERENCES}

Ackert, R.P., Barclay, D.J., Borns, H.W., Calkin, P.E., Kurtz, M.D., Fastook, J.L., and Steig, E.J., 1999, Ackert_99_ER-WA: Science, v. 286, p. 276-280.

Anderson, C.D., 1932, Energies of cosmic-ray particles: Physical Review, v. 41, p. 405-421, doi:10.1103/PhysRev.41.405.

Anderson, J.B. et al., 2014, Ross Sea paleo-ice sheet drainage and deglacial history during and since the LGM: Quaternary Science Reviews, doi:10.1016/j.quascirev.2013.08.020.

Anderson, J.T.H., Wilson, G.S., Fink, D., Lilly, K., Levy, R.H., and Townsend, D., 2017, Reconciling marine and terrestrial evidence for post LGM ice sheet retreat in southern McMurdo Sound, Antarctica: Quaternary Science Reviews, v. 157, p. 1-13, doi:10.1016/j.quascirev.2016.12.007.

Arcy, M.D., Roda-boluda, D.C., and Whittaker, A.C., 2017, Glacial-interglacial climate changes recorded by debris fl ow fan: Quaternary Science Reviews, v. 169, p. 288-311, doi:10.1016/j.quascirev.2017.06.002.

Arthern, R.J., and Williams, C.R., 2017, The sensitivity of West Antarctica to the submarine melting feedback: Geophysical Research Letters, v. 44, p. 2352-2359, doi:10.1002/2017GL072514.

Assmann, K.M., Darelius, E., Wåhlin, A.K., Kim, T.W., and Lee, S.H., 2019, Warm Circumpolar Deep Water at the Western Getz Ice Shelf Front, Antarctica: Geophysical Research Letters, v. 46, p. 870-878, doi:10.1029/2018GL081354.

Augustinus, P.C., 2002, Weathering Characteristics of the Glacial Drifts, Bunger Hills, East Antarctica: Arctic, Antarctic, and Alpine Research, v. 34, p. 65-75, doi:10.1080/15230430.2002.12003470.

Balco, G., 2011, Contributions and unrealized potential contributions of cosmogenic-nuclide exposure dating to glacier chronology, 1990-2010: Quaternary Science Reviews, v. 30, p. 3-27, doi:10.1016/j.quascirev.2010.11.003.

Balco, G., and Shuster, D.L., 2009, Production rate of cosmogenic 21Ne in quartz estimated from 10Be, 26Al, and $21 \mathrm{Ne}$ concentrations in slowly eroding Antarctic bedrock surfaces: Earth and Planetary Science Letters, v. 281, p. 48-58, doi:10.1016/j.epsl.2009.02.006.

Balco, G., Stone, J.O., Lifton, N.A., and Dunai, T.J., 2008, A complete and easily accessible means of calculating surface exposure ages or erosion rates from 10Be and 26Al measurements: Quaternary Geochronology, v. 3, p. 174-195, doi:10.1016/j.quageo.2007.12.001.

Balco, G., Todd, C., Goehring, B.M., Moening-Swanson, I., and Nichols, K., 2019, Glacial geology and cosmogenic-nuclide exposure ages from the Tucker glacier - Whitehall glacier confluence, northern Victoria Land, Antarctica: American Journal of Science, v. 319, p. 255-286, doi:10.2475/04.2019.01.

Bamber, J.L., Layberry, R.L., and Gogineni, S.P., 2001, A new ice thickness and bed data set for the Greenland ice sheet: 1. Measurement, data reduction, and errors: Journal of Geophysical Research Atmospheres, v. 106, p. 33773-33780, doi:10.1029/2001JD900054.

Bamber, J.L., Vaughan, D.G., and Joughin, I., 2000, Widespread complex flow in the interior of the antarctic ice sheet: Science, v. 287, p. 1248-1250, doi:10.1126/science.287.5456.1248.

Banwell, A.F., Macayeal, D.R., and Sergienko, O. V, 2013, Breakup of the Larsen B Ice Shelf triggered by chain reaction drainage of supraglacial lakes: Geophysical Research Letters, v. 40, p. 5872-5876, doi:10.1002/2013GL057694. 
Bard, E., Hamelin, B., Arnold, M., Montaggioni, L., Cabioch, G., Faure, G., and Rougerie, F., 1996, Bard_96_MWP-1a: Nature, v. 382, p. 241-244.

Baroni, C., and Hall, B.L., 2004, A new Holocene relative sea-level curve for Terra Nova Bay, Victoria Land, Antarctica: Journal of Quaternary Science, doi:10.1002/jqs.825.

Barry, R.G., and Gan, T.Y., 2011, The Global Cryosphere:, doi:10.1017/CB09780511977947.

Beer, J., McCracken, K., and Steiger, R. von, 2012, Cosmogenic Radionuclides: 426 p.

Bennett, M.R., 2003, Ice streams as the arteries of an ice sheet : their mechanics, stability and significance: Earth-Science Reviews, v. 61, p. 309-339, doi:10.1016/S0012-8252(02)00130-7.

Benson, L., Madole, R., Phillips, W., Landis, G., Thomas, T., and Kubik, P., 2004, The probable importance of snow and sediment shielding on cosmogenic ages of north-central Colorado Pinedale and pre-Pinedale moraines: Quaternary Science Reviews, doi:10.1016/j.quascirev.2003.07.002.

Berner, R.A., 1992, Weathering, plants, and the long-term carbon cycle: Geochimica et Cosmochimica Acta, v. 56, p. 3225-3231, doi:10.1016/0016-7037(92)90300-8.

Berner, R.A., Lasaga, A.C., and Garrels, R.M., 1983, Berner_83_Mg-Ca-model: American Journal of Science, v. 283, p. 641-683.

von Blanckenburg, F., 2005, The control mechanisms of erosion and weathering at basin scale from cosmogenic nuclides in river sediment: Earth and Planetary Science Letters, doi:10.1016/j.epsl.2005.06.030.

Von Blanckenburg, F., Belshaw, N.S., and O'nions, R.K., 1996, Separation of 9Be and cosmogenic $1^{\circ}$ Be from environmental materials and SIMS isotope dilution analysis.:

Blard, P.H., Bourlès, D., Pik, R., and Lavé, J., 2008, In situ cosmogenic 10Be in olivines and pyroxenes: Quaternary Geochronology, v. 3, p. 196-205, doi:10.1016/j.quageo.2007.11.006.

Blatt, H., and Jones, R.L., 1975, Proportions of exposed igneous, metamorphic, and sedimentary rocks: Bulletin of the Geological Society of America, v. 86, p. 1085-1088, doi:10.1130/00167606(1975)86<1085:POEIMA>2.0.CO;2.

Branch, I., Goddard, N., and Flight, S., 1983, THE IMPORTANCE OF PRESSURIZED SUBGLACIAL WATER IN: Journal of Glaciology, v. 29.

Braucher, R., Benedetti, L., Bourlès, D.L., Brown, E.T., and Chardon, D., 2005, Use of in situ-produced 10Be in carbonate-rich environments: A first attempt: Geochimica et Cosmochimica Acta, v. 69, p. 14731478, doi:10.1016/j.gca.2004.09.010.

Broeke, M.R. Van Den, and Bamber, J., 2011, Ice Sheets and Sea Level : Thinking Outside the Box: Survay of Geophysics, p. 495-505, doi:10.1007/s10712-011-9137-z.

Bromley, G.R.M., Hall, B.L., Stone, J.O., Conway, H., and Todd, C.E., 2010, Late Cenozoic deposits at Reedy Glacier, Transantarctic Mountains: implications for former thickness of the West Antarctic Ice Sheet: Quaternary Science Reviews, doi:10.1016/j.quascirev.2009.07.001.

Brook, E.J., Brown, E.T., Kurz, M.D., Ackert, R.P., Raisbeck, G.M., and Yiou, F., 1995, Constraints on age, erosion, and uplift of Neogene glacial deposits in the Transantarctic Mountains determined from in situ cosmogenic 10 Be and 26 Al: Geology, v. 23, p. 1063-1066, doi:10.1130/00917613(1995)023<1063:COAEAU>2.3.CO;2. 
Brook, E.J., Kurz, M.D., and Ackert, R.P., 193AD, Chronology-of-Taylor-Glacier-Advances-in-Arena-Valley-Antarctica--Using-in-Situ-Cosmogenic-3He-and-10Be_1993_Quaternary-Research.pdf: Quarternary Research, v. 39, p. 11-23.

Brown, E.T., Edmond, J.M., Raisbeck, G.M., Yiou, F., Kurz, M.D., and Brook, E.J., 1991, Examination of surface exposure ages of Antarctic moraines using in situ produced 10Be and 26Al: Geochimica et Cosmochimica Acta, v. 55, p. 2269-2283, doi:10.1016/0016-7037(91)90103-C.

Bruno, L.A., Baur, H., Graf, T., Schlüchter, C., Signer, P., and Wieler, R., 1997, Dating of Sirius Group tillites in the Antarctic Dry Valleys with cosmogenic 3He and 21Ne: Earth and Planetary Science Letters, v. 147, p. 37-54, doi:10.1016/s0012-821x(97)00003-4.

Carracedo, A., Rodés, Smellie, J.L., and Stuart, F.M., 2019, Episodic erosion in West Antarctica inferred from cosmogenic $3 \mathrm{He}$ and 10Be in olivine from Mount Hampton: Geomorphology, v. 327, p. 438-445, doi:10.1016/j.geomorph.2018.11.019.

Chmeleff, J., von Blanckenburg, F., Kossert, K., and Jakob, D., 2010, Determination of the 10 Be half-life by multicollector ICP-MS and liquid scintillation counting: Nuclear Instruments and Methods in Physics Research, Section B: Beam Interactions with Materials and Atoms, v. 268, p. 192-199, doi:10.1016/j.nimb.2009.09.012.

Church, J.A., P.U. Clark, A. Cazenave, J.M. Gregory, S. Jevrejeva, A. Levermann, M.A. Merrifield, G.A. Milne, R.S. Nerem, P.D. Nunn, A.J. Payne, W.T. Pfeffer, D.S. and A.S.U., 2013, Sea Level Change: Sea Level Change. In: Climate Change 2013: The Physical Science Basis. Contribution of Working Group I to the Fifth Assessment Report of the Intergovernmental Panel on Climate Change, p. 1137-1177.

Clark, P.U., Mitrovica, J.X., Milne, G. a, and Tamisiea, M.E., 2002, Sea-level fingerprinting as a direct test for the source of global meltwater pulse IA.: Science (New York, N.Y.), v. 295, p. 2438-2441, doi:10.1126/science.1068797.

Clarke, G.K.C., 2005, SUBGLACIAL PROCESSES: Annual Review of Earth And Planetary Sciences, v. 1, doi:10.1146/annurev.earth.33.092203.122621.

Collins, D.B.G., and Bras, R.L., 2004, Modeling the effects of vegetation-erosion coupling on landscape evolution: JOURNAL OF GEOPHYSICAL RESEARCH, v. 109, p. 1-11, doi:10.1029/2003JF000028.

Conway, H., Hall, B., Denton, G., Gades, A., and Waddington, E., 1999, Past and Future Grounding-Line Retreat of the West Antarctic Ice Sheet: Science, v. 286, doi:10.1126/science.286.5438.280.

Corbett, L.B., Bierman, P.R., and Rood, D.H., 2016, An approach for optimizing in situ cosmogenic 10 Be sample preparation: Quaternary Geochronology, v. 33, p. 24-34, doi:10.1016/j.quageo.2016.02.001.

Denton, G., Bockheim, J., Wilson, C., and Schluchter, C., 1986, LATE CENOZOIC HISTORY OF RENNICK GLACIER AND TALOS DOME, NORTHERN for Quaternary Studies mountain glaciers and the Talos Dome Rennick Glacier. The present-day grounding line is at the glacier midsection near Litell characterize Rocks. Two major - lacia: Gelogical Investigations in Northern Victoria Land: Antarctic Research Series, v. 46, p. 339-375.

Denton, G.H., and Hughes, T.J., 2000, Reconstruction of the Ross Ice Drainage System, Antarctica, at the Last Glacial Maximum ROSS ICE DRAINAGE SYSTEM, ANTARCTICA, AT THE LAST GLACIAL MAXIMUM: Source: Geografiska Annaler. Series A, Physical Geography, v. 823, p. 143-166, http://www.jstor.org/stable/520990. 
Deschamps, P., Durand, N., Bard, E., Hamelin, B., Camoin, G., Thomas, A.L., Henderson, G.M., Okuno, J. 'ichi, and Yokoyama, Y., 2012, Ice-sheet collapse and sea-level rise at the Bølling warming 14,600 years ago: Nature, v. 483, doi:10.1038/nature10902.

Domack, E.W., Jacobson, E.A., Shipp, S.S., and Anderson, J.B., 1999, Late Pleistocene - Holocene retreat of the West Antarctic Ice-Sheet system in: GSA Bulletin, v. 111, p. 1517-1536.

Dorman, L.I., and Valdes, J.F., 1999, Numerical simulation and analytical description of solar neutron transport in the Earth 's atmosphere Numerical simulation and analytical description of solar neutron transport in the Earth ' $s$ atmosphere here neutron attenuation of initial enhances the: Journal of Geophysical Research Atmospheres, doi:10.1029/1999JA900182.

Dunai T., 2010, Cosmogenic Nuclides: Book, p. 187.

Dunne, J., Elmore, D., and Muzikar, P., 1999, Scaling factors for the rates of production of cosmogenic nuclides for geometric shielding and attenuation at depth on sloped surfaces: Geomorphology, doi:10.1016/S0169-555X(98)00086-5.

Dupont, T.K., and Alley, R.B., 2005, Assessment of the importance of ice-shelf buttressing to ice-sheet flow: Geophysical Research Letters, v. 32, doi:10.1029/2004GL022024.

Dupont, T.K., and Alley, R.B., 2006, Role of small ice shelves in sea-level rise: Geophysical Research Letters, v. 33, p. 3-6, doi:10.1029/2005GL025665.

Eaves, S.R., Collins, J.A., Jones, R.S., Norton, K.P., Tims, S.G., and Mackintosh, A.N., 2018, Further constraint of the in situ cosmogenic 10 Be production rate in pyroxene and a viability test for late Quaternary exposure dating: Quaternary Geochronology, v. 48, p. 121-132, doi:10.1016/j.quageo.2018.09.006.

Elliott, C., 2008, Influence of temperature and moisture availability on physical rock weathering along the Victoria Land coast, Antarctica: Antarctic Science, v. 20, p. 61-67, doi:10.1017/\$0954102007000685.

Elmore, D., and Phillips, F.M., 1987, Accelerator mass spectrometry for measurement of long-lived radioisotopes: Science, v. 236, p. 543-550, doi:10.1126/science.236.4801.543.

Fairbanks, R.G., 1989, A 17,000-Year glacio-eustatic sea level record: influence of glacial melting rates on the Younger Dryas event and deep-ocean circulation: Nature, v. 342, p. 637-342.

Favier, L., Durand, G., Cornford, S.L., Gudmundsson, G.H., Gagliardini, O., Gillet-Chaulet, F., Zwinger, T., Payne, A.J., and Le Brocq, A.M., 2014, Retreat of Pine Island Glacier controlled by marine ice-sheet instability: Nature Climate Change, v. 4, p. 117-121, doi:10.1038/nclimate2094.

Feldmann, J., and Levermann, A., 2015, Collapse of the West Antarctic Ice Sheet after local destabilization of the Amundsen Basin: Proceedings of the National Academy of Sciences of the United States of America, v. 112, p. 14191-14196, doi:10.1073/pnas.1512482112.

Felikson, D. et al., 2017, Inland thinning on the Greenland ice sheet controlled by outlet glacier geometry: Nature Geoscience, v. 10, p. 366-369, doi:10.1038/ngeo2934.

Ferrier, K.L., Huppert, K.L., and Perron, J.T., 2013, Climatic control of bedrock river incision: Nature, v. 496, p. 206-209, doi:10.1038/nature11982.

Fink, D., McKelvey, B., Hambrey, M.J., Fabel, D., and Brown, R., 2006, Pleistocene deglaciation chronology of the Amery Oasis and Radok Lake, northern Prince Charles Mountains, Antarctica: Earth and Planetary Science Letters, v. 243, p. 229-243, doi:10.1016/j.epsl.2005.12.006. 
Finocchiaro, F., Baroni, C., Colizza, E., and Ivaldi, R., 2007, Pre-LGM open-water conditions south of the Drygalski Ice Tongue, Ross Sea, Antarctica: Antarctic Science, v. 19, p. 373-377, doi:10.1017/S0954102007000430.

Francisco, A.R.L., 2013, COSMIC RAYS AT EARTH: v. 53, 1-1699 p., doi:10.1017/CBO9781107415324.004.

French, H.M., 2007, the Periglacial Environment: 14-449 p., doi:10.2307/j.ctt1w6tb9v.3.

Friedmann, E.J., Weed, R., and Land, V., 1987, Abiotic Weathering in the Antarctic Cold Desert: Science, v. 236, p. 703-705, doi:10.1126/science.11536571.

Fürst, J.J., Durand, G., Gillet-Chaulet, F., Tavard, L., Rankl, M., Braun, M., and Gagliardini, O., 2016, The safety band of Antarctic ice shelves: Nature Climate Change, v. 6, p. 479-482, doi:10.1038/nclimate2912.

Gagliardini, O., Durand, G., Zwinger, T., Hindmarsh, R.C.A., and Meur, E. Le, 2010, Coupling of ice-shelf melting and buttressing is a key process in ice-sheets dynamics Coupling of ice - shelf melting and buttressing is a key process in ice - sheets dynamics: Geophysical Research Letters, v. 37, doi:10.1029/2010GL043334.

Gaisser, T., 1990, Gamma rays and neutrinos as clues to the origin of high energy cosmic rays: Science, $v$. 247, p. 1049-1056.

Glasser, N., and Bennett, M., 2009, Glacial Geology: Ice Sheets and Landforms, 2nd Edition: 1-377 p.

Glen J., 1952, Experiments on the deformation of ice: Journal of Glaciology, v. 2, p. 111-114.

Goehring, B.M., Balco, G., Todd, C., Moening-Swanson, I., and Nichols, K., 2019, Late-glacial grounding line retreat in the northern Ross Sea, Antarctica: Geology, v. 47, p. 291-294, doi:10.1130/G45413.1.

Goehring, B.M., Schimmelpfennig, I., and Schaefer, J.M., 2014, Capabilities of the lamont-doherty earth observatory in situ 14C extraction laboratory updated: Quaternary Geochronology, v. 19, p. 194-197, doi:10.1016/j.quageo.2013.01.004.

Goethals, M.M., Hetzel, R., Niedermann, S., Wittmann, H., Fenton, C.R., Kubik, P.W., Christl, M., and von Blanckenburg, F., 2009, An improved experimental determination of cosmogenic $10 \mathrm{Be} / 21 \mathrm{Ne}$ and $26 \mathrm{Al} / 21 \mathrm{Ne}$ production ratios in quartz: Earth and Planetary Science Letters, v. 284, p. 187-198, doi:10.1016/j.epsl.2009.04.027.

Golledge, N.R., Kowalewski, D.E., Naish, T.R., Levy, R.H., Fogwill, C.J., and Gasson, E.G.W., 2015, The multimillennial Antarctic commitment to future sea-level rise: Nature, v. 526, p. 421-425, doi:10.1038/nature15706.

Golledge, N.R., Menviel, L., Carter, L., Fogwill, C.J., England, M.H., Cortese, G., and Levy, R.H., 2014, Antarctic contribution to meltwater pulse $1 \mathrm{~A}$ from reduced Southern Ocean overturning: Nature Communications, v. 5, p. 1-10, doi:10.1038/ncomms6107.

Gore, D.B., Creagh, D.C., Burgess, J.S., Colhoun, E.A., Spate, A.P., and Baird, A.S., 1996, Composition, distribution and origin of surficial salts in the Vestfold Hills, East Antarctica: Antarctic Science, v. 8, p. 73-84, doi:10.1017/s0954102096000119.

Gosse, J.C., and Phillips, F.M., 2001, Terrestrial in situ cosmogenic nuclides: Theory and application: Quaternary Science Reviews, doi:10.1016/S0277-3791(00)00171-2.

Greenwood, S.L., Simkins, L.M., Halberstadt, A.R.W., Prothro, L.O., and Anderson, J.B., 2018, Holocene reconfiguration and readvance of the East Antarctic Ice Sheet: Nature Communications, v. 9, doi:10.1038/s41467-018-05625-3. 
Gudmindsson, H., Krug, J., Durand, G., Favier, L., and Galiardini, O., 2012, Northumbria Research Link: The Chryosphere, v. 6, p. 1497-1505, doi:10.5194/tc-6-1497-2012.

Halberstadt, A.R.W., Simkins, L.M., Greenwood, S.L., and Anderson, J.B., 2016, Past ice-sheet behaviour: Retreat scenarios and changing controls in the Ross Sea, Antarctica: Cryosphere, v. 10, p. 1003-1020, doi:10.5194/tc-10-1003-2016.

Hall, B.L., Baroni, C., and Denton, G.H., 2004, Holocene relative sea-level history of the Southern Victoria Land Coast, Antarctica, in Global and Planetary Change, doi:10.1016/j.gloplacha.2003.09.004.

Hall, B.L., and Denton, G.H., 2000a, Extent and chronology of the Ross Sea ice sheet and the Wilson Piedmont Glacier along the Scott Coast at and since the last glacial maximum: Geografiska Annaler, Series A: Physical Geography, v. 82, p. 337-363, doi:10.1111/j.0435-3676.2000.00128.x.

Hall, B.L., and Denton, G.H., 2000b, Radiocarbon chronology of Ross Sea drift, eastern Taylor Valley, Antarctica: Evidence for a grounded ice sheet in the Ross Sea at the last glacial maximum: Geografiska Annaler, Series A: Physical Geography, v. 82, p. 305-336, doi:10.1111/j.0435-3676.2000.00127.x.

Hall, B.L., Denton, G.H., Heath, S.L., Jackson, M.S., and Koffman, T.N.B., 2015, Accumulation and marine forcing of ice dynamics in the western Ross Sea during the last deglaciation: Nature Geoscience, v. 8, p. 625-628, doi:10.1038/ngeo2478.

Hamada, A., 1964, DETERMINATION OF FREE SILICA BY PHOSPHORIC ACID METHOD: Industrial Health, v. 2, p. $181-188$.

Hanebuth, T., Stattegger, K., and Grootes, P.M., 2000, Rapid flooding of the Sunda Shelf: A late-glacial sealevel record: Science, v. 288, p. 1033-1035, doi:10.1126/science.288.5468.1033.

Herbst, K., Kopp, A., and Heber, B., 2013, Influence of the terrestrial magnetic field geometry on the cutoff rigidity of cosmic ray particles: Annales Geophysicae, v. 31, p. 1637-1643, doi:10.5194/angeo-31-16372013.

Hidy, A.J., Gosse, J.C., Blum, M.D., and Gibling, M.R., 2014, Glacial - interglacial variation in denudation rates from interior Texas, USA , established with cosmogenic nuclides: Earth and Planetary Science Letters, v. 390, p. 209-221, doi:10.1016/j.epsl.2014.01.011.

Hilley, G.E., and Porder, S., 2008, A framework for predicting global silicate weathering and CO2 drawdown rates over geologic time-scales: Proceedings of the National Academy of Sciences of the United States of America, v. 105, p. 16855-16859, doi:10.1073/pnas.0801462105.

Hofmann, H.J., Beer, J., Bonani, G., Gunten, H.R. Von, Raman, S., and Bern, C.-, 1987, Beryllium-10: Half-Life and AMS Standards: Special paper from the Swiss National Science Foundationand the U.S. Department of Energy,.

Hunt, A.L., Larsen, J., Bierman, P.R., and Petrucci, G.A., 2008, Investigation of factors that affect the sensitivity of accelerator mass spectrometry for cosmogenic 10Be and 26Al isotope analysis: Analytical Chemistry, v. 80, p. 1656-1663, doi:10.1021/ac701742p.

Ivy-Ochs, S., Schluchter, C., Kubik, P.W., Dittrich-Hannen, B., and Beer, J., 1995, Minimum 10 Be exposure ages of early Pliocene for the Table Mountain plateau and the Sirius Group at Mount Fleming, Dry Valleys, Antarctica: Geology, v. 23, p. 1007-1010, doi:10.1130/00917613(1995)023<1007:MBEAOE>2.3.CO;2. 
Jackson, M.S., Hall, B.L., and Denton, G.H., 2018, Asynchronous behavior of the Antarctic Ice Sheet and local glaciers during and since Termination 1, Salmon Valley, Antarctica: Earth and Planetary Science Letters, v. 482, p. 396-406, doi:10.1016/j.epsl.2017.11.038.

Jacobs, S.S., Jenkins, A., Giulivi, C.F., and Dutrieux, P., 2011, Stronger ocean circulation and increased melting under Pine Island Glacier ice shelf: Nature Geoscience, v. 4, p. 519-523, doi:10.1038/ngeo1188.

Jones, R.S., Mackintosh, A.N., Norton, K.P., Golledge, N.R., Fogwill, C.J., Kubik, P.W., Christl, M., and Greenwood, S.L., 2015, Rapid Holocene thinning of an East Antarctic outlet glacier driven by marine ice sheet instability: Nature Communications, v. 6, doi:10.1038/ncomms9910.

Joughin, I., Smith, B.E., and Medley, B., 2014, Marine Ice Sheet Collapse Potentiall Under Wat for the Thwaites Glacier Basin, West Antarctica: Science, v. 344, p. 735-739.

Kiernan, K., Gore, D.B., Fink, D., White, D.A., McConnell, A., and Sigurdsson, I.A., 2009, Deglaciation and weathering of larsemann hills, East Antarctica: Antarctic Science, v. 21, p. 373-382, doi:10.1017/S0954102009002028.

Kim, S. et al., 2018, Seismic stratigraphy of the Central Basin in northwestern Ross Sea slope and rise, Antarctica: Clues to the late Cenozoic ice-sheet dynamics and bottom-current activity: Marine Geology, v. 395, p. 363-379, doi:10.1016/j.margeo.2017.10.013.

Kingslake, J., Scherer, R.P., Albrecht, T., Coenen, J., Powell, R.D., Reese, R., Stansell, N.D., Tulaczyk, S., Wearing, M.G., and Whitehouse, P.L., 2018, Extensive retreat and re-advance of the West Antarctic Ice Sheet during the Holocene: Nature, v. 558, p. 430-434, doi:10.1038/s41586-018-0208-x.

Kohl, C.P., and Nishiizumi, K., 1992, Chemical isolation of quartz for measurement of in-situ -produced cosmogenic nuclides: Geochimica et Cosmochimica Acta, doi:10.1016/0016-7037(92)90401-4.

Kohman, T.P., 1997, Aluminum-26: A nuclide for all seasons: Journal of Radioanalytical and Nuclear Chemistry, v. 219, p. 165-176.

Kolck, U. Van et al., 2002, Short-Lived Nuclides in Hibonite Grains from Murchison : Evidence for Solar System Evolution: Science, v. 298, p. 2182-2186.

Korschinek, G. et al., 2010, A new value for the half-life of 10Be by Heavy-lon Elastic Recoil Detection and liquid scintillation counting: Nuclear Instruments and Methods in Physics Research, Section B: Beam Interactions with Materials and Atoms, v. 268, p. 187-191, doi:10.1016/j.nimb.2009.09.020.

Korte, M., Constable, C., Donadini, F., and Holme, R., 2011, Reconstructing the Holocene geomagnetic field: Earth and Planetary Science Letters, v. 312, p. 497-505, doi:10.1016/j.epsl.2011.10.031.

Lal, D., 1991, Cosmic ray labeling of erosion surfaces: in situ nuclide production rates and erosion models: Earth and Planetary Science Letters, doi:10.1016/0012-821X(91)90220-C.

Lee, J. II, McKay, R.M., Golledge, N.R., Yoon, H. II, Yoo, K.C., Kim, H.J., and Hong, J.K., 2017, Widespread persistence of expanded East Antarctic glaciers in the southwest Ross Sea during the last deglaciation: Geology, v. 45, p. 403-406, doi:10.1130/G38715.1.

Lifton, N., Sato, T., and Dunai, T.J., 2014, Scaling in situ cosmogenic nuclide production rates using analytical approximations to atmospheric cosmic-ray fluxes: Earth and Planetary Science Letters, v. 386, p. 149160, doi:10.1016/j.epsl.2013.10.052.

Liu, J., Milne, G.A., Kopp, R.E., Clark, P.U., and Shennan, I., 2015, Sea-level constraints on the amplitude and source distribution of Meltwater Pulse 1A: Nature Geoscience, v. 9, p. 6-12, doi:10.1038/ngeo2616. 
Lowry, D.P., Golledge, N.R., Bertler, N.A.N., Jones, R.S., and McKay, R., 2019, Deglacial grounding-line retreat in the Ross Embayment, Antarctica, controlled by ocean and atmosphere forcing: Science Advances, v. 5, p. eaav8754, doi:10.1126/sciadv.aav8754.

Macayeal, D.R., and Sergienko, O. V, 2013, The flexural dynamics of melting ice shelves: Annals of Glaciology, v. 54, p. 1-10, doi:10.3189/2013AoG63A256.

Mackintosh, A.N. et al., 2014, Retreat history of the East Antarctic Ice Sheet since the Last Glacial Maximum: Quaternary Science Reviews, doi:10.1016/j.quascirev.2013.07.024.

Mackintosh, A. et al., 2011, Retreat of the East Antarctic ice sheet during the last glacial termination: Nature Geoscience, v. 4, p. 195-202, doi:10.1038/ngeo1061.

Mackintosh, A., White, D., Fink, D., Gore, D.B., Pickard, J., and Fanning, P.C., 2007, Exposure ages from mountain dipsticsk in Mac. Robertson Land, East Antarctica, indicate little change in ice-sheet thickness since the Last Glacial Maximum: Geology, v. 35, p. 551-554, doi:10.1130/G23503A.1.

Mariotti, A., Blard, P., Charreau, J., and Petit, C., 2019, Denudation systematics inferred from in situ cosmogenic 10 Be concentrations in fine $(50-100 \mu \mathrm{m})$ and medium ( $100-250 \mu \mathrm{m})$ sediments of the Var River basin, southern French Alps: Earth Surface Dynamics, p. 1-24.

Marrero, S.M., Hein, A.S., Naylor, M., Attal, M., Shanks, R., Winter, K., Woodward, J., Dunning, S., Westoby, M., and Sugden, D., 2018, Controls on subaerial erosion rates in Antarctica: Earth and Planetary Science Letters, v. 501, p. 56-66, doi:10.1016/j.epsl.2018.08.018.

Marshall, J.A., Roering, J.J., Bartlein, P.J., Gavin, D.G., Granger, D.E., Rempel, A.W., Praskievicz, S.J., and Hales, T.C., 2015, Frost for the trees : Did climate increase erosion in unglaciated landscapes during the late Pleistocene ? Science Advances,.

Mayewski, P.A., 1975, Glacial Geologic Investigation of Upper Rennick Glacier Region , Northern Victoria Land: Antarctic Journal, v. 7, p. 164-166.

Mayewski, P.A., Attig, J.W., and Drewry, D.J., 1979, PATTERN OF ICE SURFACE LOWERING FOR: Journal of Glaciology, v. 22.

McKay, R.M., Dunbar, G.B., Naish, T.R., Barrett, P.J., Carter, L., and Harper, M., 2008, Retreat history of the Ross Ice Sheet (Shelf) since the Last Glacial Maximum from deep-basin sediment cores around Ross Island: Palaeogeography, Palaeoclimatology, Palaeoecology, v. 260, p. 245-261, doi:10.1016/j.palaeo.2007.08.015.

McKay, R., Golledge, N.R., Maas, S., Naish, T., Levy, R., Dunbar, G., and Kuhn, G., 2016, Antarctic marine icesheet retreat in the Ross Sea during the early Holocene: Geology, v. 44, p. 7-10, doi:10.1130/G37315.1.

McKibben, R.B., Pyle, K.R., and Simpson, J.A., 1982, The Galactic Cosmic-ray Radial Intensity Gradient and Large-scale Modulation in the Heliosphere: The Astrophysical Journal, v. 254, p. L23-L27.

Meneghel, M., Bondesan, A., Salvatore, M., and Orombelli, G., 1999, A model of the glacial retreat of Upper Rennick Glacier, Victoria Land , A Illodel of the glacial retreat of upper Rennick Glacier ,: Annals of Glaciology, v. 29, doi:10.3189/172756499781821463.

Mengel, M., and Levermann, A., 2014, Ice plug prevents irreversible discharge from east Antarctica: Nature Climate Change, v. 4, p. 451-455, doi:10.1038/nclimate2226.

Le Meur, E., Gagliardini, O., Zwinger, T., and Ruokolainen, J., 2004, Glacier flow modelling: A comparison of the Shallow Ice Approximation and the full-Stokes solution: Comptes Rendus Physique, v. 5, p. 709722, doi:10.1016/j.crhy.2004.10.001. 
Middleton, J.L., Ackert, R.P., and Mukhopadhyay, S., 2012, Pothole and channel system formation in the McMurdo Dry Valleys of Antarctica: New insights from cosmogenic nuclides: Earth and Planetary Science Letters, v. 355-356, p. 341-350, doi:10.1016/j.epsl.2012.08.017.

Mifsud, C., Fujioka, T., and Fink, D., 2013, Extraction and purification of quartz in rock using hot phosphoric acid for in situ cosmogenic exposure dating: Nuclear Instruments and Methods in Physics Research, Section B: Beam Interactions with Materials and Atoms, v. 294, p. 203-207, doi:10.1016/j.nimb.2012.08.037.

Milliman, J.D., and Syvitski, J.P.M., 1992, Geomorphic / Tectonic Control of Sediment Discharge to the Ocean : The Importance of Small Mountainous Rivers: Journal of Geology, v. 100, p. 525-544.

Monaghan, M., Krishnaswami, S., and Turekian, K., 1985, The global-average production rate of 10 Be: Earth and Planetary Science Letters, v. 76, p. 279-287.

Montgomery, D.R., 2007, Soil erosion and agricultural sustainability: PNAS, v. 104, p. 13268-13272.

Morris, M., Armstrong, A., and Thomas, A., 1979, DIRECT MEASUREMENT OF STRESS AT THE BASE OF A GLACIER Wherev er it has been possibl e to observe the sole of a temper contain above immed tres of rock debris in the several centime and particle ual Individ sole is an import ant erosive agent .: Journal fo Glaciology, v. 22.

N.P.M. Van lipzig, J. Turner, S.A. Colwell, and M.R.V.D.B., 2004, SHORT COMMUNICATION THE NEARSURFACE WIND FIELD OVER THE ANTARCTIC CONTINENT: International Journal of Climatology, v. 1982, p. 1973-1982, doi:10.1002/joc.1090.

Naish, T., Powell, R., Levy, R., Henrys, S., Krissek, L., Niessen, F., Pompilio, M., Scherer, R., and Wilson, G.S., 2007, Synthesis of the initial scientific results of the MIS Project (AND-1B core), Victoria Land Basin, Antarctica: Terra Antartica, v. 14, p. 317-327.

Nichols, K.A., and Goehring, B.M., 2019, Isolation of quartz for cosmogenic in situ \&lt;sup\&gt;14\&lt;/sup\&gt;C analysis: Geochronology Discussions, p. 1-14, doi:10.5194/gchron-20197.

Di Nicola, L., Baroni, C., Strasky, S., Salvatore, M.C., Schlüchter, C., Akçar, N., Kubik, P.W., and Wieler, R., 2012, Multiple cosmogenic nuclides document the stability of the East Antarctic Ice Sheet in northern Victoria Land since the Late Miocene (5-7 Ma): Quaternary Science Reviews, v. 57, p. 85-94, doi:10.1016/j.quascirev.2012.09.026.

Nishiizumi, K., 1989, Cosmic ray production rates of 10Be and 26Al in quartz from glacially polished rocks: Journal of Geophysical Research, v. 94, doi:10.1029/jb094ib12p17907.

Nishiizumi, K., 2004, Preparation of Al AMS standards: Nuclear Instrument and methods in Physics Research, v. 224, p. 388-392, doi:10.1016/j.nimb.2004.04.075.

Nishiizumi, K., Caffe, M.W., and Depaolo, D.J., 2000, Preparation of Ca AMS standards: Nuclear Instrument and methods in Physics Research B, v. 172, p. 399-403.

Nishiizumi, K., Imamura, M., Caffee, M.W., Southon, J.R., Finkel, R.C., and McAninch, J., 2007, Absolute calibration of 10Be AMS standards: Nuclear Instruments and Methods in Physics Research, Section B: Beam Interactions with Materials and Atoms, doi:10.1016/j.nimb.2007.01.297.

Nishiizumi, K., Kohl, C., Arnold, J., Klein, J., and Middelton, R., 1991, Cosmic ray produced 10Be and 26Al in Antarctic rocks: exposure and erosion history: Earth and Planetary Science Letters, v. 104, p. 440-454. 
Nishiizumi, K., Lalt, D., Klein, J., Middleton, R., and Arnold, J.R., 1986, Production of 10 Be and 26 Al by cosmic rays in terrestrial quartz: Nature, v. 319, p. 33-35.

Norton, K.P., von Blanckenburg, F., Schlunegger, F., Schwab, M., and Kubik, P.W., 2008, Cosmogenic nuclidebased investigation of spatial erosion and hillslope channel coupling in the transient foreland of the Swiss Alps: Geomorphology, doi:10.1016/j.geomorph.2007.07.013.

Nye J., 1952, The mechanics of glacier flow: Journal of Glaciology, v. 2, p. 82-93.

Oberholzer, P., Baroni, C., Salvatore, M.C., Baur, H., and Wieler, R., 2008, Dating late Cenozoic erosional surfaces in Victoria Land, Antarctica, with cosmogenic neon in pyroxenes: Antarctic Science, v. 20, p. 89-98, doi:10.1017/S095410200700079X.

Oberholzer, P., Baroni, C., Schaefer, J.M., Orombelli, G., Ochs, S.I., Kubik, P.W., Baur, H., and Wieler, R., 2003, Limited Pliocene/Pleistocene glaciation in Deep Freeze Range, northern Victoria Land, Antarctica, derived from in situ cosmogenic nuclides: Antarctic Science, v. 15, p. 493-502, doi:10.1017/S0954102003001603.

Ochs, M., and Ivy-Ochs, S., 1997, The chemical behavior of $\mathrm{Be}, \mathrm{Al}, \mathrm{Fe}, \mathrm{Ca}$ and $\mathrm{Mg}$ during AMS target preparation from terrestrial silicates modeled with chemical speciation calculations: Nuclear Instruments and Methods in Physics Research Section B: Beam Interactions with Materials and Atoms, v. 123 , p. 235-240, doi:10.1016/S0168-583X(96)00680-5.

Orman, J.A. Van, and Krawczynski, M.J., 2015, Theoretical constraints on the isotope effect for diffusion in minerals: Geochemica et Cosmochimica, v. 164, p. 365-381.

Parker, R.L., 2017, Ross Sea Deglaciation and Holocene Paleoenvironments: Masters Thesis: Otago University, p. 161.

Pattyn, F., 2003, A new three-dimensional higher-order thermomechanical ice sheet model : Basic sensitivity , ice stream development , and ice flow across subglacial lakes: Journal of Geophysical Letters, v. 108, p. 1-15, doi:10.1029/2002JB002329.

Payne, A.J., Vieli, A., Shepherd, A.P., Wingham, D.J., and Rignot, E., 2004, Recent dramatic thinning of largest West Antarctic ice stream triggered by oceans: Geophysical Research Letters, v. 31, p. 1-4, doi:10.1029/2004GL021284.

Perotti, M., Zurli, L., Sandroni, S., Cornamusini, G., and Talarico, F., 2018, Provenance of Ross Sea Drift in McMurdo Sound ( Antarctica ) and implications for middle-Quaternary to LGM glacial transport : New evidence from petrographic data: Sedimentary Geology, v. 371, p. 41-54, doi:10.1016/j.sedgeo.2018.04.009.

Pilchowski, J., Kopp, A., Herbst, K., and Heber, B., 2010, On the definition and calculation of a generalised Mcllwain parameter: Astrophysics and Space Sciences Transactions, v. 6, p. 9-17, doi:10.5194/astra6-9-2010.

Pollard, D., Holland, D., and Gomez, N., 2015, Antarctic Ice-Sheet mass loss: Nature Communications, v. 6, p. 1-8, doi:10.1038/ncomms9798.

Poluianov, S. V, Kovaltsov, G.A., Mishev, A.L., and Usoskin, I.G., 2016, Production of cosmogenic isotopes $7 \mathrm{Be}, 10 \mathrm{Be}, 14 \mathrm{C}, 22 \mathrm{Na}$, and $36 \mathrm{Cl}$ in the atmosphere: Altitudinal profiles: Journal of Geophysical research: Atmospheres, v. June, p. 8125-8136, doi:10.1002/2016JD025034.New. 
Portenga, E.W., Bierman, P.R., Duncan, C., Corbett, L.B., Kehrwald, N.M., and Rood, D.H., 2015, Erosion rates of the Bhutanese Himalaya determined using in situ-produced 10Be: Geomorphology, v. 233, p. 112126, doi:10.1016/j.geomorph.2014.09.027.

Portenga, E.W., Bierman, P.R., and Resources, N., 2011, Understanding Earth' s eroding surface with 10 Be: GSA Today, v. 21, p. 4-10, doi:10.1130/G111A.1.

Pörtner, H.-O. et al., 2019, The Ocean and Cryosphere in a Changing Climate: Summary for Policymakers. In: IPCC Special Report on the Ocean and Cryosphere in a Changing Climate,.

Radokl, U., Allison, l., and Wendler8, G., 1996, Atmospheric surface pressure over the interior of Antarctica: Antarctic Science, v. 8, p. 209-21, http://journals.cambridge.org.

Reese, R., Gudmundsson, G.H., Levermann, A., and Winkelmann, R., 2018, The far reach of ice-shelf thinning in Antarctica: Nature Climate Change, v. 8, p. 53-57, doi:10.1038/s41558-017-0020-x.

Rich, H., Monash, E., Iverson, N.R., Hanson, B., Hooke, R.L., and Jansson, P., 1990, Flow Mechanism of Glaciers on Soft Beds: Science, v. 267, p. 80-82.

Rightmire, R., Kohman, T., and Allen, A., 1957, Production of Carrier-free Aluminum- and Sodium- *: International Journal of Applied Radiation and Isotopes, v. 2, p. 274-279.

Rignot, E., Jacobs, S., Mouginot, J., and Scheuchl, B., 2013, Ice-Shelf Melting Around Antarctica: Science, v. 341, p. 1334-1337.

Rignot, E., Mouginot, J., Morlighem, M., Seroussi, H., and Scheuchl, B., 2014, Widespread , rapid grounding line retreat of Pine Island, Thwaites, Smith, and Kohler glaciers, West Antarctica ,: Geophysical Reasearch Letters, p. 3502-3509, doi:10.1002/2014GL060140.Received.

Ritz, C., Edwards, T.L., Durand, G., Payne, A.J., Peyaud, V., and Hindmarsh, R.C.A., 2015, Potential sea-level rise from Antarctic ice-sheet instability constrained by observations: Nature, v. 528, p. 115-118, doi:10.1038/nature16147.

Sanz, J., and Buchlin, R.J., 2012, Evaluation of the Antarctic surface wind climate from ERA reanalyses and RACMO2 / ANT simulations based on automatic weather stations Evaluation of the antarctic surface wind climate from ERA reanalyses and RACMO2 / ANT simulations based on automatic weat: Climat Dynamics, doi:10.1007/s00382-012-1396-y.

Scambos, T.A., Bohlander, J.A., Shuman, C.A., and Skvarca, P., 2004, Glacier acceleration and thinning after ice shelf collapse in the Larsen B embayment, Antarctica: Geophysical Research Letters, doi:10.1029/2004GL020670.

Schafer, J.M., Ivy-Ochs, S., Wieler, R., Leya, I., Baur, H., Denton, G.H., and Schluchter, C., 1999, Cosmogenic noble studies in the oldest landscape on earth: Surface exposure ages of the Dry Valleys, Antarctica: Earth and Planetary Science Letters, v. 167, p. 215-226, doi:10.1016/S0012-821X(99)00029-1.

Schildgen, T.F., Phillips, W.M., and Purves, R.S., 2005, Simulation of snow shielding corrections for cosmogenic nuclide surface exposure studies: Geomorphology, doi:10.1016/j.geomorph.2004.05.003.

Schoof, C., 2007a, Ice sheet grounding line dynamics: Steady states, stability, and hysteresis: Journal of Geophysical Research: Earth Surface, doi:10.1029/2006JF000664.

Schoof, C., 2007b, Marine ice-sheet dynamics. Part 1. The case of rapid sliding: Journal of Fluid Mechanics, v. 573, p. 27-55, doi:10.1017/\$0022112006003570. 
Schumm, W.B.L. and S.A., 1958, Yield of Sediment in Relation to Mean Annual Precipitation: American Geophysical Union, v. 39.

Shea, M.A., Smart, D.F., and Gentile, L.C., 1987, Estimating cosmic ray vertical cutoff rigidities as a function of the Mcllwain L-parameter for different epochs of the geomagnetic field: Physics of the Earth and Planetary Interiors, doi:10.1016/0031-9201(87)90145-2.

Shipp, S.S., Anderson, J.B., and Domack, E.W., 1999, The Ross Sea : Part 1 - Geophysical results: v. 111, 1486-1516 p., doi:10.1130/0016-7606(1999)111<1486.

Shipp, S.S., Wellner, J.S., and Anderson, J.B., 2019, Retreat signature of a polar ice stream : sub-glacial geomorphic features and sediments from the Ross Sea, Antarctica Downloaded from http://sp.lyellcollection.org/ at Victoria University of Wellington: In Glacier-Influenced Sediment on High-Latitude Continental Margins, p. 277-304.

Shuster, D., and Farley, K., 2005, Diffusion kinetics of proton-induced 21Ne, $3 \mathrm{He}$, and $4 \mathrm{He}$ in quartz: Geochemica et Cosmichimica, v. 69, p. 2349-2359, doi:10.1016/j.gca.2004.11.002.

Skov, D.S., Egholm, D.L., Jansen, J.D., Sandiford, M., and Knudsen, M.F., 2019, Detecting landscape transience with in situ cosmogenic $14 \mathrm{C}$ and 10Be: Quaternary Geochronology, v. 54, p. 101008, doi:10.1016/j.quageo.2019.101008.

Smart, D.F., and Shea, M.A., 2009, Fifty years of progress in geomagnetic cutoff rigidity determinations: Advances in Space Research, doi:10.1016/j.asr.2009.07.005.

Smart, D.F., and Shea, M.A., 1985, GALACTIC COSMIC RADIATION AND SOLAR ENERGETIC PARTICLES 6.1 NOMENCLATURE AND DEFINITIONS: Handbook of geophysics and the space environment, p. 6.1-6.29.

Spector, P., Stone, J., Cowdery, S.G., Hall, B., Conway, H., and Bromley, G., 2017, Rapid early-Holocene deglaciation in the Ross Sea, Antarctica: Geophysical Research Letters, v. 44, p. 7817-7825, doi:10.1002/2017GL074216.

Stewart, C.L., Christoffersen, P., Nicholls, K.W., Williams, M.J.M., and Dowdeswell, J.A., 2019, Basal melting of Ross Ice Shelf from solar heat absorption in an ice-front polynya: Nature Geoscience, v. 12, p. 435440, doi:10.1038/s41561-019-0356-0.

Stone, J.O., 2000, Air pressure and cosmogenic isotope production: JOURNAL OF GEOPHYSICAL RESEARCH, v. 105759, p. 753-23, doi:10.1029/2000JB900181.

Stone, J.O., Balco, G.A., Sugden, D.E., Caffee, M.W., Sass, L.C., Cowdery, S.G., and Siddoway, C., 2003, Holocene deglaciation of Marie Byrd Land, West Antarctica: Science, v. 299, p. 99-102, doi:10.1126/science.1077998.

Sugden, D.E., Balco, G., Cowdery, S.G., Stone, J.O., and Sass, L.C., 2005, Selective glacial erosion and weathering zones in the coastal mountains of Marie Byrd Land, Antarctica: Geomorphology, doi:10.1016/j.geomorph.2004.10.007.

Summerfield, M.A., Stuart, F.M., Cockburn, H.A.P., Sugden, D.E., Denton, G.H., Dunai, T., and Marchant, D.R., 1999, Long-term rates of denudation in the Dry Valleys, Transantarctic Mountains, southern Victoria Land, Antarctica based on in-situ-produced cosmogenic 21Ne: Geomorphology, v. 27, p. 113-129, doi:10.1016/S0169-555X(98)00093-2.

Syvitski, J.P.M., 2003, Supply and flux of sediment along hydrological pathways : research for the 21st century: Global and Planetary Cange, v. 39, p. 1-11, doi:10.1016/S0921-8181(03)00008-0.

Thomas, J.H., Rau, R.L., and Skelton, R.T., 1984, Half-life fo 26Al: Physical Review C, v. 30, p. 385-387. 
Todd, C., Stone, J., Conway, H., Hall, B., and Bromley, G., 2010, Late Quaternary evolution of Reedy Glacier, Antarctica: Quaternary Science Reviews, doi:10.1016/j.quascirev.2010.02.001.

Tremblay, M.M., Shuster, D.L., and Balco, G., 2014, ScienceDirect Diffusion kinetics of $3 \mathrm{He}$ and $21 \mathrm{Ne}$ in quartz and implications for cosmogenic noble gas paleothermometry: GEOCHIMICA ET COSMOCHIMICA ACTA, v. 142, p. 186-204, doi:10.1016/j.gca.2014.08.010.

Trull, T., Brown, E., Marty, B., Raisbeck, M., and Yiou, F., 1995, Cosmogenic 10Be and 3 he accumulation in Pleistocene beach terraces in Death Valley, California, U.S.A.: Implications for cosmic-ray exposure dating of younge surfaces in hot climates: Chemical Geology, v. 119, p. 191-207.

Van Der Wateren, F.M., Dunai, T.J., Van Balen, R.T., Klas, W., Verbers, A.L.L.M., Passchier, S., and Herpers, U., 1999, Contrasting neogene denudation histories of different structural regions in the transantarctic mountains rift flank constrained by cosmogenic isotope measurements: Global and Planetary Change, v. 23, p. 145-172, doi:10.1016/\$0921-8181(99)00055-7.

Waters R., 1957, Differential Weathering and Erosion on Oldlands: The Geographical Journal, v. 123, p. 503509.

Weber, M.E. et al., 2014, Millennial-scale variability in Antarctic ice-sheet discharge during the last deglaciation: Nature, v. 510, p. 134-138, doi:10.1038/nature13397.

Webster, J.M., Clague, D.A., Riker-Coleman, K., Gallup, C., Braga, J.C., Potts, D., Moore, J.G., Winterer, E.L., and Paull, C.K., 2004, Drowning of the $-150 \mathrm{~m}$ reef off Hawaii: A casualty of global meltwater pulse $1 \mathrm{~A}$ ? Geology, v. 32, p. 249-252, doi:10.1130/G20170.1.

Weertman, J., 1974, STABILITY OF THE JUNCTION OF AN ICE SHEET AND AN ICE SHELF: Journal of GlacioJoD, v. 13.

Welten, K.C., Folco, L., Nishiizumi, K., Caffee, M.W., Grimberg, A., Meier, M.M.M., and Kober, F., 2008, Meteoritic and bedrock constraints on the glacial history of Frontier Mountain in northern Victoria Land, Antarctica: Earth and Planetary Science Letters, v. 270, p. 308-315, doi:10.1016/j.epsl.2008.03.052.

White, D.A., Bennike, O., Berg, S., Harley, S.L., Fink, D., Kiernan, K., McConnell, A., and Wagner, B., 2009, Geomorphology and glacial history of Rauer Group, East Antarctica: Quaternary Research, v. 72, p. 8090, doi:10.1016/j.yqres.2009.04.001.

Wittmann, H., Blanckenburg, F. Von, Kruesmann, T., Norton, K.P., and Kubik, P.W., 2007, Relation between rock uplift and denudation from cosmogenic nuclides in river sediment in the Central Alps of Switzerland: Journal of Geophysical Research, v. 112, p. 1-20, doi:10.1029/2006JF000729.

Zerathe, S., Blard, P.H., Braucher, R., Bourlès, D., Zerathe, S., Audin, L., Carcaillet, J., Delgado, F., and Benavente, C., 2017, Toward the feldspar alternative for cosmogenic 10Be applications: Quaternary Geochronology, v. 41, p. 83-96, doi:10.1016/j.quageo.2017.06.004. 